\title{
Maternal hemodynamic and volume adaptation to pregnancy : an observational study in normal pregnancies and pregnancies complicated by fetal growth retardation
}

Citation for published version (APA):

Duvekot, J. J. (1994). Maternal hemodynamic and volume adaptation to pregnancy : an observational study in normal pregnancies and pregnancies complicated by fetal growth retardation. [Doctoral Thesis, Maastricht University]. Rijksuniversiteit Limburg. https://doi.org/10.26481/dis.19941202jd

Document status and date:

Published: 01/01/1994

DOI:

10.26481/dis.19941202jd

Document Version:

Publisher's PDF, also known as Version of record

Please check the document version of this publication:

- A submitted manuscript is the version of the article upon submission and before peer-review. There can be important differences between the submitted version and the official published version of record. People interested in the research are advised to contact the author for the final version of the publication, or visit the DOI to the publisher's website.

- The final author version and the galley proof are versions of the publication after peer review.

- The final published version features the final layout of the paper including the volume, issue and page numbers.

Link to publication

\footnotetext{
General rights rights.

- You may freely distribute the URL identifying the publication in the public portal. please follow below link for the End User Agreement:

www.umlib.nl/taverne-license

Take down policy

If you believe that this document breaches copyright please contact us at:

repository@maastrichtuniversity.nl

providing details and we will investigate your claim.
}

Copyright and moral rights for the publications made accessible in the public portal are retained by the authors and/or other copyright owners and it is a condition of accessing publications that users recognise and abide by the legal requirements associated with these

- Users may download and print one copy of any publication from the public portal for the purpose of private study or research.

- You may not further distribute the material or use it for any profit-making activity or commercial gain

If the publication is distributed under the terms of Article 25fa of the Dutch Copyright Act, indicated by the "Taverne" license above, 


\section{MATERNAL HEMODYNAMIC AND VOLUME ADAPTATION TO PREGNANCY}

AN OBSERVATIONAL STUDY IN NORMAL PREGNANCIES AND PREGNANCIES COMPLICATED BY FETAL GROWTH RETARDATION 
(C) J.J. Duvekot, Rotterdam 1994

\section{CIP-DATA KONINKLIJKE BIBLIOTHEEK, DEN HAAG}

Duvekot, Johannes Jürg

Maternal hemodynamic and volume adaptation to pregnancy: an observational study in normal pregnancies and pregnancies complicated by fetal growth retardation / Johannes Jürg Duvekot. -Maastricht: Universitaire Pers Maastricht, Ill. Thesis Maastricht. - With ref. - With summary in Dutch and Bärndütsch. ISBN 90-5278-167-2

Subject headings: pregnancy / hemodynamics / volume homeostasis

Illustratie omslag: G. van Rooy

Typografie: G. van Rooy

RL Design, Rijksuniversiteit Limburg | Maastricht

Druk: Datawyse | Universitaire Pers Maastricht 


\section{MATERNAL HEMODYNAMIC AND VOLUME ADAPTATION TO PREGNANCY}

\section{AN OBSERVATIONAL STUDY IN NORMAL PREGNANCIES AND PREGNANCIES COMPLICATED BY FETAL GROWTH RETARDATION}

\section{PROEFSCHRIFT}

ter verkrijging van de graad van doctor aan de Rijksuniversiteit Limburg te Maastricht, op gezag van de Rector Magnificus, Prof.dr. H. Philipsen, volgens het besluit van het College van Dekanen, in het openbaar te verdedigen op vrijdag 2 december 1994 om 14.00 uur

door

Johannes Jürg Duvekot 
Promotor:

Co-promotor:

Beoordelingscommissie: Prof.dr. H.A.J. Struijker Boudier

(voorzitter)

Prof.dr. J.M. Davison

(University of Newcastle, Great Britain)

Prof.dr. P.W. de Leeuw

Prof.dr. R.S. Reneman

Prof.dr. H.J.J. Wellens

The studies described in this thesis were financially supported by Schering Nederland BV, Weesp, The Netherlands.

Financial support by the Netherlands Heart Foundation and the Dutch Kidney Foundation for the publication of this thesis is gratefully acknowledged. 
"There is no other purpose than to live, and to live is to relate.

In isolation there may be survival, but only in relationship is there life.

Medicine is part of living, not vice versa."

Shem S. The House of God. 3rd ed. New York:

The Bantam Doubleday Dell Publishing Group, 1988.

The most melancholy of human reflections,

perbaps, is that, on the whole,

it is a question whether the benevolence of mankind does most good or harm.

Bagehot W. Physics and Politics, No. V, 1864.

ter nagedachtenis aan mijn moeder

aan Hélène, Martijne en Carlijn 



\section{CONTENTS}

Abbreviations

page

Chapter 1

Introduction.

Chapter 2

Maternal cardiovascular hemodynamic adaptation

to pregnancy.

Chapter 3

Renal hemodynamics and volume homeostasis in pregnancy.

Chapter 4

Measurement of anterior-posterior diameter of inferior vena cava by ultrasonography: a new non-invasive method to assess acute changes in vascular filling state.

Chapter 5

Volume-dependent echocardiographic parameters are unsuitable for estimating baseline blood volume but are useful for detecting acute changes in vascular filling state.

Chapter 6

Early-pregnancy changes in hemodynamics and volume homeostasis are consecutive adjustments triggered by a primary fall in systemic vascular tone.

Chapter 7 Severely impaired fetal growth is preceded by maternal hemodynamic maladaptation in very early pregnancy.

Chapter 8

Maternal volume homeostasis in early pregnancy in relation to fetal growth retardation.

Chapter 9

General discussion.

Summary.

Dankwoord

Publications 
cansan 


\section{ABBREVIATIONS}

ACTH adrenocorticotropic hormone

ANP atrial natriuretic peptide

FSE fractional salt excretion

GFR glomerular filtration rate

hCG human chorionic gonadotropin

hPL human placental lactogen

IUGR intra-uterine growth retardation

IVCD inferior vena cava diameter

IVST interventricular septal thickness

LAdiam left atrial diameter

LVEDD left ventricular end-diastolic dimension

LVESD left ventricular end-systolic dimension

LVET left ventricular ejection time

MAP mean arterial pressure

PRC plasma renin concentration

PWT posterior left ventricular wall thickness

SD standard deviation

SEM standard error of the mean

SGA small for gestational age

TPVR total peripheral vascular resistance

Vcf circumferential fibre shortening 
Chapter 1

INTRODUCTION 


\subsection{Introduction}

Maternal adaptation to human pregnancy consists, among other things, of profound changes in hemodynamics, renal function and volume homeostasis. This is illustrated by increases of approximately $50 \%$ in cardiac output, glomerular filtration rate and plasma volume, and by a decrease of approximately $20 \%$ in mean arterial pressure. Most hemodynamic and renal adjustments are already fully expressed by the end of the first trimester, with the exception of the plasma volume, which increases gradually from early pregnancy until the beginning of the 3rd trimester. Most of our current knowledge about these adjustments has been extrapolated from the difference between the midpregnant and nonpregnant states. Consequently, both the underlying mechanism and the functional meaning of the early-pregnancy changes are poorly understood. For instance, cardiac output increases without concomitant increase in $\mathrm{O}_{2}$ uptake, supporting the idea of a development of systemic hyperperfusion with compensatory arteriovenous shunting. Similarly, it is still not settled why and how the glomerular filtration rate and effective renal plasma flow increase by more than $50 \%$ in early pregnancy, a rise unmatched in any other clinical and experimental condition. The systemic and renal adjustments could have developed in response to a primary state of hypervolemia. However, the concomitant endocrine profile is consistent with active volume retention triggered by a state of vascular underfill. So far, no satisfactory explanation has been given for this discrepancy.

In pregnancies complicated by fetal growth retardation or preeclampsia, the normal rise in cardiac output, glomerular filtration rate and plasma volume is either reduced or absent. Even in midpregnancy, well before the development of manifest symptomatology, these subjects are found to differ from subjects with uneventful pregnancies. At that time they display a defective plasma volume and cardiac output rise, while the fall in arterial pressure and loss of vascular inertia to angiotensin II are absent. It is still not settled whether these midtrimester abnormalities are primary or reflect ongoing maladaptation from very early pregnancy on.

It may be puzzling for the uninformed reader why this obviously important period of early pregnancy is still largely unexplored. The following factors have obstructed the study of the early-pregnancy adaptation. Until a few years ago, the first trimester was considered trivial with respect to actual metabolic and hemodynamic demands by the conceptus and, therefore, less interesting for study purposes. Serial measurement of cardiac output, plasma volume and renal function during embryogenesis, by means of classical dilution techniques using dye or radioactivity, was controversial and potential harmful. The classical dilution techniques are associated with a large measurement error. In addition, they are invasive and thus cumbersome to apply serially. Potential candidates should enter the study in a very early stage of pregnancy.

This aspect, together with the implications outlined above, complicates the recruitment of an adequate number of subjects. The only suitable animal model that could serve as a usable reference for human pregnancy is the primate. The obvious limitations to studies in this animal emphasize the need to study the early maternal adaptations in the pregnant woman, using noninvasive methodology. 


\subsection{Aims of the study}

The present study was divided into three major parts:

1. The first part consisted of two validation studies in nonpregnant healthy volunteers to assess whether ultrasonic cardiovascular dimensions and their changes induced by volume loading provide usable information about concomitant blood volume, vascular filling state and the magnitude of the changes induced. If proved suitable, serial application of these new methods in early pregnancy was expected to provide valuable noninvasive information about absolute blood volume, vascular filling state and the pattern of change in these parameters in the first trimester.

2. The second part consisted of an observational study in early-pregnant subjects with uneventful pregnancies. Possible interdependence between the various early-pregnancy adjustments was determined by serial measurement of a large number of hemodynamic, renal and volume parameters, together with peripheral steroid levels in the first trimester of pregnancy.

3. The third part consisted of a similar observational study as described above, in subjects with pregnancies complicated by fetal growth retardation. It is known from epidemiological studies that the recruitment of pregnant subjects from a subfertility clinic is associated with a high rate of spontaneous abortion and fetal growth retardation. In fact, 6 of the 24 subjects enrolled in the physiologic study developed fetal growth retardation in the second half of pregnancy. The earlypregnancy data obtained in these subjects were compared with those in the ten subjects with normal pregnancies. On the basis of the difference between the two groups the hypothesis was tested that early-pregnancy adaptation is already abnormal in subjects that develop impaired fetal growth in the second half of pregnancy.

This thesis starts with a review of the current literature on the adaptations in hemodynamics and volume homeostasis in normal pregnancy and in complicated pregnancy. 


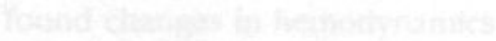

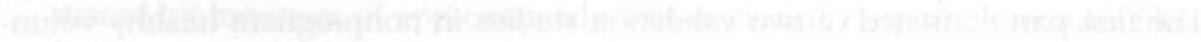

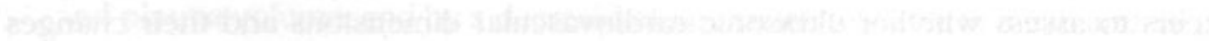

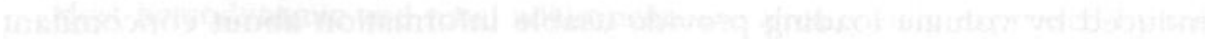

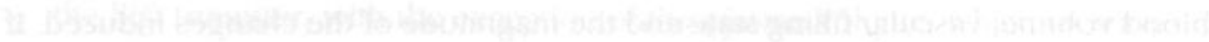

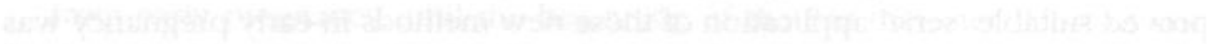

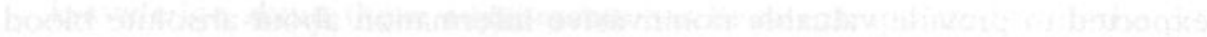

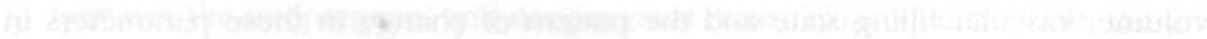

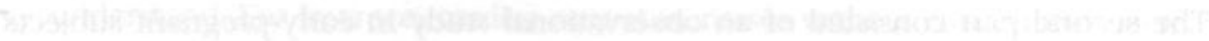

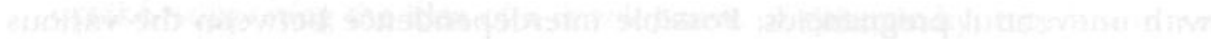

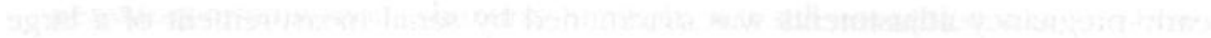


Chapter 2

\section{MATERNAL CARDIOVASCULAR HEMODYNAMIC ADAPTATION TO PREGNANCY}

Johannes J Duvekot $\mathrm{MD}^{1}$, and Louis LH Peeters MD, $\mathrm{PhD}^{2}$.

From the Departments of Obstetrics and Gynecology, St. Clara Hospital, Rotterdam' and University Hospital Maastricht, Maastricht ${ }^{2}$, The Netherlands.

Obstetrical \& Gynecological Survey 1994; in press. 
The maternal cardiovascular system undergoes profound changes during pregnancy. It is useful to study this hemodynamic adaptation thoroughly, not only to assess maternal health during pregnancy in women with cardiovascular disease, but also to predict and possibly treat women with complicated pregnancies. With the development of new and non-invasive techniques it is now possible to determine the patterns in maternal cardiovascular changes from early pregnancy onwards.

\subsection{History}

Our understanding of the circulatory changes in pregnancy has improved with the development of newer and more accurate techniques. The first report on maternal cardiovascular function in pregnancy was published by Lindhard in 1915, and was based on the dye-dilution technique (Lindhard 1915). In this study Lindhard described his experiments with respect to cardiac output during physical exercise. One of his volunteers conceived in the course of his experiments, and cardiac output was determined before, during and after pregnancy. Lindhard observed an increase in cardiac output during pregnancy by as much as $50 \%$. In the first decades of this century foreign gases, usually nitrous oxide or acetylene were used as dilutors. Data obtained in these studies suggested an increase in cardiac output by $45-85 \%$ (Grollman 1932; Stander and Cadden, 1932; Burwell and Strayhorn, 1933). Burwell gave a chronological summary of these early studies in 1938 (Burwell et al., 1938). The limitations and relative inaccuracy of the gas methods led to the development of more invasive techniques. In 1941 the Cournand cardiac catheterisation technique was introduced, which allowed blood sampling from the right side of the heart (Cournand and Ranges, 1941). Cardiac output was calculated on the basis of the wellknown Fick principle. The first studies employing this technique during pregnancy were published in the late fourties (Hamilton 1949; Palmer and Walker, 1949). Most of the errors in the earliest studies were related to incorrect positioning of the catheter to sample mixed venous blood in the pulmonary artery. In addition, the arterial oxygen content was calculated from hemoglobin concentration assuming an oxygen saturation of $95 \%$. After technical revision of this method, Bader published a crosssectional study in 1955 (Bader et al., 1955). In that study, cardiac output reached its peak at about 25 to 27 weeks of gestation, followed by a decline toward term.

In the next decade, improvement of the indicator dilution techniques led to the replacement of the invasive cardiac output determination by means of the Cournand catheter. After the development of continuous photometric dye analysis of arterial blood and capillary blood, assessed by transillumination of the ear, made this method easy to perform (Lees et al., 1967). Above all, it offered the possibility to perform longitudinal studies. In the first longitudinal study during pregnancy, cardiac output was determined monthly in 30 subjects, using the photo-electric ear piece dye-dilution technique. Cardiac output reached its maximum at 24-30 weeks. Afterwards, it decreased and approached non-pregnant levels at term (Walters et al.,1966). 
The first non-invasive technique to measure stroke volume was based on pulse pressure analysis. It was introduced in the $1950 \mathrm{~s}$ in studies performed during pregnancy and during labor (Brehm and Kindling, 1955; Hendricks and Quilligan, 1956; Quilligan and Tyler, 1959). In this technique, stroke volume is assumed to vary as a function of pulse pressure. The application of this method in longitudinal studies in pregnancy required many assumptions to be made, which reduced the accuracy markedly. As a result, this method never gained much popularity for application in pregnancy.

The recognition of the importance of the subject's position for the measurement results was another important development in the elucidation of maternal hemodynamics during pregnancy (Quilligan and Tyler, 1959; Vorys et al., 1961). The first studies, with measurements performed in lateral position, indicated a continuous elevation of cardiac output in the third trimester, rather than a decrease (Lees et al., 1967). The positional effect was assumed to be due to the pooling of blood in the lower extremities, a phenomenon triggered by compression of the inferior vena cava by the "flaccid" pregnant uterus (Vorys et al., 1961).

With the introduction of M-mode echocardiography in the 1960 s and 1970 s, an accurate non-invasive technique became available. However, this method was never properly validated against the generally accepted invasive "golden standard" methods. Validation of this method is particularly important, since realtime ultrasound may overestimate stroke volume because of the dilatation of the left ventricle during pregnancy (Robson et al., 1987d). Stroke volume is calculated from the difference in left ventricular volume between the diastolic and systolic phases of the cardiac cycle. The method assumes the left ventricle to be ellipsoid, with the long axis having twice the length of the short axis. This normal shape of the ventricle is disturbed in pregnancy by changes in the left ventricular dimensions, leading to inaccurate measurements especially in late pregnancy (Fortuin et al., 1972). In 1966 Kubicek introduced a technique based on the measurement of changes in electrical impedance during the cardiac cycle; impedance cardiography (Kubicek et al., 1966). While impedance cardiography is still being discussed as a potentially useful tool during pregnancy (De Swiet and Talbert, 1986; Easterling and Benedetti, 1990a), the refinement of the Doppler technique, supplemented by continuous and pulsed wave Doppler, improved the accuracy of echocardiographic measurements. The latter techniques were validated, both in non-pregnant and pregnant subjects, against the direct and "golden standard" techniques of central hemodynamic measurements, obtained with flow-directed, thermistor-tipped pulmonary artery catheters (Huntsman et al., 1983; Robson et al., 1987d; Easterling et al., 1987). With the development of these new, non-invasive, repeatable, accurate and safe techniques it became possible to study the cardiac output serially in advanced normal as well as in complicated pregnancies. It also offered the possibility to explore the cardiac output changes in early pregnancy (Robson et al., 1989a). Refinement of the invasive techniques employing pulmonary artery catheters renewed interest in its application during pregnancy. Since the first report on this application (Benedetti et al., 1980), central hemodynamic measurements using Swan-Ganz pulmonary artery catheters have been avocated in some studies for the clinical treatment 
of hypertensive disorders in pregnancy (Visser and Wallenburg, 1991). Since as yet no clear-cut central hemodynamic profile for pregnancy induced hypertension is available and hence, no study so far has proved a risk-benefit advantage for such monitoring, this invasive technique is as yet only of limited value in complicated cases of preeclampsia (Cunningham and Lindheimer, 1992; Clark et al., 1986; Clark and Cotton, 1988).

\subsection{Measurement methods}

At present the methods most frequently used to study maternal hemodynamics in pregnancy are the non-invasive techniques of combined cross-sectional and Doppler echocardiography or impedance cardiography. Less frequently used is the invasive technique by Swan-Ganz pulmonary artery thermodilution catheter monitoring.

Combined cross-sectional and Doppler echocardiography: The validity of non-invasive cardiac output measurement by echocardiography, combined with continuous or pulsed wave Doppler, has been demonstrated for the nonpregnant state in various studies by different investigators (Huntsman et al., 1983; Loeppky et al., 1984; Schuster and Nanda, 1984). In this method, stroke volume is calculated as the product of Doppler flow velocity integral, represented by the area under the velocity curve, and the cross-sectional area of the vessel at the site of the flow velocity measurement. In pregnancy, this non-invasive technique has also been validated against central hemodynamic measurements (Lee et al., 1988; Easterling et al., 1987; Easterling et al., 1990b). Although a good correlation (range 0.93-0.98) was found with thermodilution methods, irrespective of whether continuous wave or pulsed Doppler was used, only critically-ill obstetrical patients suffering from severe preeclampsia have been included in the validation studies. The major problems of stroke volume calculation by this method are the choice of most appropriate echographic method and the most suitable site of measurement of the cross-sectional area (CSA) through which the blood flow is measured. Pulsed-wave and continuous-wave Doppler echocardiography are, both in the non-pregnant and pregnant state, considered equally accurate for velocity measurements in the ascending aorta (Ihlen et al., 1984; Mabie et al., 1994). Flows measured over the aortic, pulmonary and mitral valves were found to correlate well, both in the pregnant and the non-pregnant situation. Since measurement at the level of the aortic valve is technically easier (Stewart et al., 1985; Robson et al., 1987d), permitting adequate and reproducible measurement of the CSA, this site is considered the most appropriate for the measurement of cardiac output (Ihlen et al., 1984; Bouchard et al., 1987). In addition, the shape of the aortic valve orifice was found not to differ between diastole and peak-systole (Ihlen et al., 1984). The use of A-mode, M-mode and 2-D imaging for measuring CSA of the aorta have shown adequate correlations with the invasive methods in several validation studies (Ihlen et al., 1984; Gardin et al., 1985; Bouchard et al., 1987).

Impedance cardiography: In this technique the changes in electrical impedance that 
occur in the thoracic cavity during the cardiac cycle are used to calculate stroke volume. Application of a tetrapolar circumferential band-electrode to the head and neck allows continuous measurement of stroke volume. In the non-pregnant state there is some ambiguity concerning the accuracy of this technique. Recently, a modified technique of impedance cardiography has been validated against invasive thermodilution techniques in late pregnancy (Masaki et al., 1989). This study revealed a large underestimation of cardiac output, which had already been described for this technique before (Milsom et al., 1983), and was based on statistical errors (Easterling and Benedetti, 1990a). In contrast with this finding, validation studies in the nonpregnant situation have reported a consistently larger stroke volume when using impedance cardiography (Milsom et al., 1983). The underestimation of stroke volume during pregnancy was demonstrated in three longitudinal studies employing this technique (Atkins et al., 1981; Davies et al., 1986; Heilmann et al., 1993). Cardiac output did not increase significantly throughout most of pregnancy and even decreased near term, to fall below non-pregnant levels. One of the major assumptions of the method is a constant ejection flow rate equal to the initial flow. It is most likely that the flow/time profile changes in the course of pregnancy, since ejection time is substantially reduced in the course of gestation (De Swiet and Talbert, 1986). Determination of stroke volume by the impedance technique is also influenced by intrathoracic fluid volume, thorax configuration and hematological changes, all of which that show physiological changes characteristic of pregnancy (Milsom et al., 1983). This was particularly demonstrated in a study comparing impedance and thermodilution technique in preeclamptic pregnant subjects (Easterling et al., 1989a). It must be concluded from these theoretical and practical considerations that impedance cardiography is at least not suitable for following cardiovascular changes longitudinally during pregnancy. Swan-Ganz pulmonary artery thermodilution catbeter monitoring: Since its introduction into clinical medicine in the 1970 s, the pulmonary artery catheter has become an important instrument in the treatment of critically ill patients. Indications for invasive hemodynamic monitoring in obstetric patients include severe hypertension, unresponsive to conventional antihypertensive therapy, pulmonary edema and oliguria (Clark and Cotton, 1988). The invasive character of this method limits its use in the study of maternal hemodynamics during normal pregnancy. Data on central hemodynamic changes determined with this technique during normal late pregnancy are scarce (Grollman 1932; Clark et al., 1988; Visser and Wallenburg, 1991).

\subsection{Cardiac output, stroke volume and heart rate}

Although it has already been known for more than 70 years that cardiac output rises in pregnancy (Lindhard 1915), it is only in the last decade that it has become feasible to determine the timing and magnitude of this increase. Most of this increase is generally agreed to occur in the first trimester of pregnancy (Walters et al., 1966; Atkins et al., 1981). However, it has become clear only recently that a distinct rise is noticeable even in the first weeks of pregnancy (La Sala et al., 1989; Capeless and Clapp, 1989; Robson et al., 1989a). It has been observed that an initial rise in heart 
rate is followed by an increase in stroke volume during the subsequent weeks (Clapp et al., 1988; Robson et al., 1989a). The physiologic meaning of the sequential rise in heart rate and stroke volume in early pregnancy is still unclear. In mid- and late pregnancy, increases in both stroke volume and heart rate lead to a further rise in cardiac output. A plateau in cardiac output is reached by the end of the second trimester (Bader et al., 1955; Lees et al., 1967; Caton and Banner, 1987; Robson et al., 1989a; Thomson et al., 1993). Afterwards, cardiac output remains at this level until term (Barth 1990; Vered et al., 1991; Thomson et al., 1993). Some studies have been reported a continued rise in heart rate, accompanied by a fall in stroke volume in late pregnancy, without a consistent effect on cardiac output (Mashini et al., 1987; Robson et al., 1989a; Easterling et al., 1990c). Most of the earlier studies reported a decline in cardiac output to non-pregnant levels in the third trimester (Palmer and Walker, 1949; Hamilton 1949; Bader et al., 1955; Walters et al., 1966; Ueland et al., 1969c). Although the absolute values of cardiac output obtained by these authors are largely unreliable, because the methods they used are now obsolete, more recent studies, applying modern techniques, also showed a decrease in cardiac output toward term, due to a fall in stroke volume (Rubler et al., 1977; James et al., 1985; McLennan et al., 1987; Easterling et al., 1990c). All of these studies were performed with the subjects in lateral position during the measurements. Doppler studies in the third trimester in normal pregnant subjects, comparing measurements in lateral and supine positions, have shown no differences in cardiac output near term (James et al., 1985; McLennan et al., 1987; Vered et al., 1991). Heart rate was significantly increased by turning the subjects from lateral to supine (Kinsella et al., 1990) or standing position (Clark et al., 1991) but this was compensated for by a decrease in stroke volume (McLennan et al., 1987; Rawles et al., 1987; Vered et al., 1991). In conclusion, cardiac output decreases due to a fall in stroke volume after the 35 th week, with heart rate remaining constant. The decrease to non-pregnant values found in many studies seems to be due to inaccurate measurement methods.

The magnitude of changes in cardiac output varies enormously in the different studies. Proposed explanations for these differences are: different measurement methods, transversal or longitudinal data, small number of subjects in some studies, differences in reference point for baseline data and measurement points during pregnancy. Because of the large inter-individual differences in cardiac output, only longitudinal studies seem suitable for drawing conclusions on this subject. In all but two studies (Robson et al., 1989a; Capeless and Clapp, 1989) cardiac output measurements in pregnancy were compared with those obtained postpartum. Postpartum and pre-pregnancy values within the same subjects do not differ appreciably (Capeless and Clapp, 1991; Hunter and Robson, 1992). Modern echocardiographic methods, combined with Doppler, have revealed an increase in cardiac output by 35 to $50 \%$ (Robson et al., 1989a; Easterling et al., 1990c; Vered et al., 1991; Thomson et al., 1993), which is in agreement with the earliest reported data (Katz et al., 1978). The large range observed with the same technique may be related to differences in the measurement of the CSA of the aortic outflow tract. Technically and methodologically, an accurate CSA measurement is more difficult to obtain than the Doppler velocity measurement (Ihlen et al., 1984; Bouchard et al., 1987). Minor differences in dia- 
meter have a large impact on CSA, giving rise to measurement errors of as much as $15 \%$ (Ihlen et al., 1984). Although in the non-pregnant state the aortic CSA increases only slightly and not significantly with increasing flow rates (Stewart et al., 1985), the altered structure of connective tissue associated with the increased estrogen levels in pregnancy may lead to increased distensibility of the aortic valve annulus (Beldekas et al., 1981). A proposed concept of a decrease in aortic diameter due to the lower blood pressures during pregnancy has never been substantiated (Hart et al., 1986). Several studies have reported an increase in aortic valve orifice area by 10 to $30 \%$ (Katz et al., 1978; Hart et al., 1986; Robson et al., 1989a; Vered et al., 1991), whereas others have reported no appreciable change (Caton and Banner, 1987; Sadaniantz et al., 1992; Campos et al., 1993; Mabie et al., 1994). Differences in the techniques employed in these studies may have led to this great discrepancy and constitute the major disadvantage of the Doppler flow derived cardiac output measurements. To bypass this possible error, two longitudinal studies measured stroke and minute distance, linear analogues of stroke volume and cardiac output, respectively (Campbell et al., 1985a; McLennan et al., 1987). Maximum increases in stroke and minute distance were $20-34 \%$ and $30-71 \%$, respectively.

Studies performed in the 1940 s, using the Cournand catheterization technique, already established that the arterial-venous oxygen difference decreases during pregnancy, reaching a minimum by the end of the first trimester (Palmer and Walker, 1949; Hamilton 1949; Bader et al., 1955). Recently this phenomenon was also demonstrated in pregnant rats (Gibson et al., 1992). This paradoxical finding of reduced oxygen consumption in a situation of increased flow demands has been attributed to the uterine circulation acting as an arteriovenous shunt (Longo 1983; Sady et al., 1990). Acute closure of the uterine circulation in pregnant rabbits led to hemodynamic changes comparable with closure of an arteriovenous shunt (Valenzuela et al., 1990). In early human pregnancy, however, the development of the uterine circulation as a fraction of total cardiac output is still modest, since uterine blood flow does not begin to increase rapidly until the second half of pregnancy (Assali et al., 1960). Therefore, at least one other factor must be responsible for the early increase in cardiac output.

\subsection{Functional and structural changes in the heart}

As was described above, the data on the gestational changes in the aortic outflow tract orifice are conflicting. There is less disagreement about the changes in the ventricular dimensions with advancing pregnancy. Left ventricular end-diastolic dimension (LVEDD), as measured by M-mode echocardiography, increases in the first trimester (Robson et al., 1989a; Capeless and Clapp, 1989) or during the second trimester (Rubler et al., 1977; Katz et al., 1978; Laird-Meeter et al., 1979), and remains elevated until term. Left ventricular end-diastolic dimension is known to increase with a rise in preload (Nixon et al., 1982; Flessas and Ryan, 1982; Campos et al., 1993). Left ventricular end-systolic dimension (LVESD) may be slightly decreased to below the non-pregnant value in the first trimester (Rubler et al., 1977; Katz et al., 
1978; Robson et al., 1989a; Barth 1990). Afterwards, LVESD either remains unchanged (Rubler et al., 1977; Laird-Meeter et al., 1979) or increases only in the last weeks of pregnancy (Katz et al., 1978; Kuzniar et al., 1983; Robson et al., 1989a; Barth 1990). The observed fall in LVESD in early pregnancy may be due to the increased heart rate, in combination with the increased myocardial contractility which occurs during this period of pregnancy. As a consequence of these changes, left ventricular mass, which can be derived using the formula provided by Devereux and Reichek (Devereux and Reichek, 1977), increases during pregnancy (Katz et al., 1978; LairdMeeter et al., 1979; Robson et al., 1989a; Barth 1990), which suggests myocardial hypertrophy. The cardiovascular status in pregnancy resembles that seen in response to physical training (Robson et al., 1989a). After some months of isotonic exercise, previously untrained athletes develop larger cardiac dimensions, accompanied by a higher cardiac contractility and stroke volume. Cardiac output remains unaltered, however, because of a concomitant proportional fall in heart rate (Lusiani et al., 1986). Although the structural cardiac changes are comparable in pregnancy and after training, these changes are effectuated by entirely different mechanisms. Nevertheless, a comparison between the structural changes in the heart during pregnancy and those found in the above study shows that the hemodynamic demands in pregnancy are consistent with the effects of chronic strain put upon the heart.

Nearly all data on changes in the atrial dimensions during pregnancy have been based on measurements of the left atrium. The validity of echocardiographic measurements of left atrial dimensions has been demonstrated by the good correlation with cineangiocardiograms, used as golden standard (Hirata et al., 1969). The size of the left atrium has been demonstrated to correlate well as an indirect estimate of vascular filling state (Di Donato et al., 1982; Cannella et al., 1988; Rector et al., 1990). During normal pregnancy left atrial diameter begins to increase gradually from early pregnancy on (Robson et al., 1989a), and reaches a plateau by the 30th week (Katz et al., 1978; Vered et al., 1991; Campos et al., 1993). This pattern follows closely the rise in blood volume during pregnancy (Hytten and Paintin, 1963; Pirani et al., 1973). Interestingly, the increase in left atrial diameter starts late in early pregnancy (Robson et al., 1989a), while some studies showed it to be possibly preceded by a decrease with respect to the nonpregnant state (Katz et al., 1978). The further increase in left atrial diameter during pregnancy suggests a rise in both preload and circulating blood volume. Mean circulatory filling pressure is defined as the pressure measured in the circulatory system when the heart is suddenly stopped and the blood rapidly distributed between the arterial and venous beds so that the pressure equalizes throughout the cardiovascular system (Guyton et al., 1973b). The blood volume causing this expanding pressure is called stressed volume. If this stressed volume falls to zero, for example as a result of bleeding, mean circulatory filling pressure also falls to zero. The residual blood volume in the vasculature is then called the unstressed volume. The vascular capacity of the body at a given moment is the sum of the unstressed volume plus the product of the mean circulatory filling pressure and overall vascular compliance (Rothe 1986). Mean circulatory filling pressure minus right atrial pressure represents the pressure gradient for venous return (Prather et al., 1969). Since right atrial pressure and central venous pressure are unchanged during pregnancy (Bader 
et al., 1955; Visser and Wallenburg, 1991) mean circulatory filling pressure can be expected to increase during human pregnancy. During the pregnancies of dogs, guinea pigs and rabbits mean circulatory filling pressure was found to increase (Douglas et al., 1967; Goodlin et al., 1984; Cha et al., 1991). All of these studies were performed in mid- or late pregnancy and data for early pregnancy are unfortunately still lacking. Whether vascular compliance shows any changes during pregnancy in humans is as yet unknown. In guinea pig pregnancy, total vascular compliance was found to be unchanged or increased (Davis et al., 1989; Cha et al., 1991). It is interesting to speculate on the advantages of an increased compliance and capacitance in pregnancy. An increase in unstressed volume would permit a reserve in blood volume that is not hemodynamically active, while increased compliance preserves blood pressure during volume changes (Davis et al., 1989). The ultrasonic dimension of the inferior vena cava has been proposed as another possible indirect estimate of vascular filling state (Cheriex et al., 1989). Although the measurement of the inferior vena cava depends on a healthy right-sided heart function (Mintz et al., 1984; Kirchner et al., 1990), its use in early pregnancy seems promising, especially if one wishes to explore the early changes in maternal vascular filling state. In late pregnancy, the aortocaval compression by the pregnant uterus is likely to affect the inferior vena cava dimensions too much to allow accurate data to be obtained (Kerr et al., 1964; Milsom and Forssman, 1984).It is difficult to determine whether ventricular performance increases during pregnancy, as the latter is strongly influenced by changes in heart rate, preload and afterload (Laird-Meeter et al., 1979). As a parameter for myometrial contractility, the mean velocity of circumferential fibre shortening (mean Vcf) has been introduced. Mean Vcf increases even in early pregnancy, together with the rise in heart rate (Rubler et al., 1977; Robson et al., 1989a). This increase is more pronounced than might be expected from the concomitant rise in heart rate alone (Rubler et al., 1977; Robson et al., 1989a). Mean Vcf is strongly influenced by changes in afterload. Therefore, mean Vcf changes in mid- and late pregnancy are probably unsuitable for providing information on myometrial contractility in advanced pregnancy. Studying systolic time intervals, the estimated time during which the ventricle contracts before the aortic valve opens, may also be used to assess contractility during pregnancy. Reduced systolic time intervals have been reported in early, mid- or late pregnancy (Rubler et al., 1973; Burg et al., 1974; Lim and Walter, 1976). Discrepancies in the results of these studies can be explained from the rather wide time-interval (several weeks) during which the measurements were obtained (Lim and Walter, 1976). Raised myometrial contractility in pregnancy may reflect the inotropic effects of estrogen, as demonstrated in male transsexuals during high-dose estrogen administration (Slater et al., 1986). The lack of increase in cardiac contractility in women receiving exogenous estrogen (ovulation-inducing agents or oral contraceptives) is probably dose-related (Kessler et al., 1980; Veille et al., 1986; La Sala et al., 1989). This was supported by studies in patients subjected to multiple follicular stimulation, which only showed an increase in cardiac contractility in the luteal phase if pregnancy was achieved (La Sala et al., 1989). In addition to a dose-effect, the latter could indicate that estrogen influences ventricular performance only in concert with other pregnancyrelated hormones. 


\subsection{Blood pressure}

There is general agreement about the occurrence of only minor changes in systolic blood pressure in uncomplicated pregnancy. Diastolic blood pressure reaches a minimum in mid-pregnancy, to return to non-pregnant levels near term (Andros and Arbor, 1945; Moutquin et al., 1985; Robson et al., 1989a). It follows that mean arterial pressure, defined as (systolic blood pressure + diastolic blood pressure x 2)/3, is lowest in second trimester (Page and Christianson, 1976). Even by the 8th week of gestation, and possibly even during the late luteal phase (La Sala et al., 1989), mean arterial pressure is decreased relative to the pre-conceptional reference (Clapp et al., 1988). In the normal menstrual cycle, both diastolic and systolic blood pressures are decreased in the luteal phase and rise sharply at the onset of menstruation (Dunne et al., 1991). Blood pressure measurements may be influenced by many external factors (Villar et al., 1989). For this reason, recommendations have been made by the American Heart Association to obtain reliable and reproducible blood pressure measurements (Frohlich et al., 1989). In pregnancy, special precautions have to be taken with respect to the posture of the subject and the choice of the Korotkoff tone to measure diastolic blood pressure (Villar et al., 1989). Blood pressures measured from the brachial artery are highest in the upright and sitting positions, intermediate with the subject in the supine position and lowest when she is lying on one side (MacGillivray et al., 1969; Eskes et al., 1974; Wichman et al., 1984). For practical reasons, most studies measure blood pressure in the sitting position, with the cuff at the level of the heart. It is still debatable whether the diastolic blood pressure is best represented by the value obtained at the phase of muffling of Korotkoff sounds (phase IV) or at their final disappearance (phase V) (Johenning and Barron, 1992). The problem arises here since the difference between phases IV and V is increased during pregnancy. This is probably associated with the hyperkinetic state of the circulation (Villar et al., 1989). Studies in which "golden-standard" intra-arterial measurements are compared with those obtained by a sphygmomanometer show conflicting results in pregnancy (Wichman et al., 1984; Johenning and Barron, 1992). The large measurement error associated with the use of phase IV is undoubtly an important disadvantage of the latter approach (Johenning and Barron, 1992). To overcome this problem, the recent consensus report by the "Working Group on High Blood Pressure in Pregnancy" advocated the recording of both Korotkoff phases 4 and 5, to help ensure that no information is lost and that the development of a rise in blood pressure during pregnancy will be noticed. In recent years, automated blood pressure measurements have been incorporated in clinical medicine. Whether these devices can be used in pregnancy is still controversial (Greer 1993). The first validation studies of these blood pressure measurement machines in pregnancy are promising and show good correlation with readings obtained with a mercury sphygmomanometer (Brown and Adsett, 1991; Shennan et al., 1993; Marx et al., 1993). Adequate correspondance was obtained between the auscultatory and oscillatory methods of measurement for both systolic and diastolic Korotkoff $\mathrm{V}$ values, but the latter only when measured in left lateral tilt position (Franx et al., 1994). The use of Korotkoff IV as diastolic blood pressure is not within acceptable limits for all devices (Greer 1993; 
Franx et al., 1994). However, values of systolic and diastolic blood pressures obtained by the oscillatory method during labor were found to be consistently higher and lower, respectively, than those obtained with an automated device (Marx et al., 1993). Consequently, mean arterial blood pressure remained unchanged. Because of the higher values for diastolic blood pressure, and also for practical reasons, auscultatory measurement still prevails during labor.

\subsection{Systemic vascular resistance}

Systemic vascular resistance, represented by the ratio of cardiac output and mean arterial pressure, decreases to a minimum in mid-pregnancy, followed by a gradual rise until term, similar to the pattern of mean arterial pressure. Since cardiac output remains elevated until term, systemic vascular resistance is also decreased until term (Bader et al., 1955; Walters et al., 1966; Mashini et al., 1987; Robson et al., 1989a; Easterling et al., 1990c). Even by the fifth week of pregnancy, systemic vascular resistance has been found to decreased significantly below the nonpregnant reference (Capeless and Clapp, 1989; Robson et al., 1989a). A significant decrease in systemic vascular resistance was already observed during the late luteal phase in women that subsequently became pregnant (La Sala et al., 1989). This suggests that a lower systemic vascular resistance represents one of the earliest maternal adjustments to pregnancy. The mechanism for this fall in systemic vascular resistance is poorly understood. The earlier suggestion of the uteroplacental circulation acting as an arteriovenous shunt, causing a fall in systemic vascular resistance, seems unlikely. The systemic vascular resistance decreases very early in pregnancy, and data from animal experiments have shown that this shunt is only of moderate size (Longo 1983). A more promising theory involves the presence of one or more circulating substances exerting a vasodilatory effect on arterial and venous vasculature (Schrier and Biner, 1991).

\subsection{Pulmonary circulation and pulmonary arterial pressure}

Data on the adaptation of the pulmonary circulation are scarse. Using a Cournand right heart catheter, Bader measured central hemodynamics longitudinally in all trimesters and observed little change in mean pulmonary arterial pressure during pregnancy (Bader et al., 1955). Because of the higher cardiac output, this would imply a reduced pulmonary vascular resistance during the greater part of pregnancy. These findings have recently been confirmed using Swan-Ganz pulmonary artery catheter monitoring, but these measurements were all confined to the third trimester (Wallenburg 1988; Clark et al., 1989). A longitudinal study of pulmonary circulation during pregnancy was reported by Robson (Robson et al., 1990). In this study the non-invasive method of Doppler echocardiography was used to assess pulmonary pressure and flow. Mean pulmonary artery pressure was calculated from pulsed 
Doppler velocities. No significant change in mean pulmonary artery pressure was demonstrated. This finding implies a large decrease in pulmonary vascular resistance to facilitate the increased flow. Cardiac output may increase by four to six times before pulmonary arterial pressure becomes elevated. The necessary reduction in pulmonary resistance is achieved by the opening of more capillaries and expansion of pulmonary arterioles and capillaries (Guyton 1986b). Despite the increased pulmonary flow, the appearance of chest radiographs remains unchanged in pregnancy. Pulmonary blood flow would have to be at least doubled before one could expect to see changes in vascularity and enlarged pulmonary vessels on chest radiographs (Turner 1975).

\subsection{Multiplet pregnancies}

Three cross-sectional and one longitudinal study have compared cardiovascular hemodynamics in singleton and twin pregnancies (Grollman 1932; Rovinsky and Jaffin, 1966; Veille et al., 1985; Robson et al., 1989b). Three of these studies reported a $15 \%$ larger increase in cardiac output in twin pregnancy. The pattern of change in cardiac output was similar to that in singleton pregnancy. After an increase upto week 20, cardiac output remained constant for the remainder of pregnancy (Robson et al., 1989b). The increased cardiac output was caused primarily by a higher heart rate relative to singleton pregnancy. In one study stroke volume was only increased in the third trimester (Veille et al., 1985). Although a higher mean velocity of circumferential fibre shortening in twin pregnancy suggested improved cardiac contractility, this increase was probably induced by the increase in heart rate (Kass et al., 1987). The increase in left atrial diameter was larger in twin pregnancy than in singleton pregnancy (Robson et al., 1989b), which is in agreement with the larger volume increase in multiplet pregnancies (Rovinsky and Jaffin, 1965). Mean arterial pressure was also shown to have decreased more in mid-pregnancy, as had systemic vascular resistance (Campbell and Campbell, 1985b; Robson et al., 1989b). In pregnant women with a healthy cardiovascular state this chronic extra rise in circulatory demands in multiplet pregnancies does not increase the risk of hemodynamic derailment. However, the greater strain put upon the reserve capacity of the circulation and the consequent larger rise in adrenergic hormones are probably responsible for a higher incidence of pregnancy complications in multiplet pregnancies (Veille et al., 1985; Easterling and Benedetti, 1989b).

\subsection{Hemodynamic changes during labor}

During labor cardiac output has generally been found to increase gradually (Hendricks and Quilligan, 1956; Ueland and Hansen, 1969b; Robson et al., 1987a). The magnitude of the rise in cardiac output between contractions varies from $12 \%$ to $31 \%$. This increase is mainly due to a rise in stroke volume. Reports on changes in heart rate have been inconsistent. Most studies have only observed an increase in 
heart rate following contraction (Adams and Alexander, 1958; Robson et al., 1987a). The increase in cardiac output during labor is generally thought to result from anxiety and pain (Hendricks and Quilligan, 1956), which is supported by the observation that cardiac output increases less in the presence of any form of analgesia (Ueland and Hansen, 1969b). Maternal posture also influences hemodynamics during labor (Winner and Romney, 1966; Ueland and Hansen, 1969b). Between contractions, cardiac output is higher in lateral than in supine position, because of an increased stroke volume (Ueland and Hansen, 1969a). During contractions, cardiac output is additionally increased (Ueland and Hansen, 1969a; Robson et al., 1987a), possibly as a result of enhanced uterine venous drainage during contractions, which raises venous return. Systolic blood pressure increases during labor by a maximum of $35 \mathrm{mmHg}$ and diastolic blood pressure by a maximum of $25 \mathrm{mmHg}$ (Winner and Romney, 1966; Robson et al., 1987a). It is obvious that labor and delivery represent a period of considerable additional hemodynamic demands. This poses special problems in patients with an impaired cardiovascular function. Two thirds of maternal deaths from heart disease occur during or shortly after labor (Conradsson and Werko, 1974).

\subsection{Hemodynamic changes during the puerperium}

Immediately after delivery, cardiac output and stroke volume increase sharply (Hendricks and Quilligan, 1956; Adams and Alexander, 1958; Ueland and Hansen, 1969b; Newman 1982; James et al., 1989). After reaching a maximum value at 10-15 minutes postpartum, cardiac output begins to decline, to reach prelabor values one hour after delivery (Robson et al., 1987a; James et al., 1989). In most older studies the postpartum normalisation of cardiac output to return to values prior to the beginning of contractions was found to require about 24 hours (Adams and Alexander, 1958; Kjedsen 1979; Newman 1982). The longer recovery period in the latter studies may be attributable to the older methodology employed to measure cardiac output, and to postural factors (Robson et al., 1987a). Heart rate has been reported either to decrease sharply in the first minutes postpartum (Ueland and Hansen, 1969b) or to remain stable (Robson et al., 1987a; James et al., 1989) depending on the posture of the patient. Compared to non-pregnant values, heart rate remains elevated during the first 24 hours, declining afterwards until the 10th postnatal day (Robson et al., 1989c). Left atrial diameter remains increased for at least 48 hours postpartum, which is consistent with an increase in venous return in early puerperium (Robson et al., 1987). Obviously, the changes in maternal hemodynamics are different during the first 48 hours postpartum if the preceding labor was complicated by excessive hemorrhage (estimated blood loss greater than $500 \mathrm{~mL}$ ) (Robson et al., 1989c). These subjects differ from a control group by a reduced stroke volume and a rise in heart rate, resulting in no net effect on cardiac output. When measured in left lateral position, blood pressure was found to fall in the first two days postpartum, followed by a return to levels prior to delivery (Robson et al., 1987a; Robson et al., 1987b; Robson et al., 1989c; Capeless and Clapp, 1991). In patients who had not been in labor and who were delivered by a primary cesarean section, no postpartum changes in blood pres- 
sure were observed (James et al., 1989). This supports the concept of a transient stress-related rise in blood pressure during labor. The mechanisms of the changes in maternal hemodynamics in early puerperium are still poorly understood. The old concept of a so-called squeezing out of the uterus may account for an increase in blood volume, but blood loss seems to compensate this effect at least partly. Data on blood volume changes immediately postpartum indicate no transient rise in the early puerperium, but even show a decrease in blood volume by as much as 10 percent one hour postpartum (Ueland 1976). In a more modern concept, the abrupt change in endocrine environment may induce changes in both cardiovascular hemodynamics and volume homeostasis. Nevertheless, the exact role of the disappearance of the hormones of pregnancy after delivery has not yet been elucidated.

In the postpartum period the accelerated maternal circulatory function normalizes gradually. These changes have been the subject of several serial studies (Katz et al., 1978; Walters et al., 1966; Atkins et al., 1981; Mashini et al., 1987; Robson et al., 1987b; Sady et al., 1990; Capeless and Clapp, 1991). However, the period needed to return to preconception values varies markedly between these studies. The most reliable data are provided by two longitudinal studies employing more measurement points and using the non-invasive method of echocardiography (Robson et al., 1987b; Capeless and Clapp, 1991). One of these studies compared the postpartum data with preconceptional data for the same individuals (Capeless and Clapp, 1991). Stroke volume and cardiac output were found to decrease rapidly in the first two weeks postpartum, thereafter continuing to decrease until 24 weeks postpartum before stabilizing (Robson et al., 1987b; Capeless and Clapp, 1991). The reduced arterial-venous oxygen difference during pregnancy normalizes in concert with the return of cardiac output to prepregnancy values (Sady et al., 1990). Heart rate returns to pre-conceptional levels within the first 10 postnatal days (Robson et al., 1987b; Robson et al., 1989c; Capeless and Clapp, 1991). The same pattern of recovery has been observed for the left atrial dimension (Robson et al., 1987b). This finding indicates a relatively rapid normalisation of circulating blood volume and preload. Mmode measurements of the left ventricle have shown that a significant reduction in left ventricular end-diastolic dimension two weeks postpartum was not parallelled by any change in left ventricular end-systolic dimension (Robson et al., 1987b). These changes are in agreement with the still elevated stroke volume. In the subsequent 4-6 months both dimensions merged toward the non-pregnant values. Consequently, left ventricular mass decreased until postnatal week 12. Myocardial contractility decreased gradually over the first two weeks postpartum to stabilize afterwards (Robson et al., 1987b). At 12-24 weeks postpartum several hemodynamic variables had not reached a steady statc. This observation suggests that not all cardiovascular parameters returned to their prepregnancy baseline. It is possible that the time elapsed between preconception and postpartum modified a subjects' cardiovascular status. It seems, however, that pregnancy induced structural cardiac and vascular changes. Robson compared cardiovascular data of a control group with data obtained from subjects 24 weeks postpartum. The postpartum group showed a mild residual hypertrophy and lower cardiac contractility (Robson et al., 1987b). Data from animal experiments have also suggested a remodelling of the cardiac ventricle and venous compartment during 
pregnancy (Hart et al., 1985; Davis et al., 1989).

In conclusion, most cardiovascular parameters return to baseline values within 2 weeks postpartum. The stroke volume, cardiac output and left ventricular dimensions require a longer period and probably do not completely recover to pre-conceptional values. This emphasizes the potential error that can be made in the interpretation of postpartum data, it the latter are used as a reference for the baseline state.

\subsection{Hemodynamic changes during pathological pregnancies}

Manifest symptomatology in pregnancies complicated by preeclampsia and/or intra-uterine growth retardation is preceded by a long subclinical period. The onset for these disorders is thought to lie much earlier in pregnancy than its clinical presentation. In the first weeks of pregnancy the spiral arteries of the uterus are converted into utero-placental arteries. This proces is described as the so-called "physiological changes" (Brosens et al., 1967). This vascular phenomenon is thought to evolve in two stages: first the conversion of the decidual segments of the spiral arteries by a wave of endovascular trophoblast migration in the first three weeks after implantation and, after a period of no demonstrable change, a second wave involving the myometrial segments of the spiral arteries between the 14 th and 18th weeks (Khong et al., 1986). In complicated pregnancies the second wave of the endovascular trophoblast invasion shows a defective development (Brosens et al., 1967; Khong et al., 1986). Nevertheless, these changes in the spiral arteries of the placental bed are not pathognomonic for preeclampsia or intra-uterine growth retardation, since they are not present in all cases (Pijnenborg et al., 1991). Since these histopathological abnormalities have been detected in the beginning of the second trimester, it seems worthwhile to investigate whether this disturbance is preceded by abnormal maternal hemodynamic adaptation in the earlier stages of pregnancy. Results from such studies could be used for two purposes: early detection of abnormalities and, if possible, "causal" treatment of pregnancy disorders. Studies of the latent stage of preeclampsia and/or IUGR are scarce, and most of these reports are limited to the description of the pattern of change in blood pressure. A rise in blood pressure or an elevated mean arterial pressure in the second trimester have been identified as early signs of impending preeclampsia (Page and Christianson, 1976; Moutquin et al., 1985). However, the screening value of midtrimester blood pressure measurement is limited by its low predictive value. Recently, this method has been assessed to be a predictor of at most transient hypertension, rather than preeclampsia (Dekker and Sibai, 1991). However, blood pressure shows distinct differences in preeclamptic pregnancies. In a longitudinal study by Easterling a group of initially healthy nulliparous women were followed from week 10 of gestation to six weeks postpartum (Easterling et al., 1990c). Nine women eventually became preeclamptic. These women had a consistently higher mean arterial pressure throughout their entire pregnancy than 89 subjects with uncomplicated pregnancies. This observation was consistent with that in other longitudinal studies on blood pressure in preeclamptic sub- 
jects (Gallery et al., 1977; Moutquin et al., 1985; Reiss et al., 1985). Postpartum, the difference in mean arterial pressure between the two groups disappears rapidly. Unfortunately, in this study the demographic characteristics of the subjects differed between the two groups with respect to weight. The preeclamptic subjects were heavier and therefore had a larger body surface area. Another shortcoming in this study was the large number of missing data, especially in the first and second trimesters.

The other hemodynamic parameters in Easterling's study (Easterling et al., 1990c) show remarkable differences in the subclinical period prior to advanced preeclampsia. Cardiac output and cardiac index, which compensated for the demographic differences between the two groups, were consistently higher in the preeclamptic group, as a result of an elevated heart rate. This finding contrasts with another study, which reported a decreased heart rate in preeclamptic patients during the third trimester (Kuzniar et al., 1983). However, the power of the latter observation was limited, since in the study averaged the heart rate from all the measurements made during the third trimester prior to analysis. The persistent tachycardia in Easterling's preeclamptic patients can be explained as the direct effect of a chronically raised symphathetic tone and the indirect effect of a relative vascular underfill. Neither stroke volume nor total peripheral resistance differed between the two groups, not even during the period of clinical disease. Six weeks postpartum, cardiac output was still higher and total peripheral resistance lower in the preeclamptic group (Easterling et al., 1990c). The findings in Easterling's study are in agreement with the so-called hyperdynamic disease model (Easterling and Benedetti, 1989b). According to this model, endothelial injury, giving rise to the clinical manifestations of preeclampsia, is the result of accelerated flow in the entire microcirculation. No other studies have so far reported maternal hemodynamics in this latent phase of preeclampsia or postpartum. To our knowledge, no studies have been reported that explored the maternal hemodynamics prior to manifest fetal growth retardation or premature labor. The concomitantly disturbed volume homeostasis in these pregnancies (Gibson 1973; Croall et al., 1978; Goodlin et al., 1981; Koller 1982; Huisman and Aarnoudse, 1986) is likely to have an impact on the maternal cardiovascular status. At present it is still obscure whether the physiologic volume increment is already disturbed from the beginning of pregnancy or whether plasma volume is reduced secundarily and preceeds the clinical manifestations. Only in preeclamptic pregnancies is there strong evidence that plasma volume contracts after an initially normal increase during the first and second trimesters (Gallery et al., 1979; Hays et al., 1985a).

Many reports have described central hemodynamics during advanced preeclampsia. Cardiac output, peripheral vascular resistance and left ventricular filling pressures differ markedly between various studies (Benedetti et al., 1980; Strauss et al., 1980; Rafferty and Berkowitz, 1980; Phelan and Yurth, 1982; Groenendijk et al., 1984; Clark et al., 1986; Cotton et al., 1988; Mabie et al., 1989; Hjertberg et al., 1991). The hemodynamic profile of preeclamptic patients ranges from a high-output, low-resistance hemodynamic state to one of a low output and high resistance. Proposed explanations for these differences include: small number of patients in each study, variable severity and duration of preeclampsia, underlying medical problems, therapeutic 
intervention before hemodynamic monitoring and absence of a suitable reference group (Mabie et al., 1989). Only one study included an adequate number of preeclamptic patients enrolled in a standardized manner. Visser described 87 patients who had received no treatment before the start of invasive hemodynamic measurements (Visser and Wallenburg, 1991). She compared the data from this group of patients with those obtained in a group of ten normal pregnant volunteers. The preeclamptic subjects were characterized by a uniform pattern of low cardiac index, high systemic vascular resistance index and normal filling pressures. The hemodynamic profiles at entry into the study varied widely in previously treated patients (Visser and Wallenburg, 1991). If preeclampsia was found to be complicated by pulmonary edema, left ventricular filling pressure, reflected by the pulmonary artery wedge pressure, was as a rule elevated (Strauss et al., 1980; Benedetti et al., 1985; Mabie et al., 1989). Pulmonary edema may develop in response to either of three mechanisms: a decrease in colloid osmotic pressure, cardiac failure (iatrogenic volume overload and/or left ventricular failure) or increased (pulmonary) capillary permeability (Benedetti et al., 1985; Clark and Cotton, 1988). Preeclampsia may involve all three mechanisms. Since no clear-cut risk benefit advantage for invasive hemodynamic monitoring is present, this method should be employed only in a small minority of the patients (Clark and Cotton, 1988; Cunningham and Lindheimer, 1992).

Echocardiographic studies do not lead to stimulation of the sympathetic nervous system as do invasive measurements. Therefore, they are considered to provide a better reflection of the hemodynamic function in basal conditions (Degani et al., 1989). Echocardiographic studies in preeclamptic patients have provided evidence for an elevated total peripheral resistance and a reduced cardiac output (Easterling et al., 1987; Degani et al., 1989; Kuzniar et al., 1992). Similar results have been obtained by impedance echocardiography (Heilmann and Siekmann, 1989). In a recent study cardiac contractility was found to be similar between normotensive and preeclamptic subjects (Lang et al., 1991). This reflects an appropriate response to the increased afterload and rules out an abnormality in ventricular contractile state in these patients. Preeclamptic subjects have also been found to differ from normotensive controls by increases in left ventricular posterior-wall thickness and interventricular septal thickness (Larkin et al., 1980; Kuzniar et al., 1983; Thompson et al., 1986; Degani et al., 1989; Nisell et al., 1992). These changes were more pronounced in pregnant patients with chronic hypertension. In hypertensive subjects mean left ventricular mass was increased throughout pregnancy compared to both normotensive and preeclamptic subjects (Thompson et al., 1986). The cardiac structural changes in hypertensive subjects seem to be influenced by the nature of the hypertension. Women with high-resistance hypertension differed from those with low-resistance hypertension by a difference in aortic diameters. The former group had smaller diameters, while women with high-resistance hypertension showed larger aortic diameters during pregnancy. After the institution of antihypertensive therapy the aortic diameters in both groups returned to values within the normal range (Easterling et al., 1991).

Several investigators have advocated the use of plasma volume expansion as an adjuvans in the management of preeclampsia (Belfort et al., 1989). This therapy is based 
on the fact that hypovolemia seems to precede the rise in blood pressure, which points to the hypothesis that intravascular volume depletion is the primary event. This hypovolemia could induce a secondary compensatory increase in systemic vascular resistance. Not only is plasma volume expansion thought to avoid or reduce the systemic vascular resistance, but also to ameliorate the usually marginal uteroplacental perfusion (Cloeren and Lippert, 1972; Larkin et al., 1980; Sibai et al., 1983). In severe preeclampsia, acute plasma volume expansion may improve the hemodynamic stability (Wasserstrum 1991). Potential hazards of this therapy are serious maternal side effects, such as cerebral edema, cardiac failure and pulmonary edema (Wasserstrum 1991; Nisell et al., 1992). The hemodynamic response to plasma expanders during preeclamptic pregnancy includes an increase in cardiac output in association with a reduction in systemic vascular resistance, while blood pressure does not change appreciably (Groenendijk et al., 1984; Clark et al., 1986; Belfort et al., 1989; Wasserstrum et al., 1989; Stratta et al., 1991; Nisell et al., 1992; Lowe et al., 1993; Grunewald et al., 1994). A similar effect has been seen in healthy normotensive pregnant and nonpregnant women, and animals (Robinson et al., 1966; Boekkooi et al., 1991; Nisell et al., 1992). The physiologically expected decrease in mean arterial pressure after volume expansion was only observed when volume increments were rapid and abundant (Wasserstrum et al., 1989; Boekkooi et al., 1991). In only one study a longlasting beneficial effect was obtained from plasma expansion (Gallery et al., 1981). However, in this uncontrolled study stable plasma protein solution was used which may contain vasodilating substances, accounting for the decrease in blood pressure. The possible benefits of the infused plasma expanders, including an indirect improvement of the uterine blood flow, are transient (Cunningham and Lindheimer, 1992). Repeated albumin transfusions administered to preeclamptic women resulted only in a minority of patients an improvement in uteroplacental and fetoplacental blood flow. This effect lasted for maximal one day (Stratta et al., 1991). It is questionable whether pregnancy can be extended long enough to compensate for the disadvantages of the method, because (intermittent) volume supplementation can only be accomplished under invasive central hemodynamic surveillance. So far, plasma expansion has not been shown to be of benefit for maternal well-being and should therefore not be employed postpartum.

To our knowledge, maternal hemodynamics in pregnancies complicated by fetal growth retardation has only been studied in late pregnancy, when the impaired fetal growth had already become manifest (Nisell et al., 1988; Veille et al., 1991; Rosso et al., 1993). As early as the 1950s, a correlation was presumed to exist between heart volume, assessed by two-dimensional chest radiographs, and fetal growth (Unnerus 1959; Raiha 1964). This finding was later refuted, but heart volume measurements in this study were performed postpartum (Hedberg and Radberg, 1962). Using M-mode echocardiography, no differences in left ventricular size and function were found between 19 women with IUGR pregnancies and 79 women with normal pregnancies near the end of gestation (Veille et al., 1991). In another, smaller study a lower cardiac output was reported in the IUGR group (Rosso et al., 1993). Using invasive hemodynamic measurements in patients with pregnancy-induced hypertension, subjects that delivered from growth retarded babies were found to have a reduced car- 
diac output and stroke volume compared to the women that delivered from appropriate-weight infants (Nisell et al., 1988). It is conceivable that uterine blood flow in IUGR pregnancies is limited near term, which implies decreased flow demands, probably resulting in a smaller cardiac output. In animal studies uterine blood flow correlated well with placental and fetal weight (Owens et al., 1986).

\subsection{The initiation of cardiovascular changes in pregnancy}

The mechanisms inducing the cardiovascular changes in human pregnancy have been debated since the first study on this subject was reported in 1915 (Lindhard 1915). Because of the recent increase in methodological possibilities, it has become feasible to explore noninvasively the hemodynamic adaption in human pregnancy. However, the inability to perform in vivo studies at the trophoblast-decidual interface probably obstructs the identification of the exact initiating mechanisms. Over the years, the following important mechanisms have been proposed: 1 . increased metabolic demands of pregnancy and especially of the uterus and chorioplacental bed; 2 . hemodynamic effects of the hypervolemia in pregnancy; 3 . the uteroplacental circulation acting as an arteriovenous shunt (Bader et al., 1955; Walters and Lim, 1975; Longo 1983). The first mechanism is opposed by the finding that oxygen consumption increases gradually throughout pregnancy until term without a parallel increase in cardiac output (Lees et al., 1967). The observation that the early increase in cardiac output and stroke volume precedes the blood volume expansion is incompatible with the second assumption. The fact that the rise in cardiac output develops in a period when the intervillous circulation has not yet developed, is incompatible with the last assumption. Studies in early human and baboon pregnancy have given rise to a new hypothesis which creates a central role for an initial generalized vasorelaxation, resulting in a state of relative vascular underfill (Phippard et al., 1986; Robson et al., 1989a; Schrier and Biner, 1991). This vasorelaxation is supposed to affect both the arterial and venous vascular compartments. The fall in arterial blood pressure and afterload leads to a rise in cardiac output, primarily through an increase in heart rate (Robson et al., 1989a). Together with the possibly concomitant fall in venous tone, leading to increased venous complicance, this activates volume retaining mechanisms such as the renin-angiotensin axis and the osmoregulatory system. Whether the venous system is actually involved in this early generalized vasodilatation is still obscure, but increased venous compliance has been found at least in the later stages of pregnancy (Goodrich and Wood, 1964; Barwin and Roddie, 1976). The induced volume retention enhances preload and stroke volume. The arterial vasodilatation may also be associated with an increased renal blood flow. After reaching a rebalanced vascular filling, the initial vasodilatation is maintained during pregnancy. This hemodynamic condition is somehow responsible for the resetting of the baroreceptors (Leduc et al., 1991). Several assumptions have been put forward about the nature of this potent, pregnancy-related vasodilator. Prostaglandins have strong vasodilatory effects (Broughton Pipkin et al., 1987), but are unlikely candidates for involvement in 
this early-pregnancy vasodilation, since cyclooxygenase inhibitors have been shown to be unable to neutralize the rise in glomerular filtration rate in midpregnancy in rats (Conrad and Colpoys, 1986). Neither are the pregnancy hormones, such as estrogen, progesterone, prolactin, hCG and hPL, alone or in combination able to mimick the fall the fall in vascular tone observed in early pregnancy as has been shown in animal studies (Barron 1987). A more promising mediator for the vascular effect is the endothelial-derived relaxing factor, of which nitric oxide is one form (Katz 1988; Ramsay et al., 1994). This molecule exerts a strong vasodilatory effect on the resistance vessels. Endothelial-derived relaxing factor acts as an endogenous nitrovasodilatator. Its release is stimulated by neuropeptides and agents released during platelet activation and thrombosis. The release of nitric oxide is stimulated, among other factors, by calcitonin gene-related peptide, which is distributed in the perivascular nerves. Calcitonin gene-related peptide levels in the plasma are increased throughout pregnancy (Stevenson et al., 1986). Inhibition of endothelium-derived relaxing factor by free hemoglobin has been hypothesized to be involved in the pathogenesis of preeclampsia (Sarrel et al., 1990). The study of possible links between the processes evolving at the trophoblast interface and the enhanced release of factors that raise endothelium-derived relaxing factor forms the next step in the unraveling of the presumed generalized vasodilatation in early pregnancy.

\subsection{Conclusions}

Maternal cardiovascular adaptation to pregnancy involves enormous changes. Cardiac output increases in early pregnancy, initially as a result of an increased heart rate, soon followed by an increased stroke volume. Cardiac output continues to increase until midpregnancy, and remains stable afterwards, with a possible small decline in the last weeks of pregnancy. Blood pressure decreases in early pregnancy, reaching a minimum in midpregnancy, then returning to baseline levels at term. Consequently, peripheral vascular resistance is reduced throughout pregnancy. Myocardial contractility appears to be increased during all trimesters of pregnancy, thus gradually provoking the development of a mild ventricular hypertrophy. The increase in preload, which develops in concert with the increment in blood volume, leads to an increase in left atrial diameter, which also begins during early pregnancy. During labor, both cardiac output and blood pressure increase. After delivery, cardiac output initially increases, but begins to decrease within the first hour to reach baseline levels two weeks postpartum. Most cardiovascular parameters show their greatest changes within two weeks postpartum. Five months postnatally, only a mild residual ventricular hypertrophy persists. 


\section{Chapter 3}

\section{RENAL HEMODYNAMICS AND VOLUME HOMEOSTASIS IN PREGNANCY}

Johannes J Duvekot $\mathrm{MD}^{1}$, and Louis LH Peeters MD, $\mathrm{PhD}^{2}$.

From the Departments of Obstetrics and Gynecology, St. Clara Hospital, Rotterdam' and University Hospital Maastricht, Maastricht ${ }^{2}$, The Netherlands.

Obstetrical \& Gynecological Survey 1994; in press. 
In the nonpregnant state blood volume is kept within narrow limits, regardless of daily fluid intake (Guyton 1986c). This characteristic is most likely preserved during pregnancy, when the extracellular compartment accumulates 6 to 8 litres of extra fluid and plasma volume increases by over $50 \%$ (Gallery and Brown, 1987). The associated profound adjustment in the regulation of volume homeostasis has been the subject of extensive clinical and physiological research. Extensive reviews on volume homeostasis in pregnancy have been published in recent years (Walters and Lim, 1975; Davison 1984a; Longo and Hardesty, 1984; Gallery and Brown, 1987; Baylis and Davison, 1990b; Lindheimer et al., 1991a).

\subsection{Blood volume}

Until the third trimester of human pregnancy, plasma volume and red cell mass increase together, but at different rates (Chesley 1972). The first attempt to quantify blood volume in the pregnant state dates back to 1854 (Welcker 1854), when the German scientist Welcker, measured blood volume in pregnant mice. The animals were exsanguinated and their vascular system washed out. Surprisingly, he noted a lower blood volume in pregnant animals, but this was related to the methodology employed. Blood volume was expressed per 100 grams of body weight, but not corrected for the weight of the products of conception. Later studies using indicator dilution techniques unanimously supported a larger blood volume in pregnancy, a phenomenon generally termed as "plethora of pregnancy". The data on the magnitude of the rise in blood and plasma volume during pregnancy reported in the period before 1972 varied widely (Chesley 1972). Careful analysis of these data suggested that a large proportion of this variation was related to the cross-sectional study design, to differences in methodology and to heterogeneity of the study population. By "correcting" as much as possible for these confounding factors, Chesley calculated an average increase in plasma volume of $42 \%$ and an average increase in total red cell mass of $24 \%$ (Chesley 1972). It goes without saying that reliable experimental data regarding the exact rise in plasma and red cell volume can only be obtained in a longitudinal study involving independent measurement of plasma and red blood cell volume, and performed in a study population selected on the basis of the following inclusion criteria: subjects standardized for gestational age, weight, stature, race, salt intake, parity, posture during measurements, and an uneventful pregnancy resulting in the birth of an appropriately grown infant. So far, two studies have measured plasma volume and total red cell volume together in the same subjects (Caton et al., 1951; Verel et al., 1956).

\subsection{Plasma volume}

Plasma volume is usually measured by indicator dilution. Commonly used indicators are albumin, labeled with a dye, T-1824 or Evans' Blue dye, or a radioactive isotope such as ${ }^{131} \mathrm{I}$ or ${ }^{125} \mathrm{I}$. Because of their size and hydrophilic nature, the transplacental transfer of these indicators is probably negligible. A major shortcoming of 
the dilution techniques is their large measurement error, which is probably smallest when a two-wavelength method or polyethyleneglycol method is used (Brown et al., 1992a). The latter techniques have not been used in pregnancy yet.

During the first 30 weeks of pregnancy plasma volume increases gradually by approximatly $1250 \mathrm{ml}$ and is maintained at this level until term (Pirani et al., 1973; Whittaker and Lind, 1993). In earlier studies, plasma volume was found to decline in the final 6-8 weeks of pregnancy (Hytten and Paintin, 1963; Rovinsky and Jaffin, 1965). However, more recent studies found the near-term decline in blood volume to be entirely due to a postural effect. The gestational rise in plasma volume becomes measurable by an amenorrhea of 7-12 weeks (Whittaker and Lind, 1993), an observation already reported twenty-five years ago (Lund and Donovan, 1967).

In multiplet pregnancy plasma volume increases by a larger fraction than in singleton pregnancies (Pritchard 1965; Rovinsky and Jaffin, 1965; MacGillivray et al., 1971; Thomsen et al., 1994), the increase being proportional to the number of fetuses (Fullerton et al., 1965; Rovinsky and Jaffin, 1965). That the presence of a fetus is not essential for blood volume expansion is emphasized by the 3-53\% increase in blood volume found in eight women with a hydatiform mole (Pritchard 1964). Both the maximum increment in plasma volume (Hytten and Paintin, 1963; Gibson 1973; Arias 1975; Ueland 1976) and the maximum plasma volume (Duffus et al., 1971; Pirani et al., 1973; Brown et al., 1992b) correlate closely with newborn weight, even if fetal growth is impaired. This covariation supports the view that optimal maternal adaptation, reflected in an adequate plasma volume expansion, and intrauterine growth have a common denominator. This is suggested by the observation that pregnancies in subjects with a relatively low prepregnant plasma volume are frequently characterized by a combination of subnormal plasma volume expansion and impaired fetal growth (Croall et al., 1978). The seemingly obvious observation of a more pronounced rise in plasma volume in multigravidas is based on the difference in plasma volume between first and subsequent pregnancies in the same subjects, although these were measured in supine and lateral positions, respectively (Campbell and MacGillivray, 1972). However, this study suggested a significant effect on parity only at 38 weeks gestation. Later studies in which the measurement of plasma volume in parous and multiparous women was standardized failed to substantiate this observation (Whittaker and Lind, 1993). It is therefore likely that differences in magnitude of plasma volume expansion between the first and subsequent pregnancies are small.

\subsection{Red cell mass}

Red cell mass can be measured by in-vitro labeling of an aliquot of the subject's own blood cells with ${ }^{51} \mathrm{Cr}$ or with ${ }^{32} \mathrm{P}$. After reinfusion, the red cell mass can be calculated from the degree of dilution. More recently, the old method of labeling hemoglobin with carbon monoxide has been reintroduced (Thomsen et al., 1993).

In spite of concomitant binding of carbon monoxide to myoglobin and other body pigments besides hemoglobin (Letsky 1990), this method is claimed to be as accurate as 
the classical dilution techniques (Fukui and Shigemi, 1989; Thomsen et al., 1991; Thomsen et al., 1993). An important advantage of this method is its suitability for performing serial measurements to monitor rapid changes in red cell volume (Fukui and Shigemi, 1989). In pregnancy, one should keep in mind that transplacental loss of carbon monoxide to the fetal compartment may lead to overestimation of maternal red cell mass (Letsky 1990). The gestational increase in total red cell mass found in various studies ranges from 17 to $40 \%$, with a mean of $24 \%$ (Chesley 1972; Thomsen et al., 1993). Iron supplementation may influence this increase in red cell mass. Crossed-sectional studies in second and third trimester human pregnancy suggest a similar pattern of increase in red cell mass as that reported for plasma volume (Lund and Donovan, 1967). Because of the similar relative increases in maternal weight and red cell mass during pregnancy, the red cell mass per kg maternal weight changes little in the course of pregnancy (Lund and Donovan, 1967). The physiological hemodilution and associated relative anemia in the first half of pregnancy is likely to provide the stimulus for the accelerated red cell production. Elevated erythropoietine levels in pregnancy support this concept (Mansasc and Jepson, 1969). The pattern of change in hematocrit during pregnancy is characterized by a gradual fall until week 30 , followed by a gradual rise afterwards (Koller 1982; Peeters and Buchan, 1989). Obviously, this pattern is the resultant of the concomitant changes in plasma and red cell volume. The physiological hemodilution in pregnancy is associated with a decrease in whole blood viscosity (Peeters and Buchan, 1989) which may be beneficial for the intervillous perfusion (Peeters et al., 1987).

\subsection{Blood volume changes during labor and the puerperium}

Following a normal labor without excessive blood loss (less than $500 \mathrm{ml}$ ), blood volume decreases sharply over the first 24 hours (Ueland 1976). The further decline in the remainder of the puerperium was too slow to be appreciated by standard dilution techniques in cross-sectional studies (Chesley et al., 1959; McLennan and Lowenstein, 1959; Landesman and Miller, 1963; Pritchard 1965; Ueland 1976). The nonpregnant level is reached by 6-9 weeks postpartum (Lund and Donovan, 1967). The initial decline in total blood volume is probably related directly to the smaller size of the vascular bed. The adjustments in volume homeostasis in the first 3 days after labor seem to include a transient rise in plasma volume as well (McLennan and Lowenstein, 1959; Landesman and Miller, 1963). Such a phenomenon is supported by concomitantly elevated $\alpha$-ANP levels (Steegers et al., 1987; Duprez et al., 1989) and may be associated with the accelerated removal of excess extracellular fluid in this period. After intrapartum hemorrhage the interstitial compartment provides a readily available fluid source which can be mobilized to restore the vascular filling state, although of course at the cost of a more pronounced postpartum fall in hematocrit (Ueland 1976,; Taylor and Lind, 1981). Finally, reduced hematocrit levels 5-7 days post delivery are either preceded by excessive intrapartum hemorrhage or poor blood volume expansion in the preceding pregnancy (Chesley 1972; Letsky 1990). 


\subsection{Blood volume changes in pathological pregnancies}

It has been delineated above that neonatal weight varies as a function of the preceding gestational rise and the final peak in plasma volume. It has even been suggested that nonpregnant plasma volume is decreased in women giving birth to small-for-dates newborns (Croall et al., 1978). Subnormal plasma volume expansion is not only observed in pregnancies complicated by fetal growth retardation (Hytten and Paintin, 1963; Gibson 1973; Pirani et al., 1973; Rosso et al., 1993; Salas et al., 1993). The incidence is also increased in pregnancies complicated by hypertensive disorders (Liley 1970; MacGillivray et al., 1971; Duffus and MacGillivray, 1972; Arias 1975; Gallery et al., 1979; Goodlin et al., 1981; Sibai et al., 1983), prematurity (Raiha 1964; Goodlin et al., 1981), oligohydramnios (Goodlin et al., 1981; Goodlin et al., 1983a) and maternal smoking (Pirani and MacGillivray, 1978). In contrast, pregnant diabetics with uneventful pregnancy show a normal rise in plasma volume (Whittaker and Lind, 1993). In pregnant subjects with subnormal plasma volume expansion, the systolic flow murmur is often absent and significant dependent ankle edema is observed more frequently (Goodlin et al., 1983b). Furthermore, their hemoglobin concentration is usually already elevated in the first and second trimester (Huisman and Aarnoudse, 1986; Murphy et al., 1986; Lu et al., 1991). In pregnancy-induced hypertension, plasma volume seems to become contracted in midpregnancy, after an initially normal increase in the first trimester (Blekta et al., 1970; Gallery et al., 1979), but well before the development of the symptomatology typical of the hypertensive disorder. The observation that total extracellular fluid volume had not changed in preeclamptics (Brown et al., 1989; Brown et al., 1992b) supports an altered distribution of extracellular fluid between the intra - and extravascular compartments, possibly secondary to the wellknown excessive rise in capillary permeability in these pregnancies. Whether pregnancies complicated by fetal growth retardation represent a similar syndrome, but without systemic maternal symptomatology, is still unsettled. In pregnancies complicated by fetal growth retardation, superimposed preeclampsia leads to an extra compromise of the already subnormal plasma volume and stroke volume (Arias 1975; Hays et al., 1985a; Nisell et al., 1988).

\subsection{Control of extracellular fluid volume}

The extracellular water is distributed over the intravascular (plasma) and extravascular compartments (interstitium). Despite large variations in dietary salt and water intake, the total amount of extracellular fluid, as well as its distribution over the two compartments, is kept within narrow limits. The preservation of a constant extracellular compartment is achieved primarily by modulation of the renal sodium and water excretion. Various sensor and effector systems are involved in this regulation (Moe et al., 1991). Fluctuations in blood volume and associated changes in filling pressure provide the input signal for the volume regulatory system. This pressure signal is sensed by pressor receptors located in the large central veins and arteries, the heart, lungs, liver and central nervous system. The kidneys represent the effector site 
by modulating renal perfusion, glomerular filtration, and renal sodium and water excretion. The renal response is relatively slow. Therefore, rapid changes in blood volume have to employ alternative effector sites. A rapid increase in blood volume is achieved by inducing rapid hemodilution in response to nonosmotic ADH release (Schrier and Briner, 1991). Conversely, a rapid decrease in blood volume can be achieved by displacement of excess intravascular fluid to the interstitium, resulting in edema formation. The lack of a demonstrable response to the gradually increasing blood volume in pregnancy supports a resetting of the volume receptors (Leduc et al., 1991). Because of the latter, volume dissipation does not accelerate in response to the ongoing gestational volume expansion. Renal plasma flow and glomerular filtration rate (Dunlop 1980) are already markedly increased in early pregnancy. The associated salt losing tendency requires sodium retaining measures to be taken in order to keep the sodium balance positive for a prolonged period. Nevertheless, even in pregnancy, renal sodium handling is the most important determinant in the volume homeostasis and will be discussed in more detail in one of the following sections. The osmoregulatory system plays an important role in the volume homeostasis during the early first trimester (Davison et al., 1981b) and will therefore also be discussed separately.

\subsection{Changes in the mechanisms controlling sodium excretion}

Pregnancy requires the creation of a salt conserving state for a period of several months to allow the accumulation of ca. 8 litres of fluid. Sodium retention is needed to replenish the expanding maternal extracellular compartment and to meet the extra fluid demands of the growing conceptus (Baylis and Davison, 1990b). Nevertheless, several adjustments in pregnancy tend to enhance sodium wastage. Among these are the elevated glomerular filtration rate, the higher circulating levels of progesterone and natriuretic hormones and physical factors such as the decrease in plasma albumin and vascular resistance. A decrease in sodium excretion is promoted by the increased levels of aldosterone and other pregnancy-related hormones, increased renin activity and physical factors such as the influence of posture and increased ureteral pressure. The relative importance of these factors will be discussed in the subsequent sections.

\subsection{Glomerular filtration rate}

Several measurement techniques have been employed to estimate glomerular filtration rate in pregnancy. In addition to the complicated method of inulin clearance, and the most commonly used imprecise method of 24-hour creatinine clearance, the clearance of iohexol has been introduced as an alternative (Krutzen et al., 1992). Iohexol is a nonradioactive contrast medium which can be administered in one single dose. In a recent study in 10 pregnant subjects, the 24 -hours creatinine clearance 
was compared with that of inulin and with the indirect estimation of the glomerular filtration rate from the serum creatinine (Davison and Hytten, 1974). The 24-hours creatinine clearance not only yielded consistently lower estimates for glomerular filtration rate than did the other 2 methods, it also was associated with a coefficient of variation as high as $25 \%$ (Bröcher-Mortensen and Rödbro, 1976). Calculation of the glomerular filtration rate from the serum creatinine level does not improve the precision, but removes the error associated with urine collection. Moreover, in pregnancy this method is erroneous since body weight or size does not reflect kidney size (Davison 1987).

The glomerular filtration rate calculated from the 24-hour creatinine clearance displays a cyclic pattern during the menstrual cycle with ca. $20 \%$ higher values in the luteal phase (Davison et al., 1981b). In the first 8-10 weeks of pregnancy glomerular filtration increases to a level of $45 \%$ above that in the follicular phase of the menstrual cycle (De Alvarez and Bratvold, 1958; Sims and Krantz, 1958; Davison and Noble, 1981a; Davison et al., 1981b). This increase is maintained for the remainder of pregnancy (Davison and Hytten, 1974; Dunlop 1980; Whichman and Rydén, 1986; Krutzen et al., 1992). A presumed decline in the final weeks of pregnancy (Davison 1987) is difficult to confirm because of the late-pregnancy increase in measurement error. In that period the measurement result is strongly influenced by the subject's position during measurement (Pritchard et al., 1955; Chesley and Sloan, 1964; Lindheimer et al., 1969). During pregnancy, the circadian rhythm in creatinine output, present in the nonpregnant state, is maintained provided that measurements are performed in the lateral position (Kalousek et al., 1969).

Glomerular filtration rate is determined by three factors: 1. the hydrostatic and oncotic pressure gradients across the glomerular membrane, 2 . the glomerular plasma flow and 3. the glomerular permeability (Moe et al., 1991). Only the first two factors are known to decrease in early pregnancy. A lower hydrostatic pressure can be expected to decrease, and a lower oncotic pressure to increase the glomerular filtration rate. However, their combined effect on the glomerular filtration rate in early pregnancy is obscure. The lack of an ongoing increase in glomerular filtration rate after the first trimester while serum albumin continues to decrease suggests that the pregnancy-related rise in glomerular filtration rate develops independent of changes in oncotic pressure (Baylis et al., 1977). In animal studies, the gestational rise in renal plasma flow is entirely explained by the concomitant rise in single-nephron glomerular filtration rate (Baylis 1980; Canton et al., 1982). A similar mechanism may also be operative in human pregnancy, as the effective renal plasma flow increases both in concert with the rise in glomerular filtration rate, and by a larger fraction (Sims and Krantz, 1958; Dunlop 1980). Because of the latter, the concomitant filtration fraction decreases in early pregnancy (Deen et al., 1972; Dunlop 1980). Two findings suggest that progesterone may be involved in this adaptation. Firstly, progesterone has been found to trigger a comparable set of renal changes in healthy volunteers (Oparil et al., 1975). Secondly, the possible reversal of the adaptive changes in renal function in the near-term period coincide with a decline in progesterone (Davison and Dunlop, 1980; Dunlop 1980). Nevertheless, disclosure of the exact mechanism responsible for the early-pregnancy adaptation in renal function would require serial measurement of effective renal plasma 
flow together with glomerular filtration rate from the time of implantation until the 8th week.

The fractional salt excretion is reduced in early pregnancy (Davison 1984a; Gallery and Brown, 1987), probably to compensate for the elevated glomerular sodium filtration. The enormous renal capacity for conserving sodium is particularly obvious during pregnancy, when a positive sodium balance of 2-6 mmol per day is achieved in spite of a 50\% higher glomerular sodium filtration (Gallery and Brown, 1987; Lindheimer and Katz, 1991b). An interesting observation in this respect is that, in the rat sodium retention is not essential for the plasma volume expansion in early pregnancy (Baylis and Munger, 1990a). Pregnant rats subjected to complete salt deprivation did not appear to have any appreciable difficulty in expanding their plasma volume in the first 10 days of pregnancy. Their plasma volume probably increases because of a redistribution of extracellular fluid in favor of the vascular bed and at the expense of the interstitium. This observation suggests that the extravascular fluid serves as a readily available fluid buffer that can be mobilized to compensate for a fall in vascular filling, in anticipation of the (slow) volume expansion by renal salt retention.

The mechanism reponsible for the rise in effective renal plasma flow and glomerular filtration rate is largely unknown. The role of the trophoblast is not essential, as similar changes have also been observed in pseudopregnancy (Atherton et al., 1982; Baylis 1982). Until recently, the gestational changes in the kidney were considered to develop in response to a primary rise in blood volume. Plasma volume expansion in rats failed to mimick the altered glomerular hemodynamics seen in pregnancy (Reckelhoff et al., 1989). Nowadays, it is more commonly accepted that the increase in glomerular filtration precedes that in blood volume and represents the renal expression of a primary generalized vasodilation (Davison 1987). The underlying mechanism of this vasodilatation is still obscure, but there is increasing evidence that a number of peptide regulatory factors (cytokines, binding proteins, growth factors) released in an appropriate steroid environment play an integral part in its mediation (Daunter 1992; Fay and Grudzinskas, 1991; Vinatier et al., 1992). Postpartum, the glomerular filtration rate remains elevated for at least one week and returns to the prepregnant level within one month (Chesley et al., 1959; Krutzen et al., 1992). The postpartum levels of glomerular filtration rate are comparable to those obtained preconceptionally (Davison and Dunlop, 1980).

In preeclampsia, glomerular filtration rate and renal plasma flow may decrease by as much as $25 \%$ to $35 \%$, in concert with the development of manifest clinical symptomatology. The decrease follows after an initial normal increase. The compromised renal function is accompanied by typical characteristic lesions of glomeruloendotheliosis (Lindheimer and Katz, 1991b).

\subsection{Natriuretic hormones}

In the course of pregnancy, plasma progesterone increases to levels more than 20 -fold higher than the luteal average. The natriuretic effect of this hormone is asso- 
ciated with its antimineralocorticoid effect, as well as its inhibitory effect on proximal tubular reabsorption (Oparil et al., 1975; Baylis and Davison, 1990b). The role in pregnancy of various other endocrine and paracrine substances with natriuretic potency, such as arginine vasopressine, circulating neurophysins, melanocyte stimulating hormone and vasodilating prostaglandins is still unsettled (Davison 1984a).

In recent years, alpha atrial natriuretic factor or peptide ( $\alpha$-ANP) has been suggested to be important for the volume homeostasis during pregnancy. This peptide is released by the atrial endothelial lining in response to increased atrial wall stretch or pressure (Brenner et al., 1990, Struthers 1994). $\alpha$-ANP reduces systemic blood pressure directly by inducing vascular relaxation and indirectly by inhibiting the reninangiotensin system. In pregnancy, the latter effect may be opposed by the increased progesterone levels, which promotes fluid expansion (Mulay et al., 1993). ANP also reduces the intravascular volume by enhancing renal natriuresis (Lowe et al., 1992a) and stimulating transudation toward the interstitium (De Vries et al., 1990). During the menstrual cycle $\alpha$-ANP is lower in the luteal phase (Jensen et al., 1989; Hussain et al., 1990). In early rat (Castor et al., 1989) and baboon pregnancy (Phippard et al., 1988), $\alpha$-ANP levels were lower than before pregnancy (Fournier et al., 1991). Results regarding the pattern of $\alpha$-ANP in human pregnancy have been conflicting, most likely because of one or a combination of the following factors: cross-sectional study design, heterogeneity of the study population, methodological factors such as lack of standardization of blood sampling (Lowe et al., 1991a) and blood sample processing. Critical analysis of the large body of data reported on this subject (Rutherford et al., 1987; Steegers et al., 1987; Hirai et al., 1988; Milsom et al., 1988; Thomsen et al., 1988; Bond et al., 1989; Vytiska-Binstorfer et al., 1989a; Vytiska-Binshofer et al., 1989b; McCance et al., 1990; Steegers 1990; Finn et al., 1991; Steegers et al., 1991; Hatjis et al., 1992; Lowe et al., 1992b; Thomsen et al., 1993) suggests little change in $\alpha$-ANP during human pregnancy, except for a possible slight transient fall in early pregnancy (Stratta et al., 1988). During most of twin pregnancy ANP levels are lower than during singleton pregnancies (Thomsen et al., 1994).

Postpartum $\alpha$-ANP levels showed a steep increase during the first days after delivery in some studies (Rutherford et al., 1987; Steegers et al., 1987; Duprez et al., 1989; Hatjis et al., 1992) but no increase in other studies (Bond et al., 1989; VytiskaBinstorfer et al., 1989a). M-mode echocardiography revealed that at 24 and 48 hours postpartum left atrial diameters were larger than in late pregnancy, due to the increased preload (Robson et al., 1987). A possible concomitant increase in atrial pressure could explain the rise in ANP levels in this period. After one week, ANP levels had returned to prepregnancy values (Steegers 1990). Others have reported ANP levels to be still increased, relative to a control group, more than one year after delivery (Finn et al., 1991). In the latter study it was suggested that pregnancy may have a protracted effect on volume regulation.

Most studies on $\alpha$-ANP in preeclamptic pregnancies report an increase (Thomsen et al., 1987; Visser et al., 1987; Hirai et al., 1988; Bond et al., 1989; Hatjis et al., 1989; Vytiska-Binstorfer et al., 1989b; Lowe et al., 1991b; Grunewald et al., 1994). However, the mechanism responsible for this increase is still obscure (Steegers 1990). It is unlikely that the endothelial cells of the atrial wall release an abnormally large 
amount of $\alpha$-ANP in response to the stimulus of atrial wall stretch or pressure. This isevidenced by the fact that the percentage of $\alpha$-ANP rise to volume loading is comparable to that in uncomplicated pregnancies (Nisell et al., 1992). Therefore, the elevated $\alpha$-ANP levels in preeclamptic pregnancies are either due to an abnormal pressure/stretch profile at the production site, such as may prevail in a chronic state of venoconstriction (Schrier and Briner, 1991), or to a reduced metabolic clearance rate by the kidneys. Since extravasation of albumin (De Vries et al., 1990) and capillary permeability (Poulsen et al., 1993) is enhanced by $\alpha$-ANP, this hormone may contribute to worsen the hypovolemia in preeclamptic pregnancies (Fournier et al., 1991). The pattern of $\alpha$-ANP during pregnancies complicated by impaired fetal growth has been reported in one study during third trimester (Salas et al., 1993). In this study ANP levels were not different from those in women with normal size fetuses. It is still not known if the recently discovered brain or B-type natriuretic peptide and especially C-type natriuretic peptide, which is stored in vascular endothelial cells, plays a role in the maternal adaptation to pregnancy (Struthers 1994).

\subsection{Antinatriuretic hormones}

Angiotensin II, the end product of the renin-angiotensin system, varies as a function of diastolic blood pressure in normotensive pregnancies (Symonds and Broughton Pipkin, 1978). It also has a direct effect on renal function by accelerating tubular sodium reabsorption and influencing renal hemodynamics (Hollenberg 1984). During pregnancy, the peripheral concentrations of both precursors and hormones involved in the renin-angiotensin system are markedly elevated. This rise may be, at least in part, related to the marked change in the steroid environment. This is suggested by the cyclic pattern in these precursors and hormones during the normal menstrual cycle, with most of them peaking during or shortly after ovulation (Brown et al., 1964; Sealey et al., 1987). During pregnancy the changes are as follows. Total renin concentration, subdivided into a bound (inactive) and free (active) fraction, decreases after an initial rise in the first trimester. Meanwhile, the concentration of active renin in the peripheral blood continues to increase until the 30th week (Weir et al., 1971; Skinner et al., 1972; Oats et al., 1981), in concert with angiotensinogen and angiotensin II (Weir et al., 1975). Afterwards, active plasma renin declines gradually until term (Ledoux et al., 1975; Oats et al., 1981; Karlberg et al., 1984). During labor angiotensin II increases in concert with diastolic blood pressure (Symonds and Broughton Pipkin, 1980). Within the first week after labor, active renin, angiotensinogen and angiotensin II return to their prepregnancy levels (Broughton Pipkin et al., 1978; Jadoul et al., 1982; Steegers 1990). In spite of the higher basal activity of the renin-angiotensin system during pregnancy, its response to external stimuli remains unaffected (Brown et al., 1987; Broughton Pipkin 1988). Apparently, pregnancy induces a resetting of this system, comparable to that of the baroreceptor (Leduc et al., 1991) and osmoregulatory systems (Davison et al., 1981b). The effect of the resetting consists of a lower threshold of serum sodium levels for the activation of the renin-angiotensin system (Gallery and Brown, 1987). On the other hand, neither the mechanism responsible for the resetting nor the interrelation between the changes in the renin-angiotensin system and volume 
homeostasis during pregnancy have been elucidated. Data suggesting a role for estrogen (Longo 1983; Siimes et al., 1983) and vasodilating prostaglandins (Friedman 1988 ) in the changes in the renin-angiotensin system are inconclusive.

The renin-angiotensin system regulates the aldosterone release. Aldosterone concentration as well as turnover rate are raised in pregnancy to levels as high as those in primary aldosteronism (Lindheimer and Katz, 1991b). However, even in the first trimester the strong aldosterone dependence of renin which prevails in the nonpregnant state begins to weaken. This is suggested by the increase in aldosterone preceding that in renin (Brown et al., 1988a). The suggested superimposed effect of the increasing progesterone levels has never been substantiated (Ledoux et al., 1975; Smeaton et al., 1977; Brown et al., 1986a). It is conceivable that the rapid rise in effective renal plasma flow facilitates the release of aldosterone in the kidneys (Weir et al., 1975; Brown et al., 1986a). Other potential contributors are the abruptly developing hemodilution (Davison et al., 1981b) and the change in steroid environment. Although the preserved response of aldosterone to changes in serum sodium and vascular filling supports a simple reset to a lower sodium setpoint, it does not exclude that part of the hyperaldosteroneism in pregnancy is caused by suboptimal expansion of the extracellular volume (Baylis and Davison, 1990b).

Several other hormones may enhance sodium retention during pregnancy. Among them is desoxycorticosterone one of the most potent mineralocorticoids, which shows a steady increment during pregnancy, especially during the third trimester (Nolten et al., 1979). The pattern of change in this hormone resembles those of progesterone, aldosterone and estrogen. Most of the desoxycorticosterone is bound to carrier proteins, which also increase with advancing pregnancy. Obviously, protein binding diminishes the biological activity of desoxycorticosterone. The role of vasodilating prostaglandins, estrogens, prolactin, placental lactogen, growth hormone, ACTH and cortisol with respect to their potential antinatriuretic action in pregnancy is still poorly understood (Baylis and Davison, 1990b).

In mild preeclampsia, both plasma renin activity and angiotensin II were found to be either unchanged or slightly increased, whereas in severe preeclampsia they had both decreased (Fievet et al., 1985; August et al., 1990; Seeley et al., 1992) together with aldosterone (Weir et al., 1973; Karlberg et al., 1984; Fievet et al., 1985; Pedersen et al., 1985; August et al., 1990). Consequently, the different response of the renin-angiotensin system in preeclamptic subjects is likely to represent only a secondary effect, resembling that previously outlined for the higher $\alpha$-ANP levels. The concomitantly contracted plasma volume in this condition (Gallery et al., 1979) suggests either an excessive central vascular stimulus or abnormal processing of normally triggered reflexes (e.g. defective receptor binding or inadequate renal response). At any rate, the renin-angiotensin system seems to play only a secondary role in the pathogenesis of severe preeclampsia (Broughton Pipkin 1988). The mechanisms involved in the development of hypertensive disorders of pregnancy have been elaborated recently in two extensive reviews (Gallery and Brown, 1987; Broughton Pipkin 1988). 


\subsection{The osmoregulatory system}

The plasma osmolality in the nonpregnant state is maintained within narrow limits. Nevertheless, it shows a cyclic pattern during the menstrual cycle with values reduced by about $5 \mathrm{mosmol} / \mathrm{l}$ in the luteal phase (Vokes et al., 1988). About 3 to 4 weeks after conception the osmolility decreases by $8-10 \mathrm{mosmol} / \mathrm{kg}$ (Davison et al., 1981b), and continues to decrease slightly in the subsequent 4 weeks. After the 10 th week the osmolality changes little in the remainder of pregnancy. The decrease is primarily caused by a fall in plasma sodium concentration, with a modest contribution of the lower plasma urea concentration. In the nonpregnant state an appreciable decrease in osmolality such as that in pregnancy would lead to interruption of the release of antidiuretic hormone, thus inducing water diuresis. Because of osmoreceptor resetting without change in receptor sensitivity in early pregnancy, the normal response (vasopressin release) to variable water intake is maintained at a lower osmolality treshold. This resetting has been studied in human and rat pregnancy (Davison et al., 1984b; Barron and Lindheimer, 1988; Davison et al., 1988). Both physiological meaning and triggering mechanism are still obscure. Experiments in rats, aimed at reproducing the gestational decrease in osmolality by the administration of a variety of pregnancy hormones or by inducing a state of pseudopregnancy have been unsuccessful (Barron et al., 1986; Barron and Lindheimer, 1988). This suggests that the presence of the fetoplacental unit is essential for the gestational osmoresetting. In nonpregnant women, human chorionic gonadotrophin injections were found to decrease their osmotic threshold by $5 \mathrm{mosmol} / \mathrm{kg}$ (Davison et al., 1988). The concomitant expansion of both size and content of the vascular compartment in pregnancy may be associated with a change in the sensitivity or response of the volume receptors in the heart and large veins. Hypotension and hypovolemia are the most important nonosmotic stimuli for vasopressin release (Baylis 1987). Whether the early-pregnancy fall in blood pressure and vascular filling state trigger the osmoreceptor resetting has never been satisfactorily demonstrated (Schrier and Briner, 1991). On the other hand, neither an imposed hypervolemia nor a (pharmacologically) imposed hypotension could alter the normal gestational resetting of the plasma osmolality in the rat (Barron et al., 1989).

The gestational changes in the osmoregulatory system are paralelled by a progressively increasing vasopressin metabolization in pregnancy (Davison et al., 1989), with only little effect on circulating vasopressin levels (Davison et al., 1984b; Brown et al., 1988a). The rapidly increasing vasopressin turnover rate is caused by the action of a local placental factor (Landon et al., 1988) together with a progressively increasing activity of placental vasopressinase (Davison et al., 1989).

Plasma osmolality and vasopressin do not change appreciably in preeclamptic pregnancies despite plasma volume contraction (DeVane 1985; Pedersen et al., 1985; Brown et al., 1986b). This observation suggests a modest role of the osmoregulatory system in the pathogenesis of hypertensive disorders of pregnancy. 


\subsection{Conclusions}

Maternal hemodynamic adaptation to pregnancy consists of profound changes in various interdependent systems. Of crucial importance in the early adaptation of the volume homeostatic mechanisms to pregnancy is the resetting of the volume and osmoreceptors. This resetting may be induced by a reduction in vascular tone and leads to early changes in plasma osmolality and glomerular filtration rate. After this initial adaptation other volume-regulating mechanisms such as the renin-angiotensinaldosterone system, pregnancy hormones, and $\alpha$-ANP adapt to the rising blood volume. The initial adaptation results in a state of relative vascular underfill, inducing secondary compensations in the volume homeostasis. The initially increased vascular capacitance in pregnancy is compatible with the signals of vascular overfill. Contrarily, the protracted filling of this enlarged vascular bed triggers signals compatible with vascular underfill (Schrier and Dürr, 1987). 
Chapter 4

\section{MEASUREMENT OF ANTERIOR-POSTERIOR DIAMETER OF INFERIOR VENA CAVA BY ULTRASONOGRAPHY: A NEW NON-INVASIVE METHOD TO ASSESS ACUTE CHANGES IN VASCULAR FILLING STATE}

Johannes J Duvekot MD ${ }^{1}$, Emile C Cheriex $\mathrm{MD}^{2}$, Wie Djin Tan MD ${ }^{3}$, Guido AK Heidendal MD, $\mathrm{PhD}^{3}$, and Louis LH Peeters MD, $\mathrm{PhD}^{1}$

From the Departments of Obstetrics and Gynecology ${ }^{1}$, Cardiology ${ }^{2}$ and Nuclear Medicines, University Hospital Maastricht, PO Box 1918, 6214 BX Maastricht, The Netherlands.

Cardiovascular Research 1994; 28: 1269-1272. 


\subsection{Abstract}

Objective:

Methods:

Results:

\section{Conclusions:}

The aim was to determine whether measurement of the anterior-posterior diameter of the inferior vena cava by ultrasonography, before and during deep inspiration, allows indirect information on baseline blood volume and changes in vascular filling state to be obtained in healthy subjects.

Blood volume was measured in 12 volunteers by a standard radioactive isotope method. The inferior vena cava dimensions were measured by ultrasound in various positions. In addition, in 10 male subjects the effect on the inferior vena cava dimensions of volume loading was evaluated by rapid infusion of plasma expander. During and immediately following volume loading, the inferior vena cava dimensions and blood volume were remeasured.

Neither the baseline inferior vena cava diameters nor any combination of these parameters correlated with the concomitant blood volume. Volume loading increased the anterior-posterior inferior vena cava dimensions linearly, the largest increase being observed with the maximal inferior vena cava diameter and the subject in supine position.

It follows that ultrasonic inferior vena cava indices cannot be used as accurate indirect estimate of baseline blood volume, but provide reliable information on acute changes of the subjects' vascular filling state. 


\subsection{Introduction}

Normal cardiovascular function requires maintenance of circulating blood volume and vascular filling state within narrow limits. However, it is still uncertain how changes in volume homeostasis take place in a condition of chronically increased circulatory function, such as prevails during fever, exercise and pregnancy. In order to increase our understanding of functional changes in blood volume and vascular filling, an easy, non-invasive, reliable and repeatedly applicable measurement technique is necessary as an alternative to the methods which require the use of radioactive tracers or vital dyes. In a clinical setting the inferior vena cava diameter measured by ultrasound, before and during deep inspiration, has been shown to provide useful information on right atrial pressure, "dry weight" and baseline blood volume in patients undergoing hemodialysis (Cheriex et al., 1989). Whether this technique can also generate valuable information on blood volume and changes in vascular filling state in healthy subjects, has not yet been explored.

The present study was designed to test the hypothesis that the ultrasonic inferior vena cava diameter provides valuable information on both baseline blood volume and imposed rapid changes in vascular filling state in a population of healthy subjects. To this end, we evaluated: 1 . The relationship between the baseline ultrasonic inferior vena cava diameter measured before and during deep inspiration, and blood volume, the latter being measured simultaneously by a standard radioactive isotope technique and 2. the quantitative effect on the ultrasonic inferior vena cava dimensions of stepwise increments in vascular filling state induced by plasma volume expansion with the plasma expander polygeline (Haemaccel $\left.{ }^{\mathrm{R}}\right)$.

\subsection{Subjects and methods}

Selection and characteristics of subjects: The study protocol was approved by the medical ethics committee of the Academic Hospital of Maastricht. After providing detailed explanation about the protocol and following informed consent, 10 male and six female subjects were enrolled in this study. All subjects were healthy, normotensive and ranged in age from 25 to 33 years. Body weight ranged from 54.5 to $103.5 \mathrm{~kg}$, and body surface area from 1.60 to $2.42 \mathrm{~m}^{2}$ (Dubois and Dubois, 1916). The wide interval in weight was almost entirely related to the wide range in body length as none of the subjects deviated by more than $1 \mathrm{SD}$ from their ideal weight (Feldschuh and Enson, 1977). All measurements were performed between 1500 and $1800 \mathrm{~h}$. Prior to the study, accessibility of the inferior vena cava for ultrasonic measurements was evaluated and absence of cardiovascular pathology was demonstrated by echocardiography and electrocardiography. In the first six consecutive males and all six females, plasma volume was measured according to the radioiodinated serum albumin method (Keyes et al., 1973). After this procedure volume loading was performed in the male subjects only, by infusing $1000 \mathrm{ml}$ of polygeline. In these six male subjects, the dilution of the initially injected ${ }^{125} \mathrm{I}$-albumin, $10 \mathrm{~min}$ after volume loading, indicated that the loss of the plasma expander from the intravascular space in 
this time span was negligible. The plasma volume measurement with ${ }^{125} \mathrm{I}$-albumin was therefore omitted from the protocol for the subsequent four male volunteers in this study. The volume loading experiment was not performed in the female subjects, because of likely influences of cyclic hormonal changes on blood volume homeostasis and venous tone (Hassan et al., 1990).

Echographic and laboratory measurements: The methodology of plasma volume measurement was as follows. Five $\mu \mathrm{Ci}{ }^{125} \mathrm{I}$ human albumin, dissolved in $1 \mathrm{ml}$ saline, was given intravenously (total body dose of irradiation $=0.04 \mathrm{mSv}$ ). After 10 , 20 and $30 \mathrm{~min}$, blood samples $(5 \mathrm{ml})$ were withdrawn to determine the packed cell volume (vol \%) and the volume specific plasma radioactivity $(\mathrm{mCi} / \mathrm{ml})$. The virtual volume specific plasma radioactivity at the time of bolus injection, was calculated by extrapolation using the three serially obtained volume specific plasma radioactivity data points. Plasma volume was calculated by dividing the total injected radioactivity by the virtual volume specific plasma radioactivity at time zero, which is the time of bolus injection. Blood volume was calculated assuming unchanged blood cell volume, by multiplying plasma volume with (1/1-packed cell volume). After the last sampling, $1000 \mathrm{ml}$ of the plasma expander polygeline was infused intravenously within $15 \mathrm{~min}$ at a constant rate of $\approx 70 \mathrm{ml}$. $\mathrm{min}^{-1}$. Ten, 20 and $30 \mathrm{~min}$ after volume loading, a second set of blood samples was collected. Plasma volume was calculated after volume loading by dividing the virtual volume specific plasma radioactivity by that in each of these three samples.

Images of the inferior vena cava were made from the subxyphoidal region (Cheriex et al., 1989). In the segment between 5 and $30 \mathrm{~mm}$ below the right atrium, the inferior vena cava measurement is most responsive to the respiratory excursions (Simonson and Schiller, 1988). On the other hand, inferior vena cava becomes smaller and thus measurement error larger with increasing distance from the heart (Nakao et al., 1987). Therefore, long and short axis views of the inferior vena cava were obtained just below the diaphragm in the hepatic segment. Echographic images were produced using a $3.5 \mathrm{MHz}$ phased-array transducer (Hewlett-Packard Sonos 1000). M-mode echograms were recorded at a paper speed of $25 \mathrm{~mm} . \mathrm{s}^{-1}$. M-mode echogram and electrocardiogram were recorded simultaneously. The maximal antero-posterior inferior vena cava diameter (inferior vena cava diameter maximal $_{\text {) was measured }}$ during unforced expiration and before the P-wave on the ECG to avoid interference with the A wave of the venous pressure curve. Maximum depletion of the abdominal inferior vena cava which leads to the smallest inferior vena cava contour (inferior

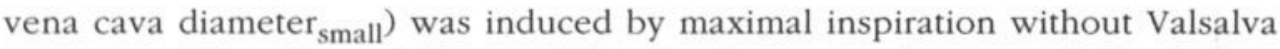
manoeuvre. The collapse index was defined as the percent decrease with inspiration: (inferior vena cava diameter maximal $_{\text {- inferior vena cava diameter }}$ small $_{\text {) }}$ inferior vena cava diameter maximal $\times 100 \%$. The part of the inferior vena cava that collapses during

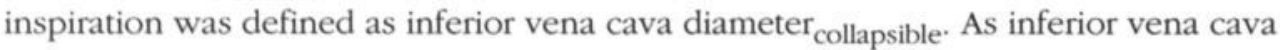
diameter $_{\text {collapsible }}$ always represents the outermost layer of the more or less circular inferior vena cava, the absolute value for inferior vena cava diameter collapsible $_{\text {at a given }}$ collapse index increases as a function of inferior vena cava diameter maximal. This phenomenon was corrected for by redefining inferior vena cava diameter collapsible $_{\text {as the }}$ square root of the difference between the squared inferior vena cava diameter maximal $_{\text {. }}$. 


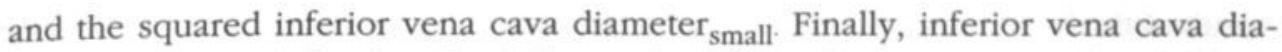
meter $_{\text {minimal }}$ was defined as the difference between inferior vena cava diameter maxi- $_{\text {- }}$ $\mathrm{mal}$ and inferior vena cava diameter ${ }_{\text {collapsible }}$

Ultrasonic measurement of the inferior vena cava dimensions was performed before, immediately after 500 and $750 \mathrm{ml}$ volume loading, and $10 \mathrm{~min}$ after volume loading, with the subject in supine position. In the first six consecutive subjects measurements were also performed antero-posteriorly from the long axis views in left and right lateral position. Finally, possible diurnal variation in the inferior vena cava indices was evaluated by subjecting all male subjects to a second measurement session in the early morning, at least one week after the first measurement. All echographic studies were performed by one of the coauthors (ECC).

Statistical methods: Baseline data obtained in subjects who underwent plasma volume determinations were compared using the Mann-Whitney U test. As none of the inferior vena cava indices measured differed between males and females, the 12 data points were pooled. The correlation of the various inferior vena cava dimensions with baseline plasma volume and blood volume, indexed for either weight or body surface area, was evaluated by Spearman correlation analysis and linear regression. The change in the inferior vena cava dimensions in response to a stepwise increase in vascular filling state up to $1000 \mathrm{ml}$ was evaluated by both paired Student $t$ test and linear regression, using the imposed volume load as the independent variable. A probability ( $p$ value) of less than $5 \%$ was considered significant. All data are presented as means (SD) throughout the text, unless otherwise stated.

\subsection{Results}

Mean packed cell volume, plasma volume and blood volume for the male and female subjects are listed in Table I.

Table I Mean (SD) values for body surface area $\left(\mathrm{m}^{2}\right)$, packed cell volume (vol \%), blood volume ( $\mathrm{ml}$ ), and plasma volume $(\mathrm{ml})$ in six male and six female subjects. Plasma volume was measured by the radioiodinated serum albumin metbod.

\begin{tabular}{llll}
\hline & All & Male & Female \\
\hline body surface area & $1.91 \pm 0.21$ & $2.07 \pm 0.17$ & $1.74 \pm 0.08^{\circ}$ \\
packed cell volume & $40 \pm 4$ & $42 \pm 3$ & $38 \pm 3^{\circ}$ \\
blood volume & $5391 \pm 980$ & $6159 \pm 760$ & $4623 \pm 348^{\circ}$ \\
blood volume $/ \mathrm{m}^{2}$ & $2810 \pm 242$ & $2968 \pm 182$ & $2653 \pm 190^{\circ}$ \\
plasma volume & $3158 \pm 454$ & $3536 \pm 308$ & $2780 \pm 128^{\circ}$ \\
plasma volume $/ \mathrm{m}^{2}$ & $1652 \pm 103$ & $1709 \pm 100$ & $1596 \pm 76$ \\
\hline
\end{tabular}

$\cdot p<0.05$ v male (Mann-Wbitney $U$ test).

Blood volume per square metre was lower $(\mathrm{p}<0.05)$ in women than in men, at 2653 (SD 190) v 2969 (SD 183). The difference in plasma volume/ $\mathrm{m}^{2} \mathrm{did}$ not reach significance $(\mathrm{p}=0.054)$, indicating that the difference in blood volume $/ \mathrm{m}^{2}$ was mostly a 
result of the higher packed cell volume in the male subjects. By Spearman regression of the 12 data points combined, none of the baseline inferior vena cava dimensions, whether evaluated alone or in any combination, correlated with baseline blood volume or plasma volume, irrespective of whether the latter two variables were expressed per unit body surface area or per unit weight. For example, the Spearman correlation coefficients for the relation between blood volume and inferior vena cava indices ranged from -0.10 (inferior vena cava diameter minimal $_{1}, \mathrm{p}=0.37$ ), -0.31 (inferior vena cava diameter collapsible, $_{\text {p }}=0.16$ ) to -0.33 (inferior vena cava diameter maximal. $\mathrm{p}$ $=0.15$ ) (fig 1).

In the first six consecutive male volunteers, volume loading increased the mean measured plasma volume by $986 \mathrm{ml}$ (range: $779-1301 \mathrm{ml}$ ). Volume loading was performed in a total of 10 male subjects. In agreement with previous reports (Nakao et al., 1987; Rak et al., 1991), the ultrasonic inferior vena cava dimensions were found to be position-dependent with the largest baseline values seen in the right lateral position and the largest absolute changes observed in the supine position (Table II). We also noted a clear impact of the subjects' position on the shape of the inferior vena cava, round to oval in the left lateral and supine position and more slit-like shaped in the right lateral position.

Figure 1 Scattergram of the individual baseline values of inferior vena cava diameter maximal $_{\text {and }}$ blood volume $(n=12)$.

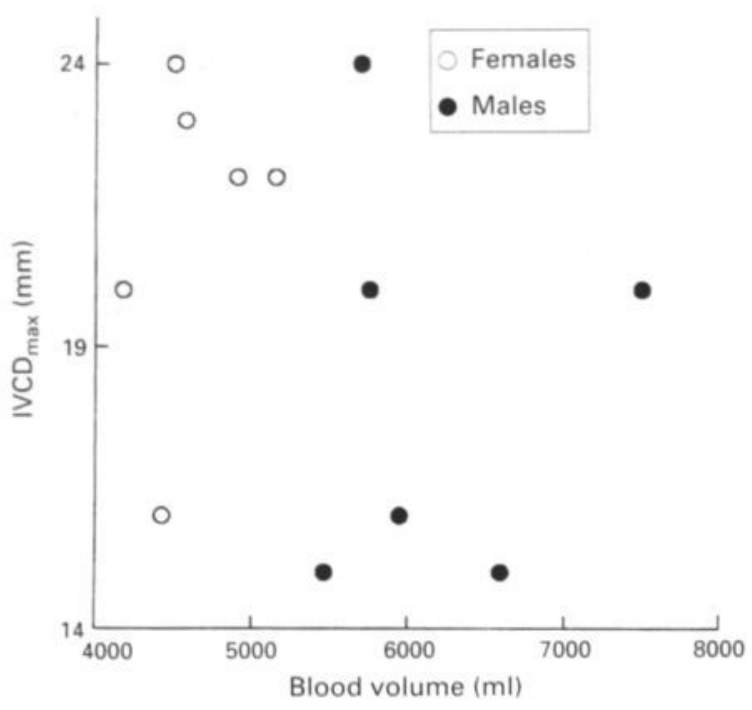

As the latter inferior vena cava shape led to an elevated coefficient of variation, particularly in the collapse index measurement (Table II), the right lateral position was considered unfavourable. On the basis of a higher absolute change in all inferior vena cava indices in the supine position, the change in these parameters was only evaluated with increasing vascular filling state (Table III). Inferior vena cava diameter $_{\text {maximal }}$ increased almost linearly (fig 2 ). 


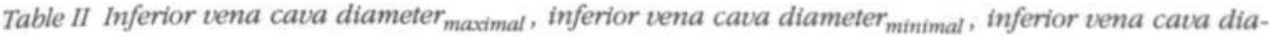
meter $_{\text {collapsible }}(\mathrm{mm})$, collapse index $(\%)$, and the induced absolute change in these variables, in supine, right lateral, and left lateral position before and after volume loading in the first six male subjects. Values are means (SD).

$\begin{array}{lll}\text { Baseline } & \begin{array}{l}\text { After volume } \\ \text { loading }\end{array} & \text { Change }\end{array}$

supine position:

IVCDmaximal

IVCD $_{\text {minimal }}$

IVCD collapsible

Collapse Index

right lateral position:

$\begin{array}{ll}\text { IVCD }_{\text {maximal }} & 24.7 \pm 3.8 \\ \text { IVCD }_{\text {minimal }} & 5.0 \pm 3.3 \\ \text { IVCD } & 19.7 \pm 4.0 \\ \text { Collapsible } & 45.0 \pm 20.3\end{array}$

left lateral position:
IVCD ${ }_{\text {maximal }}$
IVCD $_{\text {minimal }}$
IVCD collapsible

Collapse Index

$$
\begin{aligned}
& 18.3 \pm 3.3 \\
& 2.6 \pm 2.4 \\
& 15.8 \pm 1.9 \\
& 56.2 \pm 18.4
\end{aligned}
$$$$
24.3 \pm 4.3^{\cdots}
$$$$
+6.0
$$$$
9.0 \pm 7.4^{\circ}
$$$$
+6.4
$$$$
15.3 \pm 4.9
$$$$
-0.5
$$$$
30.1 \pm 18.3 *
$$

$26.8 \pm 3.2 *$

$8.4 \pm 3.3 \cdots$

$+3.4$

$18.5 \pm 3.8$

- 1.2

$29.1 \pm 11.2^{\circ}$

$-15.9$
$19.3 \pm 2.8 *$

$+4.8$

$7.8 \pm 5.9$

$+3.3$

$11.5 \pm 6.2$

$+1.5$

$29.8 \pm 22.4^{*}$
$-16.7$

IVCD = inferior vena cava diameter in antero-posterior plane.

${ }_{p}<0.05, \cdots p<0.01, \cdots p<0.001 v$ baseline (paired t test).

Figure 2 Inferior vena cava diameter ${ }_{\text {maximal }}$ during volume loading $(n=10)$. Error bars $=$ SEM. The solid line with (dashed) $95 \%$ confidence limits was calculated by linear regression.

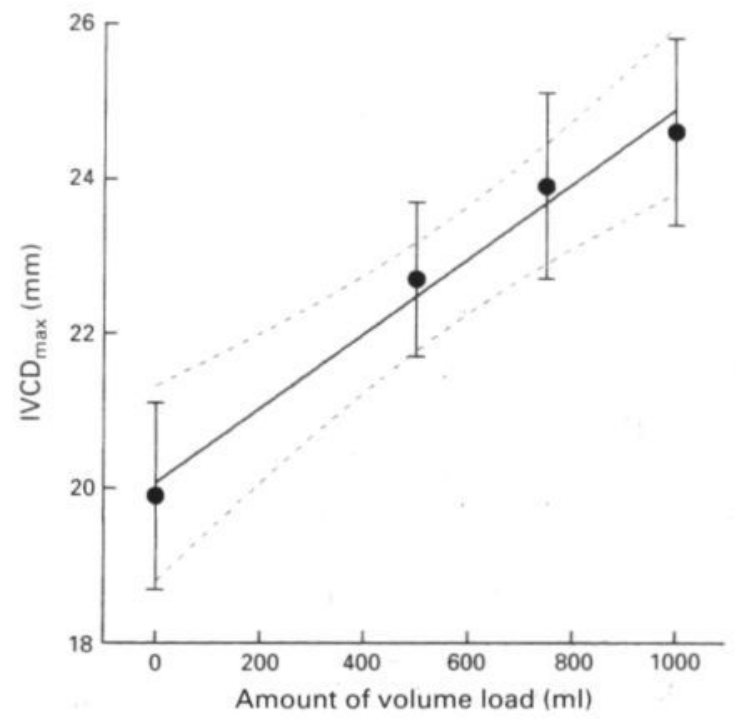


Table III Inferior vena cava diameter maximal, inferior vena cava diameter $_{\text {minimal, }}$ inferior vena cava diameter $_{\text {collapsible }}(\mathrm{mm})$ and collapse index (\%) in supine position before and after $500 \mathrm{ml}, 750 \mathrm{ml}$, and $1000 \mathrm{ml}$ of volume loading with polygeline in 10 male subjects. Values are means (SD).

\begin{tabular}{lllll}
\hline Volume loading & Before & After $500 \mathrm{ml}$ & After $750 \mathrm{ml}$ & After $1000 \mathrm{ml}$ \\
\hline IVCD $_{\text {maximal }}$ & $19.9 \pm 3.8$ & $22.7 \pm 3.3 *$ & $23.9 \pm 3.7 \cdots$ & $24.6 \pm 3.7^{* \cdots}$ \\
IVCD $_{\text {minimal }}$ & $2.7 \pm 1.9$ & $5.9 \pm 5.0^{*}$ & $6.6 \pm 5.3 *$ & $7.9 \pm 5.9^{*}$ \\
IVCD $_{\text {collapsible }}$ & $17.2 \pm 2.7$ & $16.8 \pm 4.0$ & $17.3 \pm 3.3$ & $16.7 \pm 4.3$ \\
Collapse Index & $54.2 \pm 14.5$ & $39.3 \pm 18.8 *$ & $37.1 \pm 17.0 *$ & $31.7 \pm 14.5 *$ \\
\hline
\end{tabular}

IVCD $=$ inferior vena cava diameter in antero-posterior plane.

$* p<0.05, \cdots p<0.01, \cdots p<0.001 v$ baseline (paired $t$ test).

Figure 3 Collapse index during volume loading $(n=10)$. Error bars $=$ SEM. The solid line witb (dashed) $95 \%$ confidence limits was calculated by linear regression.

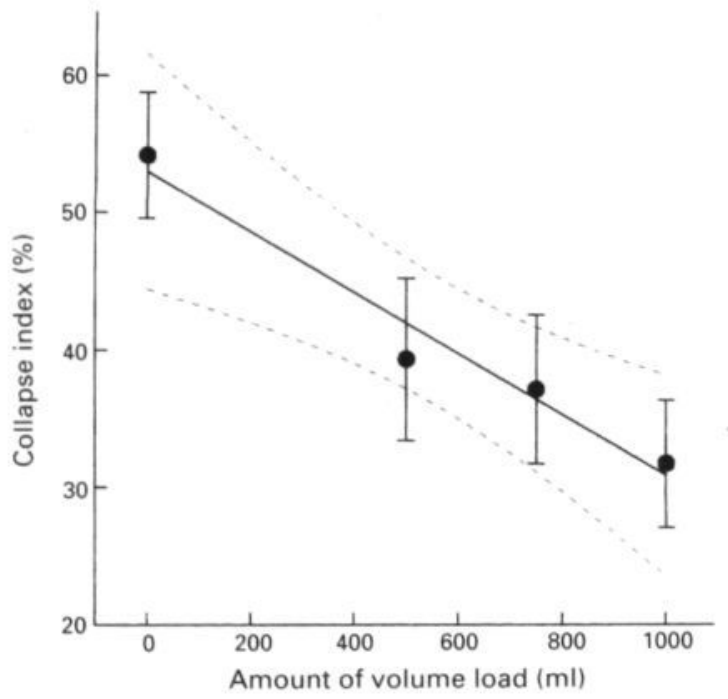

The rate of change, which did not differ between the 10 subjects in spite of widely variable baseline inferior vena cava diameter maximal $(14-28 \mathrm{~mm})$, amounted to $\approx 1.0$ $\mathrm{mm}$ per $200 \mathrm{ml}$ volume expansion.

The collapse index decreased consistently (Table III). However, the fall amounted to only $\approx 5 \%$ for each $200 \mathrm{ml}$ volume loading giving rise to a large measurement error (fig 3).

Evaluation of the partitioning of the change in inferior vena cava diameter maximal $_{\text {mation }}$ between inferior vena cava diameter ${ }_{\text {minimal }}$ and inferior vena cava diameter collapsible indicated that the increase in inferior vena cava diameter minimal $_{\text {during volume loa- }}$ ding accounted for the entire increase in inferior vena cava diameter maximal $_{\text {(Table III }}$ and fig 4). 
Figure 4 Inferior vena cava diameter minimal $_{\left(I V C D_{\text {minimal }}\right) \text { and inferior vena cava diameter }}$ collapsible $\left(I V C D_{\text {collapsible }}\right)$ during volume loading $(n=10)$. Error bars $=S E M$. The solid lines witb (dasbed) $95 \%$ confidence limits were calculated by linear regression.

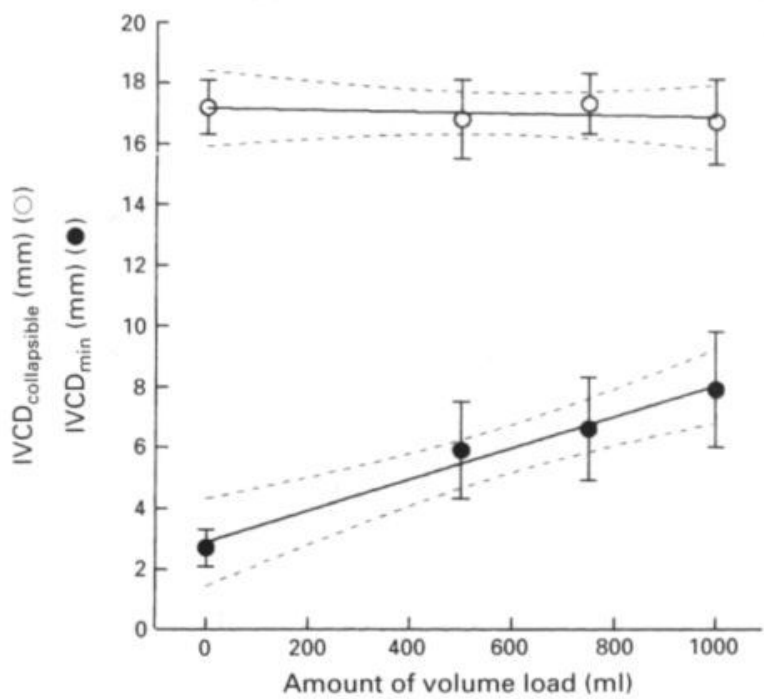

Interestingly, a comparable rate of change in inferior vena cava diameter minimal was $_{\text {a }}$ observed in all 10 subjects despite a wide variation in baseline inferior vena cava diameter $_{\text {minimal }}(0.3-6 \mathrm{~mm})$.

Finally, comparison of early morning with afternoon measurements suggested no appreciable diurnal variation in ultrasonic inferior vena cava dimensions, although all inferior vena cava indices tended to be smaller when measured in the morning (Table IV).

Table IV Inferior vena cava diameter ${ }_{\text {maximal }}$, inferior vena cava diameter ${ }_{\text {minimal }}$, inferior vena cava diameter collapsible $(\mathrm{mm})$ and collapse index $(\%)$ in supine position measured in morning and late afternoon in 10 male subjects. Values are means (SD).

\begin{tabular}{llll}
\hline & Morning & Afternoon & Significance \\
\hline $\mathrm{IVCD}_{\text {maximal }}$ & $19.2 \pm 2.0$ & $19.9 \pm 3.8$ & NS \\
$\mathrm{IVCD}_{\text {minimal }}$ & $1.6 \pm 1.2$ & $2.7 \pm 1.9$ & NS \\
$\mathrm{IVCD}_{\text {collapsible }}$ & $17.7 \pm 1.7$ & $17.2 \pm 2.7$ & NS \\
Collapse Index & $65.5 \pm 9.3$ & $54.2 \pm 14.5$ & NS \\
\hline
\end{tabular}

$I V C D=$ inferior vena cava diameter in antero-posterior plane, $N S=$ non significant difference morning $v$ afternoon (paired t test). 


\subsection{Discussion}

The objective of the present study was to determine whether the ultrasonic dimensions of the inferior vena cava and changes in these indices induced by volume loading, when measured under optimal and standardised conditions, could provide useful information about baseline blood volume and magnitude of changes in vascular filling state, respectively. Previous observations suggested that these indices could be measured with a surprisingly low degree of error (Cheriex et al., 1989): the interobserver variation in inferior vena cava dimensions and collapse index was less than $5 \%$ and $10 \%$, respectively, whereas the intraobserver variation was less than $2.5 \%$ for both indices.

The values found for the collapse index in our study did not significantly differ from the collapsability index or inspiratory collapse described by other investigators measured during normal inspiration (Moreno et al., 1984; Kirchner et al., 1990). We defined collapse index as the percentage of decrease in inferior vena cava diametermaximal after maximum inspiration without Valsalva manoeuvre. The most important advantage of our method of measuring collapse index is the lower intraindividual and interindividual variation (Cheriex et al., 1989). Deep inspiration accelerates venous return to a maximum plateau which corresponds to the plateau in the $\mathrm{S}$ shaped venous return curve (Guyton et al., 1986a). The negative intrathoracic pressure generated is passed on to the right atrium, thus causing rapid displacement of blood from the large central veins towards the heart (Natori et al., 1979). As a consequence, the intraluminal pressure in the inferior vena cava is likely to drop to zero. Therefore, the inferior vena cava diameter $r_{\text {minimal }}$ induced so can be expected to provide an indirect estimate of "unstressed volume", which is defined as the "static" fraction of blood volume needed for basal filling of the vascular bed (Rothe 1986). In contrast, the "stressed volume" forms the complement of the unstressed volume and represents the "dynamic" fraction of blood volume returned to the heart as "venous return"

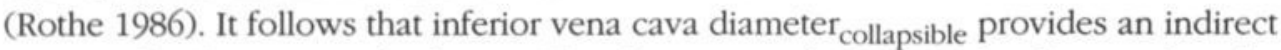
estimate for this "stressed volume". The observation that volume loading produced a

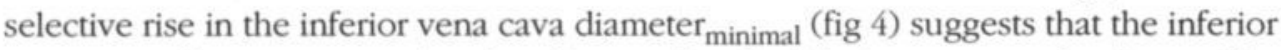
vena cava diameter ${ }_{\text {minimal }}$ is primarily correlated with the unstressed volume. The change in caval shape in response to deep inspiration from round to oval leads to a relatively large error in the measurement of the collapse index (Table III). This error is subsequently magnified in the calculation of the inferior vena cava diameter $r_{\text {minimal }}$

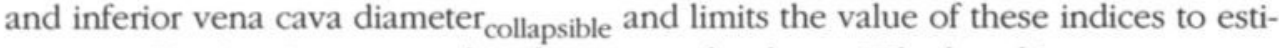
mate partitioning into stressed and unstressed volume. Whether this measurement error can be diminished by measuring inferior vena cava diameter area instead requires additional study.

In this study the inferior vena cava indices, separately or in various combinations, correlated poorly with baseline blood volume and plasma volume. This result may be partly due to error in the measurement of the "diameter" of a noncircular structure as was emphasized above. Also inferior vena cava diameter $r_{\text {maximal }}$ and its components inferior vena cava diameter ${ }_{\text {minimal }}$ and inferior vena cava diameter collap- $_{\text {- }}$ sible are possibly more dependent on other functional factors than blood volume (for 
example, intra-abdominal venous drainage, shunting, vascular wall characteristics, and regulatory setpoints) (Kerr et al., 1964; Moreno et al., 1973; Rothe 1983; Rak et al., 1991).

The second objective of the present study was to investigate the effect of a rapid rise in vascular filling state on the inferior vena cava indices. About $10 \mathrm{~min}$ after completion of volume loading, the measured plasma volume had increased by $\approx 1000 \mathrm{ml}$ which indicates that loss of infused fluid was negligible with the high infusion rate employed, an observation also reported by others (Davies 1987; Van de Broek et al., 1989). During volume loading the shape of the inferior vena cava did not change appreciably, an observation which agrees with that of a previous in vitro study (Moreno et al., 1973). The lack of consistent changes in mean arterial pressure, heart rate and cardiac output (Duvekot et al., 1994a) and the linearity of the rise, even between 750 and $1000 \mathrm{ml}$ (fig 1), indicated that the $1000 \mathrm{ml}$ of extra volume did not exceed the storage capacity of the large central veins (Guyton et al., 1973c; Rothe 1983). Therefore, the gradual increase in inferior vena cava diameter maximal $_{\text {and inferior }}$

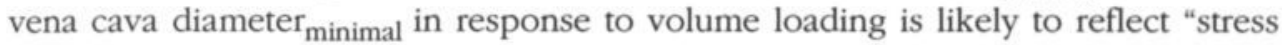
relaxation" of veins (Prather et al., 1969; Moreno et al., 1973). It is feasible that during volume loading right atrial pressure increased. In a similar experiment measuring right atrial pressure, an almost linear increase was found during volume expansion (Di Donato et al., 1982). This finding supports the theory that the inferior vena cava indices can also be useful in obtaining information on right atrial pressure (Moreno et al., 1984; Nakao et al., 1987; Cheriex et al., 1989; Kircher et al., 1990).

From the above, it follows that the change in ultrasonic inferior vena cava indices provides quantitative information on the rapid changes in vascular filling state induced by volume loading. Whether this new method is also suitable for estimating slow changes in vascular filling state in underfill (for example, in shock) or overfill (for example, in heart failure, pregnancy) requires further study. Although in these situations fluid shifts between intravascular and extravascular fluid may occur, the inferior vena cava dimensions may still provide an estimate of the intravascular volume. This is more so since blood volume remains evenly distributed over the venous compartment during slow changes irrespective of filling state (Boekkooi et al., 1991). This method therefore seems promising for use in clinical critical care.

In summary, the ultrasonic inferior vena cava indices are unsuitable for estimating baseline blood volume in healthy subjects. However, imposed acute changes in vascular filling state in these subjects, can be followed reliably by serial measurement of the changes in inferior vena cava diameter $r_{\text {maximal }}$.

\subsection{Acknowledgements}

This study was supported by Schering Nederland BV. We thank Dr. P.A. Stewart for providing a critical review of the manuscript. 
Chapter 5

\section{VOLUME-DEPENDENT ECHOCARDIO-}

GRAPHIC PARAMETERS ARE UNSUITABLE

FOR ESTIMATING BASELINE BLOOD

VOLUME BUT ARE USEFUL FOR DETECTING

ACUTE CHANGES IN VASCULAR FILLING

\section{STATE}

Johannes J Duvekot $\mathrm{MD}^{1}$, Emile C Cheriex $\mathrm{MD}^{2}$, Wie Djin Tan $\mathrm{MD}^{3}$,

Guido AK Heidendal MD, $\mathrm{PhD}^{3}$, and Louis LH Peeters MD, $\mathrm{PhD}^{1}$

From the Departments of Obstetrics and Gynecology ${ }^{1}$, Cardiology ${ }^{2}$ and Nuclear ' Medicine $^{3}$, University Hospital Maastricht, PO Box 1918, 6214 BX Maastricht, The Netherlands. 


\subsection{Abstract}

Objective:

Methods:

Results:

Conclusions:
To assess the hypothesis that echocardiographic variables vary as a function of baseline blood volume and change in response to changes in vascular filling state.

We evaluated the relationship between the atrial and ventricular dimensions and left ventricular function, as measured by combined Doppler and M-mode echocardiography on the one hand, and blood volume, as measured by a standard radioactive isotope technique on the other. Furthermore, we determined the effect of an increment in vascular filling state induced by plasma volume expansion. Test subjects were ten male and six female volunteers.

Blood volume/ $\mathrm{kg}$ was the only factor not different between male and female subjects and therefore suitable for our study. None of the echocardiographic variables correlated with blood volume $/ \mathrm{kg}$. Volume loading increased stroke volume, left atrial diameter, right atrial area, left ventricular end-diastolic dimension, mean velocity of circumferential fibre shortening and left ventricular ejection time.

It is concluded that echocardiographic variables are unsuitable for estimating blood volume. Serial measurements of atrial dimensions and left ventricular dimensions are useful for detecting rapid changes in vascular filling state. 


\subsection{Introduction}

Echocardiography and Doppler techniques have created new opportunities to study central hemodynamics non-invasively and with great accuracy (Huntsman et al., 1983). In pregnancy, preload, afterload and contractility change gradually over a period of weeks to months, as has been demonstrated by longitudinal echocardiographic studies (Capeless and Clapp, 1989; Robson et al., 1989a; Clapp et al., 1988; Lowe et al., 1993). The cardiovascular hemodynamic changes in pregnancy have been suggested to be related to the $30-40 \%$ increase in blood volume, a rise which begins in the first trimester of pregnancy and reaches its peak at the beginning of the 3rd trimester (Hytten and Paintin, 1963; Pirani et al., 1973; Thomsen et al., 1993; Whittaker and Lind, 1993). Current noninvasive methods based on weight and length to predict blood volume are hampered by a large error of estimate (Feldschuh and Enson, 1977). Knowledge about the interrelation between changes in echocardiographic data and those in blood volume, taking into consideration vascular filling state, could increase our understanding of conditions of circulatory underfill (e.g. hypovolemic shock) or overfill (e.g. cardiac decompensation, pregnancy). In obstetrics, hemodialysis and traumatology this may provide a better noninvasive method to monitor therapeutic volume correction, since central venous pressure increases only after complete stress relaxation has been implemented. In humans the impact of changes in blood volume on various ultrasonic cardiovascular parameters has usually been qualitatively evaluated (Di Donato et al., 1982; Flessas and Ryan, 1982; Nixon et al., 1982; Curien et al., 1983). Only two studies have reported a correlation between atrial dimensions and blood volume (Cannella et al., 1988; Rector et al., 1990).

The present study was designed to test the hypothesis that echocardiographic parameters vary as a function of baseline blood volume and change in response to imposed rapid changes in vascular filling state. We evaluated the relationship between baseline echocardiographic cardiovascular dimensions, as measured by combined Doppler and M-mode echocardiography on the one hand, and blood volume, as measured by a standard radioactive isotope technique on the other. Furthermore, we determined the effect of an increment in vascular filling state induced by plasma volume expansion.

\subsection{Subjects and methods}

Selection and characteristics of subjects: The study protocol was approved by the medical ethical committee of the University Hospital of Maastricht. After having been given a detailed explanation of the protocol, sixteen subjects agreed to participate in the study and signed an informed consent form. The subjects were healthy, normotensive men and women, age 25 to 33 . The female subjects were all using oral contraceptives. Body weight of the sixteen subjects ranged from $54.5 \mathrm{~kg}$ to $103.5 \mathrm{~kg}$ and mean estimated body surface area from 1.60 to $2.42 \mathrm{~m}^{2}$ (Dubois and Dubois, 1916). All measurements were performed between 3 and 6 p.m. Before the actual 
measurement, the volunteers were investigated and all proved to be hemodynamically normal on the basis of ECGs and echocardiography. In the first six consecutive male subjects, plasma volume was measured using the radioiodinated serum albumin method (Keyes et al., 1973). Since plasma volume measurements before and after volume loading in these subjects indicated no appreciable loss of administered plasma expander (polygeline, Haemaccel ${ }^{\mathrm{R}}$ ) (Davies 1987) from the intravascular space in the first $10 \mathrm{~min}$ after completion of volume loading (mean increase $986 \mathrm{ml}$ ), the plasma volume measurement with ${ }^{125} \mathrm{I}$-albumin was omitted from the protocol for the subsequent 4 male volunteers in this study. The six female volunteers were not submitted to volume loading, because of likely influences of cyclic hormonal changes on blood volume homeostasis and venous tone (Hassan et al., 1990).

The methodology of plasma volume measurement was as follows. One $\mathrm{ml}$ of 5 $\mu \mathrm{Ci}{ }^{125} \mathrm{I}$ human albumin dissolved in saline was administered intravenously (total body dose of irradiation $=0.04 \mathrm{mSv}$ ). After 10,20 and $30 \mathrm{~min}$, blood samples $(5 \mathrm{ml})$ were withdrawn to determine the packed cell volume (vol\%) and the volume specific plasma radioactivity $(\mathrm{mCi} / \mathrm{ml})$. Virtual volume specific plasma radioactivity at the time of bolus injection was calculated, by extrapolation using the sequentially obtained volume specific plasma radioactivity data determined in the three consecutive plasma samples. Plasma volume was calculated by dividing the total injected radioactivity by the virtual volume specific plasma radioactivity at the time of bolus injection. Blood volume was calculated by multiplying plasma volume by (1/1-packed cell volume). After the last sampling of the male volunteers, $1000 \mathrm{ml}$ of plasma expander polygeline was infused intravenously at a rate of $65 \mathrm{ml} \cdot \mathrm{min}^{-1}$, which meant that volume loading was always completed within $15 \mathrm{~min}$. A second set of three consecutive blood samples $(5 \mathrm{ml})$ was collected at ten, 20 and $30 \mathrm{~min}$ after volume loading. Plasma volume after volume loading was calculated by the same method as described above for the baseline plasma volume measurement. It was assumed that red blood cell volume had not changed after volume loading.

Echocardiographic measurements: Combined M-mode, 2-D and Doppler echocardiographic measurements were performed before and directly after volume loading. The echocardiographic recordings were made using a cross-sectional phased array echocardiographic Doppler system (Hewlett-Packard Sonos 500 and 1000). This system utilizes a $2.5 \mathrm{MHz}$ phased array transducer and a $1.9 \mathrm{MHz}$ independent continuous wave transducer. Data were recorded on a recorder strip chart at a paper speed of $25 \mathrm{~mm} / \mathrm{s}$ for M-mode measurements and $50 \mathrm{~mm} / \mathrm{s}$ for Doppler measurements. Standard echocardiographic views were recorded on videotape. All measurements were performed in semi-left lateral position after a few min of rest. To determine cardiac output, flow was measured across the aortic valve. Aortic velocities were recorded using the apical approach. A careful search was made for the maximal velocity. No angle correction was used to standardize measurements. The area under the velocity curve (aortic velocity integral) was traced. The mean aortic velocity integral was calculated from five consecutive beats. The diameter of the aortic valve was measured at the aortic orifice during systole, using M-mode echocardiography in the parasternal long axis plane. M-mode measurements were performed according to the recommendations of the American Society of Echocardiography (Sahn et al., 
1978). Right atrial area and left atrial diameter were measured by 2-D echocardiography. Heart rate was determined from the R-R interval of the electrocardiogram. Mean heart rate was calculated from five consecutive beats.

Blood pressure was recorded before and after volume loading, using an automated cuff sphygmomanometer. Echocardiographic and Doppler measurements were all performed by one experienced cardiologist (author ECC).

Cardiac output was calculated according to the formula:

cardiac output $\left(\right.$ L. min $\left.^{-1}\right)=$ stroke volume $x$ beart rate

Stroke volume was calculated as aortic area $\mathrm{x}$ aortic velocity integral. Aortic area was calculated as $1 / 4 \pi$ (aortic valve diameter) ${ }^{2}$.

The mean velocity of circumferential fibre shortening (mean Vcf) was calculated using the formula (Fortuin et al., 1972):

mean $\operatorname{Vcf}\left(\right.$ diam. $\left.^{-1}\right)=($ LVEDD-LVESD $) /($ LVET $x$ LVEDD $)$

where LVESD represents left ventricular end-systolic dimension, LVEDD left ventricular end-diastolic dimension and LVET left ventricular ejection time. LVET was calculated from the aortic velocity tracings.

Mean arterial pressure was calculated as diastolic blood pressure + (systolic blood pressure - diastolic blood pressure) $/ 3$.

Statistical methods: Baseline data obtained in male and female subjects who underwent plasma volume determinations were compared using the Mann-Whitney $\mathrm{U}$ test. If a given variable did not differ between males and females, the 12 data points of that echocardiographic variable were pooled. Correlations between thevarious echocardiographic dimensions and baseline plasma volume and blood volume indexed for either weight or body surface area were evaluated by Spearman correlation analysis.

Absolute changes in the variables in response to volume loading were evaluated by means of a paired Student $t$ test. Changes in cardiac dimensions in subjects with an appreciable increase in cardiac output $(>5 \%)$ in response to volume loading were compared with those in subjects without a measurable change in cardiac output by means of the Mann-Whitney U test. A probability ( $p$ value) of less than $5 \%$ was considered significant. All data are presented as means \pm SD throughout the text, unless otherwise stated. 


\subsection{Results}

Mean values of body surface area, packed cell volume and all blood volume variables are listed in Table I.

Table I Mean (SD) values for body surface area $\left(m^{2}\right)$, packed cell volume (vol\%), blood volume and plasma volume ( $\mathrm{ml}$ ) in six male and six female subjects. Plasma volume was measured using the radioiodinated serum albumin metbod.

\begin{tabular}{llll}
\hline & All & Male & Female \\
\hline body surface area & $1.91 \pm 0.21$ & $2.07 \pm 0.17$ & $1.74 \pm 0.08^{\circ}$ \\
packed cell volume & $40 \pm 4$ & $42 \pm 3$ & $38 \pm 3^{\circ}$ \\
blood volume & $5391 \pm 980$ & $6159 \pm 760$ & $4623 \pm 348^{\circ}$ \\
blood volume $/ \mathrm{kg}$ & $74.5 \pm 5.0$ & $75.4 \pm 4.8$ & $73.1 \pm 6.2$ \\
blood volume $/ \mathrm{m}^{2}$ & $2810 \pm 242$ & $2968 \pm 182$ & $2653 \pm 190^{\circ}$ \\
plasma volume & $3158 \pm 454$ & $3536 \pm 308$ & $2780 \pm 128^{\circ}$ \\
plasma volume $/ \mathrm{kg}$ & $43.7 \pm 4.1$ & $43.5 \pm 3.7$ & $44.1 \pm 4.0$ \\
plasma volume $/ \mathrm{m}^{2}$ & $1652 \pm 103$ & $1709 \pm 100$ & $1596 \pm 76$ \\
\hline
\end{tabular}

$\cdot p<0.05 v$ male (Mann-Wbitney $U$ test).

Body surface area, blood volume $/ \mathrm{m}^{2}$, blood volume and plasma volume were larger in the male subjects. The difference in plasma volume $/ \mathrm{m}^{2}$ did not reach significance $(\mathrm{p}=0.054)$, indicating that the difference in blood volume $/ \mathrm{m}^{2}$ was a result of the higher packed cell volume and total volume of red cells in the male subjects. Weight-specific blood volume did not differ between the male and female subjects and was therefore the only parameter suitable for the purposes of our study. Table II lists median values with their ranges for all cardiac dimensions. Left atrial diameter $/ \mathrm{kg}$ and left atrial diameter $/ \mathrm{m}^{2}$ were consistently smaller in the male subjects compared to the female subjects. In contrast, none of the right atrial parameters showed a consistent difference. LVEDD was larger in the male subjects. After combination of the identical variables, none of the echocardiographic dimensions correlated with baseline blood volume $/ \mathrm{kg}$ when evaluated by Spearman regression analysis. Unfortunately, data on myocardial contractility (LVET, mean Vcf) were not available for the female subjects.

Table III lists mean values \pm SD for plasma volume, packed cell volume and blood volume for the first six consecutive male volunteers, determined before and after volume loading. Ten min after completion of volume loading the mean increase in measured plasma volume was $986 \mathrm{ml}$ (range: 779 - $1301 \mathrm{ml}$ ), thus resulting in individual increases in blood volume of $13-18 \%$.

The echocardiographic data before and after volume loading are summarized in Table III. Stroke volume had increased significantly, but this increase did not lead to a consistent increase in cardiac output. Right atrial area, left atrial diameter and LVEDD increased in all subjects, but LVESD remained essentially unchanged. Mean Vcf can be considered a good index for myocardial contractility (Devereux and Reichek, 1977; Stewart et al., 1985). This parameter increased significantly together with the LVET. Afterload did not change, as indicated by the minor and inconsistent 
changes in mean arterial pressure.

In four of the ten subjects subjected to volume loading, cardiac output increased by more than $5 \%$ in response to volume loading. In these four subjects stroke volume also increased by more than $5 \%$, whereas changes in stroke volume were negligible in the other subjects. The baseline echocardiographic dimensions for these two subgroups did not differ appreciably on the Mann-Whitney U test.

Table II Medians and ranges in the first 6 male and all female subjects for all ultrasonic cardiovascular indices. Groups were compared by Mann-Wbitney $U$ test.

\begin{tabular}{|c|c|c|c|c|c|}
\hline \multirow[b]{2}{*}{ heart rate (bpm) } & \multicolumn{2}{|l|}{ Male } & \multicolumn{2}{|c|}{ Female } & \multirow{2}{*}{$\begin{array}{l}\text { P value } \\
0.87\end{array}$} \\
\hline & 66 & $(58-84)$ & 72 & $(54-88)$ & \\
\hline stroke volume $(\mathrm{ml} / \mathrm{min})$ & 83.5 & $(71-115)$ & 78.0 & $(72-90)$ & 0.42 \\
\hline stroke volume $/ \mathrm{m}^{2}$ & 41.0 & $(37.9-48.3)$ & 45.4 & $(40.8-50.4)$ & 0.11 \\
\hline cardiac output (L/min) & 5.60 & $(5.22-7.50)$ & 6.13 & $(4.17-6.55)$ & 0.87 \\
\hline cardiac index $\left(\mathrm{L} / \mathrm{min} \cdot \mathrm{m}^{2}\right)$ & 2.75 & $(2.54-3.20)$ & 3.44 & $(2.36-3.74)$ & 0.20 \\
\hline left atrial diameter (mm) & 38 & $(31-40)$ & 36 & $(31-37)$ & 0.19 \\
\hline left atrial diameter $/ \mathrm{kg}$ & 0.45 & $(0.39-0.49)$ & 0.53 & $(0.52-0.66)$ & 0.004 \\
\hline left atrial diameter $/ \mathrm{m}^{2}$ & 17.5 & $(16.6-19.1)$ & 20.1 & $(18.2-22.5)$ & 0.02 \\
\hline right atrial area $\left(\mathrm{cm}^{2}\right)$ & 18.3 & $(14.2-21.3)$ & 15.0 & $(11.1-15.8)$ & 0.09 \\
\hline right atrial area $/ \mathrm{kg}$ & 0.22 & $(0.18-0.27)$ & 0.22 & $(0.19-0.25)$ & 1.00 \\
\hline right atrial area $/ \mathrm{m}^{2}$ & 8.7 & $(7.1-10.4)$ & 8.4 & $(6.5-8.7)$ & 0.29 \\
\hline LVEDD (mm) & 52 & $(51-54)$ & 45.5 & $(39-50)$ & 0.004 \\
\hline LVEDD/kg & 0.65 & $(0.49-0.78)$ & 0.72 & $(0.67-0.81)$ & 0.08 \\
\hline LVEDD $/ \mathrm{m}^{2}$ & 25.5 & $(21.4-28.8)$ & 25.9 & $(24.4-27.5)$ & 0.52 \\
\hline LVESD (mm) & 33 & $(30-35)$ & 30 & $(26-34)$ & 0.06 \\
\hline LVESD/kg & 0.41 & $(0.29-0.51)$ & 0.47 & $(0.43-0.51)$ & 0.05 \\
\hline $\mathrm{LVESD} / \mathrm{m}^{2}$ & 16.0 & $(12.6-18.7)$ & 16.9 & $(16.2-18.7)$ & 0.15 \\
\hline
\end{tabular}


Table III Echocardiograpbic M-mode and Doppler parameters before and after volume loading witb 1000 ml of plasma expander in 10 male volunteers. Plasma volume, packed cell volume, and blood volume before and $10 \mathrm{~min}$ after $1000 \mathrm{ml}$ volume loading in the first six male subjects. Values are means (SD).

\begin{tabular}{|c|c|c|}
\hline & Before volume loading & After volume loading \\
\hline heart rate (bpm) & $65 \pm 9$ & \pm 10 \\
\hline stroke volume $(\mathrm{ml} / \mathrm{min})$ & $91 \pm 15$ & $97 \pm 14^{\circ}$ \\
\hline cardiac output (L/min) & $5.86 \pm 0.96$ & $6.06 \pm 1.30$ \\
\hline aortic valve diameter $(\mathrm{cm})$ & $2.3 \pm 0.1$ & $2.3 \pm 0.1$ \\
\hline aortic velocity integral & $22.3 \pm 2.7$ & $24.0 \pm 2.4^{*}$ \\
\hline left atrial diameter (mm) & $37.7 \pm 3.4$ & $40.9 \pm 3.2^{* *}$ \\
\hline $\operatorname{LVEDD}(\mathrm{mm})$ & $52.5 \pm 2.8$ & $53.0 \pm 2.5^{\circ}$ \\
\hline $\operatorname{LVESD}(\mathrm{mm})$ & $33.3 \pm 2.4$ & $33.1 \pm 2.2$ \\
\hline right atrial area $\left(\mathrm{cm}^{2}\right)$ & $18.4 \pm 2.9$ & $20.3 \pm 2.5^{\circ}$ \\
\hline LVET (ms) & $299 \pm 11$ & $308 \pm 11^{*}$ \\
\hline mean Vcf (diam/s) & $1.1 \pm 0.1$ & $1.2 \pm 0.1^{*}$ \\
\hline mean arterial pressure $(\mathrm{mmHg})$ & $99 \pm 22$ & \pm 12 \\
\hline plasma volume (ml) & $3536 \pm 309$ & $4522 \pm 394 *$ \\
\hline packed cell volume (\%) & $42 \pm 3$ & $39 \pm 2 *$ \\
\hline blood volume (ml) & $6159 \pm 760$ & $7141 \pm 888 *$ \\
\hline
\end{tabular}

$\cdot p<0.05, \cdot p<0.01 v$ baseline (paired $t$ test)

\subsection{Discussion}

The objective of the present study was to determine whether the echocardiographic dimensions and the changes in these indices induced by volume loading could provide information about baseline blood volume and magnitude of changes in vascular filling state, respectively. The first part of the study explored a possible covariation between blood volume and echocardiographic dimensions. Dynamic echocardiographic parameters can be expected to be influenced to a larger extent by factors other than plasma volume and/or blood volume, such as viscosity and peripheral flow demands (Daniel et al., 1986). The static echocardiographic parameters, like atrial and vena caval dimensions, which do not change with the cardiac cycle, seemed to be more appropriate for the purpose of the present study, as they are integrated into the volume-regulation mechanisms (Rothe 1983). As for the atrial dimensions, we have demonstrated in a previous study that the inferior vena cava diameter varied independently of plasma volume and blood volume (Duvekot et al., 1994b). The correlations between blood volume and the atrial dimensions reported in two previous studies are open to challenge, as they were determined without providing evidence, 
for example by Spearman correlation analysis, that their data were normally distributed (Cannella et al., 1988; Rector et al., 1990). Secondly, possible differences between men and women were not taken into account either. Our study showed no clear differences between the sexes for left atrial diameter or right atrial area, but blood volume was found to be larger in the male subjects (table II). Using multiple regression analysis on our data, introducing blood volume as the dependent variable and sex and left atrial diameter as independent variables, sex accounted for $67 \%$ of the variance, whereas left atrial diameter only accounted for $14 \%$ of the variance. Introducing the weight of the subjects as a variable resulted in the disappearance of significance for sex and left atrial diameter, weight explaining $91 \%$ of the variance in blood volume. It follows that atrial dimensions can only be used to estimate blood volume separately for men and women, with weight probably the most important determinant.

In the second part of our study, we evaluated the changes in cardiac dimensions in response to a rapidly imposed rise in vascular filling state. The advantage of rapid volume loading is that measurements can be performed before any interindividual variation in velocity of volume dissipation can introduce additional variation. The increases found in our study were slightly larger than could be expected from the results of former studies on volume loading with plasma expanders (Van de Broek et al., 1989), which was undoubtedly due to the rapid infusion rate. In most reports, volume loading was employed at a slower rate by using plasma expanders, thus lowering packed cell volume and blood viscosity (Fortuin et al., 1972; Flessas and Ryan, 1982; Curien et al., 1983) or intrinsic volume expansion, e.g., by the headdown-tilt method (Nixon et al., 1982) or water immersion method (Kinney et al., 1987). Furthermore, the present study showed neither heart rate nor mean arterial pressure to change consistently in response to volume loading. This supports the theory that these effects of the volume receptor reflexes require a longer period to develop their full extent and indicating that the hemodynamic effects were confined to the venous side of the circulation. The first response of the volume-regulation system to excess filling is the accomodation of the surplus by filling the large central veins (Guyton et al., 1973c; Rothe 1983; Boekkooi et al., 1991). If mean circulatory filling pressure is not raised, cardiac output will not increase in response to volume loading. In fact, as shown in Table III, cardiac output was found to have changed inconsistently, suggesting that the $1000 \mathrm{ml}$ volume load did not exceed the storage capacity of the venous compartment. This phenomenon of venous distension without concomitant rise in intraluminal pressure has been defined as "stress relaxation" of veins (Prather et al., 1969; Moreno et al., 1973). The lack of a consistent effect on cardiac output may be related to its dependence on heart rate as well. It follows that, in the present experiment, only the immediate effects of an increased preload were observed, thus creating a model of a true overfill situation of the vascular bed.

The increase in blood volume by as much as $18 \%$ resulted in much smaller increases in echocardiographic indices. Obviously, volume-sensitive variables reached maximum width after a certain volume of plasma expander had been administered. The pressure/volume curve of the left ventricle and left atrial diameter is known to follow a curvilinear or exponential course (Di Donato et al., 1982; Curien 
et al., 1983). On the basis of this concept it was expected that the subjects with an increased cardiac output after volume loading would have smaller echocardiographic dimensions, especially left atrial diameter, right atrial area and LVEDD. However, the baseline dimensions were found not to differ for these two subgroups. The latter observation, in combination with the absence of correlation between absolute and percentual increase in blood volume and the ultrasonic dimensions, indicates that the baseline cardiac dimensions lack predictive value with respect to the hemodynamic response to a known amount of volume load.

In summary, echocardiographic indices are unsuitable for estimating baseline blood volume in healthy subjects. Imposed (acute) changes in vascular filling state in these subjects can be reliably detected by measuring changes in echocardiographic parameters. Quantitative changes in vascular filling state can only be detected by serial M-mode measurements of the atrial and left ventricular dimensions if changes in blood volume are small. Whether these parameters are also useful in pregnancy, to follow changes in vascular filling state and preload, requires additional validation for that particular condition.

\subsection{Acknowledgements}

This study was financially supported by Schering Nederland BV. 
Chapter 6

\section{EARLY-PREGNANCY CHANGES IN HEMODYNAMICS AND VOLUME HOMEO- STASIS ARE CONSECUTIVE ADJUSTMENTS TRIGGERED BY A PRIMARY FALL IN SYSTE- MIC VASCULAR TONE}

Johannes J Duvekot $\mathrm{MD}^{1}$, Emile C Cheriex $\mathrm{MD}^{2}$, Frans AA Pieters $\mathrm{MD}^{2}$, Paul PCA Menheere $\mathrm{PhD}^{3}$ and Louis LH Peeters MD, $\mathrm{PhD}^{1}$

From the Departments of Obstetrics and Gynecology ${ }^{1}$, Cardiology ${ }^{2}$ and Clinical Chemistry33, University Hospital Maastricht, PO Box 1918, 6214 BX Maastricht, The Netherlands.

Presented in part at the Thirty-ninth Annual Meeting of the Society for Gynecologic Investigation, San Antonio, Texas, March 18-21, 1992.

American Journal of Obstetrics and Gynecology 1993; 169: 1382-1392. 


\subsection{Abstract}

Objective:

\section{Study design:}

Results:

\section{Conclusion:}

The purpose of this study was to test the hypothesis that early pregnancy changes in volume homeostasis develop as a consequence of preceding changes in maternal hemodynamics.

Maternal cardiovascular function of 10 pregnant women was followed up by Doppler echocardiography. Vascular filling state and volume homeostasis were evaluated by echocardiographic index values, 24-hour creatinine clearance, serum osmolality and volume-regulating hormones. Studies were performed weekly in early pregnancy, in the second and third trimesters and post partum. Changes relative to the fifth week and the consistency of changes between weeks 5 and 8 were evaluated by nonparametric statistics.

In early pregnancy cardiac output increased and afterload decreased. Concomitant increases in ultrasonic preload index values and 24-hour creatinine clearance were accompanied by decreases in serum renin, sodium and osmolality.

These data support the concept that maternal hemodynamic adaptation to pregnancy is most likely triggered by a primary fall in systemic vascular tone. The resulting rapid fall in in preload and afterload leads to a compensatory increase in heart rate and activation of the volume-restoring mechanisms. Subsequently cardiac output increases because of a rise in stroke volume, which develops because the vascular filling state normalizes, whereas the reduced afterload reduction is maintained. 


\subsection{Introduction}

In the course of human pregnancy, plasma volume increases gradually to a plateau of $1250 \mathrm{ml}$ above nonpregnant levels in the 3rd trimester (Hytten et al., 1963; Chesley 1972; Pirani et al., 1973; Whittaker and Lind, 1993). That this particular hemodynamic adaptation is important for pregnancy is emphasized by the high incidence of defective plasma volume expansion in pregnancies complicated by preeclampsia or poor fetal growth (Hays et al., 1985b). Nevertheless, the mechanisms regulating plasma volume expansion in pregnancy are still unknown. In the nonpregnant state plasma volume expansion can be provoked, either mechanically by stretch or pressure exerted on the volume-receptors (for example, in congestive heart failure, arteriovenous shunt) or, to a lesser extent, hormonally by altering the function of the volume regulatory system (for example, in hyperaldosteronism). One can expect that either one or both mechanisms are also involved in the plasma volume expansion of pregnancy.

Most of the $30 \%$ to $60 \%$ increase in cardiac output and at least half of the approximately $10 \%$ fall in arterial pressure in human pregnancy is accomplished in the first trimester (Clapp et al., 1988; Capeless and Clapp, 1989). Whether these hemodynamic changes are directly responsible for the early pregnancy adaptations in the volume homeostasis and renal function is unclear. Such a causal relationship would provide support for a mechanical stimulus for plasma volume expansion, as appears to be the case in the pregnant baboon (Phippard et al., 1986). However, the dissimilarity between gradual pattern of plasma volume expansion up to the thirthieth week (Hytten et al., 1963) and the rapid and early pattern of change in cardiac output and mean arterial pressure (MAP, $\mathrm{mmHg}$ ) contradict a mechanical cause for the plasma volume expansion in human gestation (Robson et al., 1989a). To our knowledge, the changes in cardiovascular, renal and endocrine function have never been measured simultaneously during the period of most pronounced changes in these functions, the early first trimester. Such information would improve our insight into the physiologic mechanisms regulating the adaptation of renal function and volume homeostasis in human pregnancy.

The current study was designed to test the hypothesis that pregnancy-related changes in renal function and volume homeostasis, develop early in pregnancy and are secondary to, rather than independent of, the adaptive changes in maternal hemodynamics. To this end, we determined serially in 10 subjects who subsequently were found to have normal pregnancies hemodynamic function, vascular filling state, renal function and related steroid levels.

Hemodynamic function was evaluated by the measurement of a number of static and dynamic cardiac parameters by means of combined M-mode and continuouswave Doppler echocardiography (Huntsman et al., 1983; Robson et al., 1987d). About 10 minutes after these measurements, the MAP was measured with a cuff sphyngomanometer with the patient in sitting position. Changes in the vascular filling state were estimated indirectly by means of the changes in the ultrasonic dimensions of the inferior vena cava, left ventricular end-diastolic dimension (LVEDD) and left atrial diameter in combination with plasma renin concentration and alpha atrial natri- 
uretic protein ( $\alpha-\mathrm{ANP})$. Because hemodilution can be expected to play a role in the gestational changes in volume homeostasis (Davison et al., 1981b; Schrier and Briner, $1991)$, the serum osmolality $(\mathrm{mOsm} / \mathrm{l})$, sodium $(\mathrm{mmol} / \mathrm{l})$, potassium $(\mathrm{mmol} / \mathrm{l})$, urea $(\mathrm{mmol} / \mathrm{l})$, protein $(\mathrm{g} / \mathrm{l})$ and albumin $(\mathrm{g} / \mathrm{l})$ were determined as well. Renal function was evaluated by measuring the 24-hour creatinine clearance (glomerular filtration rate, $\mathrm{ml} / \mathrm{min})$, serum creatinine $(\mu \mathrm{mol} / \mathrm{l})$, and fractional salt excretion (\%). Finally, changes in the early pregnancy steroid environment were determined on the basis of levels of serum 17ß-estradiol, 17-hydroxyprogesterone, and progesterone.

\subsection{Subjects and Methods}

Selection and characteristics of subjects. Healthy, normotensive subjects $\leq 40$ years old attending our subfertility clinic were invited in consecutive order to participate in this study as soon as pregnancy was established. Pregnancy was confirmed by a sensitive urinary human chorionic gonadotropin determination. A total of 22 women signed an inform consent form and were subsequently enrolled in this study. Conceptional dates were either based on ultrasonic ovulation detection or were calculated on the basis of an early crown-rump length measurement. The amenorrhea (in weeks) was standardized by adding 2 weeks to the conceptional date.

Eight subjects aborted spontaneously before the tenth week of pregnancy and four had severe IUGR in late pregnancy. Data from these 12 women were omitted. The remaining 10 (singleton) pregnancies were normal and resulted in the birth of healthy newborns ranging in weight between the 10th and 90th percentiles for our obstetrical population (Kloosterman 1970). General characteristics of these subjects are listed in Table I.

Table I Characteristics of ten subjects with normal pregnancies. Values are expressed as means with the range given in parentbeses.

\begin{tabular}{lll}
\hline maternal age $(\mathrm{yr})$ & 29.1 & $(23-34)$ \\
maternal height $(\mathrm{cm})$ & 167 & $(154-175)$ \\
maternal prepregnancy weight $(\mathrm{kg})$ & 65.9 & $(52.5-82)$ \\
gestational age at delivery (weeks) & 40.0 & $(38.3-40.6)$ \\
newborn weight $(\mathrm{g})$ & 3411 & $(2870-3990)$ \\
nulliparity & 7 & \\
ovulation induction & 2 &
\end{tabular}

Two subjects conceived after ovulation induction by clomiphene citrate. Seven subjects were nulliparous and 3 were multiparous. Initial hemodynamic function was normal in all subjects, as indicated by normal electrocardiogram and echocardiography at first cardiovascular study at 5 weeks' amenorrhea ( $35 \pm 3$ days). Studies were performed weekly between the fifth and tenth weeks; at 14, 25 and 35 weeks of pregnancy; and either three months post partum or after discontinuation of breastfeeding. 
Echocardiographic methods. All echocardiographic measurements were performed in semi-left lateral position after approximately 5 minutes of rest. The echocardiographic recordings were made with a cross-sectional, phased array echocardiographic Doppler system (Hewlett-Packard Sonos 500 and 1000). This system utilizes a $2.5 \mathrm{MHz}$ phased array transducer with continuous-wave and a $1.9 \mathrm{MHz}$ independent continuous-wave transducer. Data were registered on a strip chart recorder at a paper speed of $25 \mathrm{~mm} . \mathrm{s}^{-1}$ for M-mode and at $50 \mathrm{~mm} . \mathrm{s}^{-1}$ for Doppler measurements. Standard echocardiographic views were recorded on videotape. M-mode measurements were performed according to the recommendations of the American Society of Echocardiography (Sahn et al., 1978).

Measurements and calculations. Cardiac output was calculated according to the formula

\section{Cardiac output $\left(\right.$ L. $\left.\mathrm{min}^{-1}\right)=$ stroke volume $x$ beart rate}

To determine stroke volume, flow was measured across the aortic valve. Aortic velocities were recorded by means of the apical approach. A careful search for the maximal velocity was made. No angle correction was used to standardize measurements. The area under the velocity curve (aortic velocity integral) was recorded on disc. The mean aortic velocity integral was calculated with five consecutive ejections. The diameter of the aortic valve was measured off-line at the aortic orifice during systole with the M-mode echocardiographic aortic valve separation. Stroke volume was calculated as aortic area $\mathrm{x}$ aortic velocity integral. The aortic area was calculated as $1 / 4 \pi$ (aortic diameter) $)^{2}$. In pregnancy aortic diameter ranged from $1.92 \pm 0.12$ to 1.98 \pm 0.16 with inconsistent trend. Therefore it was decided to use the mean of the 10 aortic diameter values measured in each subject to compute stroke volume. Heart rate was calculated by averaging five consecutive R-R intervals on the ECG. Left ventricular mass was calculated according to the D-cubed formula of Devereux and Reichek (Devereux and Reichek, 1977):

\section{left ventricular mass $(\mathrm{gm})=1.04(\text { LVEDD }+P W T+I V S T)^{3}-(\text { LVEDD })^{3}-13.6$}

where LVEDD represents left ventricular end-diastolic dimension, PWT is posterior left ventricular wall thickness, and IVST is the interventricular septal thickness.

The mean velocity of circumferential fibre shortening (mean Vcf) provides an indirect estimate of myocardial contractility and was calculated with the formula (Fortuin et al., 1972):

mean $\operatorname{Vcf}\left(\right.$ diam..$\left.^{-1}\right)=($ LVEDD-LVESD $) /($ LVETxLVEDD $)$

where LVESD represents left ventricular end-systolic dimension and LVET the left ventricular ejection time, which was calculated from the aortic velocity tracings.

Total peripheral vascular restistance (TPVR) was calculated as follows:

$\operatorname{TPVR}\left(\right.$ dyn $\left.\cdot \mathrm{s}^{-1} \cdot \mathrm{cm}^{-5}\right)=\operatorname{MAP}(\mathrm{mmHg}) \times 80 /$ cardiac output $\left(\mathrm{L} \cdot \mathrm{min}^{1}\right)$ 
In this formula MAP was calculated as the diastolic blood pressure plus one third of the pulse pressure.

After the echocardiographic measurements, the ultrasonic dimensions of the inferior vena cava were determined from the subxyphoidal region with the subject in the supine position. Long and short axis views of the inferior vena cava were obtained just below the diaphragm in the hepatic segment (Cheriex et al., 1989). The maximal inferior vena cava diameter ( $\left.I V C D_{\text {maximal }}\right)$ was measured during unforced expiration and before the P-wave on the ECG to avoid interference with the A wave of the venous pressure curve. Maximal emptying of the abdominal inferior vena cava producing the smallest inferior vena cava contour $\left(\mathrm{IVCD}_{\text {small }}\right)$ was obtained during maximal inspiration, when venous drainage towards the thoracal inferior vena cava is maximized in response to the high intrathoracic negative pressure (Cheriex et al., 1989). Most of the blood that occupies the collapsible part of the inferior vena cava will be carried toward the thorax and will be incorporated in the venous return. The collapsible part of the inferior vena cava was defined as IVCD collapsible $_{\text {and defined as }}$ the difference between IVCD $D_{\text {minimal }}$ and IVCD $D_{\text {maximal }}$. As IVCD always the outermost layer of the supposedly more or less round inferior vena cava,

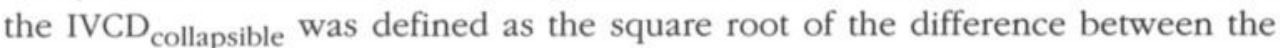
squared IVCD maximal and squared IVCD small $_{\text {. Finally, the IVCD }}$ minimal was defined as

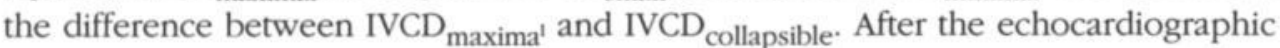
study, which lasted at most 15 minutes, blood pressure was measured in sitting position using a cuff sphygmomanometer. Diastolic blood pressure was taken at the fourth Korotkoff sound. All echocardiographic studies with subsequent blood pressure measurements were performed at random by two experienced cardiologists (coauthors ECC and FAAP) unaware of the subjects' gestational age.

Laboratory measurements. Before ultrasonography blood samples were withdrawn from the antecubital vein in sitting position after approximately 5 minutes of rest. All samples were collected early in the morning. Blood for measurements of electrolytes was withdrawn in $10 \mathrm{ml}$ vacutainer glass tubes (Monoject; Sherwood Medical, St. Louis, Mo.) and that for $\alpha$-atrial natriuretic peptide and plasma renin concentration measurement in $5 \mathrm{ml}$ ethylenediaminetetraacetic acid Vacutainer bloodcollecting tubes and $10 \mathrm{ml}$ Vacutainer blood-collecting tubes, respectively. The latter samples were immediately put on ice and centrifuged at $4^{\circ} \mathrm{C}$ for 10 minutes at $1500 \mathrm{~g}$ within 30 minutes after sampling. Urine samples were always collected during the subsequent 24 hours. All samples were stored at $-20^{\circ} \mathrm{C}$ before assay. Serum levels of sodium, $\mathrm{K}^{*}$, osmolality, creatinine, urea, protein and albumin were measured by standard chemical procedures with a Dimension Clinical Chemistry Analyzer (Du Pont, Wilmirgton, Del.). $\alpha$-Atrial natriuretic peptide was analyzed by radioimmunoassay (ANP RIA; Incstar, Stillwater, Okla.) with a sheep antihuman $\alpha$-ANP antibody for the antigen-antibody reaction and a horse antisheep precipitating complex for the separation step. The first antibody is specific to the ring structure of the molecule and presents crossreactivities of $100 \%$ to $\alpha$-ANP (1-28), $91 \%$ to ANP (4-28), $122 \%$ to ANP (5-28), $81 \%$ to ANP (5-27) and $148 \%$ to ANP (7-28). There is no significant crossreactivity versus other possible interfering substances. The interassay and intraassay reproducibilities are better than $91.2 \%$ and $85.6 \%$ respectively. Plasma renin concentration 
was estimated by the Renin IRMA Pasteur (Marnes la Coquette, France) immunoradiometric assay technique. The renin is allowed to react with a first monoclonal antibody against both active and inactive forms of renin coupled to a solid phase. After incubation, the mixture is washed and then, a second ${ }^{131} \mathrm{~J}$-labelled monoclonal antibody, specific only for active forms of renin is added. After reincubation and rewashing the sandwich complexes are counted. The assay is standardized versus WHO 68/356. The interassay and intraassay reproducibilities are better than $91.4 \%$ and $90.6 \%$, respectively.

Serum 17ß-estradiol, 17-hydroxyprogesterone, and progesterone were determined using reagent kits of DPC (DPC, Los Angeles, Cal.). These assays are based on solidphase radioimmunoassays using coated tube technology. Sensitivity of the assays are $0.035,0.21$ and $0.15 \mathrm{nmol} / \mathrm{l}$, respectively. The assays are not influenced by hemolysis or lipolysis. The results do not suffer from prolonged sample storage periods at - 20 ${ }^{\circ} \mathrm{C}$. For all three substances precision is better than $5 \%$. The progesterone assay does not crossreact with other steroids. The assay for $17 \AA$-estradiol shows crossreactivity with estrone by $10 \%$. The assay for 17-hydroxyprogesterone crossreacts with 11deoxycortisol and progesterone by $1.5 \%$ and $3.5 \%$, respectively. For the purpose of our study we assumed these crossreactivities not important.

Statistical methods. All variables were determined up to nine times in pregnancy and once post partum. Although none of the variables appeared to be skewed by inspection, the limited sample size obstructed statistical confirmation of normal data distribution. Therefore it was considered more appropriate to analyze the data by nonparametric tests, because those tests do not require such a precondition. Data in the text are presented as medians with ranges (between parentheses) unless otherwise stated. Differences relative to the 5 -week value were evaluated with the Wilcoxon signed-rank test. Because the largest change in variables was noted to occur between the fifth and eighth weeks, this period was evaluated more thoroughly. For each of the 10 pregnancies (linear) regression slopes were computed for the relationship between each of the measured variables (dependent) and the gestational age between 5 and 8 weeks (independent). The calculation yielded 10 slopes for each of the variables evaluated. Consistency of the slopes was evaluated by random regression analysis (Laird and Ware, 1982). The "early pregnancy mean" for each variable was defined as the mean of the four data points between 5 and 8 weeks. The difference between this value and those values obtained later in pregnancy and post partum was also evaluated by Wilcoxon signed-rank test. The contribution in each early pregnancy week, of the changes in stroke volume ( $\delta$-stroke volume) and heart rate $(\delta$-heart rate) to that in cardiac output ( $\delta$-cardiac output) was determined by multiple linear regression. Finally, the correlations between the $\delta$-stroke volume, $\delta$-heart rate and $\delta$-cardiac output on the one hand, and the concomitant, weekly changes in potentially related hemodynamic variables in the period between the fifth and tenth weeks on the other hand, were evaluated by stepwise multiple linear regression. Independent variables chosen to be included in this analysis were left atrial diameter, left ventricular mass, mean Vcf and MAP. In the comparisons a probability $<5 \%$ was considered significant. 


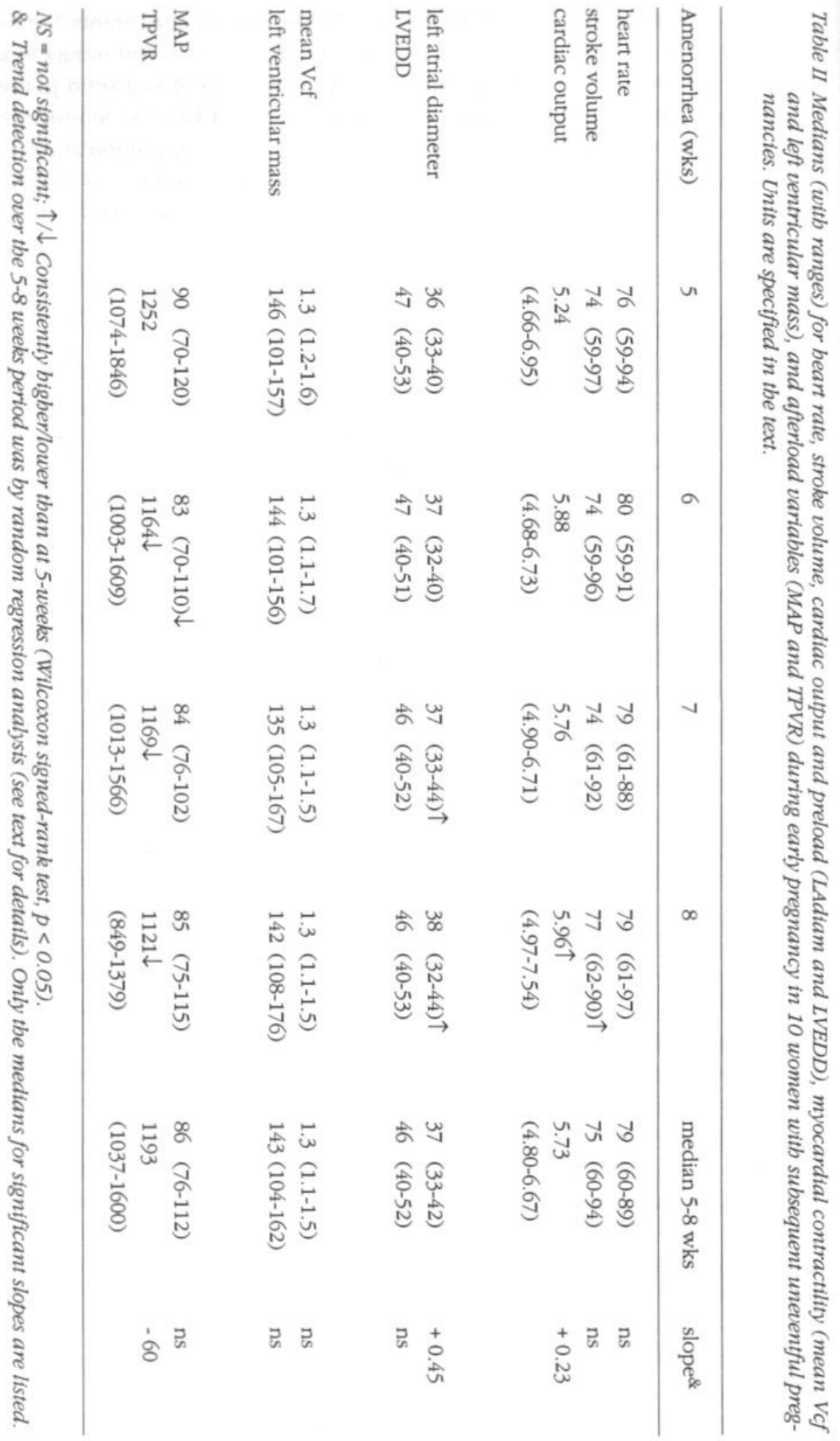




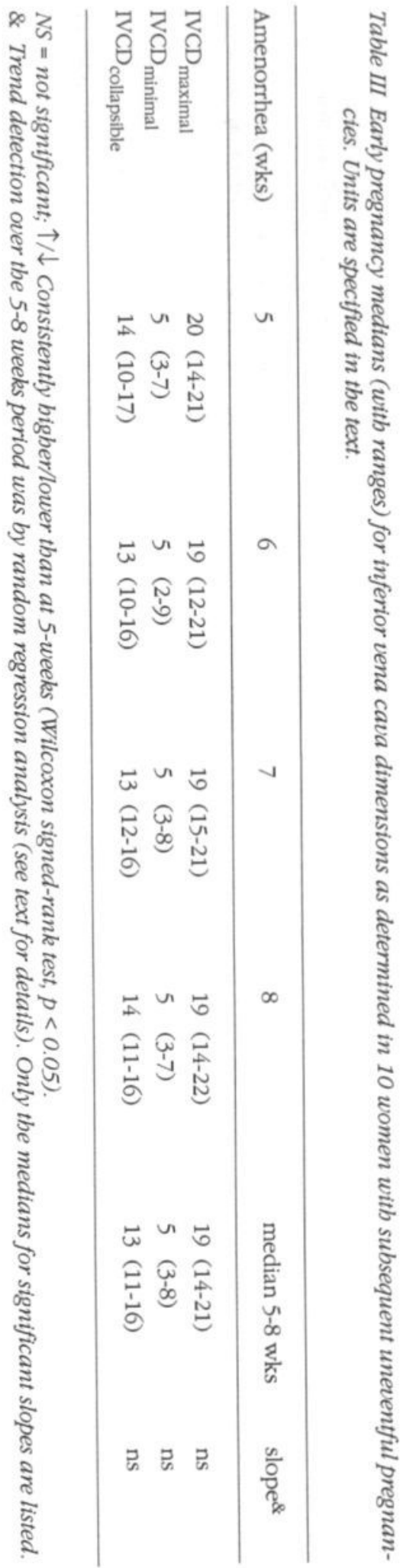




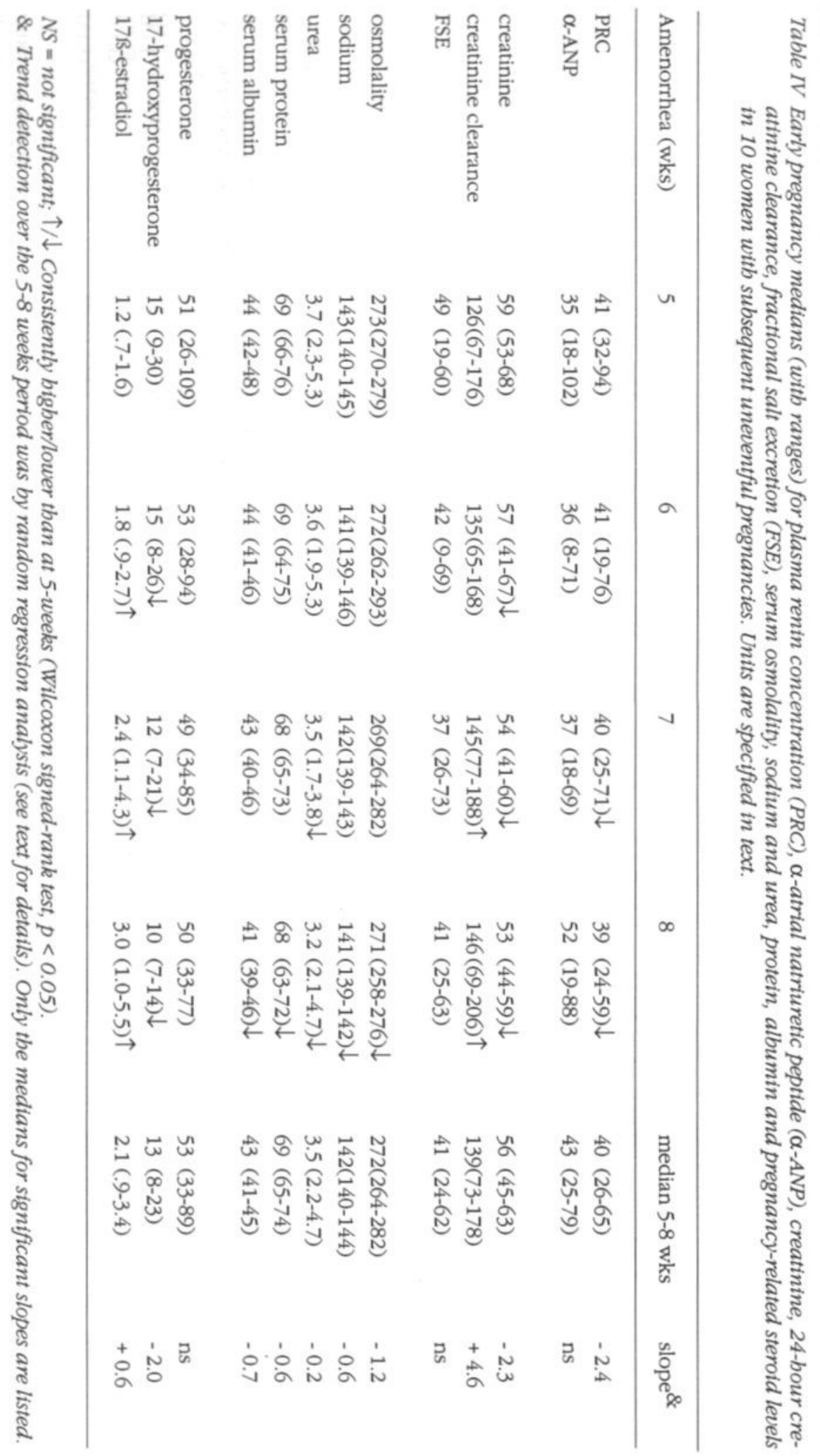




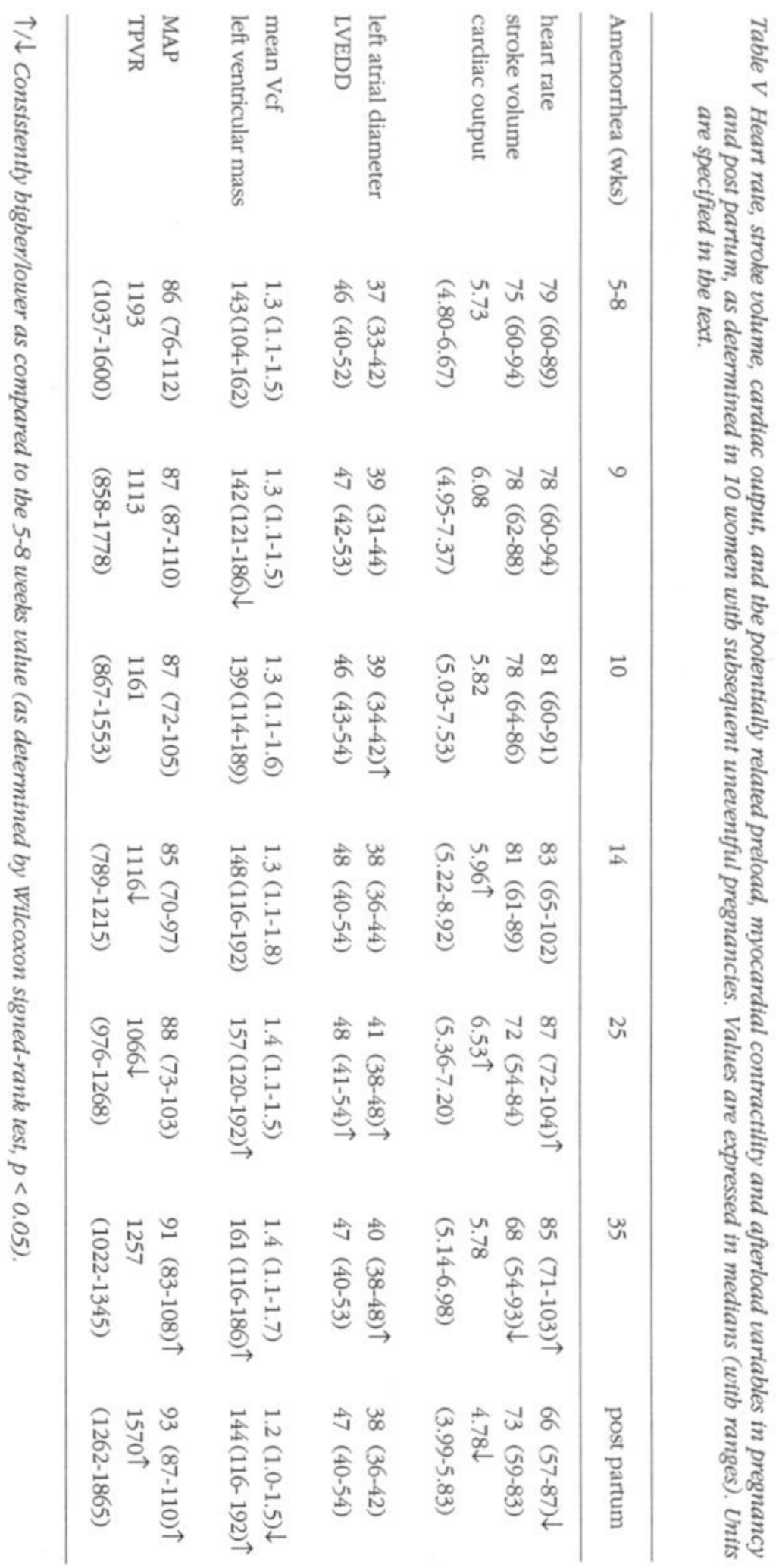




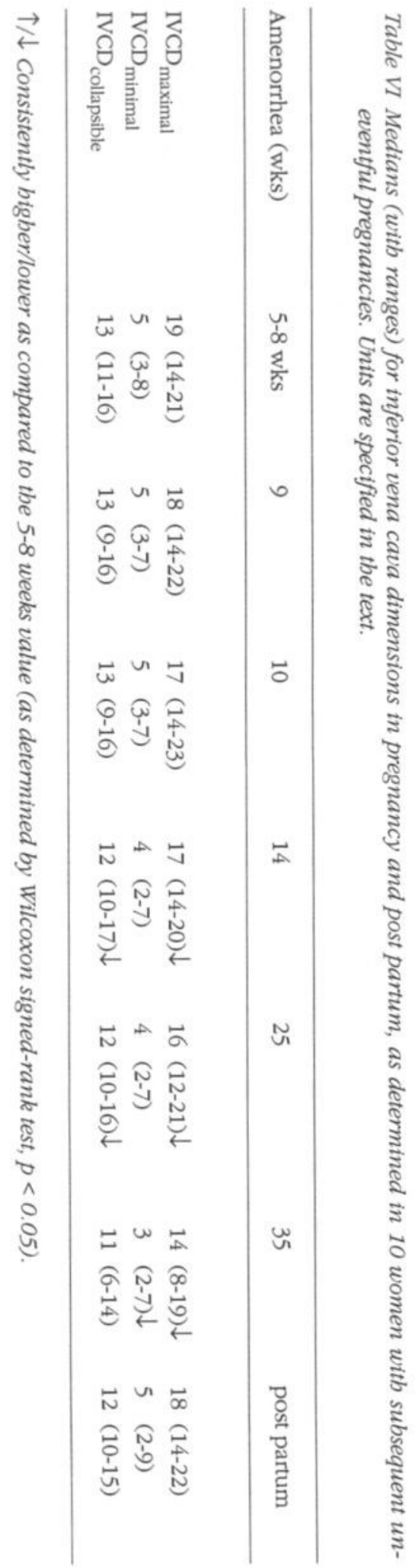




\subsection{Results}

Median values for heart rate, stroke volume and cardiac output in early pregnancy are listed in Table II. By the eighth week both stroke volume and cardiac output had increased relative to the 5-week value. Of heart rate, stroke volume and cardiac output, only cardiac output increased consistently between the fifth and eighth week. In the sixth week (week 5 to 6 ) the variation in stroke volume explained $10 \%$ ( $\delta$-heart rate $90 \%$ ) of the variation in cardiac output. In the seventh, eighth, ninth and tenth weeks, this figure was $36 \%, 53 \%, 30 \%$ and $17 \%$, respectively. The hemodynamic determinants of cardiac output, also listed in Table II, were characterized by the following variables: preload: left atrial diameter and left ventricular end-diastolic dimension, myocardial contractility: mean Vcf and left ventricular mass, and afterload: MAP and total peripheral vascular resistance. Of these variables only left atrial diameter was found to increase -, and total peripheral vascular resistance to decrease consistently, between the fifth and eighth weeks.

Early pregnancy medians with ranges for inferior vena cava dimensions are listed in Table III, whereas those related to the volume homeostasis and renal function are listed, together with concomitant steroid levels, in Table IV. Inferior vena cava dimensions changed little between the fifth and eighth weeks. On the other hand, 24hour creatinine clearance increased and plasma renin concentration decreased consistently, the latter being accompanied by decreases in serum levels of sodium, urea, osmolality, creatinin, protein and albumin. The concomitant trend in $\alpha$-atrial natriuretic peptide and fractional salt excretion with pregnancy was inconsistent. Progesterone changed little, 17-hydroxyprogesterone decreased and 17ß-estradiol increased in early pregnancy.

The medians with ranges of the hemodynamic variables measured after the eighth week and post partum are listed in Table V. Although heart rate increased steadily in the remainder of pregnancy, stroke volume plateaued in midpregnancy and tended to decrease afterward. As a consequence, the rise in cardiac output leveled off by the twenty-fifth week. Of the other hemodynamic variables, left atrial diameter continued to increase after the first trimester. Left ventricular end-diastolic dimension and left ventricular mass, which changed little until the 10th week, tended to increase in the second half of pregnancy. MAP returned almost to post partum values shortly before term. Total peripheral vascular resistance continued to decrease to a nadir at the twenty-fifth week. Table VI illustrates the pattern of change in inferior vena cava dimensions after the eighth week. The IVCD $\mathrm{D}_{\text {maximal }}$ decreased throughout the remainder of pregnancy, in midpregnancy because of an isolated fall in IVCD collapsible, which was only at 35 weeks, accompagnied by a reduction in IVCD $_{\text {minimal. }}$

The relationships between the weekly changes in cardiac output, heart rate, and stroke volume as dependent variables, on the one hand, and the concomitant changes in a number of other obviously independent hemodynamic variables, on the other hand, were analyzed by multiple linear regression. The regression equations calculated by this analysis are listed in Table VII. In the seventh week $\delta$-stroke volume correlated inversely with $\delta$-left atrial diameter. Neither $\delta$-heart rate nor $\delta$-cardiac 
output correlated with any of the evaluated independent variables. The negative correlation between $\delta$-stroke volume and $\delta$-left atrial diameter became positive in the eighth week and was paralleled by a positive correlation between $\delta$-left atrial diameter and $\delta$-cardiac output. The latter positive correlation persisted in the subsequent ninth and tenth weeks. Throughout early pregnancy $\delta$-heart rate varied independently of all evaluated independent variables. Finally, in the tenth week, $\delta$-stroke volume correlated positively with $\delta$-Vcf and negatively with $\delta$-MAP.

Table VII Results of multiple linear regression analysis of the relationship between the weekly changes during early pregnancy in cardiac output, stroke volume, and beart rate as dependent variables, and the potentially related and concomitantly determined bemodynamic variables left atrial diameter (LAdiam), left ventricular mass, mean Vcf and MAP. Only regression coefficients that bave p-values $<0.05$ are quoted. Standard errors for the slope are given in parentheses.

\begin{tabular}{|c|c|c|c|}
\hline $\begin{array}{l}\text { pregnancy } \\
\text { week }\end{array}$ & $\delta$-cardiac output & $\delta$-stroke volume & $\delta$-heart rate \\
\hline $5-6$ & - & - & - \\
\hline $6-7$ & - & $1.6 \delta$-LAdiam $(0.4)$ & - \\
\hline $7-8$ & $+0.2 \delta$-LAdiam $(0.1)$ & $+1.6 \delta$-LAdiam $(0.5)$ & - \\
\hline $8-9$ & $+0.16 \delta$-LAdiam $(0.04)$ & $+1.1 \delta$-LAdiam $(0.4)$ & - \\
\hline $9-10$ & $+0.16 \delta$-LAdiam $(0.06)$ & $+23 \delta$-Vcf $(8)-0.5 \delta$-MAP $(0.2)$ & - \\
\hline
\end{tabular}

The medians with ranges observed after the eighth week and post partum, of the variables related to volume homeostasis and renal function, and the concomitant steroid levels, are listed in Table VIII. Neither the plasma renin concentration nor the 24hour creatinine clearance seemed to continue their change observed in early pregnancy. On the other hand, the serum levels of most variables related to volume homeostasis had persistently fallen to below the level in early pregnancy. The early pregnancy decrease in 17-hydroxyprogesterone seemed to bottom out at 14 weeks, followed by an increase afterward. After the eighth week, both 17ß-estradiol and progesterone increased progressively until late pregnancy.

Comparison of the data points obtained post partum with the early pregnancy mean showed recovery of all pregnancy-induced changes, except for stroke volume, 24-hour creatinine clearance, and serum urea (tables V and VIII). More specifically, cardiac output, heart rate, mean Vcf, plasma renin concentration and the steroid levels had decreased to a level significantly below the early pregnancy reference value whereas the left ventricular mass, MAP, total peripheral vascular resistance, $\alpha$ atrial natriuretic peptide, fractional salt excretion and all other serum levels, had increased to a level significantly above the early pregnancy reference. 


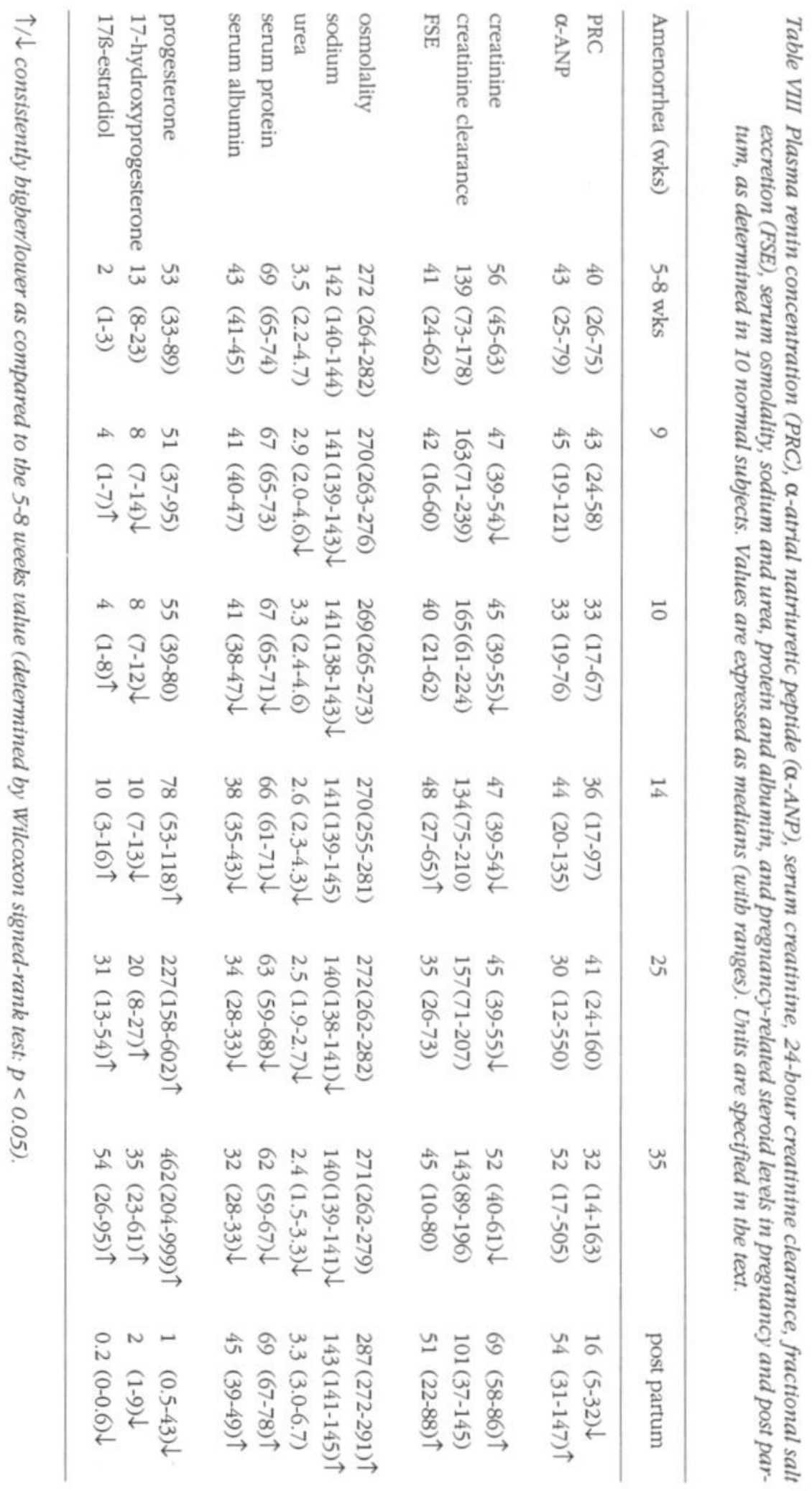




\subsection{Comment}

The objective of this study was to determine whether the early pregnancy changes in renal function and the related changes in volume homeostasis developed independently or as a direct consequence of the early hemodynamic adaptations to pregnancy. None of the measurement procedures used in this study were invasive. The advantage of this approach is minimal risk for the mother and her conceptus and the possibility of performing repeated measurements in this particular period of pregnancy. Conversely, noninvasive procedures are often indirect, which limits their strength, whereas some of the procedures also used in this study, such as the creatinine clearance, blood pressure measurement, and the ultrasonic measurement of small vascular dimensions, are hampered by relatively large measurement errors. Nevertheless, by repeated measurement of a wide spectrum of physiologically related variables the impact of these disadvantages was at least in part neutralized.

Maternal cardiovascular adaptation in early pregnancy was explored on the basis of repeated measurements between the fifth and tenth weeks of pregnancy. We used M-mode combined with continuous-wave Doppler echocardiography. This technique has been well validated to measure central cardiac performance in both nonpregnant (Huntsman et al., 1983; Bouchard et al., 1987) and (predominantly late) pregnant women (Easterling et al., 1987). The use of a suitable echocardiographic measurement technique at the anatomically most appropriate measurement site for the valve cross sectional area is crucial to obtain reliable information for the calculation of cardiac output. In nonpregnant individuals A-mode, M-mode and two-dimensional echocardiography is used to measure aortic cross-sectional area in estimating left ventricular stroke volume. It has been suggested that the accuracy of M-mode echocardiography is limited because of the difficulty in measuring the aortic valve diameter perpendicular to the orifice (Robson et al., 1987d). To standardize measurements, the aortic leaflet separation was measured with M-mode. In normal conditions the separation of the leaflets reflects the aortic annulus diameter accurately. In experienced hands this method has been demonstrated to be more accurate than twodimensional echocardiography (Ihlen et al., 1984; Bouchard et al., 1987). Another important source of error in measuring the aortic cross-sectional area is the site of measurement. Although technically more difficult, it is feasible by M-mode echocardiography to measure the aortic valve orifice at the aortic valve, which is the most suitable site for analysis of cross-sectional area (Ihlen et al., 1984; Bouchard et al., 1987). The aortic valve is composed of fibromuscular tissue and this may explain why cross-sectional area measured at this site and throughout the cardiac cycle, does not change noticeably during pregnancy (Stewart et al., 1985; Bouchard et al., 1987). The latter is supported by our finding that neither the aortic valve size nor the left ventricular systolic and diastolic dimensions had changed in the course of pregnancy. Data on the change in aortic outflow tract diameter in the course of pregnancy are conflicting. In some studies the aortic outflow tract diameter measured by twodimensional or M-mode echocardiography vas found to increase by approximately $1.5 \mathrm{~mm}$ in pregnancy, allowing for an approximately $14 \%$ rise in stroke volume (Robson et al., 1989a). In other studies this increase could not be substantiated 
(Caton and Banner, 1987; Mabie et al., 1994). The conflicting results are hard to explain and may, at least in part, be related to the measurement technique. Some authors neglect this problem by using the aortic velocity integral or stroke distance as the linear analogue of stroke volume (McLennan et al., 1987).

Because prepregnant reference values were not available in the current study, the 5-week value served as a reference for both the other early pregnancy data points and the one obtained post partum. The early pregnancy mean served as a reference for the data points obtained after the eighth week. The differences in most postpartum observations relative to 5 -week values can be interpreted either as related to a persisting change secondary to pregnancy or as indicative of already altered maternal hemodynamics and volume homeostasis in the fifth week of pregnancy. For this reason only those postpartum values were used as nonpregnant reference for which previous reports had provided convincing evidence for complete recovery in the first 3 months post partum. For instance, the observed larger left ventricular mass 3 months post partum is likely to reflect residual left ventricular hypertrophy. This phenomenon, possibly accompanied by a slightly elevated stroke volume may persist for about 6 months post partum (Robson et al., 1987b; Capeless and Clapp, 1991; Robson and Dunlop, 1992). It follows that for the left ventricular mass and stroke volume the observation in the fifth week represents a better reference for evaluating early pregnancy changes than that obtained post partum. In contrast, postpartum heart rate has been reported to return rapidly to the prepregnant level (Robson et al., 1987b; Capeless and Clapp, 1991) which implies that the 3-month postpartum heart rate can be expected to provide a reliable nonpregnant reference. Relative to this reference, the heart rate had already increased by approximately $15 \%$ (from 66 to 76 beats $/ \mathrm{min}$ ) in the fifth week, changed little between 5 and 8 weeks, and resumed its increase after the eighth week, to a maximum of approximately 85 beats $/ \mathrm{min}$ in the thirty-fifth week, a total rise which was comparable with data reported by others (Katz et al., 1978; McLennan et al., 1987; Clapp et al., 1988; Robson et al., 1989a). The apparent biphasic rise in heart rate in the first trimester was also noticable, although not emphasized, in a recent study (Robson et al., 1989a).

After an initial period of little change stroke volume increased consistently between the fifth and eighth weeks, accompanied by a rise in the fractional contribution of stroke volume to the concomitantly increasing cardiac output. After the eighth week the rise in stroke volume leveled off to an apparent plateau in midpregnancy, a pattern that agrees with previous observations (McLennan et al., 1987; Capeless and Clapp. 1989; Robson et al., 1989a). The sequential rise in heart rate before, and in stroke volume after, the fifth week is probably related to a concomitant change in the vascular filling state, the consequences of which will be discussed in detail. At 8 weeks the cardiac output had already increased by $13 \%$ above the 5 -week reference. The initial steep increase weakened, merging by the twenty-fifth week to a plateau of $25 \%$ above the 5-week value. This pattern of change was comparable to that reported by others (Walters et al., 1966; Rubler et al., 1977; Katz et al., 1978; Robson et al., 1989a; Easterling et al., 1990c). 
The interdependence between the adaptation in vascular filling state and that in circulatory function in early pregnancy was explored by assessing in consecutive weeks the covariation between heart rate, stroke volume, and cardiac output changes, on the one hand, and those in various other cardiovascular variables, on the other. The increase in stroke volume that seems to neutralize an initial rise in heart rate (see above), together with a marked rise in the fractional contribution of the stroke volume rise to the concomitant rise in cardiac output between week 5 and 8 of pregnancy, is suggestive of a rise in the vascular filling state in this period. Additional support for this phenomenon is provided by the reversal in the eighth week of the correlation between the variation in stroke volume and that in left atrial diameter, because the left atrial diameter can be expected to vary as a function of preload (Rector et al., 1990), by the consistent increase in left atrial diameter in this period that has also been noted by others (Katz et al., 1978; Robson et al., 1989a; Barth 1990) and by the concomitant fall in plasma renin concentration. Whether the filling state of the vascular bed and cardiac output increase together in response to a yet unknown common factor or, alternatively, whether these two adaptive changes are causally related cannot be determined from the available data. Theoretically an increase in stroke volume can be induced by a rise in venous return, a rise in cardiac contractility, and a fall in afterload (Guyton et al., 1959). It is unlikely that the rise in stroke volume between weeks 5 and 8 in this study was a result of a rise in venous return, first because of the prevailing state of relative hypovolemia and second because of the lack of a concomitant rise in left ventricular end-diastolic dimension, also reported by others (Katz et al., 1978; Barth 1990), which is known to vary as a function of venous return (Curien et al., 1983). Because the cardiac contractility had also not increased appreciably in this period of pregnancy, the afterload reduction should be considered the most likely trigger for the early pregnancy rise in stroke volume. Studies on the adaptation of the vascular filling state in early human pregnancy are lacking, but in the baboon and guinea pig it has been demonstrated that the gestational rise in cardiac output precedes that in blood volume (Hart et al., 1985; Phippard et al., 1986), suggesting that volume retention is probably activated by primary changes in the hemodynamic function.

Data collection in the present study started in the fifth week. Subsequent trends, as specified in the preceding paragraph, indicate that already at that time the vascular bed was underfilled and had already activated volume-conserving mechanisms. The underfilled state of the vascular bed at 5 weeks is supported by the $5 \%$ to $15 \%$ elevation in heart rate at that time (Robson et al., 1989a), which may have been triggered in an attempt to maintain cardiac output in a state of relative hypovolemia. Also the hemodilution already detected in the fifth week in this study and others may have been triggered by an underfilled state of the vascular bed (Davison et al., 1981b; Schrier and Briner, 1991). One can only speculate about the sequence of events that led to the apparent very early hypovolemia. The gradual increase in left atrial diameter without a measurable change in the inferior vena cava diameter suggests an initial fall in vascular tone with a selective increase in the atrial, but not venous, volume at a given filling pressure, because the atrial wall contains more smooth muscle per unit weight than does the venous wall. It is possible that an overall decrease in vascular 
tone in response to a yet unknown endocrine stimulus represents the very first adaptive change of the cardiovascular system, giving rise to both an increased vascular capacity and decreased filling state. The induced compensatory volume retention proceeds slowly, as evidenced by reduced $\alpha$-atrial natriuretic peptide levels in early pregnancy.

Although the inferior vena cava dimensions have been studied extensively in relation to right atrial and ventricular function, no data are available about how they change in pregnancy (Cheriex et al., 1989). In this study the inferior vena cava dimensions had changed little in early pregnancy but decreased afterward, particular-

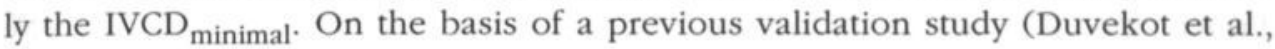
1994b), these changes are consistent with a lower filling state of the vascular bed in late pregnancy. However, it should be stressed that, with advancing pregnancy, compression of the inferior vena cava by the growing uterus may progressively reduce the reliability of these ultrasonic indices (Kerr et al., 1964).

In this study the 24-hour creatinine clearance was found to have increased in the first 8 weeks of pregnancy, an observation which agrees with previous reports (Davison et al., 1981b). Glomerular filtration rate is determined by three factors: 1. the hydrostatic and oncotic pressure gradients across the glomerular membrane, 2. the glomerular plasma flow and 3. the glomerular permeability (Moe et al., 1991). Only the first two factors are known to change markedly in early pregnancy. Whether the decrease in plasma albumin and with it, the decrease in oncotic pressurt leads to a higher glomerular filtration rate is not clear. The plateauing of glomerular filtration rate after the first trimester in spite of a continued decline in serum albumin opposes an important role of hemodilution in the pregnancy-related rise in glomerular filtration rate. Several investigators have demonstrated that the early rise in glomerular filtration rate was paralleled by a rise in effective renal plasma flow (Sims and Krantz, 1958; Dunlop 1980). The larger fractional rise in effective renal plasma flow relative to that in glomerular filtration rate suggests that a primary increase in effective renal plasma flow may have triggered the rise in glomerular filtration rate (Deen et al., 1972; Dunlop 1980). However, experimental prove for such a causal relationship should come from a study in which effective renal plasma flow and glomerular filtration rate are measured together between conception and the eighth week of pregnancy. The higher 24-hour creatinine clearance in early pregnancy is paralleled by a lower fractional salt excretion, probably indicating intrarenal compensation to minimize the urinary sodium wastage associated with accelerated sodium filtration.

Early pregnancy changes in steroid environment in our study included an increase in 17ß-estradiol, a decrease in 17-hydroxyprogesterone and little change in progesterone. These changes are consistent with observations reported by others (Exalto 1979). Because the levels of progesterone changed little in early pregnancy, its possible causal role in the early hemodynamic adaptations is doubtful. On the other hand, neither the increase in 17ß-estradiol nor the decrease in 17-hydroxyprogesterone correlated with the observed changes in hemodynamics, 24-hour creatinine clearance or related volume parameters, possibly because the largest change may have occurred before our first measurement. 
Therefore a possible role of these steroids requires study in the first 3 weeks after conception.

On the basis of the results from this study we hypothesize that in early pregnancy a not yet identified endocrine stimulus induces an overall decrease in vascular tone, leading to a systemic vasodilatation and a rise in arterial compliance, with both factors independently contributing to a fall in afterload. Stroke volume increases in response to this afterload reduction. The reduced tone of the vascular wall, together with the vasodilatation, may be directly responsible for both down-regulation of the baroreceptor and the nonosmotically-induced release of vasopressin (Schrier and Briner, 1991). The latter leads to hemodilution and with it a reduction in blood viscosity. A lower blood viscosity potentiates the fall in vascular resistance. These changes combined are responsible for the realization of a high flow-low resistance circulation in early pregnancy that enables the cardiac output to be chronically increased without requiring extra cardiac work (cardiac output times MAP). The rise in 24-hour creatinine clearance in concert with the rise in cardiac output results from the vascular relaxation of afferent and efferent renal vessels. The rise in left atrial diameter and the fall in plasma renin concentration between the fifth and eighth week and the plateauing of the 24-hour creatinine clearance after the eighth week support a rise in vascular filling state, probably as a compensation for an initial state of hypovolemia. The lack of changes in the inferior vena cava dimensions may indicate that the postulated transient mismatch between size and filling state of the vascular bed develops predominantly in arteries because of their higher smooth muscle content. Consequently, changes in the inferior vena cava may have been too small to be detectable with this technique.

Although this hypothesis requires additional experimental prove, the results of this study provide strong evidence that the changes in volume homeostasis and renal function in early pregnancy develop mostly secondary to the adaptive changes in maternal hemodynamics, rather than independently in response to the same or a different stimulus. These findings also emphasize that the most important maternal adaptations to pregnancy take place in the first 8 weeks.

\subsection{Acknowledgements}

This study was financially supported by Schering Nederland BV. 
Chapter 7

\section{SEVERELY IMPAIRED FETAL GROWTH IS PRECEDED BY MATERNAL HEMODYNAMIC MALADAPTATION IN VERY EARLY PREGNANCY}

Johannes J Duvekot MD ${ }^{1}$, Emile C Cheriex $\mathrm{MD}^{2}$, Frans AA Pieters $\mathrm{MD}^{2}$, and Louis LH Peeters MD, PhD ${ }^{1}$

From the Departments of Obstetrics and Gynecology ${ }^{1}$ and Cardiology ${ }^{2}$, University Hospital Maastricht, PO Box 1918, 6214 BX Maastricht, The Netherlands.

Submitted for publication 


\subsection{Abstract}

Objective:

\section{Setting:}

Subjects:

Methods:

Results:

Conclusion:
To test the hypothesis that in pregnancies complicated by intrauterine growth retardation (IUGR) maternal cardiovascular adaptation is already abnormal in the first weeks of pregnancy.

University Hospital Maastricht, Maastricht, the Netherlands.

Fourteen healthy pregnant women, recruited from the subfertility clinic. Ten pregnancies were uneventful and four pregnancies resulted in the birth of growth retarded infants.

Maternal cardiovascular status was followed longitudinally by combined M-mode and Doppler echocardiography. Studies were performed weekly between the fifth and 10th week, at 14, 25 and 35 weeks and postpartum. Differences between the two groups were analyzed by nonparametric tests.

In early pregnancy, the IUGR group differed from the normal group by a consistently smaller and not increasing left atrial diameter and a cardiac output that failed to increase. Postpartum the subjects in the IUGR group had a significantly smaller left atrial diameter and faster mean circumferential fibre shortening.

Maternal hemodynamic adaptation in the first weeks of pregnancy is defective in IUGR pregnancies, presumably associated with a concomitant inadequacy of the vascular filling state. 


\subsection{Introduction}

In pregnancy the clinical expression of both intrauterine growth retardation (IUGR) and hypertensive disorders is characterized by a long latent phase. It is assumed that these conditions are triggered by a defective interaction between trophoblast and uterine tissues resulting in so-called suboptimal placentation in the first half of pregnancy (Khong et al., 1986). Our knowledge about this subclinical period in IUGR is only fragmentary. It is still obscure as to whether the defective trophoblast invasion and maternal maladaptation are triggered by a common, yet unidentified factor or, alternatively, whether abnormal trophoblast development results in poor placentation and subsequently decompensation of initially normally developing maternal adaptation to pregnancy (Roberts et al., 1989; Taylor et al., 1990; Roberts et al., 1991; Cunningham and Lindheimer, 1992). Systematic exploration of the initial patterns of change in a broad spectrum of cardiovascular variables, in pregnancies eventually complicated by IUGR, can be expected to provide valuable indirect information about the pathogenesis of IUGR.

The results of a previous study in early uneventful pregnancy (Duvekot et al., 1993a), suggested a rather abrupt expansion of the intravascular compartment in the first 2-3 weeks after implantation, most likely secondary to systemic vasodilation. This condition is associated with a fall in both afterload and vascular filling pressure, giving rise to an increase in cardiac output and activation of volume conserving mechanisms, respectively (Schrier and Briner, 1991; Duvekot et al., 1993a) By the eighth pregnancy week, volume retention appears to have resulted in the correction of the relative hypovolemia. However, the initially instituted high flow/low resistance circulation persists.

The present study was designed to test the hypothesis that in pregnancies complicated by severe IUGR, pregnancy is already abnormal during or shortly after implantation, giving rise to deficient placentation as well as early maternal hemodynamic maladaptation. To this end, we studied serially the maternal cardiovascular adaptation in early pregnancy in four subjects which subsequently developed severe IUGR. The results were compared with those previously reported on 10 subjects with uneventful pregnancies (Duvekot et al., 1993a). 


\subsection{Subjects and Methods}

Selection and characteristics of subjects: Twenty-two, healthy, normotensive Caucasian subjects, younger than 40 years of age were recruited in consecutive order from our subfertility clinic as soon as pregnancy was established. After confirmation of pregnancy by a sensitive urinary human chorionic gonadotropin determination and after receiving informed consent, the women were enrolled in this study. Conceptional dates were either based on ultrasonic ovulation-detection or were calculated on the basis of an early crown-rump length measurement. The amenorrhea (in weeks) was standardized by adding 2 weeks to the conceptional date.

Eight subjects aborted spontaneously before the tenth week. The course of ten (singleton) pregnancies was uneventful. All subjects in this group delivered of healthy appropriately grown infants with birth weights ranging from the tenth to the 90th centile of the reference population (Voorhorst et al., 1990). Four nulliparous subjects developed fetal growth retardation during late pregnancy. The newborns in this group weighed less than the tenth centile and three of these infants had birth weights more than two standard deviations below the mean. Clinical assessment indicated that the growth impairment did not result from previous intrauterine infection or from congenital anomalies. For the purpose of this prospective study the data obtained during the ten uneventful pregnancies were compared with those obtained in the four pregnancies complicated by fetal growth retardation. The subjects' characteristics are listed in Table 1.

Table I Characteristics of Subjects with Normal and Patbological Pregnancies

\begin{tabular}{|c|c|c|c|}
\hline & $\begin{array}{l}\text { normal pregnancies } \\
(\mathrm{n}=10)\end{array}$ & $\begin{array}{l}\text { pathological pregnancies } \\
(n=4)\end{array}$ & P-value@ \\
\hline Maternal age (years) & $29.1(23-34)$ & $27.3(22-32)$ & ns \\
\hline Maternal height $(\mathrm{cm})$ & $167(154-175)$ & $169(167-173)$ & ns \\
\hline Maternal prepregnancy weight $(\mathrm{kg})$ & $65.9(52.5-82)$ & $62.0(49-80)$ & ns \\
\hline Incidence nulliparity & 7 & 4 & ns* \\
\hline Gestational age at birth (wks) & $40.0(38.3-40.6)$ & $36.4(28-40.3)$ & ns \\
\hline Newborn weight $(\mathrm{g})$ & $3411(2870-3990)$ & $1960(750-2540)$ & $<0.005$ \\
\hline
\end{tabular}

NS - not stgnificant.

Data are presented as medians witb tbe range in parentbeses.

a Mann-Wbitney U-test.

- Fisber exact test.

Initial hemodynamic function was normal in all subjects, as indicated by normal electrocardiography and echocardiography at first study at five weeks' amenorrhea (35 \pm 3 days). Studies were repeated at weekly intervals between the fifth and tenth weeks, at 14, 25 and 35 weeks of pregnancy and either 3 months post partum or 3 months after discontinuation of breastfeeding. Each study session consisted of echocardiography and the measurement of blood pressure. 
Echocardiographic methods: The echocardiographic recordings were made with a cross-sectional, phased array echocardiographic Doppler system (Hewlett-Packard Sonos 500 and 1000). This system utilizes a $2.5 \mathrm{mHz}$ phased array transducer with continuous-wave and a $1.9 \mathrm{mHz}$ independent continuous-wave transducer. Data were registered on a strip chart recorder using a paper speed of $25 \mathrm{~mm} \cdot \mathrm{s}^{-1}$ for Mmode -, and $50 \mathrm{~mm} \cdot \mathrm{s}^{-1}$ for Doppler measurements. Standard echocardiographic views were stored on videotape. All measurements were performed in semi-left lateral position after about $5 \mathrm{~min}$ acclimatization to the experimental set-up. M-mode measurements were performed according to the recommendations of the American Society of Echocardiography (Sahn et al., 1978). The methodology of the measurements and calculations were described previously (Duvekot et al., 1993a).

At the end of the study, systolic and diastolic blood pressure were measured using a cuff sphygomanometer. Diastolic blood pressure was taken at Korotkoff phase IV. Echocardiographic and Doppler measurements and the subsequent blood pressure measurements were all performed at random by two experienced cardiologists unaware of the subjects' gestational age (co-authors ECC and FAAP).

Statistical methods: The characteristics between the two groups were compared by Mann-Whitney U-test or Fisher exact test where appropriate (Table 1). Because of the modest number of subjects in each group we chose to use the Mann-Whitney U test to compare the variables determined in the "pathological" group complicated by fetal growth retardation with those in the "normal" group. Since the greatest changes in most variables were noted to occur between the fifth and eighth weeks (Duvekot et al., 1993a), comparisons were only performed for this period. For each of the ten normal and four IUGR pregnancies, (linear) regression lines were computed for the relationships between the measured variables (dependent) and gestational age between 5 and 8 weeks (independent). By this approach the four intra-individual data points for each variable were summarized as recommended for the analysis of serial measurements (Matthews et al., 1990). This calculation yielded 14 individual slopes for each of the variables studied. Then, the two samples of slopes obtained for each variable were comparable using the Mann-Whitney U test. Furthermore, the early-pregnancy means for the period between the fifth and eighth weeks were calculated for each individual, and then compared between the two groups (Matthews et al., 1990). The values obtained after the eight week were weekly compared between the two groups. Finally, the changes, from early-pregnancy mean to corresponding postpartum value, were compared between the two groups, also using the Mann-Whitney U test. Throughout the text, data are presented as medians with ranges in parentheses, unless otherwise stated. P less than $5 \%$ was considered significant. 


\subsection{Results}

The medians determined for both groups for the weekly measured heart rate, stroke volume and cardiac output (figure 1) and the median slopes for the rate of change in these variables between the fifth and eighth pregnancy week are listed in Table II. Although the early-pregnancy means for heart rate, stroke volume and cardiac output did not differ between the two groups, cardiac output increased more in the normal group.

Figure 1 Cardiac output during first trimester in normal (open bars) and patbological pregnancies (closed bars). Error bars $=$ upper range.

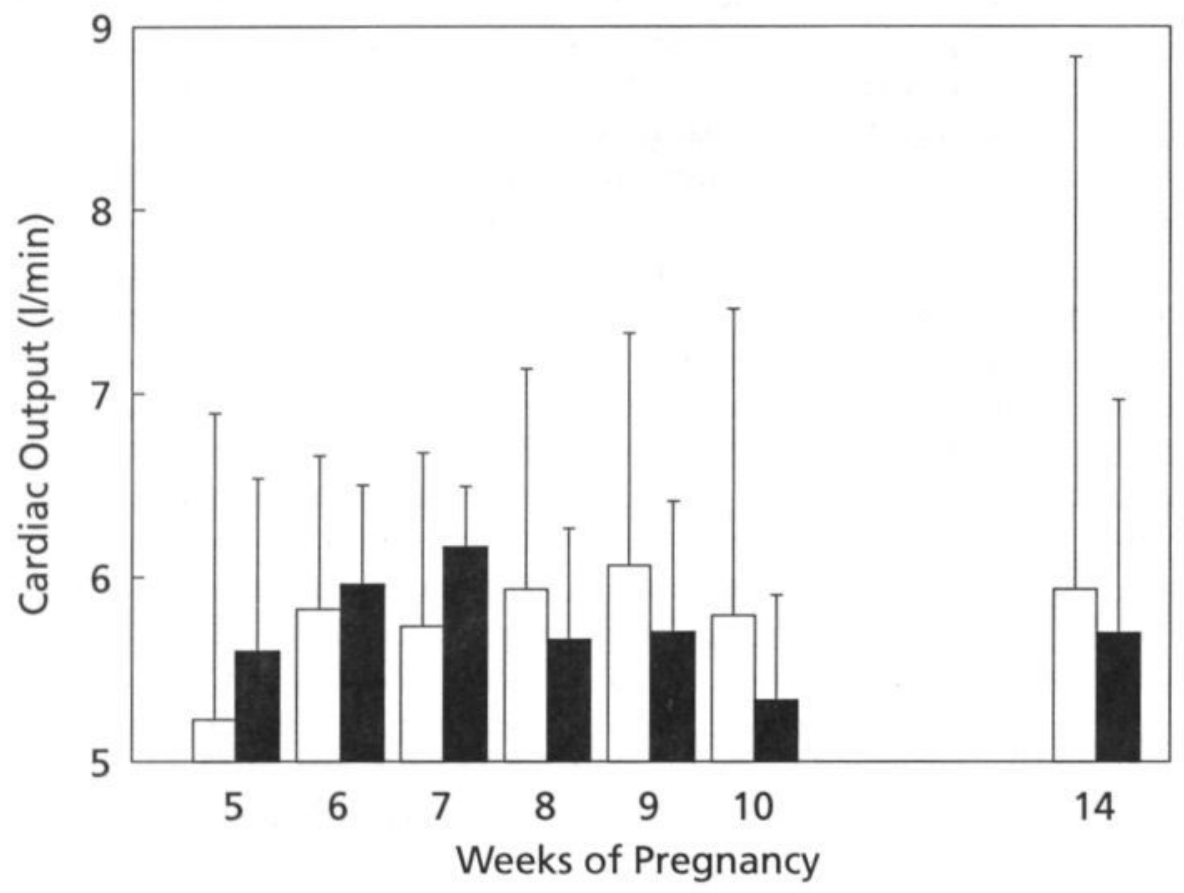



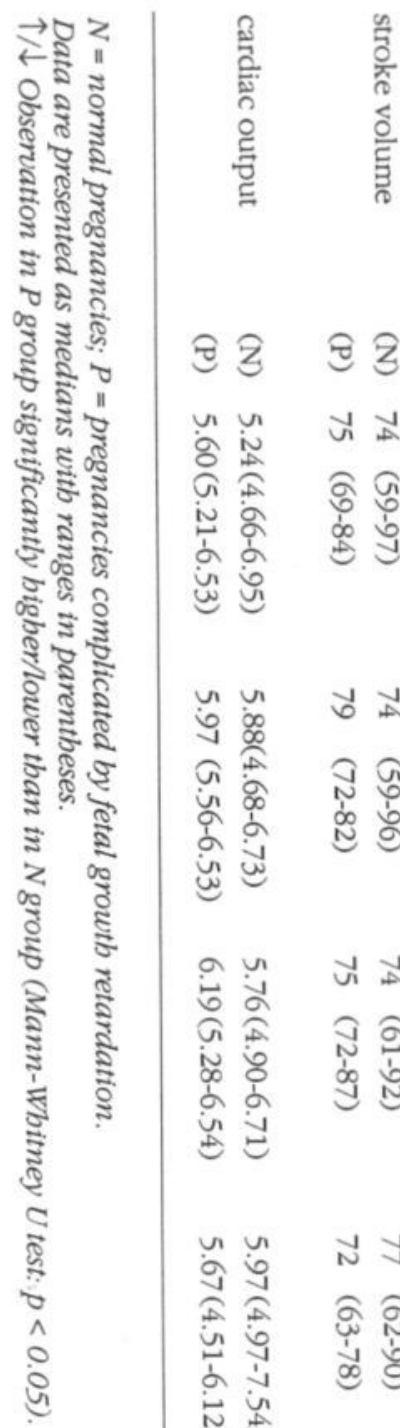

ఏ

$\frac{2}{2}$

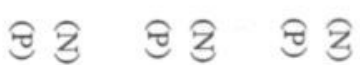

บับ ปั

8 송

자 तิ

సà d

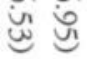

$\forall$

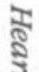

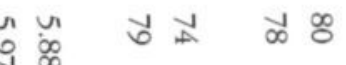
ง

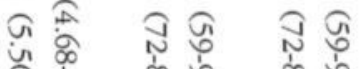

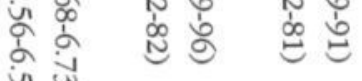

జ్ల జ్ర

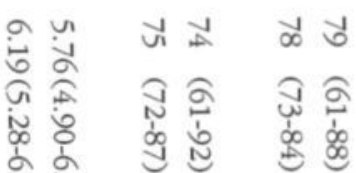

过

บับ

조

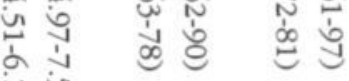

总芯

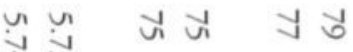

म.

สิ ปิ

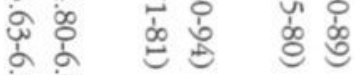

जิ

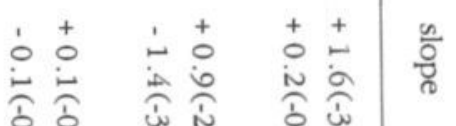

i 0 in

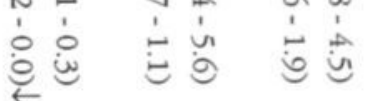


The median values and median rates of change between the fifth and eighth week, calculated for the indices for preload (left atrial diameter (figure 2), left ventricular end-diastolic dimension), myocardial contractility (mean velocity of circumferential fibre shortening, left ventricular mass) and afterload (mean arterial pressure, total peripheral vascular resistance) are presented in Table III. Of the values listed, only left atrial diameter was smaller and increased less in the IUGR group.

The data obtained after the eighth week are listed in Tables IV and V. Second and third trimester heart rate, stroke volume, cardiac output, mean arterial pressure and total peripheral vascular resistance did not differ between the two groups. The smaller left atrial diameter in the IUGR group persisted throughout pregnancy and was, in the second half of pregnancy, accompanied by a smaller left ventricular enddiastolic dimension and left ventricular mass. Comparison of the postpartum values between both groups indicated a smaller left atrial diameter and a faster mean velocity of circumferential fibre shortening in the IUGR group.

The mean early-pregnancy - and postpartum data in the two groups are listed in Table VI. Because of the small sample size in the IUGR group, the within-group comparison by Wilcoxon Signed Rank Test could only be performed in the normal group. By this analysis it was demonstrated that heart rate, cardiac output and mean velocity of circumferential fibre shortening were consistently higher, and total peripheral vascular resistance consistently lower in early normal pregnancy relative to postpartum. Interestingly we observed in the IUGR group corresponding consistency for these variables except for velocity of circumferential fibre shortening.

Figure 2 Left atrial diameter during first trimester in normal (open bars) and patbological pregnancies (closed bars). Error bars $=$ upper range.

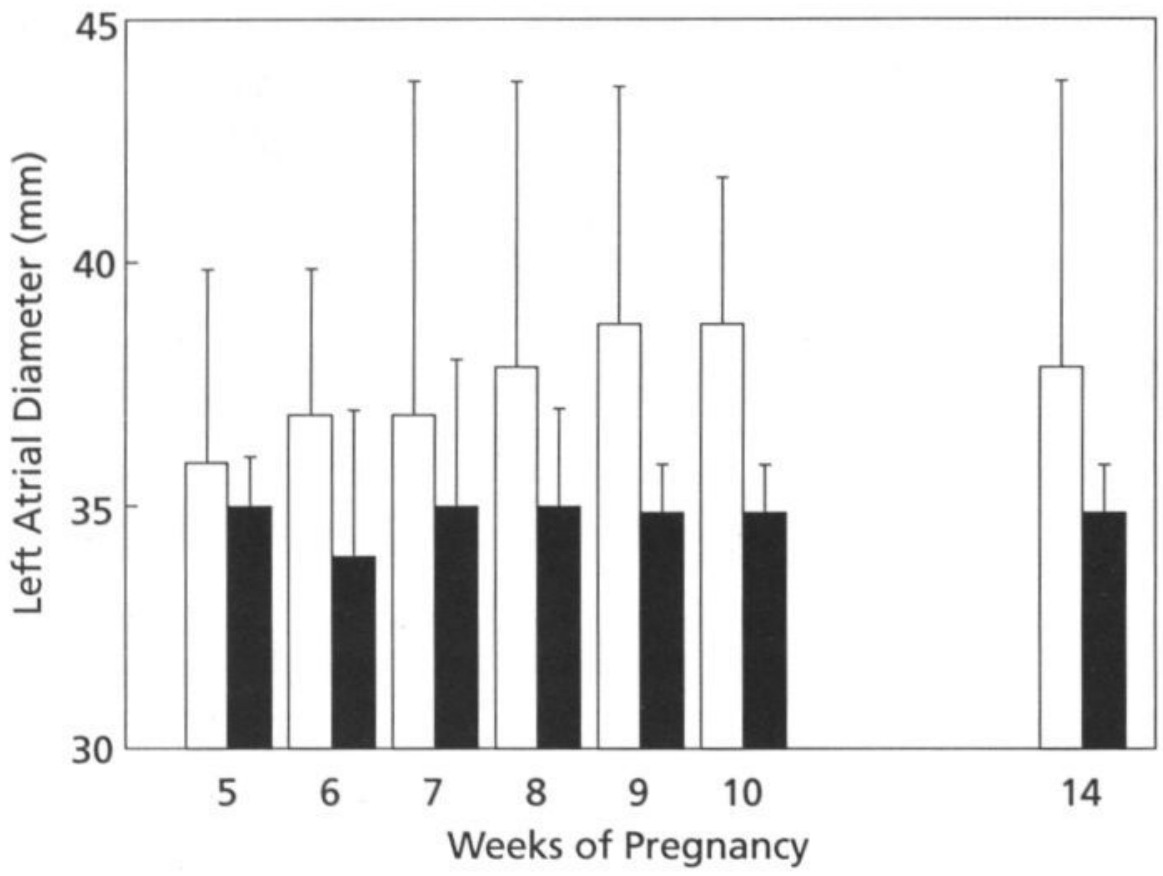




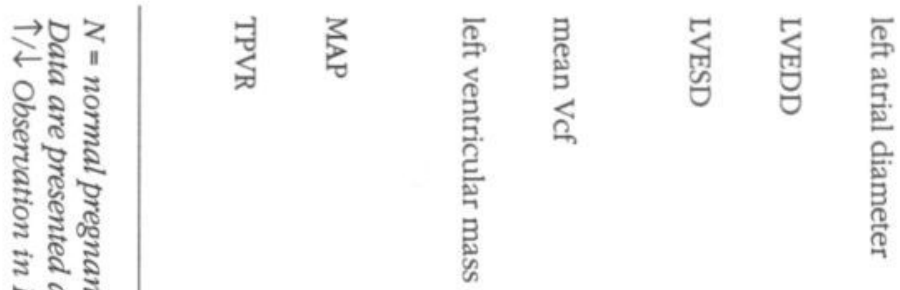

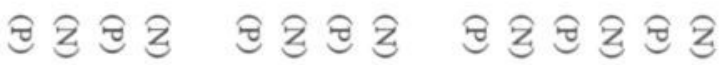

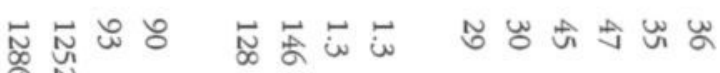

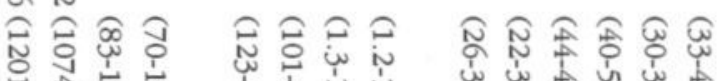

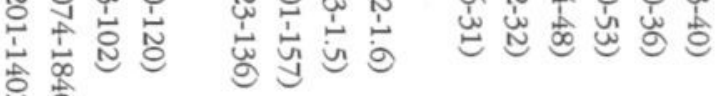
ฐ ริ) 20 s.

कำ

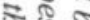

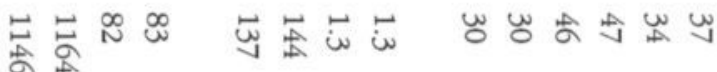

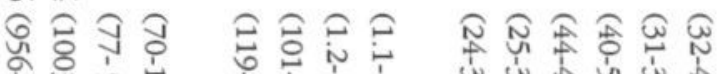

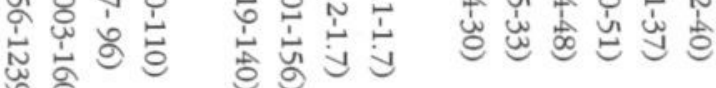

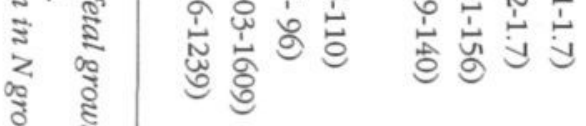

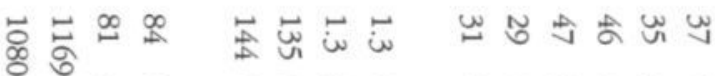

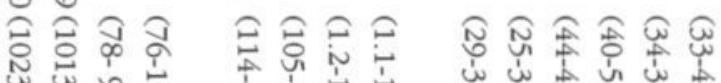

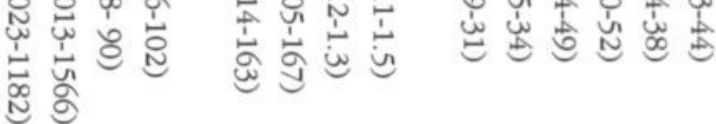

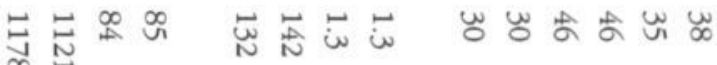
क 0 -

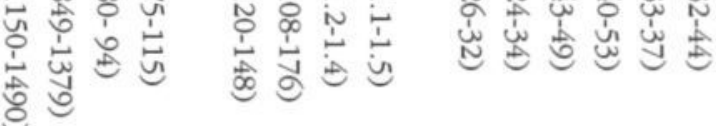

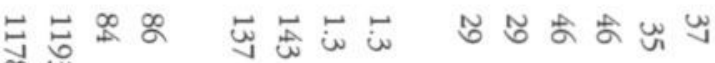

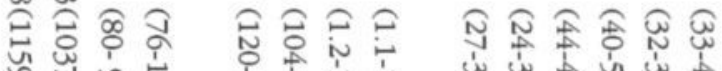

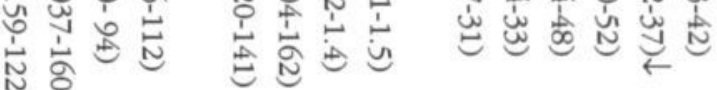
柋

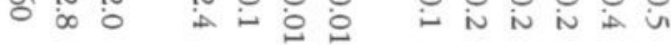

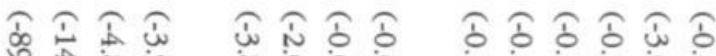
車 त्रे

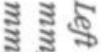
ख्व เ a 고 응 공 호요 क् 궁 ป స్ ㅋํำ ํㅗㄹ

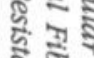
ริ कू 구원 त्र की० ฐ 2 ำ 궁

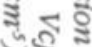
구 볼 총 ज्ञ สำ 施 ไี้ ฐิ 공 กิ 곡 궁 ถู

อำ 


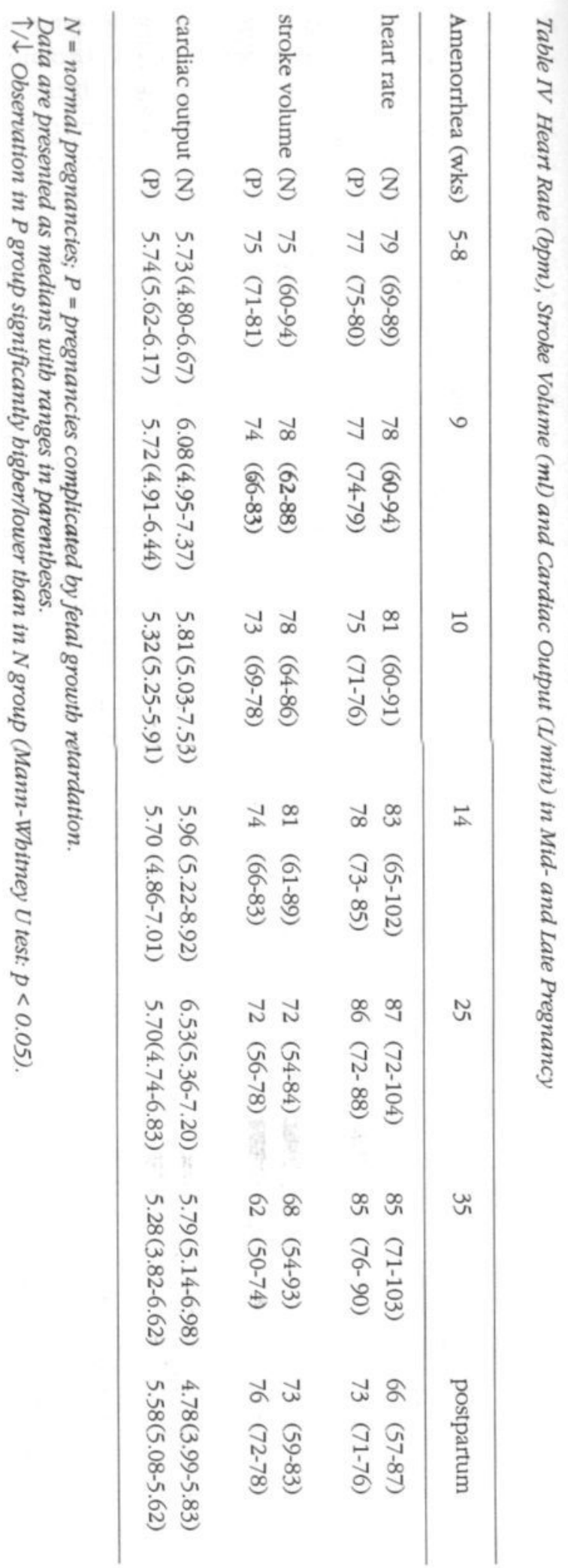




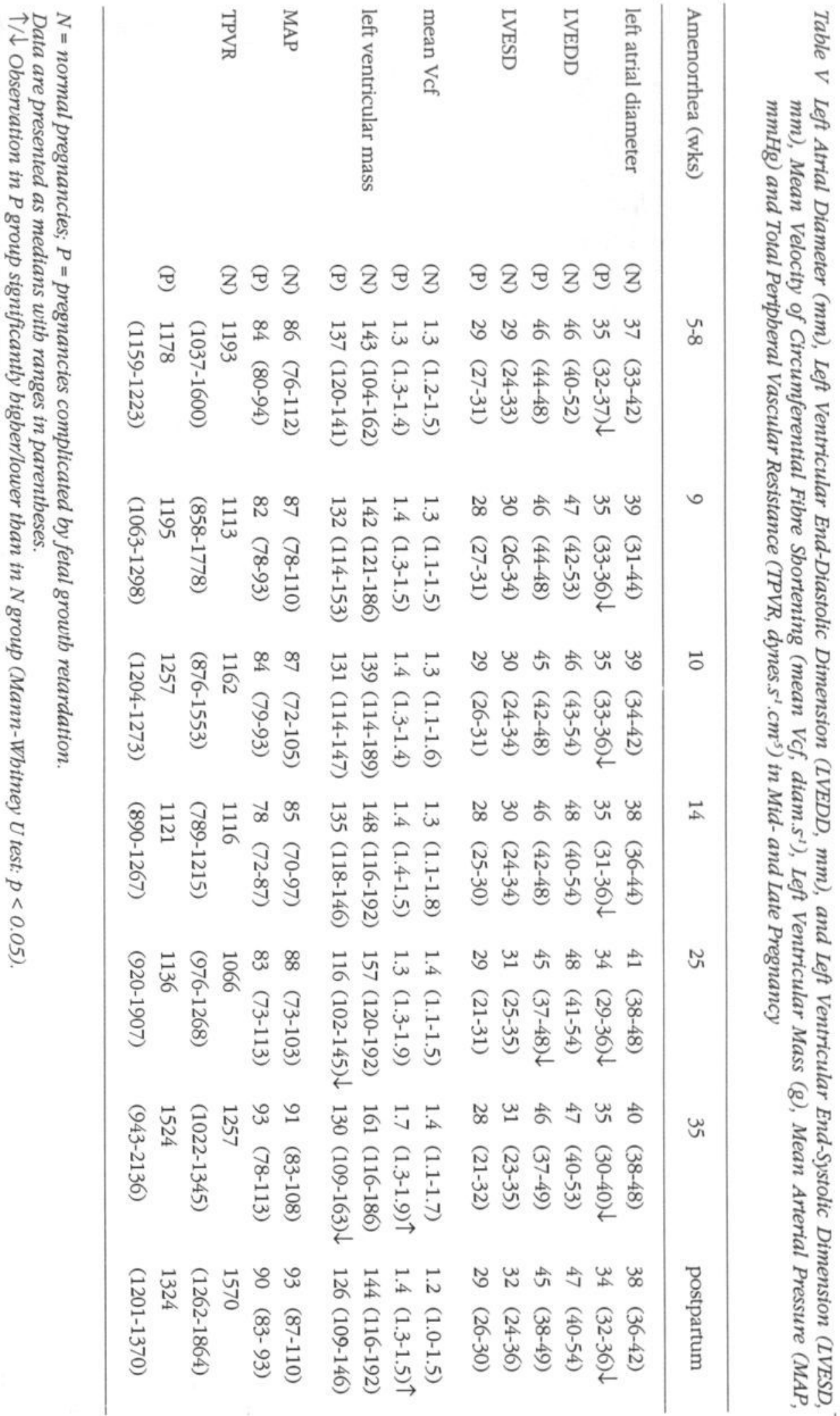




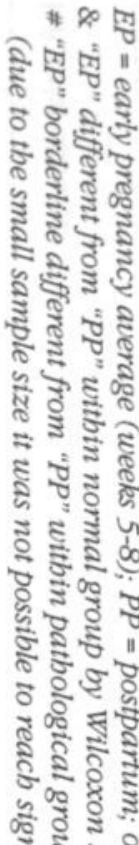

\% 웡

实高

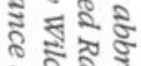

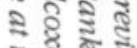

c.

$\Delta$ \%

0 ॠ 1 के

i $\begin{aligned} & 0 \\ & 0\end{aligned}$

ฐ

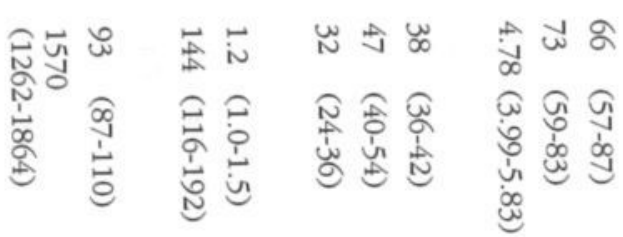

氙芯总

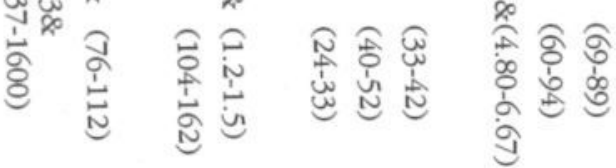

ब

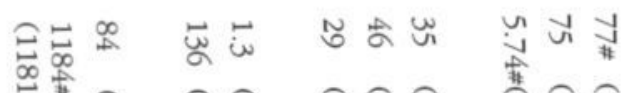

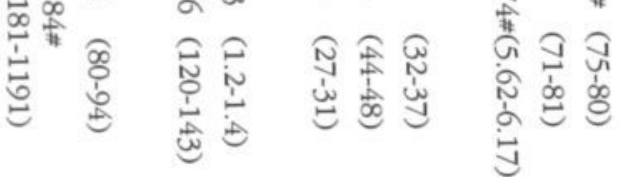

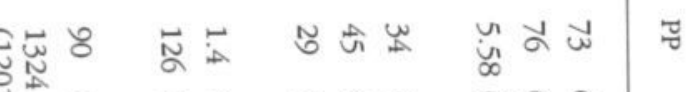

8

"

कू

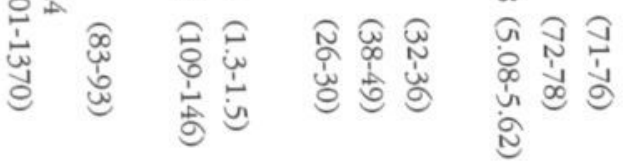




\subsection{Discussion}

The present study was designed to test the hypothesis that in pregnancies complicated by IUGR, the subclinical period does not begin with poor placentation between 14 and 18 weeks giving rise to decompensation of an initially normally adapted hemodynamic function, but rather begins at or shortly after implantation as a trophoblastic disorder which leads to both maternal hemodynamic maladaptation and defective placentation. In order to test this hypothesis, we determined the earlypregnant patterns of changes in a number of relevant hemodynamic variables in four subjects which developed severe IUGR in late pregnancy. The results were compared with those obtained in a group of women with uneventful pregnancies (Duvekot et al., 1993a). The high incidence of IUGR (4/22) and miscarriage (8/22) encountered in the subjects recruited for this study was explained as being related to the well-known poor reproductive performance of subfertile subjects frequently conceiving in hormonally manipulated cycles (Williams et al., 1991; Coulam 1992). An uncomplicated course of pregnancy resulting in the birth of a healthy appropriately grown infant, assured in our view a normal maternal adaptation, irrespective of the initial cause of the subfertility. By choosing nonparametric tests and evaluating changes in a wide variety of complementary variables it was possible to discern differences between normal and pathological pregnancies with valid statistical methods, in spite of the limited group sizes. So far, no other study evaluated data, obtained prospectively, longitudinally and in early pregnancy, between two groups as in the present study.

In our study the noninvasive method of combined M-mode and continuous wave Doppler echocardiography was chosen to evaluate maternal cardiovascular function. Also for the pregnant state, this method has been well validated using classical invasive techniques as a reference and was found to be associated with a type II error of less than 5\% (Easterling et al., 1987). Doppler flow measurements were performed at the level of the aortic valve, a measurement site yielding results that correlated better with concomitantly obtained reference data than those generated at the level of the mitral or pulmonary valve (Robson et al., 1987d). To our knowledge, no studies have been reported describing the patterns of change in early-first-trimester hemodynamics in pregnancies complicated by IUGR in advanced pregnancy. Already between the fifth and eighth week, the IUGR group differed from the reference group by a failure of the cardiac output and left atrial diameter to increase and by a consistently smaller left atrial diameter. The latter phenomenon is in accordance with a state of inadequate vascular filling (Di Donato et al., 1982; Duvekot et al., 1994a). In this presumption the concomitant decrease in mean velocity of circumferential fibre shortening can be interpreted as a weakening of an initially raised cardiac contractility, a compensatory mechanism unsuitable to maintain cardiac output for a prolonged period. On the basis of these considerations, we explain the observed set of changes between 5 and 8 weeks as follows. The initial fall in mean circulatory filling pressure results in a relative hypovolemia and with it a fall in preload and thus venous return. At first the latter is compensated for by a rise in heart rate and cardiac contractility. Since these 2 mechanisms are only designed to provide acute and temporary compensation for cardiac output insufficiency, their supporting effect 
on cardiac output will fade over time resulting in a gradual decline in stroke volume. Also after the eighth week, the smaller dimensions of the left atrium and to a lesser extent the left ventricle persist in the IUGR group, which supports persisting chronic inadequacy of the vascular filling state throughout pregnancy. This phenomenon is in agreement with previous reports using various different techniques (Hytten and Paintin, 1963; Gibson 1973; Goodlin et al., 1981).

Although we did not perform hemodynamic measurements during the first 3 weeks after conception, the postpartum data may have some value as nonpregnant reference value (Capeless and Clapp, 1991; Robson and Dunlop, 1992). As compared to the normal group, the postpartum left atrial diameter was smaller and the mean velocity of circumferential fibre shortening was faster (Table VI), in the IUGR subjects. In contrast to the hemodynamic function in a reference population, these subjects seem to have a marginally filled vascular bed and with it a lower venous return. To maintain cardiac output these subjects seem to have a relatively increased cardiac contractility. The circulation is characterized by a high cardiac output for the given amount of circulating volume/venous return which can be defined as a "hyperdynamic" circulation. Such a circulatory function has also been observed postpartum in previously preeclamptic patients (Easterling et al., 1990c). In the latter study cardiac output was also elevated and total peripheral vascular resistance reduced resembling the trends observed in the comparison between the two groups in our study. It is tempting to speculate that a preexistent hyperdynamic circulation is hampered by a reduced reserve capacity to withstand a transient period of physiologic hypovolemia. The persisting hypovolemia in IUGR pregnancy is likely to lead to circulatory insufficiency which can be expected to raise sympathetic tone and to interfere with placental development. In animal studies it has been demonstrated that an initially reduced uterine blood flow interferes with placentation and may result in IUGR (Creasy et al., 1972). Interestingly, three decades ago Scandinavian investigators already suggested that women with a smaller heart volume had an increased risk to deliver of SGA infants (Hedberg and Radberg, 1962).

In conclusion our observations provide evidence for a defective maternal hemodynamic adaptation in the first weeks of pregnancies, which are complicated by IUGR in late pregnancy. This maladaptation consists of a diminished response to the presumed initial generalized vascular relaxation which is kept responsible for an early relative underfill state. Postpartum the maternal circulation appears to remain at least for 3 months "hyperdynamic". Whether a preexisting "hyperdynamic" circulation predisposes for later IUGR requires study of the circulatory function starting antenatally.

\subsection{Acknowledgments}

This study was sponsored by Schering Nederland BV, Weesp, the Netherlands. 


\section{Chapter 8}

\section{MATERNAL VOLUME HOMEOSTASIS IN EARLY PREGNANCY IN RELATION TO FETAL GROWTH RETARDATION}

Johannes J Duvekot MD', Emile C Cheriex MD², Frans AA Pieters $\mathrm{MD}^{2}$, Paul PCA Menheere $\mathrm{PhD}^{3}$ and Louis $\mathrm{LH}$ Peeters MD, $\mathrm{PhD}^{1}$

From the Departments of Obstetrics and Gynecology ${ }^{1}$, Cardiology ${ }^{2}$ and Clinical Chemistry $^{3}$, University Hospital Maastricht, PO Box 1918, 6214 BX Maastricht, The Netherlands.

Obstetrics \& Gynecology 1995; in press. 


\subsection{Abstract}

Objective:

Methods:

Results:

\section{Conclusions:}

To test the hypothesis that the volume adaptation in pregnancies complicated by fetal growth retardation is already abnormal in very early pregnancy.

The volume homeostasis in the first 8 gestational weeks, in six pregnancies complicated by fetal growth retardation was compared to that in ten normal pregnancies. Creatinine clearance, volume-dependent hormones, hemodilution-related variables, and ultrasonic cardiovascular dimensions were measured weekly between the fifth and tenth week, in the second and third trimesters, and postpartum. Differences between the two groups were analyzed by nonparametric tests.

In very early pregnancy, pregnancies complicated by fetal growth retardation differed from normal pregnancies by a smaller left atrial diameter, a smaller collapsible part of the inferior vena cava, a smaller fall in serum creatinine and urea, and a lower serum sodium concentration.

Pregnancies subsequently complicated by fetal growth retardation are preceded by inadequate volume adaptation in the initial 8 weeks. As a consequence, the transient state of relative vascular underfill seen in this period in normal gestation, persists beyond the eighth week. 


\subsection{Introduction}

In early human pregnancy mean arterial pressure falls and cardiac output and plasma volume rise compared to the prepregnant state (Duvekot et al., 1993a). These changes begin to develop before the eighth pregnancy week and may be triggered by an initial fall in vascular filling (Phippard et al., 1986; Schrier and Briner 1991; Duvekot et al., 1993a). The early rise in cardiac output and fall in blood pressure is accompanied by a rise in renal perfusion and glomerular filtration rate (Davison and Noble, 1981a; Duvekot et al., 1993a). In spite of the sodium wasting effect of the increased glomerular filtration rate, plasma volume begins to increase in this period, reaching a plateau of $1250 \mathrm{~mL}$ above nonpregnant levels in the third trimester (Hytten and Paintin, 1963; Whittaker and Lind, 1993). The importance of these hemodynamic adjustments to pregnancy is indicated by the high incidence of inadequate plasma volume expansion in pregnancies complicated by fetal growth retardation (Gibson 1973; Goodlin et al., 1981). Nevertheless, it is still unclear whether the volume adjustments in these pregnancies develop abnormally from the beginning or deteriorate after the first trimester, as has been reported for pregnancies complicated by preeclampsia (Gallery et al., 1975).

In a recent echocardiographic study we found differences in the initial hemodynamic adjustments between normal pregnancies and pregnancies complicated by fetal growth retardation (Duvekot et al., 1992). On the basis of these observations we postulated that blood volume expansion in the first weeks of pregnancies destined to produce growth restricted fetuses is inadequate to balance the initial state of vascular underfill, a transient physiologic condition occurring in the first weeks of normal human pregnancy (Duvekot et al., 1993a). As a consequence, the initial relative hypovolemia persists throughout pregnancy. To test this hypothesis, the early-pregnancy patterns of change in variables related to volume homeostasis in six pregnancies later on complicated by fetal growth retardation were compared with those in ten pregnancies with an uneventful course.

\subsection{Subjects and Methods}

Twenty-four, healthy, normotensive Caucasian women were recruited in consecutive order from our subfertility clinic as soon as pregnancy was established. Conceptional dates were either based on ultrasonic ovulation detection or calculated on the basis of an early crown-rump length measurement. The amenorrhea (in weeks) was standardized by adding two weeks to the conception date. For reasons of medical ethics, plasma volume measurements were not performed.

Eight subjects aborted spontaneously before the tenth week. The course of ten other (singleton) pregnancies was uneventful, producing healthy appropriately grown infants with birth weights ranging from the tenth to the 90 th centile of the reference population (Voorhorst et al., 1990). Six nulliparous subjects developed fetal growth retardation during late pregnancy. The newborns in this group weighed less than the tenth centile and five of these infants had birth weights more than two stan- 
dard deviations below the mean. Clinical assessment indicated that the growth impairment did not result from previous intrauterine infection or from congenital anomalies. The data obtained during the ten uneventful pregnancies were compared with those obtained in the six pregnancies complicated by fetal growth retardation. The subjects' characteristics are listed in Table 1 . Initial hemodynamic function was normal in all subjects, as indicated by normal electrocardiography and echocardiography at first study at 5 weeks' amenorrhea ( $35 \pm 3$ days). Studies were repeated at weekly intervals between the fifth and tenth weeks, at 14, 25 and 35 weeks of pregnancy and either 3 months post partum or 3 months after discontinuation of breastfeeding. Each study session consisted of blood sampling, echocardiography, and the measurement of blood pressure. In the 24 hours following each session, urine was collected in order to assess creatinine and sodium excretion.

Before ultrasonography, blood samples were withdrawn from the antecubital vein in the sitting position after approximately 5 minutes of rest. All samples were collected between 9 and 10 a.m. Blood samples for the measurement of electrolytes and proteins were withdrawn in $10 \mathrm{~mL}$ Vacutainer glass tubes (Monoject; Sherwood Medical, St. Louis, Mo.) and those for the measurement of $\alpha$-atrial natriuretic peptide $(\mathrm{pmol} / \mathrm{L})$ and plasma renin concentration $(\mathrm{pg} / \mathrm{mL})$ in $5 \mathrm{~mL}$ ethylenediaminetetraacetic acid and $10 \mathrm{~mL}$ dry Vacutainer glass tubes, respectively. The latter samples were immediately put on ice and centrifuged at $4{ }^{\circ} \mathrm{C}$ for 10 minutes at $1500 \mathrm{~g}$ within 30 minutes after sampling. All blood and urine samples were stored at $-20{ }^{\circ} \mathrm{C}$ prior to assay. Serum levels of creatinine $(\mu \mathrm{mol} / \mathrm{L})$, osmolality $(\mathrm{mOsm} / \mathrm{L}), \operatorname{sodium}(\mathrm{mmol} / \mathrm{L})$, urea $(\mathrm{mmol} / \mathrm{L})$, protein $(\mathrm{g} / \mathrm{L})$ and albumin $(\mathrm{g} / \mathrm{L})$ were measured by standard chemical procedures using a Dimension Clinical Chemistry Analyzer (Du Pont Company, Wilmington, De.). Glomerular filtration rate and renal salt handling were evaluated

Table 1 Characteristics of Subjects with Normal and Abnormal Pregnancies

\begin{tabular}{|c|c|c|c|}
\hline & $\begin{array}{l}\text { normal } \\
(n=10)\end{array}$ & $\begin{array}{l}\text { abnormal } \\
(\mathrm{n}=6)\end{array}$ & P-value@ \\
\hline Maternal age (years) & $29.1(23-34)$ & $27.3(22-41)$ & ns \\
\hline Maternal height $(\mathrm{cm})$ & $167(154-175)$ & $164(160-173)$ & ns \\
\hline Maternal prepregnancy weight $(\mathrm{kg})$ & $65.9(52.5-82)$ & $62.0(49-80)$ & ns \\
\hline Incidence nulliparity & 7 & 6 & ns* \\
\hline Gestational age at birth (wks) & $40.0(38.3-40.6)$ & $38.2(28-40.3)$ & ns \\
\hline Newborn weight $(g)$ & $3411(2870-3990)$ & $2220(750-2540)$ & $<0.005$ \\
\hline Ovulation induction (clomiphene citrate) & 2 & 4 & $\mathrm{~ns}^{*}$ \\
\hline
\end{tabular}

NS $=$ not significant .

Data are presented as medians with the range in parentbeses.

(1) Mann-Wbitney $U$ test.

- Fisber exact test. 
by 24 -hour creatinine clearance $(\mathrm{mL} / \mathrm{min})$ and fractional salt excretion $(\% / 100)$. $\alpha$ Atrial natriuretic peptide, plasma renin concentration, serum 17ß-estradiol, 17-hydroxyprogesterone, and progesterone were analyzed as described previously (Duvekot et al., 1993a).

Each cardiovascular study consisted of the measurement of various cardiac indices using combined M-mode and continuous-wave Doppler echocardiography (Duvekot et al., 1993a). The results of the early adaptation of maternal hemodynamics in normal pregnancy have been reported previously (Duvekot et al., 1993a). After the echocardiographic measurements, the ultrasonic dimensions of the inferior vena cava were determined from the subxyphoidal region, with the subject in supine position. Long and short axis views of the inferior vena cava were obtained just below the diaphragm in the hepatic segment. The maximal inferior vena cava diameter (inferior vena cava diameter maximal $_{\text {) }}$ was measured during unforced expiration and before the P-wave on the electrocardiogram, to avoid interference with the A wave of the venous pressure curve. Maximal emptying of the abdominal inferior vena cava producing the smallest inferior vena cava contour (inferior vena cava diameter $_{\text {minimal }}$ ) was obtained during maximal inspiration, when venous drainage toward the thoracic inferior vena cava is maximized in response to the high negative intra-thoracic pressure. The collapsible part of the inferior vena cava was defined as

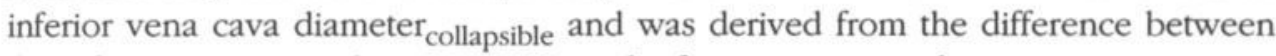

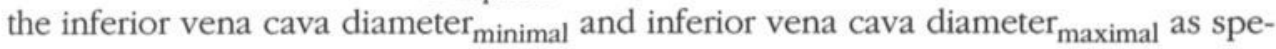
cified elsewhere (Duvekot et al., 1994b). After the ultrasonic studies, blood pressure was measured in the sitting position. Both ultrasonic studies and blood pressure measurements were performed by two experienced cardiologists, who were unaware of the subjects' gestational age (ECC and FAAP).

The characteristics between the two groups were compared by Mann-Whitney U test or Fisher exact test where appropriate (Table 1). Because of the modest number of subjects in each group we chose to use the Mann-Whitney U test to compare the variables determined in the "abnormal" group complicated by fetal growth retardation with those in the normal group. Since the greatest changes in most variables were noted to occur between the fifth and eighth weeks (Duvekot et al., 1993a), comparisons were only performed for this period. For each of the ten normal and six abnormal pregnancies, (linear) regression lines were computed for the relationships between the measured variables (dependent) and gestational age between 5 and 8 weeks (independent). By this approach the 4 intra-individual data points for each variable were summarized as recommended for the analysis of serial measurements (Matthews et al., 1990). This calculation yielded 16 individual slopes for each of the variables studied. Then, the two samples of slopes obtained for each variable were comparable using the Mann-Whitney U test. Furthermore, the early-pregnancy means for the period between the fifth and eighth weeks were calculated for each individual, and then compared between the two groups (Matthews et al., 1990). Finally, the changes, from early-pregnancy mean to corresponding postpartum value, were compared between the two groups, also using the Mann-Whitney U test. Throughout the text, data are presented as medians with ranges in parentheses, unless otherwise stated. $\mathrm{P}<0.05$ was considered significant. 


\subsection{Results}

The weekly values for serum creatinine, glomerular filtration rate and fractional salt excretion and the rate of change in these 3 variables between pregnancy weeks 5 and 8 are listed in Table 2. Although the serum creatinine concentration decreased at a slower rate in the abnormal group than in the normal group, the concomitant rate of change in glomerular filtration rate and that in fractional salt excretion were comparable in the two groups. Also the early-pregnancy mean for these 3 characteristics was comparable in the two groups.

The serum levels of the volume-dependent hormones and volume-dependent ultrasonic dimensions in early pregnancy are listed in Table 3. Although the rate of change with pregnancy differed in none of the variables between the two groups, the early-pregnancy mean for left atrial diameter and that for inferior vena cava diametercollapsible were smaller in those later complicated by fetal growth retardation.

The weekly data and early-pregnancy rate of change in the variables which vary with hemodilution are listed in Table 4. In the abnormal subjects, the serum urea decreased at a slower rate than in the normals. The rate of change in the other variables was comparable in the two groups. The early-pregnancy mean of serum sodium was lower in those complicated by fetal growth retardation.

The steroid levels in early pregnancy did not differ between the two groups (Table 5). In both groups 17ß-estradiol had increased and 17-hydroxyprogesterone had decreased at a comparable rate. The data for 17ß-estradiol increased slightly steeper in the normal group, but this did not reach significancy $(\mathrm{p}=0.051)$.

Serum creatinine, glomerular filtration rate and fractional salt excretion obtained at fourteen, 25 and 35 weeks amenorrhea did not differ between the two groups (Table 6). After the eighth week, the smaller left atrial diameter in the abnormal group persisted throughout pregnancy and was accompanied by smaller inferior vena cava dimensions at 25 and 35 weeks (Table 7). After the eighth week, none of the other variables listed in Tables 8 and 9 differed appreciably between the two groups.

The early-pregnancy mean with corresponding postpartum value for each variable in the two groups is listed in Table 10. The abnormal subjects differed by a higher postpartum fractional salt excretion and a lower postpartum serum urea than the early-pregnancy mean. Comparison of the postpartum values indicated that the two groups differed only from each other by a smaller left atrial diameter in the abnormal group. 


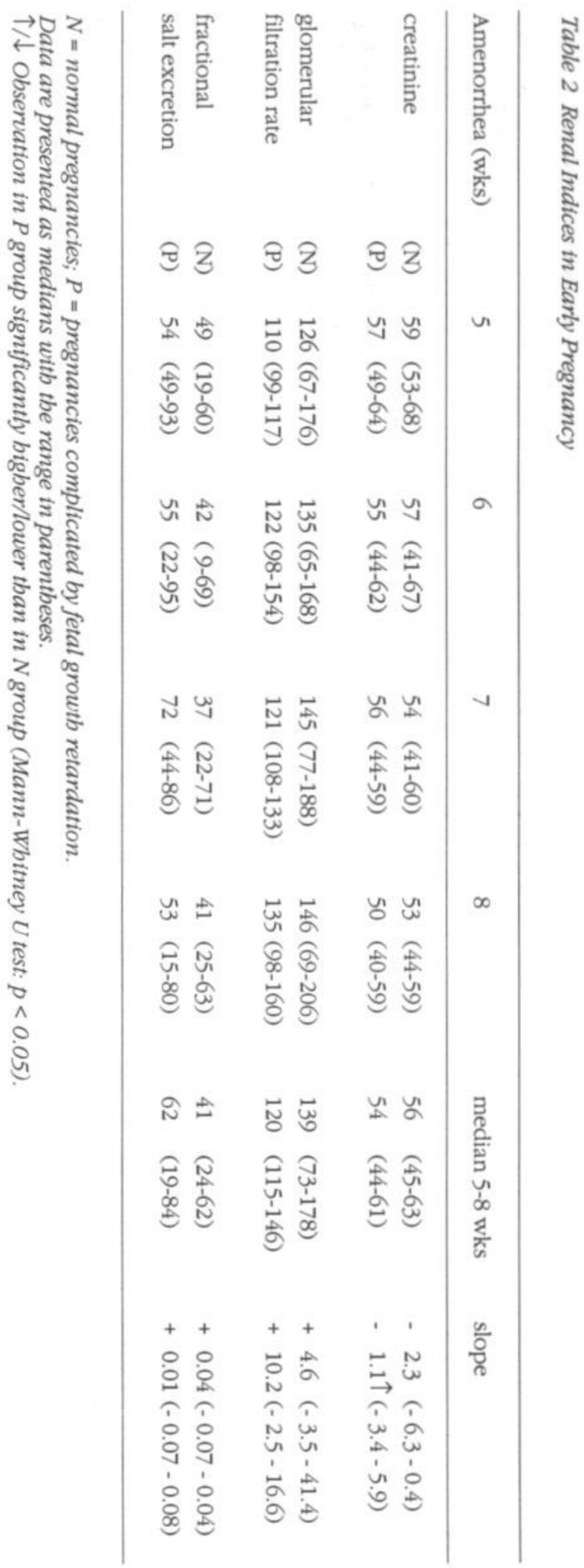




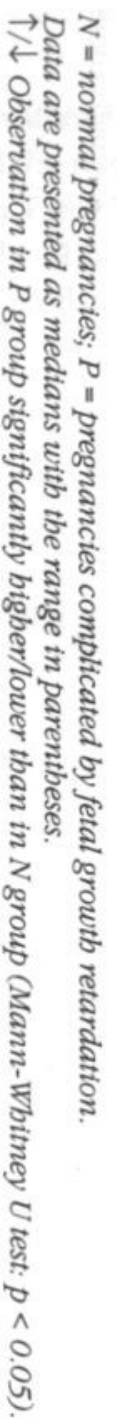

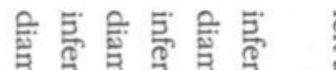

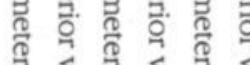 离}

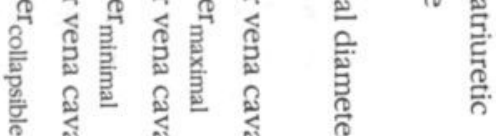

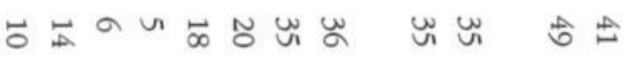
vi

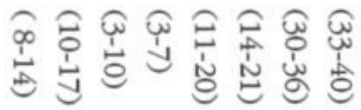
సิ

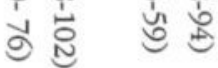
๖むの

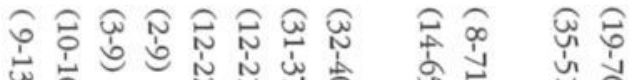

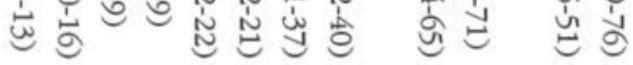

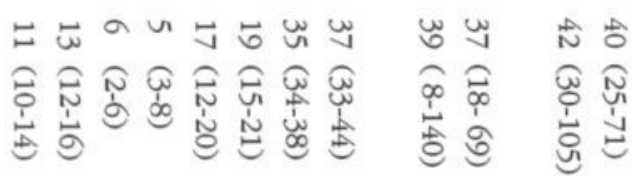
며

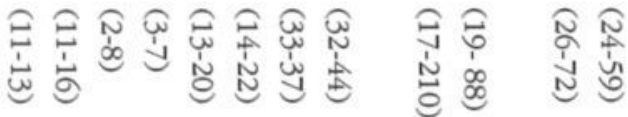

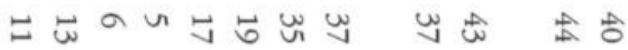

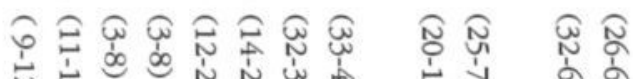

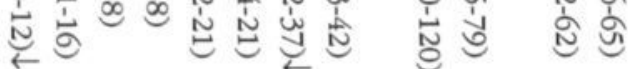

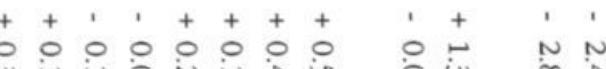

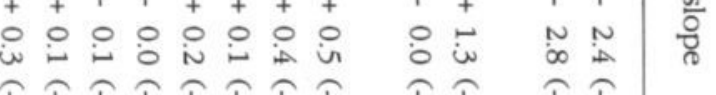
ด 0 ก w

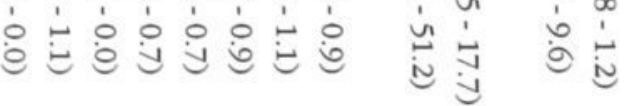




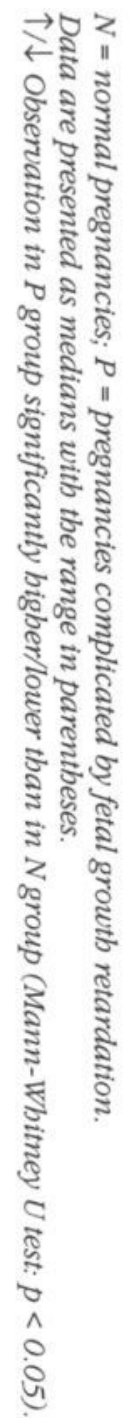

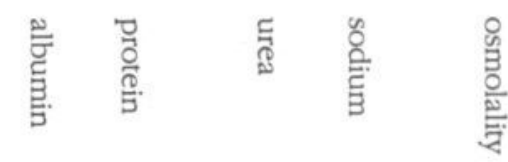

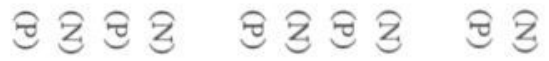

जิ

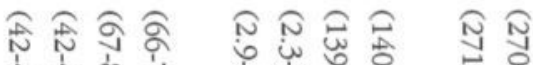

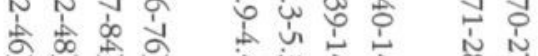

点造递

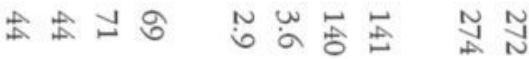

在全金

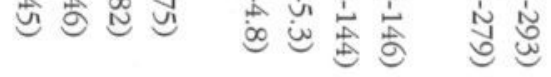

屯ै

蛋

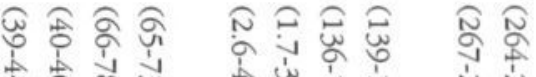

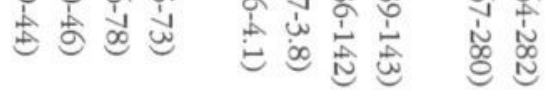

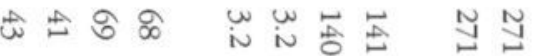

$\infty$

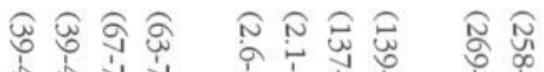

在古 额

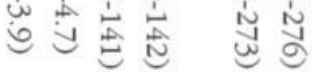

屯ै

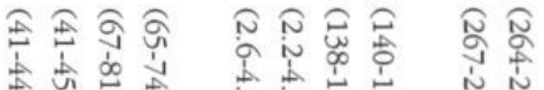

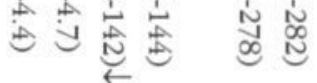

는

4

공

$\$$



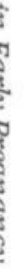




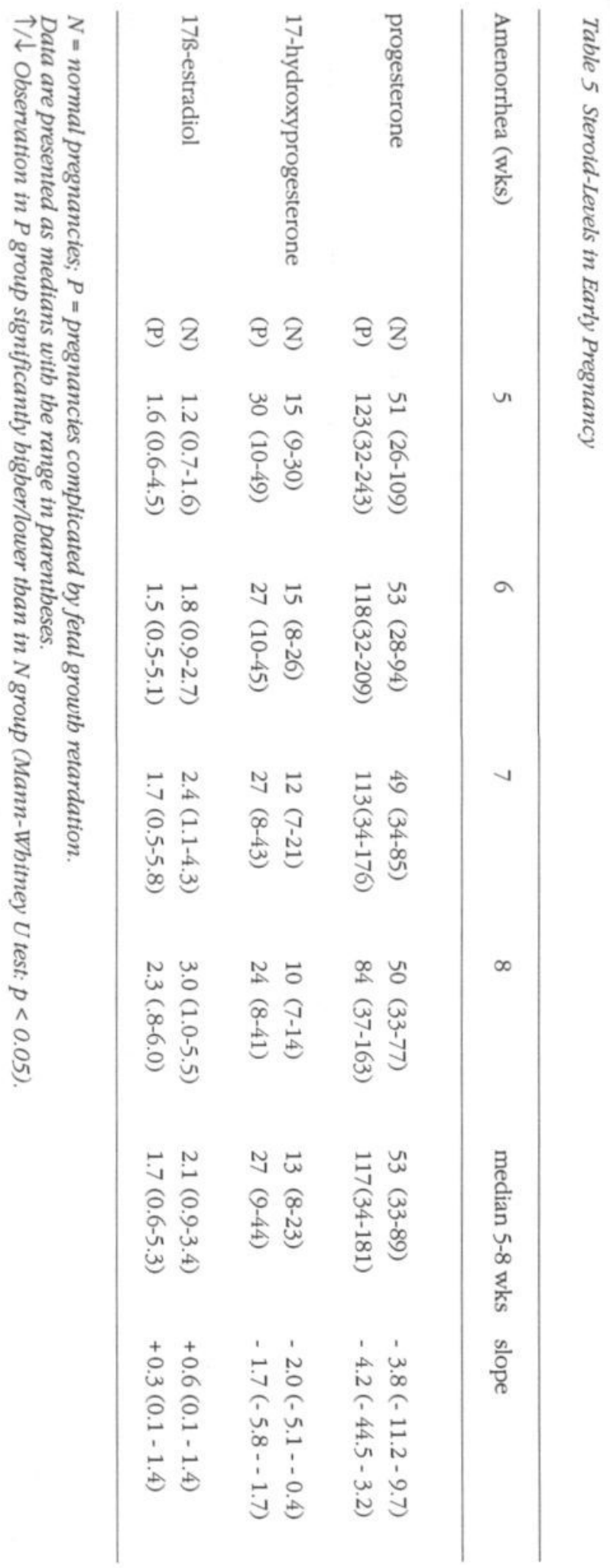



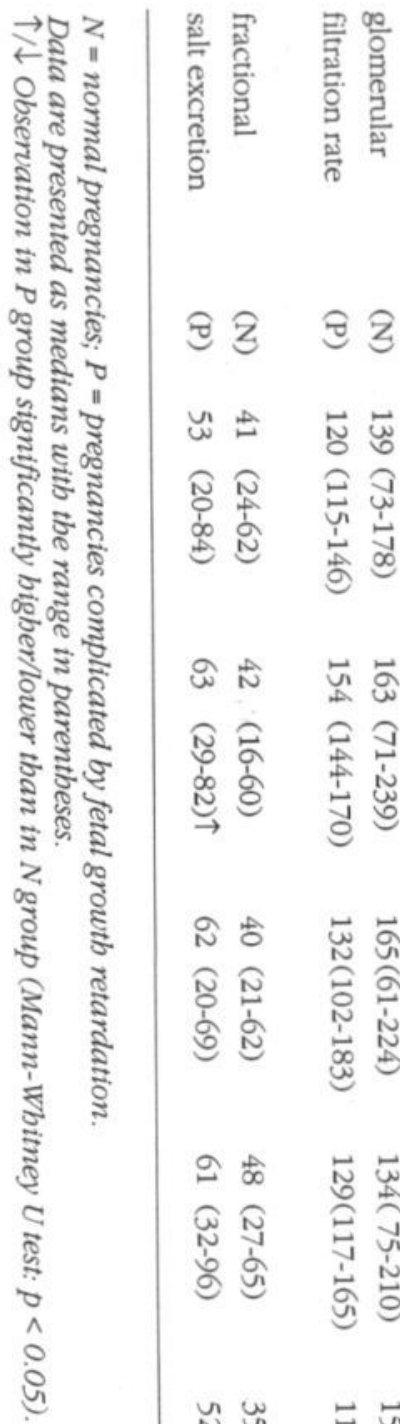

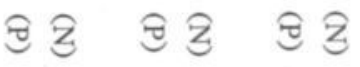

嵌ث

กิ సิ

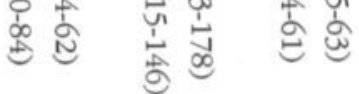

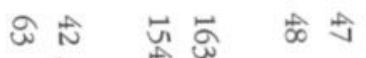

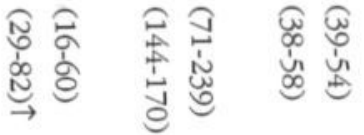

สี้

กิ

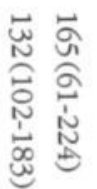

के से

5

กิ సิ

웡

है ज्ञ

9

స్

合

స్ సิ

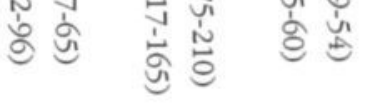

फ़

ज顸

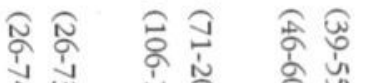

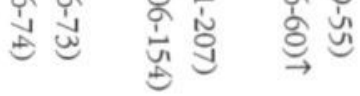

N

w

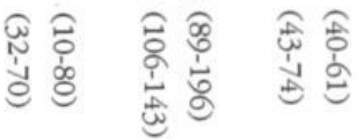

ఉึ

NN N

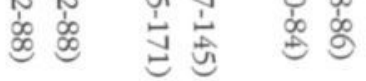




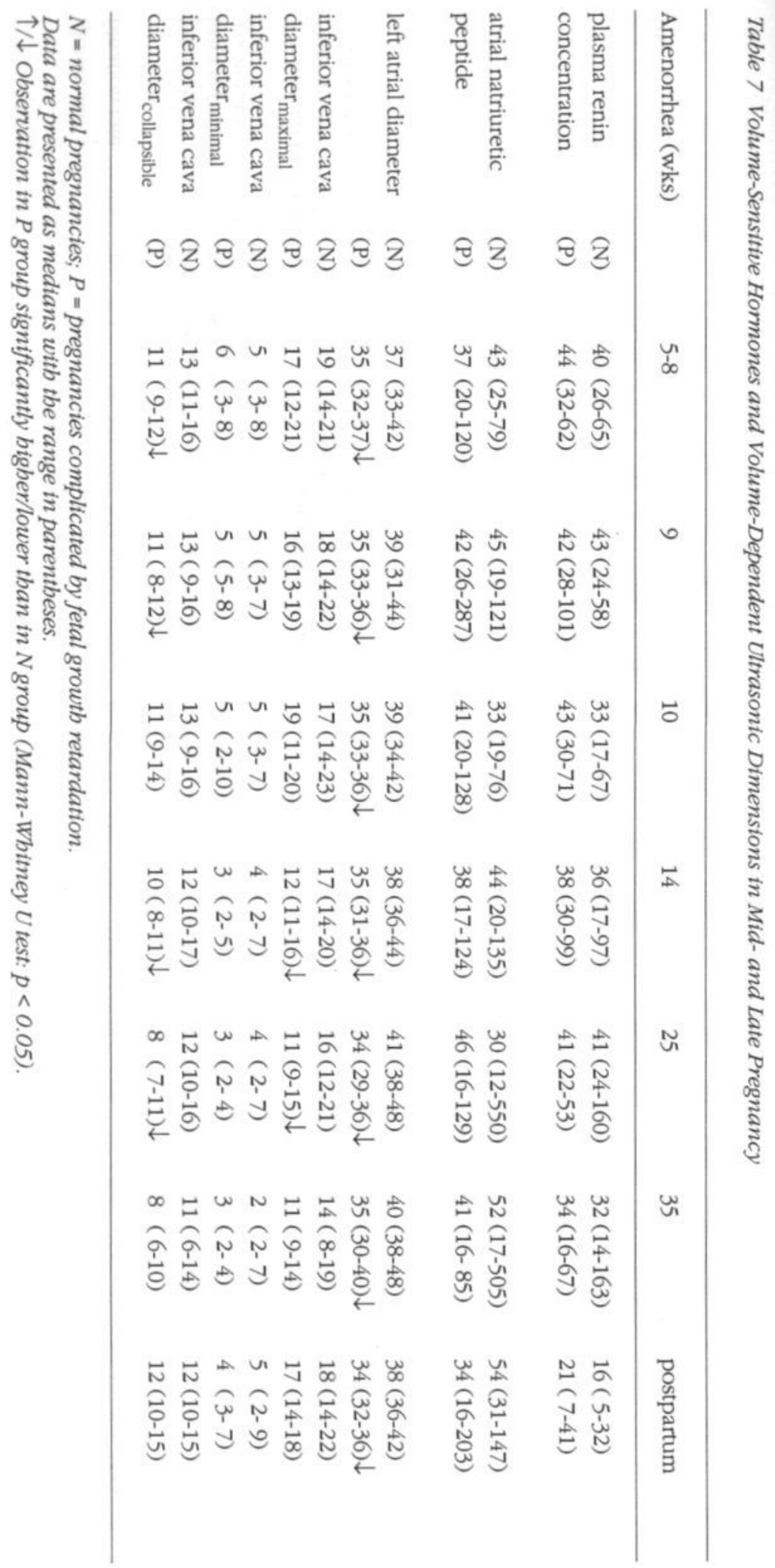




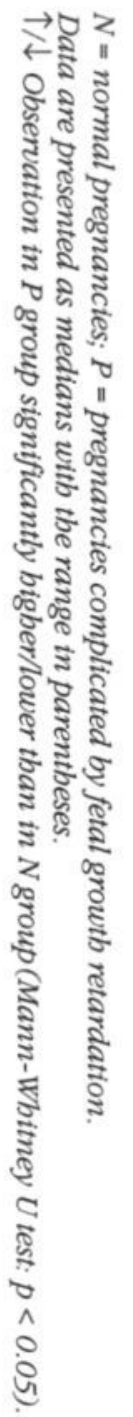

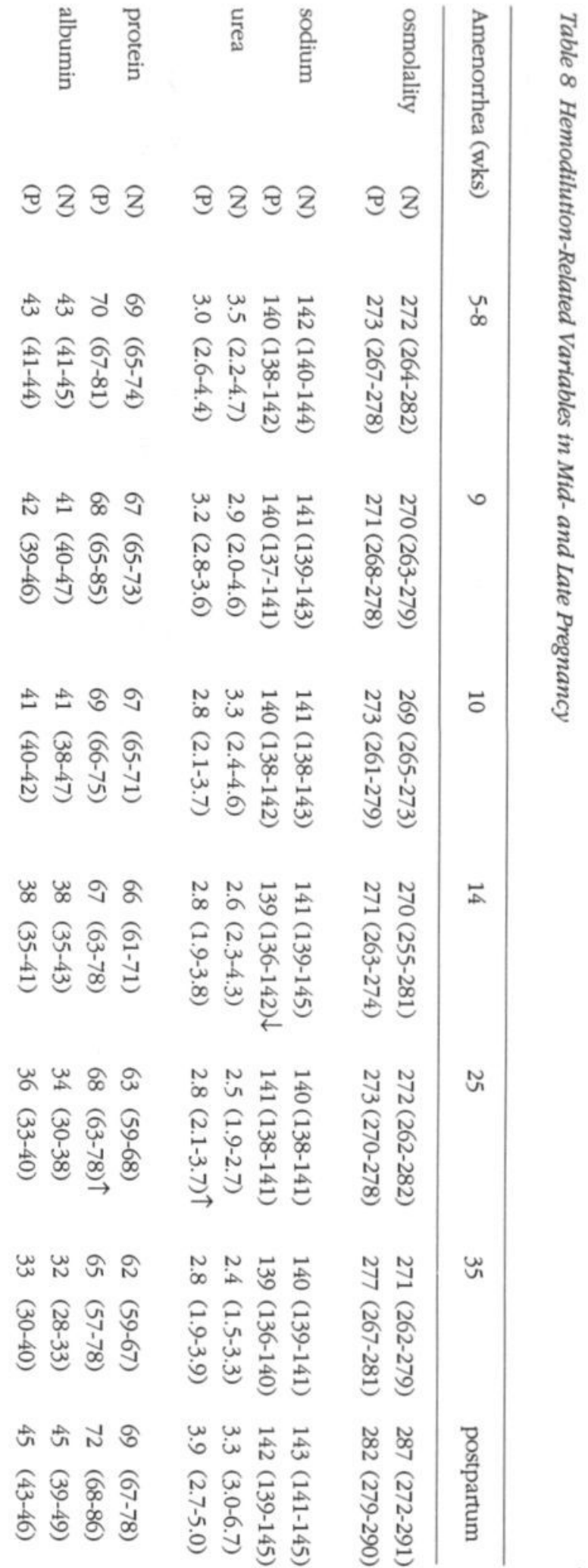




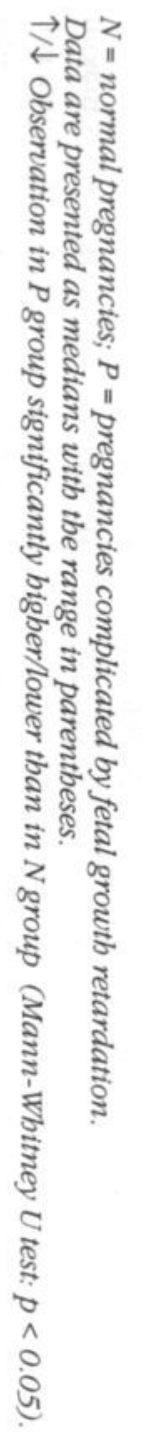

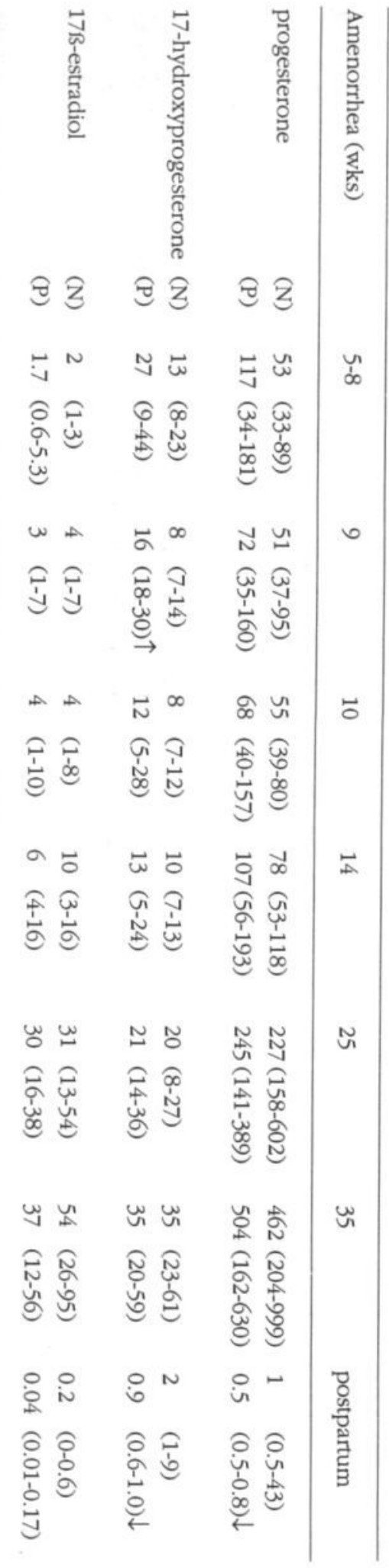

है 


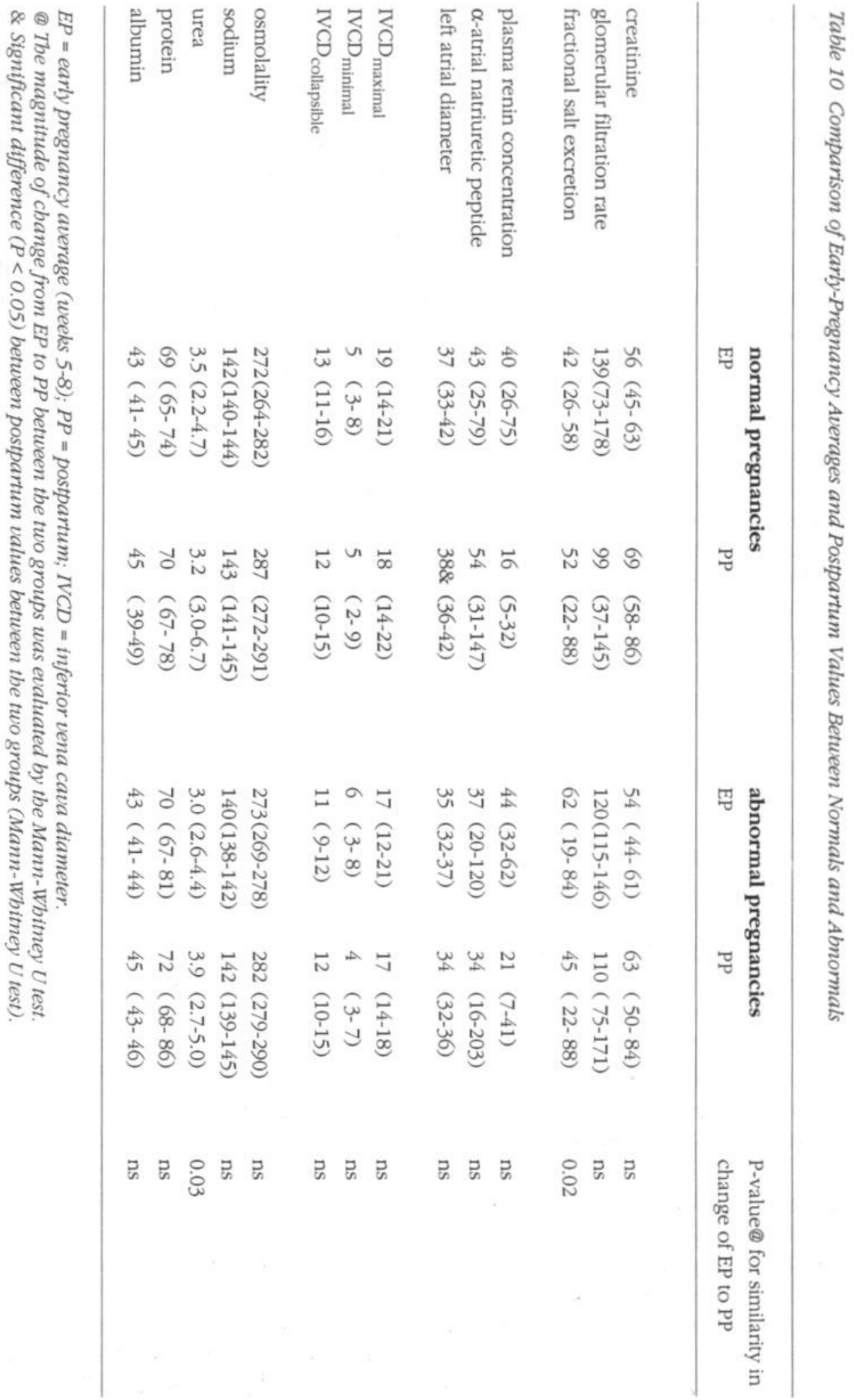




\subsection{Discussion}

In a previous study describing the maternal hemodynamic adaptation to pregnancy we found evidence for a transient fall in vascular filling state in the first weeks of pregnancy (Duvekot et al., 1993a). This observation led us to follow longitudinally volume-related variables during the first weeks in pregnancies subsequently complicated by fetal growth retardation. The aim of the study was to determine whether these pregnancies are preceded by a comparable transient fall in vascular filling or not. This information was expected to increase the insight in the pathogenesis of the hypovolemia that accompanies manifest fetal growth retardation (Goodlin et al., 1981).

In our study, pregnancy loss was 8 of 24 . This high incidence was explained, in part as related to the early diagnosis of pregnancy in these women ( 5 women aborted before the sixth pregnancy week), and in part as being inherent to the poor pregnancy performance in a subfertile population (Coulam 1992). The latter was also considered to have contributed to the high incidence of fetal growth retardation (6 of 24) in our subjects (Williams et al., 1991). Nevertheless, an uncomplicated course of pregnancy resulting in the birth of a healthy, appropriately grown infant, assured in our view a normal maternal adaptation, irrespective of the former cause of the subfertility. By choosing nonparametric tests and evaluating changes in a wide variety of complementary variables it was possible to discern differences between normal pregnancies and pregnancies complicated by fetal growth retardation, in spite of the modest group sizes. To our knowledge, the maternal volume adaptation has never been studied prospectively and longitudinally in the first weeks of pregnancies subsequently complicated by fetal growth retardation.

Left atrial diameter was consistently smaller in the women destined to deliver growth restricted infants, not only throughout pregnancy but also postpartum. The latter observation was accompanied by a faster mean velocity of circumferential fibre shortening (Duvekot et al., 1993b), a phenomenon consistent with a higher cardiac contractility. Smaller vascular dimensions in combination with a higher cardiac contractility may point to a marginal filling state of the vascular bed triggering a compensatory rise in cardiac contractility to maintain cardiac output. As a result the circulation becomes "hyperdynamic" which implies that insufficient venous return induces a rise in the ratio of heart rate to stroke volume so as to maintain cardiac output. Whether this different postpartum hemodynamic state after pregnancies complicated by fetal growth retardation indicates incomplete recovery from complicated pregnancy or a pre-existing condition, remains to be determined. A pre-existent marginal vascular filling state may imply limited reserves to compensate from a superimposed reduction in vascular filling state, which seems to develop shortly after implantation. This speculation is supported by the clinical observation that the risk of repeat instances of fetal growth retardation is increased in women with a previous history of fetal growth retardation (Patterson et al., 1986).

In a recent study, we demonstrated that left atrial diameter varies as a function of vascular filling state (Duvekot et al., 1994a). Other studies provided evidence for the inferior vena cava collapsible $_{\text {and inferior vena cava }}$ minimal to represent indirect 
data with respect to the more pronounced vascular underfill state in the abnormal estimates for venous return (stressed volume) and basal vascular filling (unstressed volume), respectively (Rothe 1986; Duvekot et al., 1993a; Duvekot et al., 1994b). In the abnormal group the early-pregnancy mean inferior vena cava collapsible $_{\text {was, like }}$ the left atrial diameter, smaller than in the normal group. Since these observations resemble those in clinical conditions of acute hypovolemia (Rothe 1986), they support a prolonged state of reduced preload in the early stages of pregnancies complicated by fetal growth retardation. At 25 and 35 weeks, the smaller left atrial diameter was accompanied by a smaller inferior vena cava maximal $_{\text {and inferior vena cava }}$ collapsible. Apparently, the initial relative hypovolemia deteriorates with advancing pregnancy. This view is supported by direct plasma volume measurements (Hytten and Paintin, 1963; Gibson 1973; Goodlin et al., 1981).

For reasons specified before blood volume was not measured directly. Instead, we intended to show a possible difference in the early-pregnancy volume adaptation by combining the ultrasonic variables with the levels of the volume-sensitive hormones $\alpha$-atrial natriuretic peptide and plasma renin concentration. In the normal group the apparent decrease in left atrial diameter before the fifth week was indeed accompanied by a lower early-pregnancy mean $\alpha$-atrial natriuretic peptide relative to postpartum values (Table 10). A similar phenomenon has also been reported to occur in early baboon and rat pregnancy (Phippard et al., 1988; Castor et al., 1989). A more pronounced and prolonged reduction in vascular filling state in the abnormal group, as suggested by the ultrasonic observations, should be paralleled by a lower $\alpha$-atrial natriuretic peptide, and a higher and non-falling plasma renin concentration. Neither the early-pregnancy means nor their rates of change in early pregnancy differed between the two groups. However, the large variability in both hormone levels with coefficients of variation exceeding $50 \%$, together with the limited group sizes, limited the potential role of these hormones to support or oppose the ultrasonic data.

Already in the fifth week of pregnancy osmolality decreases rapidly (Davison et al., 1981b), which occurs most likely in response to nonosmotically-induced release of vasopressin (Schrier and Briner, 1991). The latter is triggered by a state of arterial underfill, a condition that probably develops in early pregnancy in response to the initial systemic vascular relaxation (Schrier and Briner, 1991; Duvekot et al., 1993a). In early pregnancy we measured a number of variables known to vary with hemodilution. In the group of pregnancies complicated by fetal growth retardation the earlypregnancy mean sodium concentration was lower and the rate of decrease in serum urea smaller than in the normal group. The lower mean serum sodium, with no trend to decrease between 5 and 8 weeks in the abnormal group, suggests that this variable reached its lowest level already by the fifth week. In contrast, in the normal group serum sodium did not reach its lowest level before the seventh week. Serum urea levels decreased at a slower rate in the abnormal group. The lack of a difference between the other hemodilution-related variables in the two groups is probably related to the larger measurement error. Nevertheless, these observations do support the idea that in the abnormal pregnancies the extra volume which can be mobilized by physiologic hemodilution (Davison et al., 1981b; Schrier and Briner, 1991) may be entirely exploited by the fifth week. Such a response not only supports the ultrasonic 
group, they also indicate that the osmoregulatory system is functioning normally in these subjects.

The expected effect on renal function of a prolonged and more pronounced early-pregnancy vascular underfill state in the abnormal group as compared to the normal group, would be a lower glomerular filtration rate with a slower rate of increase. The effect on the serum creatinine concentration would be opposite. In the present study, only the latter phenomenon was observed. The glomerular filtration rate increased in both groups. However, it was not possible to discern a difference in the rate of increase, most likely also related to the large measurement error (coefficient of variation approximately $27 \%$ ).

The fractional salt excretion in early pregnancy was higher in the abnormal group than in the normal group, when compared to the nonpregnant (postpartum) reference (Table 10). Since no information was obtained on concomitant salt intake, it is not possible to determine whether the higher early-pregnancy fractional salt excretion in the abnormal subjects indicates a higher renal salt wastage or a higher salt intake. Nevertheless, this observation indicates that abnormalities in the sodium homeostasis could play a role in the possible early-pregnancy maladapatation in volume homeostasis.

The present study provides evidence for a more pronounced vascular underfill state already during early gestation in pregnancies complicated by fetal growth retardation as compared to normal pregnancies. In addition, this initial vascular underfill state does not seem to trigger a proper compensatory volume response in these abnormal pregnancies. As a consequence, the initial, normally transient, relative hypovolemia not only persists beyond eighth weeks, it also deteriorates with advancing pregnancy. The evidence for this concept was primarily based on the echocardiographic data. Although the other volume-related variables did not contradict this concept, their support for it was limited, partly because of the relatively large variability associated with their measurement, partly because of the limited group sizes. The early-pregnancy difference in the mean left atrial diameter between the two groups was $2 \mathrm{~mm}$, a difference of at least one standard deviation. When using a two-sample t-test, 21 individuals per group would be required with a power 0.90 to detect a difference between two expected means of one standard deviation (Pocock 1983). It follows that the results of this study require confirmation in a larger population.

\subsection{Acknowledgements}

This study was supported by a grant from Schering Nederland BV, Weesp, The Netherlands. The authors wish to acknowledge Albert F. Grootendorst MD, $\mathrm{PhD}$ and Hubert J.A. Schouten PhD, for their statistical assistance. 
Chapter 9

GENERAL DISCUSSION 
Pregnancy is characterized by early maternal changes in circulatory hemodynamics. Until recently, changes in the three constituents of this adaptation, cardiovascular hemodynamics, renal hemodynamics and volume homeostasis, had only been partially correlated. The importance of obtaining a better understanding of this adaptation may be twofold. Firstly, the risk of pregnant subjects with preexisting cardiovascular disease developing a superimposed preeclampsia may become more predictable and, with it, possibly more accessible to preventive measures. Secondly, disclosure of the initial adaptation may provide the tools for developing, for instance, a test in the menstrual cycle for estimating the adaptive reserve capacity in subsequent pregnancy. Such a test could be used to determine the risk profile of a woman with regard to fetal growth retardation and preeclampsia. In late pregnancy these complications are characterized by alterations in the cardiovascular system and volume homeostasis. Both diseases still account for a large fetal and maternal mortality in the Western world (Högberg et al., 1994).

In an effort to unravel the mechanisms of the maternal circulatory adaptation to pregnancy, we performed an observational study in young and healthy pregnant women. The first weeks of gestation were chosen as the most appropriate period to study this adaptation. In this period of pregnancy the changes in cardiovascular dynamics, renal function and possibly related steroid environment are most profound. The recruitment of the subjects from a subfertile population yielded the following advantages. The time of conception was exactly known on the basis of ultrasonic ovulation detection, thus providing an exact timing of gestational age. All subjects were familiar with clinical assessments by ultrasound, blood sampling and regular visits to the hospital, which had a positive effect on the subjects' motivation to participate in the study and reduced the strain of the measurements, which was of the utmost importance during the echocardiographic studies. Another anticipated advantage was the large proportion of pathological pregnancies. The possible bias introduced by the selection of subjects from this population was corrected for by redefining normality: a normal and uncomplicated course of pregnancy resulting in the birth of a healthy appropriately grown infant in our view assured a normal maternal adaptation, irrespective of the initial cause of the subfertility. For medical-ethical reasons, none of the measurement procedures employed in this study were invasive. The advantage of this approach is a minimal risk to the mother and her conceptus, and the possibility to perform repeated measurements in this particularly vulnerable period of pregnancy. On the other hand, noninvasive procedures are often indirect, which limits their strength, while some of the procedures used in this study, such as creatinine clearance and the ultrasonic measurement of small vascular dimensions, are also hampered by relatively large measurement errors. Nevertheless, repeated measurement of a wide spectrum of physiologically related variables at least partly neutralized the impact of these disadvantages.

Non-invasive ultrasound techniques offer the opportunity to perform safe, longitudinal echocardiographic studies in the earliest stages of pregnancy. In recent years, measurement of cardiac performance by M-mode echocardiography, both with and without simultaneous Doppler measurements, has been validated in pregnancy against standard invasive methods. Since pregnancy may be regarded as a state of 
chronic vascular underfill or overfill (Schrier and Dürr, 1987), we were also interested in the degree of vascular filling. The first part of this study tested the hypothesis whether the echocardiographic parameters routinely in use are valuable estimates of baseline blood volume, vascular filling state and changes in these parameters. Since a large proportion of the blood volume is accommodated in the large central veins, serving as a blood volume reserve, the inferior vena cava dimensions before and during deep inspiration were tested as new and non-invasive parameters for blood volume and vascular filling state, both in male and female subjects. Neither the normal echocardiographic parameters nor the inferior vena cava dimensions correlated with baseline blood volume. Nevertheless, this study provided evidence that blood volume and ultrasound dimensions differ between the sexes. This implies that validation of possible relationships should be performed separately in each gender. The experimental procedure used to impose a rise in vascular filling state proved to be an appropriate model for increments in preload. The rapid infusion rate provoked neither a reduction in blood pressure nor a rise in cardiac output. The routinely determined echocardiographic parameters left atrial diameter and left ventricular end-diastolic dimension proved to be reliable qualitative parameters for detecting changes in vascular filling state. One of the most important findings with respect to the clinical studies in the second part of the present study was that the baseline echocardiographic dimensions had no predictive value whatsoever with regard to the response to volume loading. The effect on the inferior vena cava dimensions of changes in vascular filling were also followed during volume expansion. The change in ultrasonic inferior vena cava indices during volume loading was largest for the minimal and maximal inferior vena cava diameter. The shape of the caval contour had not changed during the volume loading and the vena cava diameter was found to have increased as a function of the infused fluid volume. Although we did not perform volume depletion experiments, the linear relationship is likely to hold also in a condition of vascular underfill. This assumption is based on in vitro observations with respect to a volume response curve of the inferior vena cava diameter which was symmetrical and S-shaped (Moreno et al., 1973). From the response of the inferior vena cava dimensions during deep inspiration and volume loading it was concluded that the minimal inferior vena cava diameter provides an indirect estimate of unstressed volume, while the collapsible part of the inferior vena cava estimates stressed volume. Although in clinical practice this finding seems of little practical value, these estimates may provide useful information in the study of the (patho)physiology of volume regulation, e.g. in pregnancy. Changes in venous compliance are likely to be translated into changes in venous diameter, indicating that the latter is to vary as a function of venous capacity. This implies that the width of this larger blood vessel under physiological conditions is consistent with changes in vascular compliance. The results of our experiments were compatible with this concept. The expected increase in compliance as a result of stress-relaxation was accompanied by an increase in caval dimensions.

The results with respect to the maternal circulatory adaptation to early pregnancy have been elaborated in the second part of this thesis. Ten pregnancies proceded uneventful and the data from these subjects were analyzed. For practical reasons, no prepregnancy reference values had been obtained. Although for most variables tes- 
ted, three months postpartum data could be expected to have returned to their prepregnancy levels, the 5 weeks value was chosen to serve as a reference for the earlypregnancy data points. Most data displayed the largest changes in the $5-8$ weeks period. Therefore, this period was chosen for more thorough statistical evaluation. The limited sample size obstructed statistical confirmation of normal data distribution. For this reason, the data were analyzed by nonparametric tests. Since most parameters are likely to have begun to change shortly after implantation, only the final portion of the early changes will be concentrated in the $5-8$ weeks period. To maximize the strength of the analysis, the data were examined by trend detection, consisting of applying the Wilcoxon signed rank test to the individual linear regression lines. This statistical approach allowed the recognition of a consistent pattern in the measured parameters over this three weeks period, despite the small magnitude of most changes. Obviously, all women were in good health during their early pregnancy and both the data points and the absolute changes observed in the present study were within physiological ranges.

The hypothesis is postulated that in early pregnancy an as yet unidentified stimulus induces an overall decrease in vascular tone, leading to systemic vasodilatation and a rise in arterial compliance, both factors independently contributing to a fall in afterload. Stroke volume increases in response to this afterload reduction. Presumably, the reduced tone of the vascular wall, together with the vasodilatation, is directly responsible for both the downregulation of the baroreceptor and the nonosmotically induced release of vasopressin (Schrier and Briner, 1991). The latter leads to hemodilution and hence to a reduction in blood viscosity. These changes together are responsible for the establishment of a high flow/low resistance circulation in early pregnancy. The rise in glomerular filtration rate in concert with the rise in cardiac output is considered to result from the relaxation of afferent and efferent renal vessels. The increase in left atrial diameter and the fall in plasma active renin concentration between the fifth and eighth pregnancy weeks and the plateauing of the glomerular filtration rate after the eighth week support a rise in vascular filling state, probably to compensate for an initial state of hypovolemia. The lack of changes in the inferior vena cava dimensions may indicate that the postulated transient mismatch between size and filling state of the vascular bed develops predominantly in arteries because of their higher smooth muscle content. This may explain why we were unable to detect changes in the inferior vena cava diameter.

Several mechanisms have been proposed about the nature of this potent, pregnancy-related, vasodilator. Some of the prostaglandins have strong vasodilatory effects (Broughton Pipkin et al., 1987), but are unlikely candidates for involvement in this early-pregnancy vasodilation, as suggested by the observation that cyclooxygenase inhibitors are unable to neutralize the rise in glomerular filtration rate in rats during midpregnancy (Conrad and Colpoys, 1986). The pregnancy hormones, such as estrogen, progesterone, prolactin, hCG and hPL, were unable, alone or in combination to reproduce the early-pregnancy fall in vascular tone in animal studies (Barron 1987). A promising second messenger in establishing the vascular effect is endothelial-derived relaxing factor, of which nitric oxide is one form (Katz 1988). This molecule exerts a strong vasodilatory effect on the resistance vessels. 
Endothelial-derived relaxing factor acts as an endogenous nitrovasodilatator. Its release is stimulated by neuropeptides and agents released during platelet activation and thrombosis. The nitric oxide release accelerates, for instance, in response to calcitonin gene-related peptide, which is distributed in the perivascular nerves. Plasma calcitonin gene-related peptide is increased throughout pregnancy (Stevenson et al., 1986). In pregnant rats endogenous nitric oxide enhances the vasodilatation by modulating vascular responses to sympathetic nerve activity and circulating catecholamines (Beilin and Chu, 1993). Inhibition of endothelium-derived relaxing factor by free hemoglobin has been hypothesized to be involved in the pathogenesis of preeclampsia (Sarrel et al., 1990). The study of possible links between the paracrine and autocrine interactions at the feto-maternal interface and the enhanced release of factors that raise endothelium-derived relaxing factor forms the next logical step in the unraveling of the presumed early-pregnancy generalized vasodilatation. In this context cytokines with their ability to sensitize the endothelial cells, have recently been mentioned as possible links in the chain of events leading to generalized vascular relaxation (Fay and Grudzinskas, 1991; Daunter 1992; Vinatier et al., 1992).

Using the above concept, the data obtained in pregnancies complicated by intrauterine growth retardation (IUGR) were compared with data from normal pregnancies. Two of the IUGR pregnancies were also complicated by transient hypertension. Although the pathophysiological mechanisms leading to fetal growth retardation and preeclampsia might be different, we did not exclude these pregnancies from our study. This decision was based on the finding that pregnancies complicated by IUGR and by IUGR and preeclampsia both show decreased plasma volumes in late pregnancy as compared to normal and preeclamptic pregnancies, respectively (Nisell et al., 1988; Arias 1975; Hays et al., 1985; Sibai et al., 1983). Both conditions seem to share an identical early circulatory maladaptation to pregnancy. Because of the modest number of subjects in each group we chose to use the Mann-Whitney U test to compare the variables determined in the "abnormal" group complicated by fetal growth retardation with those in the normal group. For each of the ten normal and six abnormal pregnancies, (linear) regression lines were computed for the relationships between the measured variables (dependent) and gestational age between five and eight weeks (independent). Furthermore, the early-pregnancy means for this period were calculated for each individual, and then compared between the two groups. By this approach the four intra-individual data points for each variable were summarized as recommended for the analysis of serial measurements between two groups (Matthews et al., 1990). Comparison of cardiovascular data showed that in early pregnancy the abnormal group differed from the reference group by a failure to increase cardiac output and by a consistently smaller and non-increasing left atrial diameter. These findings are indicative of a state of inadequate vascular filling even in the first weeks of pregnancy, persisting after the eighth week. Although the other volume-related variables pointed also in the direction of this concept, their support for it was limited, partly because of the relatively large variability associated with their measurement, partly because of the limited group sizes. In IUGR pregnancies the initially induced underfill state failed to become adequately compensated, despite apparently normal activation of volume retaining mechanisms. Possible expla- 
nations for this early circulatory maladaption in pathological pregnancies could be an insufficient or excessive initial vasorelaxant/vasodilatating stimulus, or alternatively, an inadequacy of the volume-retaining mechanisms to compensate for the vascular underfilling. The presence of lower levels of hCG in the first trimester of pregnancies subsequently complicated by fetal growth retardation could be an indication for the first mechanism (Johnson et al., 1993). However, this possibility is contradicted by the apparent normal activation of the volume homeostatic mechanisms (Chapter 8). Evidence for inadequate volume conservation is provided by the patterns of change in the inferior vena cava dimensions in the abnormal group. The early-pregnancy reduction of the minimal inferior vena cava diameter suggests the mobilization of unstressed volume, which usually does not occur unless the vascular bed is (relatively) depleted (Rothe 1986). It is tempting to speculate that a primary inability to rebalance the vascular filling interferes with trophoblast development. This mismatch may raise sympathetic tone, which in turn, has an adverse effect on the growing environment of the trophoblast. Since the exact sequence of events is still obscure, further studies are needed to elucidate the underlying pathogenesis of maladaptation.

Although the percentage of abnormal pregnancies in the initial population had been expected to be elevated, it turned out to be surprisingly high. The high incidence of fetal growth retardation $(6 / 16)$ in our subjects is significantly higher than $10 \%$ (95\% confidence intervals: $15-65 \%$ ). This finding may be related to the poor reproductive performance of subfertile subjects (Williams et al., 1991). The number of spontaneous abortions was also very high (8/24). This high incidence may be explained threefold. Because of the early recognition of pregnancy, early pregnancy losses were also defined as spontaneous abortions. Five women aborted before the sixth week of pregnancy. At least one of these early abortions could be defined as subclinical pregnancy loss (Bateman et al., 1992). Secondly, our infertility clinic is a tertiairy referral center with possibly an even more unfavorable population with respect to pregnancy performance. Several investigators have shown a higher incidence of spontaneous abortion among infertile couples (Coulam 1992). In the third place, women conceiving during hormonally manipulated cycles are prone to poor reproductive performance, probably based on maladaptation of the endometrium (Forman et al., 1989; Rogers et al., 1991). Disturbances in the steroid environment during ovulation leading to unfavorable conditions at the implantation, are possibly responsible for this phenomenon (Forman et al., 1988). In our study steroid hormone levels did not differ between normal and abnormal pregnancies during early pregnancy. Women that conceived after treatment with gonadotrophins were not included in our study.

The strength of the statistical analysis of our data was limited by the small numbers of normal and pathological pregnancies. Therefore, the results should be interpreted cautiously. On the other hand, the difficulties encountered in collecting this type of data prospectively, longitudinally and in early pregnancy, and in subjects eventually developing fetal growth retardation, are large, which increases their value enormously. Other studies evaluating maternal adaptation to pregnancy longitudinally have always described small numbers of subjects, comparable to that in the pre- 
sent study (Davison and Noble, 1981a; Davison et al., 1981b; Robson et al., 1989a). It follows that firm conclusions on the pathophysiologic mechanism of fetal growth retardation require a study design with a very explicit hypothesis, preferably to be tested in a larger number of subjects.

Finally, the present study was designed to investigate if, why and how certain women who develop fetal growth retardation in advanced pregnancy differ from a control group not only in early pregnancy but also in the nonpregnant state with respect to cardiovascular parameters. It was clearly shown that subjects in the abnormal group, in the absence of demographic differences, had a hyperdynamic circulation on the basis of a smaller left atrial diameter and larger cardiac contractility. The use of postpartum data for this comparison, however, limits its strength. Prepregnant values are necessary if we wish to confirm the suggestions made in the present study. This is especially true since volume homeostasis is likely to remain altered for a long period after delivery (Finn et al., 1991).

Hytten's 1976 statement (Hytten 1976) about progress in understanding of maternal adaptation is equally applicable today, and to the study of early maternal circulational adaptation: "The ability to produce a large increase in plasma volume is one of the hallmarks of a successful pregnancy ". 
Chapter 10

SUMMARY 
Maternal adaptation to human pregnancy consists of profound changes in hemodynamics, renal function and volume homeostasis. Most hemodynamic and renal adjustments are already fully expressed by the end of the first trimester, with the exception of plasma volume which increases gradually from early pregnancy until the beginning of the third trimester. Most of our current knowledge about these adjustments has been extrapolated from the difference between the midpregnant and nonpregnant states. Consequently, both the underlying mechanism and the functional meaning of the early-pregnancy changes are poorly understood.

In pregnancies complicated by fetal growth retardation or preeclampsia, the normal rise in cardiac output, glomerular filtration rate and plasma volume is either reduced or absent. Already in midpregnancy, these subjects differ from subjects with uneventful pregnancies by defective plasma volume expansion and cardiac output rise. It is still unsettled whether these midtrimester abnormalities are primary or reflect ongoing maladaptation from very early pregnancy on. The first and second chapter of this thesis consist of a review of the current literature on the adaptations in hemodynamics and volume homeostasis in normal and in complicated pregnancy.

Non-invasive ultrasound techniques offer the opportunity to perform safe, longitudinal echocardiographic studies in the earliest stages of pregnancy. Since pregnancy may be regarded as a state of chronic vascular underfill or overfill, we were also interested in the degree of vascular filling. In the first part of this study we tested whether useful information on baseline blood volume, vascular filling state and a change in these parameters can be obtained by echocardiography. Since a large fraction of the blood volume is accommodated in the large central veins, the inferior vena cava dimensions before and during deep inspiration were tested as new and non-invasive parameters for blood volume and vascular filling state, both in male and female subjects. With respect to the subsequent clinical studies this validation study revealed that the routinely-measured echocardiographic parameters and the inferior vena cava dimensions were unreliable in estimating baseline blood volume and filling state. Nevertheless, some ultrasonic dimensions can provide reproducible qualitative information on acute increments in vascular filling state. In response to a rapid volume loading, the routinely measured echocardiographic parameters, left atrial diameter, right atrial area, and left ventricular end-diastolic dimension described changes in vascular filling state best. The diameter of the inferior vena cava increased in proportion to the infused amount of plasma expander without appreciable change in the caval contour. From the changes in the inferior vena cava dimensions in response to volume loading, it was concluded that the minimal diameter and collapsible part of the inferior vena cava provide indirect estimates of unstressed and stressed volume, respectively.

The results with respect to the maternal hemodynamic adaptation to early pregnancy have been elaborated in the second part of this thesis. To participate in this study, healthy, normotensive women were recruited in consecutive order from our subfertility clinic as soon as pregnancy was established. Several variables related to hemodynamics and volume homeostasis were measured at weekly intervals between the fifth and tenth weeks of pregnancy and 3 months postpartum. The pregnancies of 10 subjects evolved uneventfully and the data from these subjects were analyzed. 
Although most variables tested are likely to have returned to their prepregnancy levels by 3 months postpartum, we used the 5 weeks' value as a reference for the early-pregnancy data points. The largest change in most variables took place in the period between 5 and 8 weeks of pregnancy. Therefore, this period was chosen for more thorough statistical analysis. Because of the limited sample size we decided to analyse the data using nonparametric statistics. For each subject the trend of change of each variable was summarized by calculating the regression line through the 4 individual data points between 5 and 8 weeks. By this statistical approach it was possible to discern consistent early-pregnancy patterns in the measured variables, despite the small magnitude of most changes. On the basis of the results in these 10 pregnant individuals, the hypothesis was postulated that in early pregnancy an as yet unidentified stimulus induces an overall decrease in vascular tone causing systemic vasodilatation and a rise in vascular compliance. The latter two effects lead to a fall in cardiac afterload and with it, a compensatory rise in cardiac output. These effects together with the higher systemic flow rate, are expected to contribute to the downregulation of the baroreceptor and the nonosmotically induced release of vasopres$\sin$. The latter leads to hemodilution. This set of changes enables the establishment of a high flow/low resistance circulation in early pregnancy, with a chronically elevated cardiac output that does not require extra cardiac work (cardiac output times mean arterial pressure). The rise in glomerular filtration rate in concert with the rise in cardiac output is considered to result from the relaxation of afferent and efferent renal vessels. The rise in left atrial diameter and the fall in plasma active renin concentration between the fifth and eighth pregnancy weeks and the plateauing of the glomerular filtration rate after the eighth week support a rise in vascular filling state, probably to compensate for an initial state of (relative) hypovolemia as a result of the gegeneralized vasorelaxation. The lack of changes in the inferior vena cava dimensions may indicate that the postulated transient mismatch between size and filling state of the vascular bed develops predominantly in arteries because of their higher smooth muscle content. As a consequence, changes in the inferior vena cava may have been too small to be detectable by this technique.

Using the above concept, the data obtained in six pregnancies complicated by fetal growth retardation were compared with data from normal pregnancies. Comparison of cardiovascular data showed that in early pregnancy this abnormal group differed from the reference group by a failure to increase cardiac output and by a consistently smaller and non-increasing left atrial diameter. Although the results of the other volume-related variables did not contradict this indication of a diminished volume expansion, their support for it was limited. This was partly because of the relatively large variability associated with their measurement, partly because of the limited group size. These findings are indicative of a state of inadequate vascular filling even in the first weeks of pregnancy, persisting after the eighth week. In pregnancies complicated by fetal growth retardation the initially induced underfill state failed to become adequately compensated, despite normal activation of volume retaining mechanisms. However, since normal activation of the volume homeostatic mechanisms was demonstrated to occur in this study, the quality of the initial stimulus seems adequate. Evidence for inadequate volume conservation is provided by the 
pattern of change in the inferior vena cava dimensions in the abnormal group. The early-pregnancy decrease in the minimal inferior vena cava diameter suggests the mobilization of unstressed volume, which usually does not occur unless the vascular bed is (relatively) depleted.

Finally, in the present study we also evaluated postpartum to what extent the nonpregnant state of the vascular bed in women who gave birth to a growth retarded infant, differed from that in women who delivered of an appropriately grown newborn. It was noted that subjects in the former group, in the absence of demographic differences, had a hyperdynamic circulation as indicated by a smaller left atrial diameter and larger cardiac contractility. The use of postpartum data for this comparison, however, limits its strength.

In conclusion, the results of the studies in this thesis indicate that:

1. maternal hemodynamic adaptation to pregnancy is already detectable by the 5 th week of pregnancy,

2. the first systemic change in maternal hemodynamics appears to be a generalized vascular relaxation,

3. pregnancies complicated by fetal growth retardation are preceded by abnormal hemodynamic adaptation in early pregnancy. 
Chapter 11

\section{SAMENVATTING}


De moederlijke aanpassingen aan de zwangerschap bestaan uit grote veranderingen in cardiovasculaire hemodynamiek, nierfunctie en vochthuishouding. De meeste hemodynamische en renale aanpassingen zijn reeds volledig aanwezig tegen het einde van het eerste trimester. Een uitzondering hierop vormt de toename in plasma volume, dat geleidelijk aan toeneemt vanaf de vroege zwangerschap tot aan het begin van het derde trimester. Het meeste van onze huidige kennis over deze aanpassingen is verkregen door extrapolatie van de verschillen tussen de situatie in het tweede trimester en de niet-zwangere toestand. Hierdoor is zowel het exacte mechanisme als de functionele bedoeling van deze veranderingen in de eerste weken van de zwangerschap nog vrijwel onbekend.

In geval van zwangerschappen, die gecompliceerd worden door foetale groeivertraging of preeclampsie, is de toename in hartminuutvolume, glomerulaire filtratie snelheid en plasma volume ofwel verminderd of afwezig. In het tweede trimester, ruim voor het manifest worden van klinische symptomen, is er al sprake van een verminderde toename in deze indices. Het is nog niet duidelijk of deze vroege afwijkingen primair zijn of het gevolg zijn van een proces beginnend in de eerste weken van de zwangerschap. De eerste hoofdstukken van dit proefschrift geven een overzicht van de bestaande literatuur betrekking hebbend op de adaptatie van hemodynamiek en volume homeostase in de normale en gecompliceerde zwangerschap.

Niet-invasieve echografische technieken bieden de mogelijkheid om veilige, longitudinale, hemodynamische studies te verrichten in de eerste weken van de zwangerschap. In het eerste deel van deze studie werd getest of bruikbare informatie kan worden verkregen middels echocardiografie over het basaal bloedvolume, de vullingstoestand van het vaatbed en eventuele veranderingen in deze parameters. Omdat een groot deel van het circulerend volume zich in de grote veneuze bloedvaten bevindt, werd de diameter van de vena cava inferior, voor en na diepe inspiratie, getest als nieuwe en niet-invasieve parameter voor bloedvolume en vullingstoestand van het vaatbed. De metingen werden verricht bij mannelijke en vrouwelijke (niet zwangere) vrijwilligers. Noch de gebruikelijke echocardiografische parameters, noch de dimensies van de vena cava inferior bleken te correleren met het basale bloedvolume en de mate van vulling van het vaatbed. Niettemin bleken enkele echografische dimensies wel een betrouwbare, reproduceerbare en qualitatieve maat om acute veranderingen in de vullingstoestand van het vaatbed te detecteren. Na snelle volume expansie voldeden de routine echocardiografische parameters, linker atrium diameter, rechter atrium oppervlakte en eind-diastolische diameter van de linker ventrikel het best om deze veranderingen weer te geven. De diameter van de vena cava inferior nam gelijkmatig toe met de hoeveelheid toegediend plasmavervangingsmiddel zonder opmerkelijke verandering van de contour van het bloedvat. Op basis van de veranderingen van de dimensies van de vena cava inferior in relatie tot de volume belasting, kon worden geconcludeerd dat de minimale diameter en het gecollabeerde deel van de vena cava inferior kunnen worden beschouwd als respectievelijk een indirecte maat voor het statische en dynamische deel van het circulerend bloedvolume.

De resultaten met betrekking tot de maternale hemodynamische adaptatie aan de jonge zwangerschap worden in het tweede deel van het proefschrift beschreven. 
Geselecteerd voor de studie werden gezonde, normotensieve, zwangere vrouwen, die oorspronkelijk de infertiliteits polikliniek bezochten. Wekelijks van de 5 e tot de 10e zwangerschapsweek, en 3 maanden postpartum werden naast de echocardiografische parameters verschillende variabelen gemeten, die gerelateerd zijn aan veranderingen in hemodynamiek en volume homeostase. Bij de 24 oorspronkelijk geselecteerde proefpersonen verliepen 10 zwangerschappen ongestoord en de gegevens verkregen bij deze vrouwen werden gebruikt voor nadere analyse. Ofschoon de meeste variabelen die werden gemeten postpartum vergelijkbaar zijn met antepartum waarden, werd de waarde bij 5 weken amenorroe gebruikt als referentie punt. De grootste veranderingen bleken plaats te vinden in de periode tussen 5 en 8 weken amenorroe. Derhalve werd deze periode gekozen voor het verrichten van nadere statistische analyse. Vanwege de kleine omvang van de steekproef besloten we de data te evalueren middels niet-parametrische toetsen. Voor iedere proefpersoon werd de trend van de verandering in elke variabele samengevat door de regressielijn door de 4 individuele meetpunten tussen 5 en 8 weken amenorroe. Door deze statistische benadering was het mogelijk consistente patronen te onderscheiden in de gemeten variabelen tijdens de vroege zwangerschap, ondanks vaak kleine veranderingen. Op basis van de resultaten bij deze 10 zwangere vrouwen, werd de hypothese gepostuleerd dat in de vroege zwangerschap een tot nu toe onbekende stimulus een gegeneraliseerde afname in de vaattonus teweeg brengt. Dit heeft een systemische vasodilatatie en een toename in vasculaire compliantie tot gevolg. Deze laatste twee effecten leiden tot een afname in cardiale afterload hetgeen weer een compensatoire toename in hartminuutvolume tot gevolg heeft. Samen met de hogere systemische doorbloeding dragen deze effecten bij tot een down-regulering van de baroreceptor en de niet-osmotisch gereguleerde afgifte van vasopressine. Het laatste leidt tot hemodilutie. Deze cascade van veranderingen leidt in de vroege zwangerschap tot een circulatie met hoge bloedstroomsnelheden bij lage vaatweerstand. Als gevolg hiervan is er sprake van een chronisch verhoogd hartminuutvolume zonder dat dit extra cardiale inspanning kost. De toename in glomerulaire filtratie snelheid, die tegelijkertijd optreedt met de toename in hartminuutvolume, wordt verondersteld het gevolg te zijn van relaxatie van de afferente en efferente renale bloedvaten. De toename in linker atrium diameter, de afname in plasma renine concentratie tussen de 5e en $8 \mathrm{e}$ zwangerschapsweek en de stabilisering van de glomerulaire filtratie snelheid na de 8 e zwangerschapsweek kenmerken een toename in de vullingstoestand van het vaatbed, waarschijnlijk ter compensatie van een initiële situatie van (relatieve) hypovolemie als gevolg van de gegeneraliseerde vasorelaxatie. Het ontbreken van veranderingen in de dimensies van de vena cava inferior illustreert mogelijk dat de gepostuleerde (tijdelijke) wanverhouding tussen omvang en vullingstoestand van het vaatbed voornamelijk optreedt in arteriën vanwege hun hogere gehalte aan glad spierweefsel. Dientengevolge zijn de veranderingen in de vena cava inferior waarschijnlijk te gering om met onze technieken te kunnen worden opgemerkt.

Gebruik makend van het boven geschetste concept werden de data verkregen bij 6 vrouwen, die een zwangerschap doormaakten, die werd gecompliceerd door foetale groeivertraging, vergeleken met de data in de normale zwangerschap. Vergelijking van de cardiovasculaire gegevens makte duidelijk dat in de vroege 
zwangerschap deze abnormale groep verschilt van de referentie groep door een geringere toename in hartminuutvolume en door een consequent kleiner en geringer toenemende linker atrium diameter. De uitkomsten van de variabelen, die gerelateerd zijn met volume homeostase, konden deze aanwijzingen voor een verminderde volume expansie niet tegenspreken, maar slechts beperkt onderschrijven. Dit was deels het gevolg van de relatief grote variabiliteit in de metingen en deels door de kleine aantallen in de beide groepen. Gecombineerd vormen deze bevindingen een indicatie voor een inadequate vullingstoestand van het vaatbed al in de eerste weken van de zwangerschap, een situatie die voortduurt na de $8 \mathrm{e}$ zwangerschapsweek. Tijdens zwangerschappen, waarbij foetale groeivertraging optreedt, wordt in eerste instantie de geïnduceerde (relatieve) ondervullingstoestand niet adequaat gecompenseerd. Dit lijkt op te treden ondanks normale activatie van de volume retinerende mechanismen. Deze blijkbaar normale activatie pleit voor een adequate primaire stimulus. Aanwijzingen voor een vervolgens inadequate volume expansie vloeien ook voort uit het patroon van de veranderingen in de dimensies van de vena cava inferior in de abnormale groep. De afname van de minimale diameter van de vena cava inferior in de eerste zwangerschapsweken suggereert de mobilisatie van een deel van het statische bloedvolume. Dit fenomeen treedt normaal gesproken alleen op als er sprake is van een ondervullingstoestand van het vaatbed.

Tenslotte evalueerden we tevens postpartum in hoeverre de toestand van de circulatie buiten de zwangerschap verschilt bij vrouwen, die een (te) klein kind kregen, met vrouwen die een normaal groot kind kregen. Vrouwen in de eerste groep hadden, zonder dat er sprake was van demografische verschillen, een hyperdynamische circulatie. Dit uitte zich in een kleinere linker atrium diameter en een grotere cardiale contractiliteit. Het gebruik van postpartum gegevens beperkt echter de waarde van deze bevinding.

Samenvattend kunnen uit de resultaten van de studies in dit proefschrift de volgende conclusies worden getrokken:

1. veranderingen in de moederlijke hemodynamiek en volumehuishouding zijn al in de 5 e zwangerschapsweek aantoonbaar,

2. de eerste systemische verandering in de maternale hemodynamiek lijkt te bestaan uit een gegeneraliseerde, aspecifieke vaatverslapping,

3. in zwangerschappen, die gecompliceerd worden door foetale groeivertraging, verloopt de hemodynamische adaptatie in de vroege zwangerschap afwijkend. 
Chapter 12

\section{ZUSAMMEFASSIG UF BÄRNDÜTSCH}


We ne Frou ir guete Hoffnig isch, bruuchts I irem liib ganz Zilete vo Verändrige, für die nöi Ufgab möge z'bewältige. S'Bluet tuet angersch fliesse u stocke, u ou d'Niere, usi Bluetwösch, mues sy ghörig aapasse, u derzue no alles I de erschte drei Mönet.

We ne Muetter Problem het mit irem Bluetdruck, oder s'Chindli, wohl behüetet I irem Buuch, zweni tuet wachse, de pumpets Härz zweni Bluet dür d'Niere, d'Wöscherei ma nid nache, s'Bluet Isch nümme so suber wienes sötti sy. By söttige Müettere merkt me's scho z'mitte if Hoffnig, dass da öppis nümme tuet schtimme.

Zersch hei mr gschtudiert, gäb me mit der Hülf vo Echokardiogramme oder mit Schallwälle chörntl de Durchmässer vo der Grosse Hohlvene mässe, u demit Aagabe über Iri Füllig zfinge. Derna hel mer gsuecht usezfinge, wie sich die Wärte im Verlouf vo dene bsungere nühn Mönet tüege ändere.

Im zwöite Teil vo dere Dokterarbeit hei mer de das, was bi dene Mässige isch usecho, bis i ds chlinschte usbeinlet. De hei mier wiiter zäh jungi, gsundi Froue, alli ou ir Hoffnig, zuechezoge, u ou da gmässe, was sich i irem Bluetkreislauf u irer Bluetmängi tüegi ängere. Die Mässwärt hei mir derna als Grundlag gno, u ou gmerkt, dass die Verändrige vor allem vor füfte bis zur achte Wuche passiere. Dasch de grad die Zytperiode, wo mer ganz gründlich hei aaglueget, u ne Hüfigkeitsverteilig vo de Abwilchige ufgstellt hei.

Us all dene Mässige hei mir de Idruck übercho, dass da neime im Liib e Schtell sigi, bishär ubekannt, wo d'Schpannig I de Bluetgfäss ir Schwangerschaft laal la abnäh. Zum Usgliich müesi de s'Härz stränger pumpe, süsch täti ja s'Bluet sozäge versacke, d'Gfäss wäre ja nümme ir Ornig gfüllt.

Uf Grund vo dene Untersuechige u mit dr gliche Arbetswiis hei mir de d'Wärte vo sächs Froue aaglueget, wo nes äbe nid gschtimmt het, irl Chindli hei nid welle wachse wie nes sötti sy. Es hät sich de zeiget, dass iri Härze de Usglich, die Meharbeit nid hei zschtand braacht, iri Chindli sy underversorgt plibe. Me het das au dra gseh, dass iri lingge Vorhöf (vom Härz) nid grösser sy worde, u iri Bluetgfäss sy nid gfüllt gsy wienes hetti sölle sy.

Zum Schluss hei mr de no usgwärtet, gäb d'Bluetgfäss by Froue wo normalgrosse Ching hei gha, angers sige als die vo däne, wo n es chliises Bebe hei gebore. Natürli cha me das erscht na de Geburt mässe, u dermit nid glich wärte, aber öppis hei mer gseh: dass die Müettere mit ehnder z'chline Ching a grüseli läbhafte Kreislouf heige. 
Chapter 13

\section{LITERATURE}


Adams JQ Alexander AM.

Alterations in cardiovascular physiology during labor. Obstet Gynecol 1958;12:542-549.

Andros GJ, Arbor A.

Blood pressure in normal pregnancy. Am J Obstet Gynecol 1945;50:300-306.

Arias $F$.

Expansion of intravascular volume and fetal outcome in patients with chronic hypertension and pregnancy.

Am J Obstet Gynecol 1975;123:610-616.

Assali NS, Rauromo L, Peltonen T.

Measurement of uterine blood flow and uterine metabolism. Am J Obstet Gynecol 1960;79:86-98.

Atherton JC, Bulock D, Pirie S.

The effect of pseudopregnancy on glomerular filtration rate and salt and water reabsorption in the rat. J Physiol 1982;424:11-20.

Atkins AF, Watt JM, Milan P, Davies P, Seluyn Crawford J.

A longitudinal study of cardiovascular dynamic changes throughout pregnancy. Eur J Obstet Gynecol Reprod Biol 1981;12:225-233.

August $P$, Lenz $T$, Alex K, Druzin ML, Eldersbeim TG, Hutson M, Müller FB, Laragb JH, Sealey JE

Longitudinal study of the renin-angiotensin-aldosterone system in hypertensive pregnant women: deviations related to the development of superimposed preeclampsia. Am J Obstet Gynecol 1990;163:1612-1621.

Bader RA, Bader ME, Rose DJ, Braumwald E.

Hemodynamics at rest and during exercise in normal pregnancy as studied by cardiac catheterization. J Clin Invest 1955;34:1524-1536.

Barron WM, Scbreiber J, Lindbeimer MD.

Effect of ovarian steroids on osmoregulation and vasopressin secretion in the rat. Am J Physiol 1986;250:E352-E361.

Barron WM.

Volume homeostasis during pregnancy in the rat. Am J Kidney Dis 1987;9:296-302.

Barron WM, Lindbeimer MD.

Osmoregulation in pseudopregnant and prolactin-treated rats: comparison with normal gestation. Am J Physiol 1988;254:R478-R484.

Barron WM, Dürr JA, Scbrier RW, Lindbeimer MD

Role of hemodynamic factors in osmoregulatory alterations in rat pregnancy. Am J Physiol 1989;257:R909-R916.

Bartb JA.

Echokardiographie während der Schwangerschaft - Verlaufsuntersuchungen bei Primigravidae. Zentralbl Gynäkol 1990;112:1009-1016

Barwin BN, Roddie IC.

Venous distensibility during pregnancy determined by graded venous congestion. Am J Obstet Gynecol 1976;125:921-923.

Bateman BG, Kolp LA, Nunley WC, Felder R, Burkett B.

Subclinical pregnancy loss in clomiphene citrate-treated women. Fertil Steril 1992;57:25-27.

Baylis C, Icbikawa I, Willis WT, Wilson CB, Brenner BM.

Dynamics of glomerular ultrafiltration. IX. Effects of plasma protein concentration. Am J Physiol 1977;232:F58-F72.

Baylis C.

The mechanism of increased glomerular filtration rate in the twelve-day pregnant rat. J Physiol 1980;305:405-414.

Baylis C.

Glomerular ultrafiltration in the pseudopregnant rat. Am J Physiol 1982;243:F300-F305.

Baylis C, Munger $K$.

Persistence of maternal plasma volume expansion in midterm pregnant rats maintained on a zero sodium intake: evidence that early gestational volume expansion does not require renal sodium retention. Clin Exp Hypertens $1990 \mathrm{a} ; \mathrm{B9}: 237-247$. 
Baylis C, Davison JM.

The urinary system. In: Clinical physiology in obstetrics. Hytten F, Chamberlain G, eds. Oxford: Blackwell Scientific Publication, 1990b: 245-302.

Baylis PH.

Osmoregulation and control of vasopressin secretion in healthy humans. Am J Physiol 1987;253:R671-R678.

\section{Beilin LI. Cbu ZM.}

Nitric oxide-dependent and -independent mechanisms of vasodilation in pregnancy. J Hypertens 1993;11:S148-S149.

Beldekas JC, Smitb B, Gerstenfeld LC, Sonensbein GE, Franzblau C.

Effects of 17B-estradiol on the biosynthesis of collagen in cultured bovine aortic smooth muscle cells. Biochemistry $1981 ; 20: 2162-2167$.

Belfort M, Uys P, Dommisse J, Davey DA.

Haemodynamic changes in proteinuric hypertension: the effects of rapid volume expansion and vasodilator therapy. Br J Obstet Gynaecol 1989;96:643-641.

Benedetti TJ, Cotton DB, Read JL.

Hemodynamic observations in severe preeclampsia with a flow-directed pulmonary artery catheter. Am J Obstet Gynecol 1980;136:465-470.

Benedetti TJ, Kates $R$, Williams $V$.

Hemodynamic observations in severe preeclampsia complicated by pulmonary edema. Am J Obstet Gynecol $1985 ; 152: 330-334$.

Blekta M, Hlavaty V, Trmkova M, Bendl J, Bendova L, Cbytil $M$.

Volume of whole blood and absolute amount of serum proteins in the early stage of late toxemia of pregnancy.

Am J Obstet Gynecol 1970;106:10-13.

Boekkooi PF, Janssen BJA, Verkeste CM, Kaufman P, Peeter LLH.

Hemodynamic response to volume stress in awake late-pregnant and nonpregnant rats. Pediatr Res 1991;30:479-86.

Bond AL, August P, Druzin ML, Atlas SA, Sealey JE, Laragb JH.

Atrial natriuretic peptide in normal and hypertensive pregnancy. Am J Obstet Gynecol 1989;160:1112-1116.

Bouchard A, Blumlein S, Scbiller NB, Scblitt S, Byrd BF, Ports T, Chatterjee K.

Measurement of left ventricular stroke volume using continuous wave Doppler echocardiography of the ascending aorta and M-mode echocardiography of the aortic valve. J Am Coll Cardiol 1987;9:75-83.

Brebm $H$, Kindling $E$.

Der Kreislauf während Schwangerschaft und Wochenbett. Arch Gynäk 1955;185:696-712.

\section{Brenner BM, Ballermann BJ, Gunning ME, Zeidel ML.}

Diverse biological actions of atrial natriuretic peptide. Physiol Rev 1990;70:665-699.

\section{Brö́cher-Mortensen J, Rödbro P.}

Selection of routine method for determination of glomerular filtration rate in adult patients. Scand J Clin Lab Invest 1976;36:35-43.

Brosens I, Robertson WB, Dixon HG.

The physiological response of the vessels of the placental bed to normal pregnancy. J Pathol Bacteriol 1967;93:569579.

Brougbton Pipkin F, Oats. JN, Symonds EM.

Sequential changes in the human renin-angiotensin system following delivery. Br J Obstet Gynaecol 1978;85:821-827.

Brougbton Pipkin F, Morrison R, O'Brien PMS.

The effect of prostaglandin E1 upon the pressor and hormonal response to exogenous angiotensin II in human pregnancy. Clin Sci 1987;72:351-357.

\section{Brougbton Pipkin F.}

The renin-angiotensin system in normal and hypertensive pregnancies. In: Rubin PC, ed. Hypertension in pregnancy. Amsterdam: Elsevier Science Publishers, 1988: 118-151.

Brown D, Davies DL, Lever AF, Robertson JIS.

Variations in plasma renin during the menstrual cycle. Br Med J 1964;2:1114-1115. 
Brown MA, Sinosicb MJ, Saunders DM, Gallery EDM.

Potassium regulation and progesterone-aldosterone interrelationships in human pregnancy: a prospective study. Am J Obstet Gynecol 1986a;155:349-353.

Brown MA, Crawford GA, Horgan EA, Gallery EDM.

Arginine vasopressin in hypertensive human pregnancy. Clin Exp Hypertens 1986b;B5:253-269.

Brown MA, Nicbolson E, Ross MR, Norton HE, Gallery EDM.

Progressive re-setting of sodium-renin-aldosterone relationships in human pregnancy. Clin Exp Hypertens 1987;B5:349-374.

Brown MA, Brougbton Pipkin F, Symonds EM.

The effects of intravenous angiotensin II upon sodium and urate excretion in human pregnancy. J Hypertens 1988a;6:457-464.

Brown MA, Crawford GA, Horgan EA, Gallery EDM.

Arginine vasopressin in primigravid human pregnancy. A prospective study. J Reprod Med 1988b;33:35-40.

Brown MA, Zammit VC, Lowe SA.

Capillary permeability and extracellular fluid volumes in pregnancy-induced hypertension. Clin Sci 1989;77:599-604.

Brown MA, Adsett D.

Automated blood pressure recording in pregnancy. Clin Exp Hypertens 1991;B10:7-19.

Brown MA, Mitar DA, Wbitworth JA.

Measurement of plasma volume in pregnancy. Clin Sci 1992a;83:29-34.

Brown MA, Zammit VC, Mitar DM.

Extracellular fluid volumes in pregnancy-induced hypertension. J Hypertens 1992b;10:61-68.

Burg JR, Dodek A, Kloster FE, Metcalfe J.

Alterations of systolic time intervals during pregnancy. Circulation 1974;49:560-564.

Burvell CS, Strayborn WD.

Observations on the circulation during and after pregnancy. J Clin Invest 1933;12:977.

Burwell CS, Strayborn WD, Flickinger D, Corlette MB, Bowerman EP, Kennedy JA. Circulation during pregnancy. Arch Intern Med 1938;11:979-1003.

Campbell DM, MacGillivray 1 .

Comparison of maternal response in first and second pregnancies in relation to baby weight. J Obstet Gynaecol Br Commonw 1972;79:684-693.

Campbell DM, Haites $N$, MacLennan F, Rawles J.

Cardiac output in twin pregnancy. Acta Genet Med Gemellol (Roma) 1985a;34:225-228.

Campbell DM, Campbell AJ.

Arterial blood pressure - the pattern of change in twin pregnancies. Acta Genet Med Gemellol (Roma) 1985b; 34:217223.

Campos O, Andrade JL, Bocanegra J, Ambrose JA, Carvalbo AC, Harada $K$, Martinez EE.

Physiologic multivalvular regurgitation during pregnancy: a longitudinal Doppler echocardiographic study. Int J Cardiol 1993;40:265-272.

Cannella G, Albertini A, Assanelli D, Poiesi C, Sandrini M, Maiorca $R$.

Effects of changes in intravascular volume on atrial size and plasma levels of immunoreactive atrial natriuretic peptide in uremic man. Clin Nephrol 1988;30:187-192.

Canton AD, Conte G, Esposito C, Fuiano G, Guasco R, Russo D, Sabbatini M, Uccello F, Andreucci VE. Effects of pregnancy on glomerular dynamics: micropuncture study in the rat. Kidney Int 1982;22:608-612.

Capeless EL, Clapp JF.

Cardiovascular changes in early phase of pregnancy. Am J Obstet Gynecol 1989;161:1449-1453.

Capeless EL, Clapp JF.

When do cardiovascular parameters return to their preconception values? Am J Obstet Gynecol 1991;165:883-886. 
Castor LC, Arora C, Panez S, Panez H, Valenzuela G, Hobel CJ.

Plasma atrial natriuretic peptide levels during the rat estrous cycle, pregnancy and the puerperium. Am J Obstet Gynecol 1989;160:15-19.

Caton D, Banner TE.

Doppler estimates of cardiac output during pregnancy. Bull N Y Acad Med 1987;63:727-731.

Caton WL, Roby CC, Reid DE, Caswell R, Maletskos CJ, Flubarty RS, Gibson JG.

The circulating red cell volume and body haematocrit in normal pregnancy and the puerperium. Am J Obstet Gynecol 1951;61:1207-1217.

Cba SC, Aberdeen GW, Nuwaybid BS, Quillen EW.

Influence of pregnancy on mean systemic filling pressure and the cardiac function curve in guinea pigs. Can J Physiol Pharmacol 1991;70:669-674.

Cberiex EC, Leunissen KML, Janssen JHA, Mooy JMV, Van Hooff JP.

Echography of the inferior vena cava is a simple and reliable tool for estimation of "dry weight" in haemodialysis patients. Nephrol Dial Transplant 1989;4:563-568.

Cbesley LC, Valenti C, Uichango L.

Alterations in body fluid compartments and exchangeable sodium in the early puerperium. Am J Obstet Gynecol 1959;77:1054-1064.

Chesley LC, Sloan DM.

The effect of posture on renal function in late pregnancy. Am J Obstet Gynecol 1964;89:754-759.

Chesley LC.

Plasma and red cell volumes during pregnancy. Am J Obstet Gynecol 1972;112:440-450.

Clapp JF, Seawand BL, Sleamaker RH, Hiser J.

Maternal physiologic adaptations to early human pregnancy. Am J Obstet Gynecol 1988;159:1456-1460.

Clark SL, Greenspoon JS, Aldabl D, Pbelan JP.

Severe preeclampsia with persistent oliguria: management of hemodynamic subsets. Am J Obstet Gynecol 1986;154:490-494.

Clark SL, Cotton DB.

Clinical indications for pulmonary artery catheterization in the patient with severe preeclampsia. Am J Obstet Gynecol 1988;158:453-458.

Clark SL, Cotton DB, Lee W, Bishop C, Hill T, Soutbwick J, Pivamik J, Spillman T, DeVore GR, Pbelan J, Hankins $G D V$, Benedetti TJ, Tolley D.

Central hemodynamic assessment of normal term pregnancy. Am J Obstet Gynecol 1989;161:1439-1442.

Clark SL, Cotton DB, Pivarnik JM, Lee W, Hankins GDV, Benedetti TJ, Pbelan JP.

Position change and central hemodynamic profile during normal third-trimester pregnancy and post partum. Am J Obstet Gynaecol 1991;164:883-887.

Cloenen SE, Lippert TH.

Effect of plasma expanders in toxemia of pregnancy. N Engl J Med 1972;ii:1356-1357.

Conrad KP, Colpoys MC.

Evidence against the hypothesis that prostaglandins are the vasodepressor agents of pregnancy: serial studies in chronically instrumented conscious rats. J Clin Invest 1986;77:236-245.

Conradsson TB, Werko $L$.

Management of heart disease in pregnancy. Prog Cardiovasc Dis 1974;16:407-419.

Cotton DB, Lee W, Hubta JC, Dorman $K F$.

Hemodynamic profile of severe pregnancy-induced hypertension. Am J Obstet Gynecol 1988;158:523-529.

Coulam CB.

Association between infertility and spontaneous abortion. Am J Reprod Immunol 1992;27:128-129.

Cournand A, Ranges HA.

Catheterization of the right auricle in man. Proc Soc Exp Biol Med 1941;46:462-466. 
Creasy RK, Barrett CT, DeSwiet M, Kabanpaa KV, Rudolpb AM.

Experimental intrauterine growth retardation in the sheep. Am J Obstet Gynecol 1972;112:566-573.

Croall J, Sberrif S, Matthews J,

Non-pregnant maternal plasma volume and fetal growth retardation. Br J Obstet Gynaecol 1978;85:90-95.

Cunningham FG, Lindbeimer $M D$.

Hypertension in pregnancy. N Engl J Med 1992;326:927-932.

Curien ND, Merillon IP, Prasquier $R$, Vervin $P$, Goungon $R$.

Modifications de la relation pression-volume passive ventriculaire gauche lors d'une expansion volumique aigue. Arch Mal Coeur 1983;76:759-770.

Daniel MK, Bennett B, Dawson AA, Rawles JM.

Haemoglobin concentration and lineair cardiac output, peripheral resistance, and oxygen transport. Br Med J $1986 ; 292: 923-926$.

Daunter $B$.

Immunology of pregnancy: towards a unifying hypothesis. Eur J Obstet Gynecol Reprod Biol 1992;43:81-95.

Davies $M$

Polygeline. Dev Biol Stand 1987;67:129-131.

Davies P, Francis RI, Docker MF, Watt JM, Crawford JS.

Analysis of impedance cardiography longitudinally applied in pregnancy. Br J Obstet Gynaecol 1986;93:717-720.

Davis LE, Hobimer AR, Giraud GD, Paul MS, Morton MJ.

Vascular pressure-volume relationships in pregnant and estrogen-treated guinea pigs. Am J Physiol 1989;257:R1205R1211.

Davison JM, Hytten FE.

Glomerular filtration during and after pregnancy. J Obstet Gynaecol Br Commonw 1974;81:588-595.

Davison JM, Dunlop W.

Renal hemodynamics and tubular function in normal human pregnancy. Kidney Int 1980;18:152-161.

Davison JM, Noble MC.

Serial changes in 24-hour creatinine clearance during normal menstrual cycles and the first trimester of pregnancy. Br J Obstet Gynaecol 1981a;88:10-17.

\section{Davison JM, Valloton MB, Lindbeimer MD.}

Plasma osmolality and urinary concentration and dilution during and after pregnancy: evidence that lateral recumbuncy inhibits maximal urinary concentrating ability. Br J Obstet Gynaecol 1981b;88:472-479.

Davison JM.

Renal haemodynamics and volume homeostasis in pregnancy. Scand J Clin Lab Invest (Suppl) 1984a;169:15-27.

Davison JM, Gilmore EA, Dürr J, Roberson GL, Lindbeimer MD.

Altered osmotic thresholds for vasopressin secretion and thirst in human pregnancy. Am J Physiol 1984b;246:F105F109.

Davison JM.

Overview: kidney function in pregnant women. Am J Kid Dis 1987;4:248-252.

Davison JM, Sbiells EA, Pbilips PR, Lindbeimer MD.

Serial evaluation of vasopressin release and thirst in human pregnancy. Role of human chorionic gonadotrophin in the osmoregulatory changes of gestation. J Clin Invest 1988;81:798-806.

Davison JM, Sbiells EA, Barron $W M$, Robinson AG, Lindbeimer MD.

Changes in the metabolic clearance of vasopressin and of plasma vasopressinase throughout human pregnancy.

J Clin Invest 1989;83:1313-1318.

De Alvarez RR, Bratuold GE.

Renal glomerulotubular mechanisms during normal pregnancy. Am J Obstet Gynecol 1958;75:931-944.

De Swiet $M$, Talbert DG.

The measurement of cardiac output by electrical impedance plethysmography. Are the assumptions valid? Br J Obstet Gynaecol 1986; 93:721-726. 
De Vries PJF, Tyssen CM, Struyker-Boudier HAJ, Smits JFM.

Atrial natriuretic factor increases albumin extravasation in conscious rats. Pflugers Arch 1990;415:507-509.

Deen $W M$, Robertson CR, Brenner BM.

A model of glomerular ultrafiltration in the rat. Am J Physiol 1972;223:1178-1183.

Degani S, Abinader E, Lewinsky $R$, Sbapiro I, Sbarf $M$.

Maternal echocardiography in hypertensive pregnancies. Gynecol Obstet Invest 1989;27:2-5.

Dekker GA, Sibai BM.

Early detection of preeclampsia. Am J Obstet Gynecol 1991;165:160-172.

DeVane GW,

Vasopressin levels during pregnancy and labor. J Reprod Med 1985;30:324-327.

Devereux RB, Reicbek $N$.

Echocardiographic determination of the left ventricular mass in man. Anatomic validation of the method. Circulation 1977;55:613-618.

Di Donato M, Mori F, Barletta G, Dabizzi RP, Fantini F.

Effect of Dextran infusion on left atrial size in normal subjects. Cardiology 1982;69:257-264.

Douglas BH, Harlan JC, Langford HG, Richardson TQ.

Effect of hypervolemia and elevated arterial pressure on circulatory dynamics of pregnant animals. Am J Obstet Gynecol 1967;98:889-894.

Dubois D, Dubois EF.

A formula to estimate the approximate surface area if height and weight be known. Arch Intern Med (Chicago) 1916;17:863.

Duffus GM, MacGillivray I, Dennis KJ.

The relationship between baby weight and changes in maternal weight, total body water, plasma volume, electrolytes and proteins, and urinary oestriol excretion. J Obstet Gynaecol Br Commonw 1971;78:97-104.

Duffus GM, MacGillivray I.

Plasma and red cell volumes during pregnancy. Am J Obstet Gynecol 1972;112:440-450.

Dunlop W.

Serial changes in renal haemodynamics during normal human pregnancy. Br J Obstet Gynaecol 1980;88:1-9.

Dunne FP, Barry DG, Ferriss JB, Grealy G, Murpby D.

Changes in blood pressure during the normal menstrual cycle. Clin Sci 1991;81:515-518.

Duprez D, Kaufman JM, Liu YR, Van Hoecke J, Van Trappen Y, Tbiery M, Vermeulen A, Clement D.

Increases in atrial natriuretic peptide after delivery and in the puerperium. Am J Cardiol 1989;64:674-675.

Duvekot IJ, Cheriex EC, Pieters FAA, Peeters LLH.

The development of IUGR and/or preeclampsia is preceded by defective maternal cardiovascular adaptation in early pregnancy. Proceedings of the 39th Annual Meeting of the Society for Gynecologic Investigation; 1992 Mar 18-21; San Antonio. Abstract No, 129.

Duvekot II, Cberiex EC, Pieters FAA, Menbeere PPCA, Peeters LLH.

Early-pregnancy changes in hemodynamics and volume homeostasis are consecutive adjustments triggered by a primary fall in systemic vascular tone. Am J Obstet Gynecol 1993a;169:1382-1392.

Duvekot II, Cheriex EC, Pieters FAA, Menbeere PPCA, Peeter LLH.

Nonpregnant hyperdynamic cardiac function predisposes to the development of intra-uterine growth retardation. Clin Exp Hypertens 1993b;12:388 (abstract).

Duvekot J], Cheriex EC, Tan WD, Heidendal GAK, Peeters LLH.

Volume-dependent echocardiographic parameters are unsuitable for estimating baseline blood volume but are useful for detecting acute changes in vascular filling state. Basic Res Cardiol 1994a;89:270-277.

Duvekot IJ, Cheriex EC, Heidendal GAK, Tan WD, Peeters LLH.

Measurement of anterior-posterior diameter of inferior vena cava by ultrasonography: a new non-invasive method to assess acute changes in vascular filling state. Cardiovasc Res 1994b;28:1269-1272. 
Easterling TR, Watts $H$, Scbmucker BC, Benedetti TJ.

Measurement of cardiac output during pregnancy: validation of Doppler technique and clinical observations in preeclampsia. Obstet Gynecol 1987;69:845-850.

Easterling TR, Benedetti TJ, Carlson KL, Watts DH.

Measurement of cardiac output in pregnancy by thermodilution and impedance techniques. $\mathrm{Br} \mathrm{J}$ Obstet Gynaecol 1989a;96:67-69.

Easterling TR, Benedetti TJ.

Preeclampsia: a hyperdynamic disease model. Am J Obstet Gynecol 1989b;160:1447-1453.

Easterling TR, Benedetti TJ.

Measurement of cardiac output by impedance technique. Letter to the editor. Am J Obstet Gynecol 1990a;163: 1105-1107.

Easterling TR, Carlson KI, Scbmucker BC, Brateng DA, Benedetti TJ.

Measurement of cardiac output in pregnancy by Doppler technique. Am J Perinatol 1990b;7:220-222.

Easterling TR, Benedetti TJ, Scbmucker BC, Millard SP.

Maternal hemodynamics in normal and preeclamptic pregnancies: a longitudinal study. Obstet Gynecol 1990c;76:1061-1069.

Easterling TR, Benedetti TJ, Scbmucker BC, Carlson K, Millard SP.

Maternal hemodynamics and aortic diameter in normal and hypertensive pregnancies. Obstet Gynecol 1991; 78:1073-1077.

Eskes TKAB, Weyer A, Kramer $N$, Van Elteren $P$.

Arterial blood pressure and posture during pregnancy. Eur J Obstet Gynecol Reprod Biol 1974;4:87-94.

Exalto $N$.

De jonge zwangerschap [Dissertation]. Nijmegen: Katholieke Universiteit Nijmegen, 1979.

Fay TN, Grudzinskas JG.

Human endometrial peptides: a review of their potential role in implantation and placentation. Hum Reprod 1991;6:1311-1326.

Feldscbub J, Enson Y.

Prediction of normal blood volume, relation of blood volume to body habitus. Circulation 1977;56:605-612.

Fievet P, Pleskov L, Desailly I, Carayon A, De Fremont JF, Coevoet B, Comoy E, Demory JE, Verboest $P$, Boulanger JC. Plasma renin activity, blood uric acid and plasma volume in pregnancy-induced hypertension. Nephron 1985; $40: 429-432$.

Finn WL, Tunny TJ, Klemm SA, Jones IS, De Voss K, Gordon RD.

Sodium and volume dysregulation after apparently normal pregnancy is suggested by abnormal levels of atrial natriuretic peptide, renin and aldosterone. Clin Exp Pharmacol Physiol 1991;18:269-273.

Flessas AP, Ryan TJ.

Left ventricular diastolic capacity in man. Circulation 1982;65:1197-1203.

Forman RG, Fries $N$, Testart J, Belaisch-Allart J, Hazout A, Frydman $R$.

Evidence for an adverse effect of elevated serum estradiol concentrations on embryo implantation. Fertil Steril $1988 ; 49: 118-122$.

Forman RG, Eycbenne B, Nessmann C, Fydman R, Robel $P$.

Assessing the early luteal phase in in vitro fertilization cycles: relationships between plasma steroids, endometrial receptors and endometrial histology. Fertil Steril 1989;51:310-316.

Fortuin NJ, Hood WP, Craige E.

Evaluation of left ventricular function by echocardiography. Circulation 1972;46:26-35.

Foumier A, Gregoine I, El Esper N, Lalau JD, Westeel PF, Makdassi R, Fievet P,

De Bold AJ. Atrial natriuretic factor in pregnancy and pregnancy-induced hypertension. Can J Physiol Pharmacol 1991;69:1601-1608.

Franx A, Van der Post JAM, Elfering IM, Veerman DP, Merkus HMWM, Boer K, Van Montfrans GA.

Validation of automated blood pressure recording in pregnancy. Br J Obstet Gynaecol 1994;101:66-69. 


\section{Easterling TR, Watts $H$, Scbmucker BC, Benedetti T].}

Measurement of cardiac output during pregnancy: validation of Doppler technique and clinical observations in
preeclampsia. Obstet Gynecol 1987;69:845-850. Easterling TR, Benedetti T], Carlson $K L$, Watts $D H$

Measurement of cardiac output in pregnancy by thermodilution and impedance techniques. Br J Obstet Gynaecol
1989a-96-67-69.

Easterling TR, Benedetti TT.

Easterling $T R, B$ Benedetti $ᄁ$.
Measurement of cardiac ou

Measurement of cardiac output by impedance technique. Letter to the editor. Am J Obstet Gynecol 1990a; 163 :
11155-1107.

Easterling TR, Carlson KI, Scbmucker BC, Brateng DA, Benedetti T].

ment of cardiac output in pregnancy by Doppler technique. Am J Perinatol 1990b; 7:220-22

Easterling TR, Benedetti T, Scbmucker BC, Millard SP.

Maternal hemodynamics in normal and preeclamptic pregnancies: a longitudinal study. Obstet Gynecol
1990c; 76 : 1061-1069.

Easterling $T R$, Benedetti TJ, Scbmucker $B C$ C Carlson $K$, Millard $S P$
Maternal hemodynamics and aortic diameter in

Maternal hemodynamics and aoric diameter in normal and hypertensive pregnancies. Obstet Gynecol 1991,
78:1073-1077.

Eskes TKAB, Weyer A, Kramer N, Van Elteren $P$.

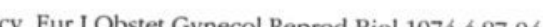

Exalto $N$.

De jonge zwangerschap [Dissertation]. Nijmegen: Katholieke Universiteit Nïmegen, 1979.

Fay TN, Grudzinskas JG

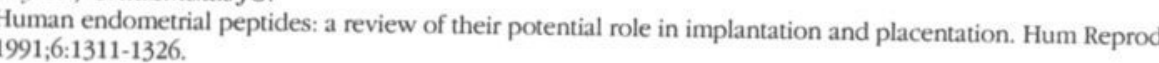

Feldschub

Prediction of normal blood volume, relation of blood volume to body habitus. Circulation 1977:56:605-612. Fiever $P$, Pleskow L Desailly I, Carazon A, De Fremont JF, Coevoet B, Comoy E, Demory JE, Verboest $P$, Boulanger $J C$.
Plasma renin activity, blood uric acid and plasma volume in pregnancy-induced hypertension. Nephron
$1085 ; 40: 429-432$

Finn Wh, Tunny TJ, Klemm SA, Jones IS, De Vass $K$, Gordon RD.

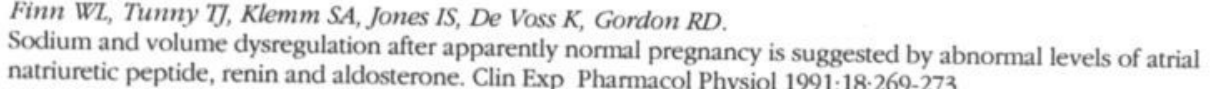

Flessas $A P$, Ryan $T$ T.
Left ventricular diasto

Forman RG, Fries $N$, Testar J., Belaiscb-Allar J., Hazout A, Fndman R.

Evidence for an adverse effect of elevated serum estradiol concentrations on embryo implantation. Fertil Steril
1988;49:118-122.

Forman $R G$, Eycbenne B, Nessmann C, Fydman R, Robel $P$.

Aorman $R G$, Ejcbenne B, Nessmann $C$, Findman $R, R$ Robel $P$.
Assessing the early luteal phase in in vitro fertilization cycles: relationships between plasma steroids, endometrial
receptors and endometrial histology. Ferril Steril 1989;51:310-316.

Fortuin $N$, Hood $W P$, Craige $E$.
Evaluation of left ventricular function

Fournier A, Gregoire I, El Esper N, Lalau JD, Westeel PF, Makdassi R, Fievet P

1991;69:1601-1608.

Franx A, Van der Post JAM, Elfering IM, Veerman DP, Merreus HMWM, Boer $K$, Van Montrans GA.
Validation of automated blood pressure recording in pregnancy. Br J Obstet Gynaecol 1994; $101: 66-69$.
Friedman SA.
Preeclampsia: a review of the role of prostaglandins. Obstet Gynecol 1988;71:122-137.

Froblich ED, Grim C, Labartbe DR, Maxuell MH, Perloff D, Weidman W.

Recommendations for human blood pressure determination by sphygmomanometers: report of a special tast force

Fukui $M$, Shigemi $K$ K.
Determination of single and repeated red cell volumes by the indicator dilution method using carbon monoxide as

Fullerton WT, Hytten FE, Klopper AI, MCKay E.
A case of quadruplet pregnancy. J Obstet Gynaecol Br Commonw 1965;72:791-796.

Gallery $E D M$, Hunyor $S N$, Ross $M$, Gyory $A A$.
Predicting the development of pregnancy-associated hypertension. The place of standardised blood-pressure measGallery EDM, Hunyor SN, Gyôry AZ

Thema volume contraction: a significant factor in both pregnancy-associated hypertension (preeclampsia) and chroGallery EDM, Delprado W, Gyón AZ.

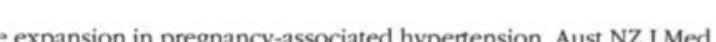

Gallery EDM, Brown MA

Gardin JM, Tobis JM, Dabestani A, Smith C, Ellkayam U, Castleman E, Wbite D, Allfie A, Henry WL.

Superionity of 2-D measurement of aortic vessel diameter in Doppler echocardiographic estimates of left ventricular

Gibson $H M$

Plasma volume and glomenular filtration rate in pregnancy and their relation to differences in fetal growth. J Obstet 1973;80:1067-1074-10

Am J Physiol 1992; 2633 :H1911-H1918.

Goodlin $R C$, Quaife $M A$, Dirksen $J W$.
The significance, diagnosis and treatment of maternal hypovolemia as associated with fetal/maternal illness. Semin

Goodlin $R C$, Anderson $J$ C, Gallagber TE.
Relationship between amniotic fluid volum

elationship between amniotic fluid volume and matemal plasma volume expansion. Am J Obstet Gynecol

Goodlin RC, Dobry CA, Anderson JC, Woods RE, Quaife M.

Clinical signs of normal plasma volume expansion during pregnancy. Am J Obstet Gynecol 1983b; 145:1001-1007. Gynecol 1984;148:224-22

Goodrich SM, Wood II

Peripheral venous distensibility and velocity of venous blood flow during pregnancy or oral contraceptive therapy.

Greer IA.
Ambulatory blood pressure in pregnancy: measurement and machines. BrJ Obstet Gynaecol 1993;100:887-889.

Greenendijik R, Trimbos JBMJ, Wallenburg HCS.
Hemodynamic measurements in preeclampsia. Am J Obstet Gynecol 1984;150:232-236

Grollman A

in health and disease. London: Ballière, Tindall and Cox, 1932.

Grunewald C, Nisell H, Carlstrom K, Kublickas M, Randmaa I, Nylund

cute volume expansion in normal pregnancy and preeclampsia : effects on plasma atrial natriuretic peptide (ANP) 
and cyclic guanosine monophosphate (cGMP) concentrations and feto-maternal circulation. Acta Obstet Gynecol Scand 1994;73:294-299.

Guyton AC, Abernatby JB, Langston JB, Kaufmann BN, Faircbild HM.

Relative importance of venous and arterial resistances in controlling venous return and cardiac output. Am J Physiol 1959;196:1008-1015.

Guyton AC, Jones CE, Coleman TG.

Effect of right atrial pressure on venous return - the normal venous return curve. In: Guyton AC, ed. Circulatory physiology. Philadelphia: Saunders, 1973a: 188-204.

Guyton AC, Jones CE, Coleman TG.

Mean circulatory filling pressure, mean systemic pressure, and mean pulmonary pressure and their effect on venous return. In: Guyton AC, ed. Circulatory physiology. Philadelphia: Saunders, 1973b: 205-221.

Guyton AC, Jones CE, Coleman TG.

Effect of blood volume changes and orthostatic factors on cardiac output. In: Guyton AC, ed. Circulatory physiology. Philadelphia: Saunders, 1973c: 353-371.

Guyton AC.

Cardiac output, venous return and their regulation. In: Guyton AC, ed. Textbook of medical physiology. Philadelphia: Saunders, 1986a: 272-286.

Guyton AC.

The pulmonary circulation. In: Guyton AC, ed. Textbook of medical physiology. Philadelphia: Saunders, 1986b: 287-294.

Guyton AC.

Regulation of blood volume, extracellular fluid volume, and extracellular fluid composition by the kidneys and by the thirst mechanism. In: Guyton AC, ed. Textbook of medical physiology. Philadelphia: Saunders, 1986c: $425-437$.

\section{Hamilton HFH.}

The cardiac output in normal pregnancy. As determined by the Cournand right heart catheterisation technique. J

Obstet Gynaecol Br Empire 1949;56:548-552.

Hart MV, Hosenpud JD, Hobimer R, Morton MJ.

Hemodynamics during pregnancy and sex steroid administration in guinea pigs. Am J Physiol 1985;249:R179-R185.

Hart MV, Morton MJ, Hosenpud JD, Metcalfe J.

Aortic function during normal human pregnancy. Am J Obstet Gynecol 1986;154:887-891.

Hassan AAK, Carter G, Tooke JE.

Postural vasoconstriction in women during the normal menstrual cycle. Clin Sci 1990;78:39-47.

Hatjis CG, Greelisb JP, Kofinas AD, Stroud A, Hasbimoto K, Rose JC.

Atrial natriuretic factor maternal and fetal concentrations in severe preeclampsia. Am J Obstet Gynecol 1989;161:1015-1019.

Hatjis CG, Kofinas AD, Greelish JP, Swain M, Rose JC.

Atrial natriuretic factor concentrations during pregnancy and in the postpartum period. Am J Perinatol 1992;9:

275-278.

Hays PM, Cruiksbank DP, Dunn LJ.

Plasma volume determination in normal and preeclamptic patients. Am J Obstet Gynecol 1985;151:958-966.

Hedbeng E, Radbeng C.

Maternal heart volume and prematurity. Acta Obstet Gynecol Scand 1962;41:48-56.

\section{Heilmann L, Siekmann U.}

Hemodynamic and hemorheological profiles in women with proteinuric hypertension of pregnancy and in pregnant controls. Arch Gynecol Obstet 1989;246:159-168.

Heilmann L, Lobr M, Hintereicber M, Tempelboff G, Ose C.

Veränderungen der zentralen Hämodynamik und der peripheren Zirkulation in der Schwangerschaft. Geburtshilfe Frauenheilkd 1993;53:472-478.

Hendricks CH, Quilligan EJ.

Cardiac output during labor. Am J Obstet Gynecol 1956;71:953-972. 
Hirai $N$, Yanaibara T, Nakayama T, Isbibasbi M, Yamaji T.

Plasma levels of atrial natriuretic peptide during normal pregnancy and in pregnancy complicated by hypertension. Am J Obstet Gynecol 1988;159:27-31.

Hirata T, Wolfe SB, Popp RL, Helman CH, Feigenbaun $H$.

Estimation of left atrial size using ultrasound. Am Heart J 1969;78:43-52.

Hjertberg $R$, Belfrage $P$, Hagnevik $K$.

Hemodynamic measurements with Swan-Ganz catheter in women with severe proteinuric gestational hypertension (preeclampsia). Acta Obstet Gynecol Scand 1991;70:193-198.

Högberg $U$, Innala E, Sandström A.

Maternal mortality in Sweden, 1980-1988. Obstet Gynecol 1994;84:240-244.

Hollenberg $N K$.

The renin-angiotensin system and sodium homeostasis. J Cardiovasc Pharm 1984;6:S176-S183.

Huisman A, Aarnoudse JG.

Increased 2 nd trimester hemoglobin concentration in pregnancies later complicated by hypertension and growth retardation. Acta Obstet Gynecol Scand 1986;65:605-608.

Hunter S, Robson SC.

Adaptation of the maternal heart in pregnancy. Br Heart J 1992;68:540-543.

Huntsman LL, Stewart DK, Barnes SR, Franklin SB, Colocousis JS, Hessel EA.

Noninvasive Doppler determination of cardiac output in man: clinical validation. Circulation 1983;67:593-602.

Hussain SY, O'Brien PMS, De Souza V, Okonofua F, Dandona P.

Reduceci atrial natriuretic peptide concentrations in premenstrual syndrome. Br J Obstet Gynaecol 1990;97:397-401.

Hytten FE, Paintin DB.

Increase in plasma volume during normal pregnancy. J Obstet Gynaecol Br Commonw 1963;70:402-407.

Hytten FE.

Metabolic adaptations of pregnancy. In: Turnbull AC, Woodford FP, eds. Prevention of handicap through antenatal care. Amsterdam: Elsevier/North Holland, 1976: 35-39.

Iblen H, Amlie JP, Dale J, Forfang K, Nitter-Hauge S, Otterstad JE, Simonsen S, Mybre E.

Determination of cardiac output by Doppler echocardiography. Br Heart J 1984;51:54-60.

Jadoul FAC, Brougbton Pipkin F, Lamming GD.

Changes in the renin-angiotensin-aldosterone system in normotensive primigravidae in the four days after normal spontaneous delivery. Br J Obstet Gynaecol 1982;89:633-639.

James CF, Banner T, Levelle JP, Caton D.

Noninvasive determination of cardiac output throughout pregnancy. Anesthesiology 1985;63:A434.

James CF, Banner T, Caton D.

Cardiac output in women undergoing cesarian section with epidural or general anesthesia. Am J Obstet Gynecol 1989;160:1178-1184.

Jensen LK, Svanegaard J, Husby $H$.

Atrial natriuretic peptide during the menstrual cycle. Am J Obstet Gynecol 1989;161:951-952.

Jobenning AR, Barron WM.

Indirect blood pressure measurement in pregnancy: Korotkoff phase 4 versus phase 5. Am J Obstet Gynecol 1992;167:577-580.

Jobnson MR, Riddle AF, Grudzinskas JG, Sharma V, Collins WP, Nicolaides $K H$.

Reduced circulating placental protein concentrations during the first trimester are associated with preterm labour and low birth weight. Hum Reprod 1993;8:1942-1947.

Kalousek G, Havacek C, Nedoss B, Pollak VE.

Circadian rhythms of creatinine and electrolyte excretion in healthy pregnant women. Am J Obstet Gynecol $1969 ; 103: 856-867$.

Karlberg BE, Ryden $G$, Wichman $K$.

Changes in the renin-angiotensin-aldosterone and kallikrein-kinin systems during normal and hypertensive pregnancy.

Acta Obstet Gynecol Scand (Suppl) 1984; 118:17-24. 
Kass DA, Lowell Maugban W, Mao Guo Z, Kono A, Sunagawa K, Sagawa K.

Comparative influence of load versus inotropic states on indexes of ventricular contractility: experimental and theoretical analysis based on pressure-volume relationships. Circulation 1987;76:1422-1436.

Katz AM.

Endothelium-derived relaxin factor. J Am Coll Cardiol 1988;12:797-806.

Katz $R$, Karliner JS, Resink $R$.

Effects of a natural volume overload state (pregnancy) on left ventricular performance in normal human subjects. Circulation 1978;58:434-441.

$\operatorname{Kerr} M G$, Scott $D B$,

Samuel E. Studies of the inferior vena cava in late pregnancy. Br Med J 1964;i:532-533.

Kessler KM, Warde DAL, Ledis JE, Kessler RM.

Left ventricular size and function in women receiving oral contraceptives. Obstet Gynecol 1980;55:211-214.

Keyes J, Carey J, Mosses D, Beierwaltes W.

Manual of Nuclear Medicine Procedures. Cleveland: CRS Press, 1973: 95-98.

Kbong TY, De Wolf F, Robertson WB, Brosens I.

Inadequate maternal vascular response to placentation in pregnancies complicated by pre-eclampsia and by smallfor-gestational age infants. Br J Obstet Gynaecol 1986;93:1049-1059.

Kinney EL, Cortada X, Ventura R.

Cardiac size and motion during water immersion: implications for volume homeostasis. Am Heart J 1987;113:345-349.

Kinsella SM, Lee A, Spencer JAD.

Maternal and fetal effects on the supine and pelvic tilt positions in late pregnancy. Eur J Obstet Gynecol Reprod Biol 1990;36:11-17

Kircher BI, Himelman RB, Scbiller NB.

Noninvasive estimation of right atrial pressure from the inspiratory collapse of the inferior vena cava. Am J Cardiol 1990;66:493-496.

Kjedsen J.

Haemodynamic investigations during labour and delivery. Acta Obstet Gynecol Scand (Suppl) 1979;89:144-153.

Kloosterman GJ.

On intrauterine growth. Int J Gynaecol Obstet 1970;8:895-912.

Koller $O$.

The clinical significance of hemodilution during pregnancy. Obstet Gynecol Surv 1982;37:649-652.

Knutzen E, Olofsson P, Back SE, Nilsson-Eble P.

Glomerular filtration rate in pregnancy: a study in normal subjects and in patients with hypertension, preeclampsia and diabetes. Scand J Clin Lab Invest 1992;52:387-392.

Kubicek WG, Karnegis JN, Patterson RP, Witsoe DA, Mattson RH.

Development and evaluation of an impedance cardiac output system. Aerospace Medicine 1966;37:1208-1212.

Kuzniar J, Piela A, Sokret A.

Left ventricular function in preeclamptic patients: an echocardiographic study. Am J Obstet Gynecol 1983;146: 400-405.

Kuzniar J, Piela A, Skret A, Palczak R, Splawinski J, Micbna M.

Hemodynamic profile of mild pregnancy induced hypertension. Clin Exp Hypertens 1992;B11:131-146.

La Sala GB, Gaddi O, Bruno G, Brandi L.

Noninvasive evaluation of cardiovascular hemodynamics during multiple follicular stimulation, late luteal phase and early pregnancy. Fertil Steril 1989;51:796-802.

Laird NM, Ware JH.

Random effects models for longitudinal data. Biometrics 1982;38:963-974.

Laind-Meeter $K$, van de Ley $G$, Bom $T H$, Wladiminoff $J W$.

Cardiocirculatory adjustments during pregnancy - an echocardiographic study. Clin Cardiol 1979;2:328-332. 
Landesman $R$, Miller MM.

Blood volume changes during the immediate postpartum period. Obstet Gynecol 1963;21:40-48.

Landon MJ, Cspas DK, Sbiells EA, Davison JM.

Degradation of radiolabeled arginine vasopressin ( ${ }^{12 \mathrm{I}-\mathrm{AVP})}$ by the human placenta perfused in vitro. Br J Obstet Gynaecol 1988;95:488-492.

Lang RM, Pridjian G, Feldman T, Neumann A, Lindbeimer MD, Borrow KM.

Left ventricular mechanics in preeclampsia. Am Heart J 1991;121:1768-1775.

Larkin H, Gallery EDM, Hunyor SN, Gyory AZ, Boyce ES.

Cardiac and haemodynamic measurements in hypertensive pregnancy. Clin Sci 1980;59:357s-360s.

Ledoux F, Genest J, Nowaczynski W, Kucbel O, Lebel M.

Plasma progesterone and aldosterone in pregnancy. Can Med Assoc J 1975;112:943-947.

Leduc L, Wasserstrum N, Spillman T, Cotton DB.

Baroreflex function in normal pregnancy. Am J Obstet Gynecol 1991;165:886-890.

Lee W, Rokey $R$, Cotton $D B$.

Noninvasive maternal stroke volume and cardiac output determinations by pulsed Doppler echocardiography. Am J Obstet Gynecol 1988;158:505-510.

Lees $M M$, Taylor SH, Scott DB, Kerr MG.

A study of cardiac output at rest throughout pregnancy. J Obstet Gynaecol Br Commonw 1967;74:319-328.

Letsky $E$.

The haematological system. In: Clinical physiology in obstetrics. Hytten F, Chamberlain G, eds. Oxford: Blackwell Scientific Publication, 1990: 39-82.

Liley $A W$.

Clinical and laboratory significance of variations in maternal plasma volume in pregnancy. Int J Gynaecol Obstet 1970;8:358-362.

Lim YL, Walter WAW.

Systolic time intervals in normotensive and hypertensive human pregnancy. Am J Obstet Gynecol 1976;126:26-32.

Lindbard $J$.

Uber das Minutenvolume des Herzens bei Ruhe und bei Muskelarbeit. Pflugers Arch 1915;161:223-253.

Lindbeimer $M D$, Weston $P V$.

Effects of hypotonic expansion on sodium, water and urea excretion in late pregnancy: The influence of posture on these results. J Clin Invest 1969;48:947-956.

Lindbeimer MD, Barron WM, Davison JM.

Osmotic and volume control of vasopressin release in pregnancy. Am J Kidney Dis 1991a;17:105-111.

Lindbeimer $M D$, Katz AI.

The kidney and hypertension in pregnancy. In: Brenner BM, Rector FC Jr, eds. The Kidney. Philadelphia: Saunders, 1991b: 1551-1595.

Loeppky JA, Hoekenga DE, Greene ER, Luft UC.

Comparison of noninvasive Doppler and Fick measurements of stroke volume in cardiac patients. Am Heart J 1984;107:339-346.

Longo $L D$.

Maternal blood volume and cardiac output during pregnancy: a hypothesis of endocrinological control. Am J Physiol 1983;245:R720-R729.

Longo LD, Hardesty JS.

Maternal blood volume: measurement, hypothesis of control and clinical considerations. Rev Perinatal Med 1984;5:35-59.

Loue SA, MacDonald GJ, Brown MA.

Regulation of atrial natriuretic peptide release in pregnancy: responses to posture. Am J Obstet Gynecol 1991a;165:591-595.

Loue SA, Zammit VC, Mitar D, MacDonald GJ, Brown MA.

Atrial natriuretic peptide and plasma volume in pregnancy-induced hypertension. Am J Hypertens 1991b;4:897-903. 
Lowe SA, MacDonald GJ, Brown MA.

Atrial natriuretic peptide in pregnancy: response to oral sodium supplementation. Clin Exp Pharmacol Physiol 1992a;19:607-612.

Lowe SA, MacDonald GJ, Brown MA.

Acute and chronic regulation of atrial natriuretic peptide in human pregnancy: a longitudinal study. J Hypertens 1992b;10:821-829.

Lowe SA, Hetmanski DJ, MacDonald I, Brougbton Pipkin F, Rubin PC.

Intravenous volume expansion therapy in pregnancy-induced hypertension: the role of vasoactive hormones. Clin Exp Hypertens 1993;B12:139-151.

Lu ZM, Goldenberg RL, Cliver SP, Cutter G, Blankson M.

The relationship between maternal hematocrit and pregnancy outcome. Obstet Gynecol 1991;77:190-194.

Lund CJ, Donovan JC.

Blood volume during pregnancy. Significance of plasma and red cell volumes. Am J Obstet Gynecol 1967;98:393-403.

Lusiani L, Ronsisvalle G, Bonanome A, Visona A, Castellani V, Maccbia C, Pagnan A.

Echocardiographic evaluation of the dimensions and systolic properties of the left ventricle in freshman athletes during physical training. Eur Heart J 1986;7:196-203.

Mabie WC, Ratts TE, Sibai BM.

The central hemodynamics of severe preeclampsia. Am J Obstet Gynecol 1989;161:1443-1448.

Mabie WC, DiSessa TG, Crocker LG, Sibai BH, Arbeart KL.

A longitudinal study of cardiac output in normal human pregnancy. Am J Obstet Gynecol 1994;170:849-856.

MacGillivray 1, Rose GA, Rowe B.

Blood pressure survey in pregnancy. Clin Sci 1969;37:395-407.

MacGillivray I, Campbell D, Duffus GM.

Maternal metabolic response to twin pregnancy in primigravidae. J Obstet Gynaecol Br Commonw 1971;78:530-534.

Manasc B, Jepson J.

Erythropoietin in plasma and urine during human pregnancy. Can Med Assoc J 1969;100:687-691.

Marx GF, Schwalbe SS, Cbo E, Whitty JE.

Automated blood pressure measurements in laboring women: are they reliable? Am J Obstet Gynecol 1993;168: 796-798.

Masaki DI, Greenspoon JS, Ouzounian JG.

Measurement of cardiac output by thoracic electrical bioimpedance and thermodilution. Am J Obstet Gynecol 1989;161:680-684.

Masbini IS, Albazzaz SJ, Fadel HE, Abdulla AM, Hadi HA, Harp R, Devoe LD.

Serial noninvasive evaluation of cardiovascular hemodynamics during pregnancy. Am J Obstet Gynecol 1987;156:1208-1213.

Mattbeus JNS, Altman DG, Campbell MJ, Royston P.

Analysis of serial measurements in medical research. Br Med J 1990;300:230-235.

McCance DR, McKnight JA, Traub AI, Sberidan B, Roberts G, Atkinson AB.

Plasma atrial natriuretic factor levels during normal pregnancy and pregnancy complicated by diabetes mellitus and hypertension. J Hum Hypertens 1990;4:31-35.

McLennan CE, Louenstein JM.

Blood volume changes immediately after delivery. Stanford M Bull 1959;17:152-156.

McLennan FM, Haites NE, Rawles JM.

Stroke and minute distance in pregnancy: a longitudinal study using Doppler ultrasound. Br J Obstet Gynaecol 1987;94:499-506.

Milsom I, Forssman L, Sivertsson R, Dottori $O$.

Measurement of cardiac stroke volume by impedance cardiography in the last trimester of pregnancy. Acta Obstet Gynecol Scand 1983;62:473-479.

Milsom I, Forssman L.

Factors influencing aortocaval compression in late pregnancy. Am J Obstet Gynecol 1984;148:764-771. 
Milsom I, Hedner J, Hedner T.

Plasma atrial natriuretic peptide (ANP) and maternal hemodynamic changes during normal pregnancy. Acta Obstet Gynecol Scand 1988;67:717-722.

Mintz GS, Kotler MN, Parry WR, Iskandrian AS, Kane SA.

Real-time inferior vena caval ultrasonography: normal and abnormal findings and its use in assessing right-heart function. Circulation 1984;64:1018-1024.

Moe GW, Legault L, Skorecki KL.

Disturbances in control of body fluid volume and composition. In: Brenner BM, Rector FC Jr, eds. The Kidney. Philadelphia: Saunders, 1991: 623-677.

Moreno AH, Katz AI, Gold LD, Reddy RV.

Mechanics of distension of dog veins and other very thin-walled tubular structures. Circ Res 1973;27:1069-1080.

Moreno FL, Hagan AD, Holmen JR, Pryor TA, Strickland RD, Castle CH.

Evaluation of size and dynamics of the inferior vena cava as an index of right-sided cardiac function. Am J Cardiol 1984;53:579-585.

Moutquin JM, Rainville C, Ginoux L, Raynauld P, Amyot G, Bilodeau R, Pelland N.

A prospective study of blood pressure in pregnancy: prediction of preeclampsia. Am J Obstet Gynecol 1985;151: 191-196.

Mulay $S$, D'Sylva $S$, Varma DR.

Inhibition of the aldosterone-suppressant activity of atrial natriuretic factor by progesterone and pregnancy in rats. Life Sci 1993;52:1121-1128.

Murpby JF, O'Riordan J, Newcombe RG, Coles EC, Pearson JF.

Relation of haemoglobin levels in first and second trimesters to outcome of pregnancy. Lancet 1986;i:992-995.

Nakao S, Come PC, McKay RG, Ransil BJ.

Effects of positional changes on inferior vena caval size and dynamics and correlations with right-sided cardiac pressure. Am J Cardiol 1987;59:125-132.

Natori H, Tamaki S, Kira S.

Ultrasonographic evaluation of ventilatory effect on inferior vena caval configuration. Am Rev Resp Dis 1979;120: 421-427.

\section{Newman B}

Cardiac output changes during caesarean section: measurements by transcutaneous aortovelography. Anaesthesia $1982 ; 37: 270-273$.

Nisell $H$, Lunell $N$, Linde $B$.

Maternal hemodynamics and impaired fetal growth in pregnancy-induced hypertension. Obstet Gynecol 1988;71: 163-166.

Nisell H, Carlstrom K, Cizinsky S, Grunewald C, Nylund L, Randmaa I.

Atrial natriuretic peptide concentrations and hemodynamic effects of acute plasma volume expansion in normal pregnancy and preeclampsia. Obstet Gynecol 1992;79:902-907.

Nixon JV, Murray RG, Leonard PD, Mitcbell JH, Blomqvist CG.

Effect of large variations in preload on left ventricular performance characteristics in normal subjects. Circulation 1982;65:698-703.

Nolten WE, Lindbeimer MD, Oparil S, Rueckert PA, Ebrlich EN.

Desoxycorticosterone in normal pregnancy. II. Cortisol-dependent fluctuations in free plasma desoxycorticosterone. Am J Obstet Gynecol 1979;133:644-648.

Oats JN, Brougbton Pipkin F, Symonds EM, Craven DJ.

A prospective study of plasma angiotensin converting enzyme in normotensive primigravidae and their infants.

Br J Obstet Gynaecol 1981;88:1204-1210.

Oparil S, Ebrlich EN, Lindheimer MD.

Effect of progesterone on renal sodium handling in man: relation to aldosterone secretion and plasma renin activity. Clin Sci Mol Med 1975;49:139-147.

Ouens JA, Falconer J, Robinson JS.

Effect of restriction of placental growth on umbilical and uterine blood flows. Am J Physiol 1986;250:R427-R434. 
Page EW, Christianson $R$.

The impact of mean arterial blood pressure to toxemia of pregnancy in the primigravid patient. Am J Obstet Gynecol $1976 ; 125: 740-746$.

\section{Palmer AJ, Walker AHC.}

The maternal circulation in normal pregnancy. J Obstet Gynaecol Br Empire 1949;56:537-547.

\section{Patterson RM, Gibbs CE, Wood RC.}

Birthweight percentile and perinatal outcome: Recurrence of intrauterine growth retardation. Obstet Gynecol $1986 ; 68: 464-468$.

\section{Pedersen EB, Jobannesen $P$, Rasmussen AB, Danielsen $H$.}

The osmoregulatory system and the renin-angiotensin-aldosterone system in preeclampsia and normotensive pregnancy. Scand J Clin Lab Invest 1985;45:627-633.

\section{Peeters LLH, Verkeste CM, Saxena PR, Wallenburg HCS.}

Relationship between maternal hemodynamics and hematocrit and hemodynamic effects of isovolemic hemodilution and hemoconcentration in the awake late-pregnant guinea pig. Ped Res 1987;21:584-589.

\section{Peeters LLH, Buchan PC.}

Blood viscosity in perinatology. Rev Perinatal Med 1989;6:53-89.

Pbelan JP, Yurtb DA.

Severe preeclampsia: I. Peripartum hemodynamic observations. Am J Obstet Gynecol 1982;144:17-22.

\section{Pbippand AF, Horvath JS, Glynn EM, Garner MG, Fletcber PJ, Duggin GG, Tiller DJ.}

Circulatory adaptation to pregnancy - serial studies of haemodynamics, blood volume, renine and aldosterone in the Baboon (Papio hamadryas). J Hypertens 1986;4:773-779.

Pbippard AF, Hodsman GP, Horvath JS, Jobnston CI, Duggin GG, Tiller DJ.

Sequential studies on atrial natriuretic peptide in pregnant and non-pregnant baboons. Kidney Int 1988;33:140 (abstract).

Pijnenbong R, Antbony J, Davey DA, Rees A, Tiltman A, Vercruysse L, Van Asscbe A.

Placental bed spiral arteries in the hypertensive disorders of pregnancy. Br J Obstet Gynaecol 1991;98:648-655.

Pirani BBK, Campbell DM, MacGillivray I.

Plasma volume in normal first pregnancy. J Obstet Gynaecol Br Commonw 1973;80:884-887.

Pirani BBK, MacGillivray I.

Smoking during pregnancy. Its effect on maternal metabolism and fetoplacental function. Obstet Gynecol $1978 ; 52: 257-263$.

Pocock SJ.

Clinical trials: a practical approach. Chichester: Wiley, 1983.

Poulsen $\mathrm{H}$, Stjernquist $M$, Sjöbeng $\mathrm{N}-\mathrm{O}$.

Is ANP responsible for the hemoconcentration in preeclampsia? Med Hypotheses 1993;41:235-238.

Pratber JW, Taylor AE, Guyton AC.

Effect of blood volume, mean circulatory pressure, and stress relaxation on cardiac output. Am J Physiol $1969 ; 216: 467-472$.

Pritchard JA, Barnes AC, Brigbt RH.

The effect of supine posture on renal function in near-term pregnant women. J Clin Invest 1955;34:777-781.

Pritchard JA.

Blood volume changes in pregnancy and the puerperium. IV. Anemia associated with hydatiform mole. Am J Obstet Gynecol 1964;91:621-629.

Pritcbard JA.

Changes in the blood volume during pregnancy and delivery. Anesthesiology 1965;26:393-399.

Quilligan EJ, Tyler C.

Postural effects on the cardiovascular status in pregnancy: a comparison of the lateral and supine postures. Am J Obstet Gynecol 1959;78:465-471.

Rafferty TD, Berkowitz RL.

Hemodynamics in patients with severe toxemia during labor and delivery. Am J Obstet Gynecol 1980;138:263-270. 
Raiba CE.

Prematurity, perinatal mortality and maternal heart volume. Guy's Hosp Rep 1964;113:96-110.

Rak KM, Hopper KD, Tyler HN.

The slit infrahepatic IVC: pathologic entity or normal variant? J Clin Ultrasound 1991;19:399-403.

Ramsay B, De Belder A, Campbell S, Moncada S, Martin JF.

A nitric oxide donor improves uterine artery diastolic blood flow in normal early pregnancy and in women at high risk of pre-eclampsia. Eur J Clin Invest 1994;24:76-78.

Rawles JM, Scbneider KTM, Hucb R, Hucb A.

The effect of position and delay on stroke and minute distance in late pregnancy. Br J Obstet Gynaecol 1987;94:507511.

Reckelboff JF, Samsell L, Baylis C.

Failure of an acute $10-15 \%$ plasma volume expansion in the virgin female rat to mimic the increased glomerular filtration rate and altered glomerular hemodynamics seen at midterm pregnancy. Clin Exp Hypertens 1989;B8:533-549.

Rector WG, Adair O, Hossack KF, Rainguet S.

Atrial volume in cirrhosis: relationship to blood volume and plasma concentration of atrial natriuretic factor. Gastroenterology 1990;99:766-770.

Reiss $R$, O'Sbaugbnessy R, Quilligan T, Zuspan F.

Retrospective comparison of blood pressure course during preeclamptic and matched control pregnancies. Am J Obstet Gynecol 1985;156:894-888.

Roberts JM, Taylor RN, Musci TJ, Rodgers GM, Hubel CA, McLaugblin MK.

Preeclampsia, an endothelial cell disorder. Am J Obstet Gynecol 1989;161:1200-1204.

Roberts JM, Taylor RM, Goldfein A.

Clinical and biochemical evidence of endothelial cell dysfunction in the pregnancy syndrome preeclampsia. Am J Hypertens 1991;4:700-708.

Robinson BF, Epstein SE, Kabler RL, Braunwald E.

Circulatory effects of acute expansion of blood volume: studies during maximal exercise and at rest. Circ Res $1966 ; 19: 26-32$.

Robson SC, Dunlop W, Boys RJ, Hunter S.

Cardiac output during labour. Br Med J 1987a;295:1169-1172.

Robson SC, Hunter S, Moore M, Dunlop W.

Haemodynamic changes during the puerperium: a Doppler and M-mode echocardiographic study. Br J Obstet Gynaecol 1987b;94:1028-1039.

Robson SC, Hunter S, Dunlop W.

Left atrial dimensions during early puerperium. Lancet 1987c;ii:111-112.

Robson SC, Dunlop W, Moore M, Hunter S.

Combined Doppler and echocardiographic measurement of cardiac output: theory and application in pregnancy.

Br J Obstet Gynaecol 1987d;94:1014-1027.

Robson SC, Hunter S, Boys RJ, Dunlop W.

Serial study of factors influencing changes in cardiac output during human pregnancy. Am J Physiol 1989a;256:H1060-H1065.

Robson SC, Hunter S, Boys RJ, Dunlop W.

Hemodynamic changes during twin pregnancy. A Doppler and M-mode echocardiographic study. Am J Obstet Gynecol 1989b;161:1273-1278.

Robson SC, Boys RJ, Hunter S, Dunlop W.

Maternal hemodynamics after normal delivery and delivery complicated by postpartum hemorrhage. Obstet Gynecol $1989 \mathrm{c} ; 74: 234-239$.

Robson SC, Hunter S, Boys RJ, Dunlop W.

Serial changes in pulmonary haemodynamics during human pregnancy: a non-invasive study using Doppler echocardiography. Clin Sci 1990;80:113-117. 
Robson SC, Dunlop W.

Letter to the editor: When do cardiovascular parameters return to their preconception values? Am J Obstet Gynecol 1992:167:1479.

Rogers PA, Polson D, Murpby CR, Hosie M, Susil B, Leoni M.

Correlation of endometrial histology, morphometry, and ultrasound appearance after different stimulation protocols for in vitro fertilization. Fertil Steril 1991;55:583-587.

Rasso P, Donoso E, Braun S, Espinoza $R$, Fernandez C, Salas SP.

Maternal hemodynamic adjustments in idiopathic fetal growth retardation. Gynecol Obstet Invest 1993;35:162-165.

Rotbe $C F$.

Reflex control of veins and vascular capacitance. Physiol Rev 1983;63:1281-1339.

Rotbe CF.

Physiology of venous return. Arch Intern Med 1986;146:977-982.

Rovinsky. IJ, Jaffin $\mathrm{H}$.

Cardiovascular hemodynamics in pregnancy. I. Blood and plasma volumes in multiple pregnancy. Am J Obstet Gynecol 1965;93:1-13.

Rovinsky II, Jaffin $H$.

Cardiovascular hemodynamics in pregnancy. II. Cardiac output and left ventricular work in multiple pregnancies. Am J Obstet Gynecol 1966;95:781-786.

Rubler S, Scbneebaum R, Hammer $N$.

Systolic time intervals in pregnancy and the post-partum period. Am Heart J 1973;86:182-188.

Rubler S, Damani PM, Pinto ER.

Cardiac size and performance during pregnancy estimated with echocardiography. Am J Cardiol 1977;40:534-540.

Rutberford AJ, Anderson JV, Elder MG, Bloom SR.

Release of atrial natriuretic peptide during pregnancy and immediate puerperium. Lancet 1987;i:928-929.

Sadaniantz A, Kocberil AG, Emaus SP, Garber CE, Parisi AF.

Cardiovascular changes in valuated by two-dimensional and Doppler echocardiography. Am J Soc Echocardiogr 1992;5:253-258.

Sady MA, Haydon BB, Sady SP, Carpenter MW, Thompson PD, Coustan DR.

Cardiovascular response to maximal cycle exercise during pregnancy and at two and seven months post partum. Am J Obstet Gynecol 1990;162:1181-1185.

Sabn DJ, De Maria A, Kisslo J, Weyman A.

Recommendations regarding quantifications in M-mode echocardiographic measurements. Circulation 1978;58: 1072-1083.

Salas $S P$, Rosso P, Espinoza $R$, Robert J, Valdés $G$, Donoso E.

Maternal plasma volume expansion and hormonal changes in women with idiopathic fetal growth retardation. Obstet Gynecol 1993;81:1029-1033.

Sarrel PM, Lindsay DC, Poole-Wilson PA, Collins P.

Hypothesis: inhibition of endothelium-derived relaxing factor by haemoglobin in the pathogenesis of pre-eclampsia. Lancet 1990;336:1030-1032.

Schrier RW, Dürr JA.

Pregnancy: an overfill or underfill state. Am J Kidney Dis 1987;9:284-289.

Scbrier RW, Briner VA.

Peripheral arterial vasodilatation hypothesis of sodium and water retention in pregnancy: Implications for pathogenesis of preeclampsia-eclampsia. Obstet Gynecol 1991;77:632-639.

Scbuster AH, Nanda NC.

Doppler echocardiographic measurement of cardiac output: comparison with a non-golden standard. Circulation 1984;53:257-259.

Sealey JE, Cbolst I, Glorioso N, Troffa C, Weintraub ID, James G, Laragb JH.

Sequential changes in plasma LH and plasma prorenin during the menstrual cycle. J Clin Endocrinol Metab 1987;63:1-5. 
Seeley EW, Williams GH, Graves SW.

Markers of sodium and volume homeostasis in pregnancy-induced hypertension. J Clin Endocrinol Metab 1992;74:150-156.

Sbennan AH, Kissane J, De Swiet $M$.

Validation of the SpaceLabs 90207 ambulatory blood pressure monitor for use in pregnancy. Br J Obstet Gynaecol 1993; 100:904-908.

Sibai BM, Anderson GD, Spinnato JA, Sbaver DC.

Plasma volume findings in patients with mild pregnancy-induced hypertension. Am J Obstet Gynecol 1983;147:16-19.

Siimes ASL, Immonen I, Stenman U, Kärkkäinen JEK, Pekonen F, Fybrquist FY.

Plasma renin substrate in the prediction of pregnancy outcome in threatened abortion. $\mathrm{Br} \mathrm{J}$ Obstet Gynaecol 1983;90:1186-1192.

\section{Simonson JS, Scbiller NB.}

Sonospirometry: a new method for noninvasive estimation of mean right atrial pressure based on two-dimensional echographic measurements of the inferior vena cava during measured inspiration. J Am Coll Cardiol 1988;11:557-564.

Sims EA, Krantz KE.

Serial studies of renal function during pregnancy and the puerperium in normal women. J Clin Invest 1958;37:17641774.

Skinner SL, Lumbers ER, Symonds EM.

Analysis of changes in the renin-angiotensin system during pregnancy. Clin Sci 1972;42:479-488.

Slater AJ, Gude N, Clarke IJ, Walter WA.

Haemodynamic changes and left ventricular performance during high dose oestrogen administration to male transsexuals. Br J Obstet Gynaecol 1986;93:532-538.

Smeaton TC, Andersen GJ, Fulton IS.

Study of aldosterone levels in plasma during pregnancy. J Clin Endocrinol Metab 1977;44:1-7.

Stander HJ, Cadden JF.

Cardiac output in pregnant women. Am J Obstet Gynecol 1932;24:13-19.

Steegers EAP, Hein PR, Tan ACIT, Groeneveld EAM, Jongsma HW, Benraad TJ.

Plasma atrial natriuretic peptide (ANP) in late pregnancy and puerperium. Eur J Obstet Gynecol Reprod Biol 1987;26:213-217.

Steegers EAP.

Salt and pregnancy [Dissertation]. Nijmegen: Katholieke Universiteit Nijmegen, 1990.

Steegers EAP, Steegers-Tbeunissen RPM, Jongsma HW, Hein PR.

Atrial natriuretic peptide in the first gestational trimester: a longitudinal study. Gynecol Obstet Invest 1991;31:246-248.

Stevenson JC, MacDonald DWR, Warren RC, Booker MW, Wbitebead MJ.

Increased concentration of circulating calcitonin gene-related peptide during normal human pregnancy. $\mathrm{Br} \mathrm{Med} \mathrm{J}$ 1986;294:1329-1330.

Stewart WJ, Jiang L, Micb R, Pandian S, Guerrero JL, Weyman AE.

Variable effects of changes in flow rate through the aortic, pulmonary and mitral valves on valve area and flow velocity: Impact on quantitative Doppler flow calculations. J Am Coll Cardiol 1985;6:653-662.

Stratta P, Canavese C, Gurioli L, Todros T, Fiancbino O, Brosso PC, Censon A, Pluviano F.

Atrial natriuretic factor as a marker of the intravascular volume conditions in normal and hypertensive pregnancies.

Clin Exp Hypertens 1988;B7:107-111.

Stratta P, Canavese C, Dogliani M, Gurioli L, Porcu MC, Todros T, Fianchino O, Benedetto C, Massobrio M, Balbi L. Repeated albumin infusions do not lower blood pressure in preeclampsia. Clin Nephrol 1991;36:234-239.

Strauss RG, Keefer JR, Burke T, Civetta JM.

Hemodynamic monitoring of cardiogenic pulmonary edema complicating toxemia of pregnancy. Obstet Gynecol $1980 ; 55: 170-174$. 
Symonds EM, Brougbton Pipkin F.

Pregnancy hypertension, parity and the renin-angiotensin system. Am J Obstet Gynecol 1978;132:473-479.

Symonds EM, Brougbton Pipkin F.

Factors affecting plasma angiotensin II concentration in labour. Br J Obstet Gynaecol 1980;87:869-874.

Taylor DJ, Lind T.

Puerperial haemotological indices. Br J Obstet Gynaecol 1981;88:601-606.

Taylor RN, Heilbron DC, Roberts JM.

Growth factor activity in the blood of women in whom preeclampsia develops is elevated from early pregnancy. Am J Obstet Gynecol 1990;163:1839-1844.

Thompson JA, Hays PM, Sagar KB, Cruiksbank DP.

Echocardiographic left ventricular mass to differentiate chronic hypertension from preeclampsia during pregnancy. Am J Obstet Gynecol 1986;155:994-999.

Thomsen JK, Storm TL, Thamsbong G, De Nully M, Bodker B, Skouby S.

Atrial natriuretic peptide concentrations in pre-eclampsia. Br Med J 1987;294:1508-1510.

Tbomsen JK, Storm TL, Thamsborg G, de Nully M, Bodker B, Skouby SO.

Increased concentration of circulating atrial natriuretic peptide during normal pregnancy. Eur J Obstet Gynecol Reprod Biol 1988;27:197-201.

\section{Thomson JK, Fogb-Andersen N, Bulow K, Devantier A.}

Blood and plasma volumes determined by carbonmonoxide gas, ${ }^{9 m-T}$ T labelled erythrocytes, ${ }^{18} \mathrm{I}$-albumin and the T1824 technique. Scand J Clin Lab Invest 1991;51:185-190.

Tbomsen JK, Fogb-Andersen N, Jaszczak P, Giese J.

Atrial natriuretic peptide decrease during normal pregnancy as related to hemodynamic changes and volume regulation. Acta Obstet Gynecol Scand 1993;72:103-110.

Thomsen JK, Fogb-Andersen N, Jaszcazk P.

Atrial natriuretic peptide, blood volume, aldosterone, and sodium excretion during twin pregnancy. Acta Obstet Gynecol Scand 1994;73:14-20.

Turner $A F$.

The chest radiograph during pregnancy. Clin Obstet Gynecol 1975;18:65-74.

Veland $K$, Hansen JM.

Maternal cardiovascular dynamics. II. Posture and uterine contractions. Am J Obstet Gynecol 1969a;103:1-7.

Ueland $K$, Hansen JM.

Maternal cardiovascular dynamics. III. Labor and delivery under local and caudal analgesia. Am J Obstet Gynecol 1969b;103:8-18.

\section{Ueland K, Novy MJ, Peterson EN, Metcalfe J.}

Maternal cardiovascular dynamics. IV. The influence of gestational age on the maternal cardiovascular response to posture and exercise. Am J Obstet Gynecol 1969c;104:856-864.

\section{Ueland $K$.}

Maternal cardiovascular dynamics. VII. Intrapartum blood volume changes. Am J Obstet Gynecol 1976;126:671-677.

\section{Unnerus CE.}

Heart volume and prematurity. Acta Obstet Gynecol Scand 1959;38:340-351.

Valenzuela GF, Kim S, Rauld HF.

Cardiovascular changes after closure of uterine circulation during pregnancy. Am J Physiol 1990;258 (Regulatory Integrative Comp Physiol 27):R1431-1435.

Van den Broek W, Trouwborst A, Bakker WH.

The effect of iso-oncotic plasma substitutes: gelatine, dextran 40 and the effect of Ringer's lactate on the plasma volume in healthy subjects. Acta Anaesthesiol Belg 1989;40:275-280.

Veille JC, Morton MJ, Burry KJ.

Maternal cardiovascular adaptation to twin pregnancy. Am J Obstet Gynecol 1985;153:261-263.

Veille JC, Morton MJ, Burry K, Nemetb M, Speroff L.

Estradiol and hemodynamics during ovulation induction. J Clin Endocrinol Metab 1986;63:721-724. 
Veille JC, Morton MJ, Paul MS.

Matemal left ventricular dimension in pregnancies complicated by fetal growth retardation. Obstet Gynecol 1991;78:265-269.

Vered Z, Poler SM, Gibson P, Wlody D, Perez JE.

Noninvasive detection of the morphologic and hemodynamic changes during normal pregnancy. Clin Cardiol 1991;14:327-334.

Verel D, Bury JD, Hope A.

Blood volume changes in pregnancy and the puerperium. Clin Sci 1956;15:1-7.

Villar J, Repke J, Markusb L, Calvert W, Rboads G.

The measuring of blood pressure during pregnancy. Am J Obstet Gynecol 1989;161:1019-1024.

Vinatier D, Tiffet $O$, Dufour P, Tibergbeim B, Maunoury-Lefebre C, Monnier JC.

Cytokines et grossesse: physiologie. J Gynecol Obstet Biol Reprod (Paris) 1992;21:535-543.

Visser W, Van den Dorpel MA, Derkx FHM, Wallenbung HCS, Schalekamp MADH.

Atrial natriuretic peptide and haemodynamics in untreated pre-eclampsia. J Hypertens 1987;5:S33-S35.

Visser W, Wallenburg HCS.

Central hemodynamic observations in untreated preeclamptic patients. Hypertension 1991;17:1072-1077.

Vokes T7, Weiss NM, Scbreiber J, Gaskill MB, Robertson GL.

Osmoregulation of thirst and vasopressin during normal menstrual cycle. Am J Physiol 1988;254:R641-R647.

Voorborst FJ, Puyenbroek JI, Robertson EA, Bezemer PD, Kurver PHJ.

Verschillen de geboortegewichten van vroeger en nu? (Differences between earlier and current birth weights?)

Ned Tijdschr Geneeskd 1990:134:998-1002.

Vorys $N$, Ullery JC, Hanusek GE.

The cardiac output changes in various positions in pregnancy. Am J Obstet Gynecol 1961;82:1312-1321.

Vytiska-Binstorfer E, Huber JC, Havel $M$.

ANP-, Aldosteron und ADH-verlauf während der normalen Schwangerschaft. Geburtshilfe Frauenheilkd $1989 \mathrm{a} ; 49: 902-905$.

Vytiska-Binstorfer E, Huber J, Fitz $R$, Hartter E, Hawel $M$.

Das atriale natriuretische Peptid (ANP), das antidiuretische Hormon (ADH) und Aldosteron während Geburt und

Wochenbett. Z Geburtshilfe Perinatol 1989b;193:211-214.

Wallenburg HCS.

Hemodynamics in hypertensive pregnancy. In: Rubin PC, ed. Hypertension in pregnancy. Amsterdam: Elsevier Science Publishers, 1988: 66-101.

Walters WAW, MacGregor WG, Hills $M$.

Cardiac output at rest during pregnancy and the puerperium. Clin Sci 1966;30:1-11.

Walters WAW, Lim YL.

Blood volume and haemodynamics in pregnancy. Clin Obstet Gynaecol 1975;2:301-313.

Wasserstrum N, Kirshon B, Willis RS, Moise KJ, Cotton DB.

Quantitative hemodynamic effects of acute volume expansion in severe preeclampsia. Obstet Gynecol 1989;73:

546-550.

Wasserstrum $N$.

Issues in fluid management during labor: maternal plasma volume status and volume loading. Clin Obstet Gynecol 1992;35:514-526.

Weir RJ, Paintin DB, Brown J, Fraser R, Lever AF, Robertson JIS, Young J.

A serial study in pregnancy of the plasma concentrations of renin, corticosteroids, electrolytes and proteins and of haematocrit and plasma volume. J Obstet Gynaecol Br Commonw 1971;78:590-602.

Weir RJ, Fraser R, Lever AF, Morton IJ, Brown IJ, Kraszewski A, McIlwaine GM, Robertson JIS, Tree M.

Plasma renin, renin substrate, angiotensin II and aldosterone in hypertensive disease of pregnancy. Lancet 1973; i: 291-294. 
Weir RJ, Brown J, Fraser R, Lever AF, Logan RW, McIlwaine GM, Morton J, Robertson JIS, Tree M.

Relationship between plasma renin, renin-substrate, angiotensin II, aldosterone and electrolytes in normal pregnancy. J Clin Endocrinol Metab 1975;40:108-115.

Welcker $H$.

Blutkörperzählung und farbeprüfende Methode. Prag Vierteljahrschr prakt Heilk 1854;4:11-80.

Whittaker $P G$, Lind T.

The intravascular mass of albumin during human pregnancy: a serial study in normal and diabetic women. Br J Obstet Gynaecol 1993;100:587-592.

Wicbman K, Ryden G, Wicbman G.

The influence of different positions and Korotkoff sounds on the blood pressure measurements in pregnancy. Acta Obstet Gynecol Scand (Suppl) 1984;118:25-28.

\section{Wícbman $K$, Rydén $G$.}

Blood pressure and renal function during normal pregnancy. Acta Obstet Gynecol Scand 1986;65:561-566,

Williams MA, Goldman MB, Mittendorf R, Monson RR.

Subfertility and the risk of low birth weight. Fertil Steril 1991;56:668-671.

Winner W, Romney SL.

Cardiovascular responses to labor and delivery. Am J Obstet Gynecol 1966;95:1104-1114. 


\section{DANKWOORD}

De studies, die worden beschreven in dit proefschrift, werden verricht op de afdelingen gynaecologie, cardiologie, klinische chemie en nucleaire geneeskunde van het Academisch Ziekenhuis te Maastricht. Het proefschrift beschrijft bevindingen bij proefpersonen en zwangere patiënten, zonder wiens welwillende en gemotiveerde medewerking dit onderzoek niet mogelijk was geweest. Door de uitgave van deze dissertatie is een volgende stap gezet in de ontrafeling van de hemodynamische fysiologie van de zwangerschap. Mijn dank aan u allen.

Een persoonlijk woord van dank richt ik tot de volgende personen:

- Zeergeleerde Peeters, Louis, zonder jou was er nooit wat van terecht gekomen. Jouw ontembare enthousiasme bracht mij vaak tot wanhoop, maar even zo vaak wist je me te enthousiasmeren over weer een nieuwe kijk op de resultaten. De vele bezoeken aan Maastricht ( 25 duizend kilometers in 2 jaar) waren de afgelopen jaren een wetenschappelijke Erholung. Maar niet alleen de wetenschap, maar ook zakelijke en persoonlijke aangelegenheden passeerden tijdens onze sessies de revue.... "Louis, this is the beginning of a beautiful friendship" (Humphrey Bogart in de slotscène van Casablanca, 1942).

- Hooggeleerde de Haan, professor, één ding kan ik u verzekeren: ik vergeet u nooit. Dank voor uw opleiding, met name voor uw oneindig streven ons wetenschappelijk te motiveren, en zoals u ziet ... ook nu is het weer één van de Jeltjes gelukt om een vriendje te worden.

- Geleerde Cheriex, Emile, zonder jouw exacte echocardiografische bepalingen was mijn onderzoek niet mogelijk geweest. Geen (Doppler) golf was jou ooit te hoog om met raad en daad in het onderzoek te participeren. Veel dank en waardering hiervoor.

- Geleerde Pieters, Frans, dank voor je inzet, met name voor je persoonlijke betrokkenheid en het vaak even tussendoor verrichten van een echocardiografie.

- Zeergeleerde Menheere, Paul, dankzij jouw hulp werd het klinisch-chemisch deel van dit onderzoek nauwkeurig afgerond.

- Zeergeleerde Heidendal, dank voor uw belangstelling en medewerking bij het verrichten van de volume belastings experimenten.

- Geleerde Tan, Wie Djin, bedankt voor je praktische hulp bij het onderzoek en voor het kritisch doorlezen van de manuscripten.

- Hooggeleerde leden van de beoordelingscommissie, u allen dank ik voor de toetsing van het manuscript.

- Professor Davison, your work in the 80 s inspired us to start this research. I am very honoured because of your interest in this thesis and your presence at the graduation ceremony.

- Sehr geehrter Nyfeler, Fritz, vielen Dank für die Ubersetzung der Zusammenfassung in meine Muttersprache, das Bärndütsch. 
- Medewerksters van de hartfunctie afdeling, bedankt voor jullie hulp bij de metingen en het organiseren van de afspraken.

- Medewerkers van het klinisch chemisch laboratorium en het laboratorium voor immunochemie, bedankt voor het afdraaien en verwerken van de honderden bloed- en urine monsters. Speciaal dank aan Riet Schmitz en José VrankenVranken voor het verrichten van de bepalingen, die vaak nog liever gisteren dan vandaag klaar moesten.

- Medewerkers van de afdeling nucleaire geneeskunde, Servé Halders en Sandra Limny verrichtten nauwkeurig de plasma volume bepalingen.

- (Zeer)geleerde maatschapsleden gynaecologie van het St. Clara Ziekenhuis, bedankt voor jullie geduld en de mogelijkheden om dit werk af te ronden.

- Verpleegkundigen van de afdelingen gynaecologie, verloskunde, verloskamers en polikliniek gynaecologie van het AZM, bedankt voor alles.

- Medewerkers van de bibliotheek van het AZM, De Wever-Ziekenhuis en het St. Clara Ziekenhuis, bedankt voor het bijeenzoeken van de meer dan 400 referenties.

- Zeergeleerde Schouten, met dank voor de reddingsoperatie van onze statistische bewerkingen.

- Zeergeleerde Stewart, Patricia, zeergeleerde Grootendorst, Albert, dank voor jullie meedenken en advies.

- Geachte van Rossum, posthuum komt u de eer toe aan de basis van dit proefschrift te staan. Als schooldecaan wist $\mathrm{u}$ te bewerkstelligen dat ik voor exact koos en dat dit geen proefschrift klassieke talen is geworden.

Liefste Hélène, samen met Martijne en Carlijn zijn jullie misschien wel degenen, die het meest hebben moeten afzien tijdens de fabricage van dit proefschrift. Het oogst veel bewondering hoe je ons gezin intussen hebt gerund. Dankzij jouw ondersteuning en aansporingen is dit werk dan toch afgekomen. 
PUBLICATIONS 


\section{PAPERS}

\section{Duvekot JJ, van Muyden RCPM.}

Inhibition of spermatogenesis (letter to the editor). Fertil Steril 1986;46:341.

Duvekot JJ, Alberda ATh, Drogendijk AC.

A comparison of the penetration of human sperm into bovine and artificial mucus (letter to the editor). Fertil Steril 1987; $47: 363$.

Duvekot JJ, Theewes BAM, Wesdorp JM, Roumen FJME, Bouckaert PXJM.

Congenital cytomegalovirusinfection in a twin pregnancy: a case report. Eur J Pediat 1990;149:261-262.

Duvekot JJ, Cheriex EC, Pieters FAA, Menbeere PPCA, Peeters LLH.

Early pregnancy changes in hemodynamics and volume homeostasis are consecutive adjustments triggered by a primary fall in systemic vascular tone. Am J Obstet Gynecol 1993;169:1382-1392.

Duvekot JJ, Cheriex EC, Tan WD, Heidendal GAK, Peeters LLH.

Volume-dependent echocardiographic parameters are not useful for estimating baseline blood volume but are useful for detecting acute changes in vascular filling state. Basic Res Cardiol 1994;89:270-277.

Duvekot JJ, Cheriex EC, Tan WD, Heidendal GAK, Peeters LLH.

Measurement of anterior-posterior diameter of inferior vena cava by ultrasonography: a new noninvasive method to assess acute changes in vascular filling state. Cardiovasc Res 1994;28:1269-1272.

\section{Duvekot JJ, Peeters LLH.}

Maternal cardiovascular hemodynamic adaptation to pregnancy. Obstet Gynecol Surv 1994; in press.

\section{Duvekot JJ, Peeters LLH.}

Renal hemodynamics and volume homeostasis in pregnancy. Obstet Gynecol Surv 1994; in press.

Peeters LLH, Duvekot JJ.

The very early changes in maternal cardiovascular physiology. In: Clinical physiology in obstetrics. Chamberlain G, Broughton Pipkin F eds. Oxford: Blackwell Scientific Publication 1995, in press.

Duvekot JJ, Cheriex EC, Pieters FAA, Menheere PPCA, Peeters LLH.

Maternal volume homeostasis in early pregnancy in relation to fetal growth restriction. Obstetrics \& Gynecology 1995; in press.

Duvekot JJ, Cheriex EC, Pieters FAA, Menheere PPCA, Peeters LLH.

Severely impaired fetal growth is preceded by maternal hemodynamic maladaptation in very early pregnancy. Submitted for publication.

Van Oppen ACC, Van der Tweel I, Duvekot JJ, Bruinse HW.

Use of cardiac index in pregnancy: is it justified? Submitted for publication. 


\section{ABSTRACTS AND PRESENTATIONS}

\section{Duvekot JJ, Evers JLH, van Hemel OJS, de Haan J.}

The fetal duplication sign, an antenatal clue to triploidy? In: Abstracts 12th World Congress of Gynecology and Obstetrics, Rio de Janeiro, Brasil, 1988.

Duvekot JJ, Evers JLH, Cheriex EC, Pieters FAA, Menheere PPCA, Peeters LLH. Hemodynamic and renal changes in early pregnancy are influenced by embryoendometrial interaction. In: Abstracts 7th Reinier de Graaf Symposium, Maastricht, 1990.

\section{Duvekot JJ, Cheriex EC, Pieters FAA, Menheere PPCA, Peeters LLH.}

Vroege adaptatie van de circulatie in de zwangerschap bij de mens. Mini-symposium moederlijke herkennning van en adaptatie aan de zwangerschap, Nijmegen, 1990.

Duvekot JJ, Hasaart THM, Alleman JSP.

Een zeldzame complicatie bij pre-eclampsie. Wetenschappelijke Vergadering Nederlandse Vereniging voor Obstetrie en Gynaecologie, Rotterdam, 1990. Ned Tijdschr Geneeskd 1991;135:348.

Duvekot JJ, Evers JLH, Cheriex EC, Pieters FAA, Menheere PPCA, Peeters LLH.

Altered early hemodynamic-renal adaptation to pregnancy: a potential contributor to the later development of IUGR and/or preeclampsia. 7th International Congress of the ISSHP, Perugia, Italy, 1990. Clin Exp Hypertens 1991;B10:93.

Duvekot JJ, Cheriex EC, Pieters FAA, Menheere PPCA, Peeters LLH.

Altered hemodynamic/renal adaptation to early pregnancy: a potential contributor to the development of IUGR? Dutch Perinatal Biology Study Group, 1990.

Duvekot JJ, Cheriex EC, Pieters FAA, Peeters LLH.

The development of IUGR and/or preeclampsia is preceded by defective maternal cardiovascular adaptation in early pregnancy. In: Abstracts 39th Annual Scientific Meeting of the Society for Gynecologic Investigation, San Antonio, USA, 1992, No. 129.

Duvekot JJ, Cheriex EC, Pieters FAA, Menheere PPCA, Peeters LLH.

Nonpregnant hyperdynamic cardiac function predisposis to the development of intra-uterine growth retardation. 8th International Congress of the ISSHP, Buenos Aires, Argentine, 1992. Clin Exp Hypertens 1993;B12:93.

Duvekot JJ, Cheriex EC, Pieters FAA, Peeter LLH.

Early maternal hemodynamic adaptation to pregnancy is consistent with ongoing compensation of a state of hypovolemia. In: Abstracts 2nd World Congress of Perinatal Medicine, Rome, Italy, 1993.

Duvekot JJ, Cheriex EC, Pieters FAA, Menheere PPCA, Peeters LLH.

Impaired fetal growth is preceded by maladaptation in volume homeostasis in very early pregnancy. In: Abstracts 41st Annual Scientific Meeting of the Society for Gynecologic Investigation, Chicago, USA, 1994, No. 370. 


\section{CURRICULUM VITAE}

De auteur van dit proefschrift werd geboren op 7 juni 1958 in de Nederlands Hervormde pastorie te Zonnemaire (Zeeland). In 1976 behaalde hij het gymnasium-B diploma aan de Rijksscholengemeenschap te Brielle. Door de speling van het lot studeerde hij van 1976 tot 1977 scheikunde aan de Rijksuniversiteit Leiden. Van 1977 tot 1984 studeerde hij geneeskunde aan de Erasmus Universiteit te Rotterdam, alwaar hij in 1984 het artsexamen behaalde. Tijdens zijn studie was hij onder andere werkzaam als student-assistent op de afdelingen natuurkunde en gynaecologie/obstetrie. Op de afdeling gynaecologie/obstetrie werd onder meer onderzoek verricht naar de ontwikkeling van kunstmatig cervixslijm. In het kader hiervan werd in de zomer van 1982 een studiestage doorgebracht aan het instituut voor "Animal Science" van de Cornell Universiteit, Ithaka, U.S.A. (hoofd: Prof.dr. R.H. Foote). Van 1984 tot 1986 was hij werkzaam als arts-assistent gynaecologie in het Reinier de Graaf Gasthuis te Delft. Van 1986 tot 1987 was hij werkzaam als arts-assistent gynaecologie in de St. Elisabeth Kliniek (Vroedvrouwenschool) te Heerlen. Op 1 april 1987 werd een aanvang gemaakt met de opleiding tot gynaecoloog in het Academisch Ziekenhuis te Maastricht (opleider: Prof.dr. J. de Haan). Vanaf 1 juli 1991 vervolgde hij zijn opleiding op de afdeling gynaecologie van het De Wever-Ziekenhuis te Heerlen (opleider: Dr. J. Stoot). Sinds 1 april 1992 is hij werkzaam als gynaecoloog, met als aandachtsgebied obstetrie, in het St. Clara Ziekenhuis te Rotterdam.

Het in dit proefschrift beschreven wetenschappelijk onderzoek is vanaf 1989 verricht op de afdelingen gynaecologie, cardiologie, klinische chemie en nucleaire geneeskunde van het Academisch Ziekenhuis te Maastricht. 


\section{Publication of this thesis}

\section{was financially supported by:}

- Abbott B.V.

- Dr. W.S. Duvekot

- Entermed B.V.

- Ferring Nederland B.V.

- Hoechst Holland N.V.

- Organon Nederland B.V.

- Pfizer Nederland B.V.

- Dr. A.A. van Puyvelde Fonds

- Schering Nederland B.V.

- SmithKline Beecham Farma

- Sonotron B.V.

- Syntex B.V.

- Zeneca Farma 

